

\section{All rights reserved}

\section{INFORMATION TO ALLUSERS}

The quality of this reproduction is dependent upon the quality of the copy submitted.

In the unlikely event that the author did not send a complete manuscript and there are missing pages, these will be noted. Also, if material had to be removed, a note will indicate the deletion.

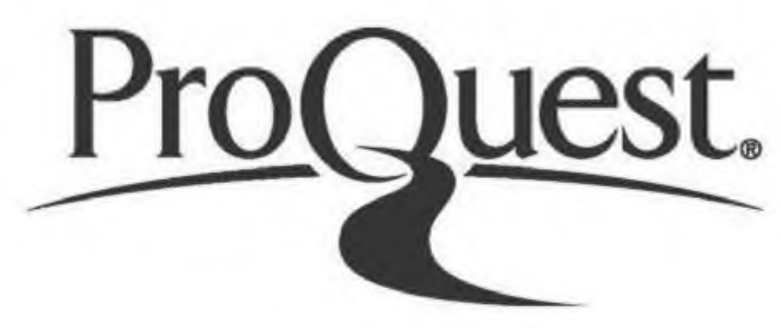

Published by ProQuest LLC (2017). Copyright of the Dissertation is held by the Author.

All rights reserved.

This work is protected against unauthorized copying under Title 17, United States Code Microform Edition (c) ProQuest LLC.

ProQuest LLC.

789 East Eisenhower Parkway

P.O. Box 1346

Ann Arbor, MI 48106-1346 


\title{
THE EFFECT OF ELECTRICAL CLAMPING FORCES ON THE MECHANICS OF PARTICULATE SOLIDS $/ 994$
}

\author{
A dissertation submitted towards \\ the Degree of Doctor of Philosophy \\ by \\ Catherine Michelle Martin \\ Department of Chemical and Process Engineering \\ University of Surrey
}

December 1994 


\section{SUMMARY}

The objective of this research is to advance the understanding of the effect of electrical clamping forces on the mechanics of particulate solids. These forces are produced when an electric field is applied to a densely-packed bed of particles by two electrodes which are in direct contact with the particles. Particle-electrode contacts and inter-particle contacts cause electric current to flow through the bed of particles. At the contact points, the current is constricted to flow through small regions of contact. Due to the high electrical resistance near the point of contact, the local electric field across the surfaces surrounding the contact area is greatly enhanced. Hence, the electrical clamping force is generated across the surfaces. These forces play a significant role in a number of industrial applications of electric fields. In particular, the Electromechanical Valve for Solids, which was developed at the University of Surrey for controlling the flow of granular materials, is considered in this work.

Using existing models for the electrical clamping force at a single contact, the interactions between the electrical and mechanical forces in bulk particle assemblies of cohesionless glass spheres are analysed. Wall and internal yield stresses are analysed by transforming the microscopic single contact electrical force into macroscopic representations of electrical stress. Using a continuum mechanics approach, the electrical stresses are superimposed on the mechanical stresses as additional external stresses. The important assumption here is that the yield locus remains unchanged with application of the electric field. This approach does not provide a satisfactory description of the bulk failure of the particulate solids as the electrical stresses are substantially overpredicted by theory as compared to the experimental results.

The single contact electrical clamping force models are also used to analyse the formation of arches under influence of an electric field. The investigation of arching is undertaken because halting of flow in the Electromechanical Valve for Solids occurs by the formation of stable arches over the electrode openings. The Mohr-Coulomb yield criterion requires the existence of cohesion to support the formation of stable arches. Therefore, it is speculated that the electrical clamping force may act as a cohesive force rather than an external compressive body force on the 
particle. Analysis of arching in a wedge-shaped hopper using both approaches is inconclusive.

A close look at the models of the electrical clamping force reveals that the discrepancy between the theoretical predictions and experimental results may be due to the assumption in the models of Hertzian contact deformation in response to the electric field. In view of the difficulties with the existing models, it is decided to make a direct evaluation of the electrical clamping force experimentally.

The electrical clamping forces at single contacts have been measured here for the first time. These measurements required the development of a technique which uses a modified nanoindentation device capable of measuring very small forces. The detachment forces between glass spheres and a polished stainless steel plate with an electric field have been measured. The experimental results are one to two orders of magnitude smaller than predicted by the models, corroborating the macroscopic yield stress results. It is concluded that the single contact models do not satisfactorily describe the contact deformation under an electric field and hence are unreliable. Attempts are made to quantify the contact area by inference from measurements of electrical resistance using the Sphere Resistance theory. However, this method is highly sensitive to the value of surface resistivity, which cannot be measured accurately, and is therefore unsuitable for evaluation of the contact area. 
(C) C M Martin 1994 


\section{ACKNOWLEDGEMENTS}

The work described in this dissertation was carried out in the Department of Chemical and Process Engineering at the University of Surrey. It is my own work and includes nothing that is the result of collaboration except where specifically acknowledged in the text.

There are a number of people who contributed technically and philosophically to this research. Most significantly, I must thank $\mathrm{D} r \mathrm{M}$ Ghadiri for his supervision throughout this project. The research grew from his initial concept and this dissertation reflects his influence in many ways. Thanks also go to my other two supervisors, Professor $\mathrm{R}$ Clift and $\mathrm{Dr} U$ Tüzün, for their creative and stimulating ideas. For his ongoing helpfulness, I thank $\mathrm{Mr} P$ Arteaga, especially for training me to use the Nanotest. I also thank Dr J Morgan for her work on the EVS at a very early stage in this project, and $\mathrm{Dr} B$ Formisani for his contributions to the shear cell work and for his continued interest in this research. Finally, I am grateful to several people in the electrical and mechanical workshops who turned my drawings into working equipment. These include $\mathrm{Mr}$ D Arnall, Mr C Mann, Mr S Pool, Mr G Shurlock and Mr P Pennington.

Finally, my research was made possible by financial support from the Science and Engineering Research Council and from the Research Committee and Technology Transfer Committee of the University of Surrey. I am thankful to these organizations for their support.

C M Martin

December 1994 
1.1 Background

1.2 The Electromechanical Valve for Solids

1.3 Outline and scope

CHAPTER 2 REVIEW OF PREVIOUS INVESTIGATIONS OF THE ELECTRICAL CLAMPING FORCE
2.1 Introduction
2.2 Review of previous work
2.3 Conclusions

CHAPTER 3 OBSERVATIONS OF MACROSCOPIC BULK BEHAVIOUR OF PARTICULATE SOLIDS WITH ELECTRIC FIELDS
3.1 Introduction
3.2 Internal and wall shear stresses at failure
3.3 Arching in hoppers
3.4 Steady-state flow
3.5 Dynamic response to changing fields
3.6 Conclusions

CHAPTER 4 ANALYSIS OF MACROSCOPIC BULK BEHAVIOUR OF PARTICULATE SOLIDS WITH ELECTRIC FIELDS
4.1 Introduction
4.2 Transformation of microscopic force models to macroscopic representation
4.3 Internal and wall shear stresses
4.4 Arching in hoppers
4.5 Steady-state flow
4.6 Conclusions ELECTRICAL CLAMPING FORCE

5.1 Introduction

5.2 Experimental method

5.3 Results of single contact detachment experiments

5.4 Analysis of detachment force

5.5 Analysis of macroscopic behaviour using single contact force measurements

5.6 Discussion on fracture mechanics approach to

electrical clamping force

5.7 Analysis of contact resistance

5.8 Conclusions 
CHAPTER 6 CONCLUSIONS

CHAPTER 7 RECOMMENDATIONS FOR FUTURE WORK

REFERENCES

APPENDIX 4.1 REVIEW OF ELECTRICAL CLAMPING FORCE MODELS FOR SPHERES

APPENDIX 4.2 ARCH MODELLING

TABLES

162

FIGURES 


\section{NOMENCLATURE}

\section{Roman}

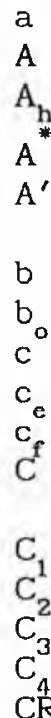

d

$d_{0}$

$d^{p}$

$d_{1}^{w}, d_{2}, d_{3}, d_{4}$

D.

$\mathrm{D}_{\mathrm{h}}^{*}$

$\mathrm{D}^{\mathrm{h}}$

DC̊F

$E_{a}$

$E_{\text {eff }}$

$E_{\text {lim }}$

$\mathrm{E}_{\mathrm{m}}$

$E_{\text {max }}$

$\frac{f_{c}^{c}}{f_{e}}$

$f_{n}^{m}$

$f_{p}$
$f_{t}^{t}$
$F$
: contact cap radius for sphere

area of electrode

surface area of hemisphere

effective area of orifice of general shape

circular perimeter around sphere through

which current flows

: $y$-intercept of line in equation (4.38)

: orifice width

: cohesion/adhesion without electric field

electrical cohesion/adhesion

coefficient of flow

: function used in arching models defined in Appendix 4.2

: parameter in equation (5.50)

: parameter in equation (5.51)

: parameter in equation (5.53)

: parameter in equation (5.54)

: calibration factor between LVDT output and capacitance bridge rectified output

contact diameter between two cylinders

internal side length of square mesh opening

particle diameter

wire diameter

diameter of electrodes in Fig. 5.22

diameter of cylinder

diameter of circular grid

hydraulic diameter of orifice

diameter of orifice in hopper

: calibration factor between displacement and capacitance bridge output in Nanotest

: average applied electric field

$:\left(\frac{1-v_{r 1}^{2}}{\pi E_{m 1}}+\frac{1-v_{r 2}^{2}}{\pi E_{m 2}}\right)^{-1}$

: limiting value of electric field in contact gap at $\theta$

: Elastic modulus; $\stackrel{\circ}{\mathrm{E}}_{\mathrm{m} 1}, \mathrm{E}_{\mathrm{m} 2}$ : Elastic moduli of two contacting bodies

electric field breakdown strength

electric field in gap outside contact

in direction normal to contact

: unconfined yield stress

: electrical clamping force

: average electrical clamping force in direction of average electric field

mechanical normal force on contact

normal force applied on specimen in

Nanotest (compressive or tensile)

: polarization force

: total normal force on contact

normal force on contact

detachment force for sphere/wall

contact with electric field $\mathrm{m}^{2}$

$\mathrm{m}^{2}$

$\mathrm{m}^{2}$

$\mathrm{m}$

$\mathrm{N} \mathrm{m}^{-2}$

$\mathrm{m}$

$\mathrm{N} \mathrm{m}^{-2}$

$\mathrm{N} \mathrm{m}^{-2}$

N V ${ }^{-\gamma} \mathrm{m}^{\gamma-2}$

$\mathrm{N} \mathrm{m} \mathrm{v}{ }^{-1}$

$\mathrm{J}(\mathrm{V} \mathrm{m})^{-1}$

$(\mathrm{m} / \mathrm{V})^{1 / 3}$

$(\mathrm{m} / \mathrm{V})^{1 / 3}$

$\mathrm{V} \mathrm{V}^{-1}$

m

$\mathrm{m}$

$\mathrm{m}$

$\mathrm{m}$

m

m

$\mathrm{m}$

m

$\mathrm{m}$

$\mathrm{m} \mathrm{V}^{-1}$

$\mathrm{V} \mathrm{m}{ }^{-1}$

$\mathrm{N} \mathrm{m}^{-2}$

$\mathrm{V} \mathrm{m}^{-1}$

$\mathrm{N} \mathrm{m}{ }^{-2}$

$\mathrm{V} \mathrm{m}^{-1}$

$\mathrm{V} \mathrm{m}^{-1}$

$\mathrm{N} \mathrm{m^{-2 }}$

$\mathrm{N}$

$\mathrm{N}$

$\mathrm{N}$

$\mathrm{N}$

$\mathrm{N}$

$\mathrm{N}$

N

N 


\begin{tabular}{|c|c|c|c|}
\hline $\mathrm{F}_{\mathrm{es}}$ & : & electrostatic attractive force & $\mathbf{N}$ \\
\hline $\mathrm{F}_{\mathrm{m}}^{\mathrm{es}}$ & : & $\begin{array}{l}\text { magnetic force between copper coil } \\
\text { and magnet in Nanotest device }\end{array}$ & $\mathrm{N}$ \\
\hline $\mathrm{F}_{\mathrm{s}}$ & : & $\begin{array}{l}\text { detachment force between two spheres (or } \\
\text { sphere and wall) without electric field }\end{array}$ & $\mathrm{N}$ \\
\hline$F_{t}$ & : & tensile force & $\mathrm{N}$ \\
\hline$g^{2}$ & : & gravitational acceleration & m $s^{-2}$ \\
\hline G & : & gap between electrodes in Fig. 5.22 & m \\
\hline h & : & $\begin{array}{l}\text { distance between surfaces in annular crack } \\
\text { in cylinder }\end{array}$ & $\mathrm{m}$ \\
\hline $\mathrm{H}$ & : & $\begin{array}{l}\text { distance from virtual apex of top surface } \\
\text { of material in hopper }\end{array}$ & $\mathrm{m}$ \\
\hline I & : & electric current & A \\
\hline IEG & : & inter-electrode gap & m \\
\hline k & : & particle shape parameter in equation (4.47) & - \\
\hline $\mathrm{k}_{\mathrm{c}}, \mathrm{k}_{\mathrm{c1}}, \mathrm{k}_{\mathrm{c} 2}$ & : & $\begin{array}{l}\text { parameter which is function of } \nu_{r} \text { and } E_{m} \\
\text { of contacting bodies, defined in } \\
\text { equation }(5.46)\end{array}$ & $\mathrm{m}^{2} \mathrm{~N}^{-1}$ \\
\hline K & : & parameter in equation (5.20) & $\mathrm{N}(\mathrm{V} \mathrm{m})^{-1}$ \\
\hline $\mathrm{K}^{\prime}$ & : & parameter in equation (5.15) & $N(V, m)^{-1}$ \\
\hline $\mathrm{K}_{\mathrm{c}}$ & : & $\begin{array}{l}\text { parameter which is function of } \nu_{r} \text { and } E_{m} \\
\text { of contacting bodies, defined in } \\
\text { equation }(5.46)\end{array}$ & $N m^{-2}$ \\
\hline L & : & distance between electrodes & $\mathrm{m}$ \\
\hline LCF & : & $\begin{array}{l}\text { calibration factor between applied force } \\
\text { and coil voltage in Nanotest }\end{array}$ & $\mathrm{N} \mathrm{V}^{-1}$ \\
\hline $\mathrm{m}$ & : & slope of line in equation ( 4.38 ) & $\mathrm{N}(\mathrm{V} \mathrm{m})^{-1}$ \\
\hline$m_{w}$ & : & $\begin{array}{l}\text { mass of dead weights used to calibrate } \\
\text { applied force in Nanotest }\end{array}$ & $g$ \\
\hline $\mathrm{M}_{\mathrm{w}}$ & : & $\begin{array}{l}\text { weight of dead weights used for calibration } \\
\text { in Nanotest }\end{array}$ & $\mathrm{N}$ \\
\hline n & : & $\begin{array}{l}\text { total equivalent number of mesh openings } \\
\text { in circular grid }\end{array}$ & - \\
\hline $\mathrm{N}$ & : & $\begin{array}{l}\text { coordination number, } \\
\text { i.e. number of contacts per particle }\end{array}$ & - \\
\hline $\mathrm{P}$ & : & average perimeter of electrodes in Fig. 5.22 & m \\
\hline $\mathrm{P}$ & : & interstitial pressure & $\mathrm{N} \mathrm{m}^{-2}$ \\
\hline$P_{t}$ & : & mean principal stress in Fig. 4.3 & $\mathrm{~N} \mathrm{~m}^{-2}$ \\
\hline$P_{1}^{t}, P_{2}$ & : & stresses defined in Fig. A4.4 & $\mathrm{N} \mathrm{m}{ }^{-2}$ \\
\hline & : & distance from virtual apex in hopper & $\mathrm{m}$ \\
\hline$r_{\mathrm{c}}$ & : & radial distance from centre of cylinder & $\mathrm{m}$ \\
\hline$r_{0}^{c}$ & : & distance from virtual apex at orifice & $\mathrm{m}$ \\
\hline $\mathrm{R}^{\circ}$ & : & radius of sphere & $\mathrm{m}$ \\
\hline$R_{1}, R_{2}$ & : & $\begin{array}{l}\text { radii of two contacting spheres, } \\
\mathrm{R}_{2}=\infty \text { for wall }\end{array}$ & $\mathrm{m}$ \\
\hline$R_{a}$ & : & $\begin{array}{l}\text { resistance measured between annular } \\
\text { electrodes in Fig. } 5.22\end{array}$ & $\Omega$ \\
\hline$R_{b}$ & : & bulk resistance & $\Omega$ \\
\hline$R_{c}^{0}$ & : & $\begin{array}{l}\text { parameter which is function of contacting } \\
\text { radii defined in equation }(5.45)\end{array}$ & $\mathrm{m}$ \\
\hline$R_{\text {eff }}$ & : & $=\frac{1}{2}\left(\frac{1}{R_{1}}+\frac{1}{R_{2}}\right)$ & $\mathrm{m}^{-1}$ \\
\hline$R_{\text {el }}$ & : & internal resistance of electrometer & \\
\hline $\mathrm{R}_{\mathrm{m}}^{\mathrm{el}}$ & : & radius of Mohr's circle in Fig. A4.3 and A.4.4 & $\mathrm{N} \mathrm{m}{ }^{-2}$ \\
\hline $\mathrm{R}_{\mathrm{s}}$ & : & resistance of sphere & $\Omega$ \\
\hline$R_{t}$ & & radius of Mohr's circle in Fig. 4.3 & $\mathrm{~N} \mathrm{~m}^{-2}$ \\
\hline $\mathrm{R}_{\theta}$ & : & resistance of sphere over angle $\theta$ & $\Omega$ \\
\hline
\end{tabular}


: yield shear stress under normal stress $\mathrm{w}_{1}$

$\mathrm{N} \mathrm{m}^{-2}$ in Fig. 4.3

$-s_{1}$ : yield shear stress acting in direction at $90^{\circ}$ to $\mathrm{s}_{1}$

: average distance between particle centres at wall

$\mathrm{U}^{\mathrm{e}}$
$\mathrm{U}^{\mathrm{p}}$
$\mathrm{U}^{\mathrm{P}}$
$\mathrm{U}^{\mathrm{s}}$
$\mathrm{V}$

: stored elastic energy

: work done by applied load

: surface energy

: total energy

applied voltage

: voltage applied to coil in Nanotest

: dynamic holding voltage

: voltage potential across surfaces

in annular crack in cylinder

VR

: static holding voltage

: voltage output response factor for LVDT

$\mathrm{w}_{1}$

: applied normal mechanical stress in Fig. 4.3

: mechanical stress acting in direction at $90^{\circ}$ to $\mathrm{w}_{1}$

W

W

$\mathrm{W}^{\mathrm{c}}$

$W_{0}^{m}$

mass flowrate

: total flowrate through circular grid

mass flowrate through one mesh opening

: mass flowrate without an applied electric field

WV

: calibration factor between mass of dead weights and coil voltage

$\mathrm{x}$

$\mathrm{X}$

: length in Nanotest device defined in Fig. 5.3

: function used in arching models defined in Appendix 4.2

$\mathrm{X}_{\mathrm{t}}$

length on Mohr's circle defined in Fig. 4.3

length in Nanotest device defined in Fig. 5.3

length in Nanotest device defined in Fig. 5.3

function used in arching models defined in Appendix 4.2

$Y_{t}$

length on Mohr's circle defined in Fig. 4.3

distance from surface of sphere to plane of contact, normal to contact

Z

function used in arching models defined in Appendix 4.2

: function used in arching models defined in Appendix 4.2

: critical value of $\mathrm{Z}$ for arching defined in Appendix 4.2

m

$$
\text { J }
$$

$\mathrm{J}$

$\mathrm{J}$

$\mathrm{J}$

V

V

V

V

V

$\mathrm{m} \mathrm{V} \mathrm{V}^{-1}$

$\mathrm{N} \mathrm{m}^{-2}$

$\mathrm{N} \mathrm{m}^{-2}$

$\operatorname{kg} \mathrm{s}^{-1}$

$\mathrm{kg} \mathrm{s} \mathrm{s}^{-1}$

$\mathrm{kg} \mathrm{s} \mathrm{s}^{-1}$

$\mathrm{kg} \mathrm{s}$

$\mathrm{g} \mathrm{V}^{-1}$

$\mathrm{m}$

$-$

$\mathrm{N} \mathrm{m}{ }^{-2}$

$\mathrm{m}$

m

$-$

$\mathrm{N} \mathrm{m}^{-2}$

$\mathrm{m}$

$\mathrm{N} \mathrm{m}^{-1}$

$\mathrm{N} \mathrm{m}^{-2}$

$\mathrm{N} \mathrm{m}^{-1}$

\section{Greek}

$\alpha$

$\beta$

$\gamma$

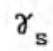

$\Gamma$

: lumped parameter describing particle and interstitial fluid properties in electrical clamping force models

: angle between plane of major principal stress and wall; $\beta_{a}$ : active stress state, $\beta_{\mathrm{p}}$ : passive stress state

: power index in electrical clamping force models

: surface energy per unit area of contacting surfaces

$:=\mathrm{g} \cdot \rho_{\mathrm{d}}$

$\mathrm{N} \mathrm{V} \mathrm{m}^{\gamma}$

rad, deg

$\mathrm{J} \mathrm{m}^{-2}$

$\mathrm{kg}(\mathrm{m} \mathrm{s})^{-2}$ 
$\Delta \mathrm{r}$

$\Delta \mathrm{W}$

$\varepsilon$

$\varepsilon_{0}$

$\varepsilon_{f}$

$\varepsilon_{\mathrm{p}}$

$\zeta^{s}$

n

$\theta$

$\theta_{c}$

$\theta^{\circ}$

$\lambda$

$\mu$

$\nu$

$v_{\mathrm{r}}$

$\xi$

$\rho_{\mathrm{b}}$

$\rho_{\mathrm{d}}$

$\rho_{\mathrm{f}}$

$\rho_{\mathrm{s}}$

$\sigma$

$\sigma_{c}$

$\sigma_{\mathrm{e}}$

$\sigma$

e1, min'

$\sigma_{\mathrm{e} 1, \max }$

$\sigma_{\mathrm{e} 2}$

$\sigma_{m}$
$\sigma_{p}$
$\sigma_{t}$
$\sigma_{1}$
$\sigma_{2}$
$\tau$
$v$
$\phi$
$\chi$
: angle of internal friction

deformation normal to contact

vertical component of electrical

lift force per unit depth from walls

: vertical component of mechanical

lift force per unit depth from walls

: net downward force per unit depth from material surrounding layer

effective length of current path over sphere

height of arched element

weight of arched layer per unit depth

voidage

absolute permittivity of free space

absolute permittivity of interstitial fluid

absolute permittivity of particle

bulk relative permittivity of solids

parameter in equation (4.2)

parameter in equation (4.2)

angle over sphere

critical angle over sphere where local

electric field reaches breakdown strength

contact angle for sphere

hopper half angle

parameter in flow model equation (4.57)

coefficient of friction

angle defined in equation (4.20);

$v_{a}$ : active stress state,

$v_{\mathrm{p}}^{\mathrm{a}}$ : passive stress state

: Poisson's ratio; $\nu_{r 1}, \nu_{r 2}$ : Poisson's ratios

for two contacting bodies

parameter in flow model equation (4.57)

bulk volume resistivity of packed bed

bulk density of granular material

flowing density of granular material

surface resistivity

mean principal stress

attractive electrical stress between

surfaces of axially contacting cylinders

: mechanical stress required to break contact

between cylinders without electric field

electrical clamping stress

major principal electrical stress

low and high values of $\sigma_{\text {el }}$ which produce

tangency of combined stress Mohr's circle

with yield locus in Fig. 4.3

minor principal electrical stress

fracture stress for annular crack in

cylinders with electric field

normal mechanical stress

stress due to polarization forces

total normal stress

major principal stress

minor principal stress

yield shear stress

parameter defined in equation (4.9)

angle of wall friction

angle of rotation of Mohr's circle

in Fig. 4.3 rad, deg

m

$\mathrm{N} \mathrm{m}{ }^{-1}$

$\mathrm{N} \mathrm{m}^{-1}$

$\mathrm{N} \mathrm{m}{ }^{-1}$

$\mathrm{m}$

$\mathrm{m}$

$\mathrm{N} \mathrm{m}^{-1}$

-

$\mathrm{F} \mathrm{m}^{-1}$

$F \mathrm{~m}^{-1}$

$\mathrm{F} \mathrm{m}{ }^{-1}$

-

-

rad, deg

rad, deg

rad, deg

rad, deg

$\mathrm{m}^{\xi} \mathrm{kV}^{-\xi}$

rad, deg

$\Omega \mathrm{m}$

$\mathrm{kg} \mathrm{m} \mathrm{m}^{-3}$

$\mathrm{kg} \mathrm{m}^{-3}$

$\Omega \mathrm{mm}^{-1}$

$\mathrm{N} \mathrm{m}^{-2}$

$\mathrm{N} \mathrm{m}{ }^{-2}$

$\mathrm{N} \mathrm{m}^{-2}$

$\mathrm{N} \mathrm{m}^{-2}$

$\mathrm{N} \mathrm{m}^{-2}$

$\mathrm{N} \mathrm{m}^{-2}$

$\mathrm{N} \mathrm{m}{ }^{-2}$

$\mathrm{N} \mathrm{m}^{-2}$

$\mathrm{N} \mathrm{m}^{-2}$

$\mathrm{N} \mathrm{m}^{-2}$

$\mathrm{N} \mathrm{m}^{-2}$

$\mathrm{N} \mathrm{m}^{-2}$

$\mathrm{N} \mathrm{m}^{-2}$

$\mathrm{N} \mathrm{m^{-2 }}$

rad, deg

rad, deg 
angle between normal to contact and

rad, deg direction of average electric field

rad, deg 


\section{CHAPTER 1}

\section{INTRODUCTION}

\subsection{BACKGROUND}

There are many industrial and laboratory applications where particles experience the effect of electrical forces. These forces can occur as a side effect of a non-electric process or can be deliberately induced to produce a desired behaviour. The former case includes frictional charging, or triboelectrification, of insulating particles in flow in pipes and gas fluidized beds. Charging of particles occurs by charge transfer from particle-wall impacts. This can produce undesirable effects which include coating of surfaces with particles and erratic movement of particles, as well as the safety hazard of discharge of accumulated electric charge. Due to its common occurrence in industry, especially in pneumatic conveying of powders and granular materials, this phenomenon has attracted some research interest [1-8]. However such behaviour is difficult to quantify and predict, and can generally be avoided in industry by taking suitable actions such as humidification to increase the surface conductivity of the particles and earthing of pipework.

In the case where electrical forces are deliberately induced, electric fields are applied to particles which are either charged or become polarized to control their movement to a desired surface. Examples of these electrical processes include electrostatic precipitation $[9,10]$ and electrically enhanced filtration [11-13] for removing particulates from gases, and dielectrophoresis [14] for analysing mixtures of organic materials, such as cells. In electrostatic precipitators, charged particles in an electric field migrate through the gas to be collected onto the passive electrode. In electrically enhanced filtration, electric fields are applied to charged or uncharged particles to enhance collection efficiency. In dielectrophoresis, uncharged particles become polarised in nonuniform electric fields and move in the direction of highest field gradient. In these cases, particles are dispersely distributed in an insulating fluid medium so that there is no continuous interparticle contact and thus, no electric current flowing between the particles. The movement of the particles is due to the forces generated on the charged or polarized particles by the electric field. 
The behaviour of particles in electric fields is also affected when they are in continuous contact, i.e. in a densely packed bed. This situation applies to the cake formed in electrostatic precipitators and to electrofluidised beds. These two processes have recently been the subject of similar research investigations where the fundamental mechanism for generation of the electrical forces was examined.

In electrostatic precipitators, there is strong adhesion of the deposited dust layer to the passive electrode against the pulling force from the active electrode and the gravitational force. The strength of the cake adhesion cannot be explained simply by the electrostatic force of attraction between two electrodes filled with dielectric material $[15,16]$.

Electrofluidised beds with gas-solid phases have been investigated for a number of reasons, e.g. to produce ohmic heating, for the filtration of small particulates and aerosols, to improve gas-particle contact by controlling bubble size [17-19]. In electrofluidised beds, electric fields are found to modify the bed dynamics. Large pressure drop overshoots are measured at the point of fluidisation, and in the fluidised state strings of particles are observed to form between the electrodes. Neither the electrostatic force between the electrodes nor polarization forces on the particles by the electric field are large enough to account for the behaviour in electrofluidised beds $[19,20]$.

The clue to explain the relevant mechanism in both the above cases was the observation that the bed behaviour depended on the particles being in contact such that there was a conductive path through the bed. Hence, it was concluded that the dominating mechanism for the forces between the particles was the flow of current through the bed $[15,16,18-20]$. In their research on electrostatic precipitators, Moslehi and Self termed this force the electrical clamping force [16]. These forces are due to the constriction of electric current at the contact points, where the local electric field across the gap surrounding the contact area is greatly enhanced. Provided that the particles are not too conductive so as to draw excessively high currents above the power limit of the high voltage source, nor too resistive so as to draw such low currents that there is insufficient effect, the electrical clamping forces may be sufficiently large and comparable in magnitude with external forces, such as those due to gravity, so that the bulk failure characteristics of the solids are 
substantially altered.

Other areas where the electrical clamping forces may be playing a significant role include electrostatically enhanced fabric filtration and electrorheological fluids. In both these fields, the influence of the electrical clamping force appears to be largely ignored. In electrostatically enhanced fabric filtration, dust particles are artificially charged and/or an electric field is applied to the filter in order to produce a more porous cake structure and thus a lower pressure drop. Only recently has it been suggested that the electrical clamping forces resulting from charge leakage through the cake produce a stronger cake which has less tendency to collapse and thus a more porous packing is maintained [11].

Extensive research has been carried out in the field of electrorheological fluids, although none has evaluated the contribution of the electrical clamping force [21,22]. An electrorheological (ER) fluid is a suspension of dielectric particles in a non-conducting fluid. On application of electric fields in excess of about $1 \mathrm{kV} \mathrm{mm}$, the shear stress of an ER fluid can be increased by orders of magnitude to a point where the fluid is essentially 'frozen'. The rheological behaviour of an ER fluid is generally interpreted as Bingham plastic-like with a static yield stress. Increased yield stresses in excess of $1 \mathrm{kPa}$ can be transmitted and switched in the order of milliseconds.

The particles used in ER fluids are typically 1 to $100 \mu \mathrm{m}$ in diameter and the volume fraction of particles is generally between 0.1 and 0.4 . A vast range of combinations of substances have been used to form ER fluids, including silica, starch, and cellulose for the particulate phase and silicone oil, chlorohydrocarbons and corn oil for the liquid phase. Until recently, it was thought that ER fluids must also contain a polar fluid as an additive, such as water or a surfactant, but recently 'dry' ER fluids have been developed, thus disproving one theory that liquid bridges were responsible for the greatly enhanced shear stress of ER fluids.

The first ER fluids were developed by Winslow [23] who recognized their potential for use in controlling devices such as clutches, brakes and valves. Recently, there has been renewed interest in ER fluid applications. However, much of the research has been aimed at optimising 
the design of ER systems for specific applications rather than developing an understanding of the fundamental physics involved in ER fluid behaviour. Therefore, there is still a great deal of uncertainty about the exact mechanisms responsible for ER fluids. Most theories and models assume that the ER phenomenon is due to polarization of the particles in the electric field and the resulting coulombic interactions between particles. This explanation ignores the effects of interparticle contact. Yet, experimental evidence shows that when there is no flow, i.e. the applied shear stress is less than the static yield stress, the particles form chains which extend from one electrode to the other. In fact, these chains are considered to be the source of the resistance to flow which results in the static yield stress of an ER fluid.

All ER fluids are also found to show some conductance. A few sources of the electric current have been proposed, such as bulk conduction due to a small amount of water dissolving in the liquid phase but this is not considered to be the dominant source of loss. It is more likely, that conduction occurs through the particle chains via the interparticle contacts. Supporting evidence of this conduction process is the observation that at particle volume fractions greater than about 0.4 , the electrodes short out. Therefore, at shear stresses less than the static yield stress when the ER fluid is not flowing, it seems likely that electrical clamping forces are generated at the contacts between the particles. However, the contribution of the electrical clamping forces has so far been ignored in theoretical models of the static yield stress $[21,22]$.

Recently, another useful application of the electrical clamping forces has appeared in the form of the Electromechanical Valve for Solids 124,25]. In the Electromechanical Valve for Solids, or EVS, a DC electric field is applied to a densely packed bed of particles via two electrodes inserted in the bed. In this way, the EVS is used to provide on/off operation and continuous flow control of granular materials. As for the other electrical processes described above, the operation of the EVS depends on the particles maintaining continuous contact and being able to conduct a small current. Therefore, the dominating electrical mechanism affecting the performance of the EVS is the electrical clamping force.

In order to optimise the design and operation of the EVS, a quantitative 
model for its performance is required. The research documented in this dissertation was initiated based on the ultimate goal of developing a model for flow in the EVS. In order to achieve this final result, the overall objective of the research was to develop a much greater understanding, qualitative and quantitative, of the effect of the electrical clamping forces on the flow of granular materials. In order to provide an understanding of the scope and design of the research, a detailed description of the EVS is given below, followed by an overview of the structure of this dissertation.

\subsection{THE ELECTROMECHANICAL VALVE FOR SOLIDS}

In many industrial processes involving granular materials, regulation and control of the flow of material between process units is extremely important for efficient plant operation and final product quality. The Electromechanical Valve for Solids provides several advantages over the mechanical devices which are traditionally used in industry for flow control of granular materials, such as screw conveyors and rotary feeders. These devices have serious drawbacks which stem from the inherent features of their design and operation. Rotation of the moving component against the stationary casing causes attrition when handling delicate particles, and erosion or seizure when handling hard and abrasive materials. The devices are also slow in response and have short-term fluctuating characteristics and long-term drift. Because it has no moving parts, the Electromechanical Valve for Solids does not suffer from the extreme attrition and wear problems of mechanical valves, and it has a very fast response time $[26]$.

The Electromechanical Valve for Solids is not the only non-mechanical concept developed for flow control of particulate solids. $\mathrm{L}, \mathrm{J}$ and $\mathrm{V}$ valves control flow with injected gas [27-29]. These valves are cheap and simple, but they do not have the fast reponse time of the Electromechanical Valve for Solids, especially when responding to a decrease in the flowrate of the injected gas. The Magnetic Valve for Solids has also been developed as a non-mechanical valve for controlling the flow of particulate solids [30]. The Magnetic Valve relies on the interparticle forces which are produced when a magnetizable powder is in a magnetic field. These forces lead to the formation of particle chains which bridge the gap between the 
electrodes and hence impede the flow of solids. Since relatively few materials are strongly magnetic, it is necessary to add a small quantity of magnetic particles to the bulk material for control with the Magnetic Valve. Doping in this manner is often unacceptable for the process. The magnetic properties of materials are also adversely affected by increased temperature. The Electromechanical Valve for Solids does not suffer from these restrictions and may be used with a wide range of materials under adverse conditions such as elevated pressures and temperatures.

The Electromechanical Valve for Solids (EVS) consists essentially of two electrodes which are installed within the duct in the path of the flowing material. The electrodes are connected externally to a high voltage supply (EHT) unit for establishing an electric field. For the electric field to be effective, a suitable electrode design must be used. Typical electrode configurations are shown in Fig. 1.1 for cylindrical and rectangular ducts. The upstream electrode may be in the form of a number of wires stretched across the duct or may simply consist of pins protruding into the duct. The downstream electrode may conveniently be in the form of a wire mesh or parallel wires fixed across the duct.

As discussed above, for the EVS to operate at all, it is essential that the flow in the space between the electrodes is in dense-phase form so that continuous interparticle and particle-electrode contacts are maintained. Observations of particle motion in the space between the electrodes indicate that as soon as the flow in this region becomes lean, the mechanism on which the EVS relies is lost completely. Therefore, the upstream electrode must not significantly obstruct the flow. A slide valve or flap door is also needed downstream of the electrodes, so that it can be closed during the initial filling of the column to obtain the material in a dense-phase state. Thereafter, the flap door is left open as the bed of material can be supported by the electric field. When a sufficiently high electric field is applied across the electrodes, flow of the material is completely halted. In this state, the material forms stable arches over the mesh openings of the downstream electrode. If a continuous DC field is applied, a steady-state flowrate is attained which is a function of the applied field. Alternatively, the electric field may be pulsed, in which case the frequency, pulse width and amplitude may all be varied to control the flow. A wide range of free-flowing granular materials have been tested successfully in the EVS, including sand, glass ballotini, coal, NaCl salt, 
and seeds [25].

These basic observations of flow behaviour in the EVS provide evidence for the fundamental mechanisms which are responsible for its operation. At the microscopic level, the fundamental mechanism for the interparticle electrical forces is the electrical clamping force. At the macroscopic level, the physical processes by which the local electrical forces manifest themselves as bulk phenomena, e.g. arching, must be investigated.

There are several theoretical models for the electrical clamping force $[15,16,19,20]$. These models depend on the mechanical properties of the contact points and on the limit of electric field breakdown in the interparticle gap. Most of the models relate the electrical clamping force to the average applied electric field strength, $E_{a}$, for a single contact between two spheres, or between a sphere and a conductive plane. Recently, an additional model was developed for the axial contact between two cylinders [31]. As single contact force measurements are notoriously difficult to make, there has so far been no attempt to measure this force directly. The verification of the electrical clamping force models has been inferred from measurements of the bulk behaviour of granular materials, e.g. pressure overshoot at incipient fluidization in electrofluidized beds, detachment stress between an electrode and a dust cake [15,19]. These analyses are few in number and are unsatisfactory. In order to model the flow behaviour in the EVS, more rigorous analyses of the macroscopic and microscopic representations of the electrical clamping forces were necessary. The results of these investigations are presented in this dissertation.

\subsection{OUTLINE AND SCOPE}

The main part of the dissertation commences in Chapter 2 with a review of previous investigations of the electrical clamping force. The approaches used in this previous work and the main experimental observations are reviewed briefly in order to establish the current level of knowledge on the electrical clamping forces.

Chapter 3 deals with the experimental evaluation of the flow behaviour of packed beds in electric fields. Three aspects of flow are considered: 
initiation and halting of flow, steady-state flow, and transient flow.

The initiation and halting of flow was investigated using two simple electrode geometries. In one experiment, the effect of electric fields on the internal failure of glass ballotini was examined. Yield stresses were measured for the material in a box-type shear cell where the electrodes were attached to the bottom of the box and under the lid. The case for wall shear stresses with electric fields had already been investigated experimentally by Robinson and Jones [32]. Their experimental results are presented in detail in Chapter 3 for comparison with the case of internal failure.

In the second experiment on halting and initiation of flow, the formation of stable arches by application of an electric field was investigated. An electrode geometry was used which consisted of a two-dimensional wedge-shaped hopper with electrodes along the side walls. This electrode system was used to measure the voltages required to prevent material from flowing and thus form a stable surface at the orifice of the hopper. An investigation of arching was undertaken because observations of particle movement in the EVS show that halting of the flow occurs by the formation of stable arches over the mesh openings of the downstream electrode. As this is observed for cohesionless materials, the arches must be supported by the electric field. Similarly to the shear cell experiments, the arching experiments were carried out using glass ballotini with a narrow size distribution $(600-710 \mu \mathrm{m})$. A spherical, cohesionless material was used with the electrode systems in the shear cell and arching experiments to provide simple systems for focusing on the role of the electrical clamping force.

The effect of electric fields on steady-state flow is described in Chapter 3 for flow in the EVS with electrode configurations as shown in Fig. 1.1. The effect of electric field strength and distribution is examined for flow in cylindrical columns of 64 and $115 \mathrm{~mm}$ I.D. and in a $200 \times 12 \mathrm{~mm}$ rectangular duct. Several materials were used in these tests, including sand, coal, $\mathrm{NaCl}$ salt, and glass ballotini.

The third aspect of flow which is considered in Chapter 3 is transient flow in response to changing electric fields. As with the work on steady-state flow, the investigation of transient flow considers flow in the EVS for a 
variety of granular materials. Flow behaviour with pulsating fields is described. Attempts to characterize the time constant of the EVS are discussed, and the response to Proportional-Integral-Differential control is evaluated. The measurement of the dynamic response of particle movement to changing electric fields is shown to be difficult because of the slow response of the measuring devices with respect to the response time of the particles themselves. Therefore, transient flow behaviour is not analysed further in this dissertation and remains to be examined in greater detail in future.

In the experimental work presented in Chapter 3, the surface resistivity of the materials is an important property. Except for metal powders, most particulate solids used in industry fall into the resistivity range $10^{5}-10^{11} \Omega \mathrm{m}$, i.e. semi-conducting to semi-insulating. This is the range of resistivities which can be used effectively in the EVS. Materials were chosen and the experimental conditions were controlled in the experiments in Chapter 3 to ensure sufficient surface conductivity such that the electrical clamping forces had a significant effect on the flow behaviour of the material, and the effects of tribocharging were negligible. In the case of the glass ballotini, the surfaces were coated with a surfactant in order to enhance surface conductivity by the adsorption of moisture from the air. In most of the experiments, temperature and relative humidity were controlled to $20 \pm 2{ }^{\circ} \mathrm{C}$ and $50 \pm 5 \%$, respectively.

In Chapter 4, the macroscopic behaviour for the initiation and halting of flow, and steady-state flow is analysed using the models of the electrical clamping force at a single contact. Chapter 4 starts by reviewing the theory for these models.

In order to analyse experimental data from bulk measurements, it is necessary to translate the single contact force models into a macroscopic representation of the bulk stresses in the material. This transformation is complicated for two reasons. First, the interparticle electrical clamping forces in an assembly of particles will be dependent on direction. This is because contacts between surfaces on equipotential lines do not generate electrical clamping forces. The electric potential at a particular contact will depend on the current paths through the assembly, which will in turn depend on the packing arrangement of the particles with respect to the positions of the electrodes. Therefore, the exact 
distribution of the electrical clamping forces can only be calculated from a distinct element analysis of the current flow through the bed. This analysis is straight forward for the contacts between the layer of particles next to a wall where the randomness of current paths is avoided. For internal contacts in a randomly packed bed of particles, the calculation of the exact values of the electrical clamping force is complicated. However, by making simplifying assumptions about the net distribution of current in the bed, an expression is developed for the average electrical clamping stress acting on an internal plane.

The second complication in transforming the single contact force models into a bulk stress model is the question of how to describe the physical manifestation of the electrical clamping forces. They may act as an additional externally applied stress on the material which does not change the frictional properties of the material. Alternatively, the electrical clamping forces may produce a cohesion in the material by changing the adhesive nature of the contacts without changing the net stress state of the material. The two approaches require different methods of analysis. The former approach is by far the simpler of the two, and in previous investigations on electrostatic precipitation and electrofluidised beds, this interpretation has been taken $[15,19,20]$. However, the choice of approach is more than purely a matter of mathematical representation. It has significant implications on the physical explanation of the behaviour of granular materials in electric fields. In the EVS, the formation of stable arches upon halting of the flow cannot be explained by modelling the effect of the electrical clamping force as an applied external stress. The formation of a stable stress-free surface can only be explained by the creation of cohesion in the material. Both the external stress and cohesion interpretations are used in Chapter 4 to analyse the behaviour in the shear cell and arching experiments.

An analysis of steady-state flow in the EVS is also presented in Chapter 4. However, because of the complex nature of the electric fields in the electrode configurations of the EVS, the simplifying assumptions about the distribution of the electrical clamping stress between the electrodes are not valid for the EVS. Therefore, in the first instance, a less rigorous analysis of the steady-state flowrate was undertaken than the analyses of yield stresses and arching. The effect of the electric fields on flow was modelled in an analogy with the retarding effect of interstitial gas 
pressure gradients on discharge of fine granular materials from hoppers and bins [33]. A semi-empirical model was developed to describe the effect of electric field strength on flowrate based on the general form of the electrical clamping force models.

The analyses of the bulk behaviour in Chapter 4 show that there is a large discrepancy between the experimental measurements and the theoretical predictions based on the single contact electrical clamping force models. As the single contact electrical clamping force models have never been verified by direct measurement, it is difficult to conclude whether the modelling error lies in the macroscopic transformation of these single contact models, or in the single contact models themselves. However, a fresh examination of the single contact models raises doubts concerning the assumption about contact deformation in the models. Because the electrical clamping force is very sensitive to the contact area, other patterns of deformation could lead to substantially different magnitudes of force. The effect of the electrical clamping force on single contact friction is also not clear from the measurement of macroscopic behaviour and significantly affects the mathematical interpretation of single contact forces and bulk stresses. Based on these views of the electrical clamping force models, it was decided that direct measurement of the electrical clamping force at a single contact was necessary to verify the models, and if necessary to develop new models. Therefore in the final stages of this research, the approach changed from a macroscopic level to a microscopic evaluation of the electrical clamping force. This work is presented in Chapter 5.

Compared to the relatively simple experimental plan for the original bulk scale investigation, the measurement of single contact forces was a significant challenge. This was accomplished by modifying a Nanotest device which was designed for measuring surface hardness at nanometre penetrations under very small compressive loads [34]. Chapter 5 describes the measurement of electrical clamping forces using this device. These forces were measured for the detachment of glass spheres $(1.9$ and $3.7 \mathrm{~mm}$ diameter) from contact with a polished stainless steel surface for a range of applied electric field strengths. The experimental measurements of detachment force are compared with the predictions of the electrical clamping force models. The results confirm the trends shown by the analysis of macroscopic behaviour in Chapter 4. Chapter 5 also describes 
attempts to model contact deformation by the electrical clamping force using values of contact size inferred from measurements of contact resistance. This work was inconclusive because there was insufficient data on the surface properties of the particles to calculate contact areas with sufficient accuracy.

In Chapter 6, the main results reported in the previous chapters are summarised and used to compile the conclusions of this research. Based on these conclusions, recommendations are made in Chapter 7 for the direction of future work on modelling the effect of electrical clamping forces on the mechanics of granular material. 


\section{REVIEW OF PREVIOUS INVESTIGATIONS OF THE ELECTRICAL CLAMPING FORCE}

\subsection{INTRODUCTION}

Electric current is constricted at the contact between two non-conforming resistive bodies (or between a resistive body and a conductor), which results in increased electrical resistance at the contact point [35-38]. It is recognized that this high electrical resistance produces greatly enhanced electric fields across the surfaces in the gap ouside the contact region. In consequence, a force is generated between the two bodies. Several experimental and theoretical investigations have been carried out to evaluate this force, termed the electrical clamping force. These are reviewed below.

All of the previous experiments involved measurements of bulk behaviour in a densely packed assembly of particles, e.g. shear stress, pressure drop, from which values of the electrical clamping force at a single contact were inferred. In general, the investigations were carried out to provide further understanding of particulate behaviour specifically in electrostatic precipitators and electrofluidised beds. In some of the previous studies, theoretical models of the electrical clamping force have been developed. These are mentioned briefly in this Chapter. A detailed review of the models is given in Chapter 4 where they are used to analyse experimental data for the macroscopic behaviour of beds of particulate solids in electric fields. Previously, there have not been any direct experimental measurements of the electrical clamping force for a single contact. This was addressed in the later stages of this work as given in Chapter 5 .

\subsection{REVIEW OF PREVIOUS WORK}

The work of McLean [15]

McLean [15] investigated the adhesion of the dust cake collected on the passive electrode in electrostatic precipitators. In his experiments, McLean measured the force required to separate a metal plate from a packed 
bed of fly ash under application of an electric field. The electric field was applied across the bed parallel to the direction of the detachment force. The experiments were carried out at room temperature and atmospheric pressure.

The detachment stresses were measured for two samples of the same material. There was a large scatter in the detachment force measured at each value of electric field. Detachment stresses of approximately 10 to $180 \mathrm{~N} \mathrm{~m}^{2}$ were measured for applied electric field strengths of approximately $0.2 \times 10^{6}$ to $1.3 \times 10^{6} \mathrm{~V} \mathrm{~m}^{-1}$, respectively. These measurements are based on zero tensile stress when no electric field is present. McLean concluded that the detachment force was approximately linearly dependent on the applied electric field strength.

McLean developed theoretical models for the electrical clamping force which are discussed in Chapter 4. However, he did not compare the theoretical predictions from these models with the experimental data.

\section{The work of Robinson and Jones [32]}

Robinson and Jones [32] measured the yield shear stress between the surface of a packed bed of particles and a moveable brass plate under application of electric fields perpendicular to the plate. Glass beads and sand of particle diameter 600 to $710 \mu \mathrm{m}$ were used, with bed heights of $12.7,25.4$, and $50.8 \mathrm{~mm}$. The mechanical normal load applied to the bed was also varied by loading the brass plate with weights.

For a constant value of electric field, the yield shear stress was found to vary linearly with the applied mechanical normal stress. The bed height had no effect on the yield loci so it could be concluded that the measured shear stresses characterized the forces at the interface between the bed surface and the brass plate, and were independent of forces in the bulk of the bed.

On application of electric fields of 390 and $790 \mathrm{kV} \mathrm{m}^{-1}$, the yield loci were shifted parallel to the zero field locus, with increasing shear stresses required for increasing field strengths. This behaviour was observed for both the sand and the glass ballotini. However, the magnitude of the shifts in the yield loci with applied field were much less for the 
sand than for the glass ballotini.

Since the angle of wall friction for each material appeared not to be affected by the electric field, Robinson and Jones assumed that the electric field was producing an additional applied stress on the bed, in addition to the mechanically applied load. Robinson and Jones calculated values of the electrical stress by fitting the Coulomb yield function to the experimental data. These values of electric stress varied approximately linearly with the applied electric field strength. This quantitative analysis is discussed in more detail in Chapter 3.

Robinson and Jones did not develop theoretical models for the single contact electrical clamping force.

The work of Johnson and Melcher [18]

Johnson and Melcher [18] investigated the behaviour of electrofluidised beds. They measured the pressure drop overshoot at incipient fluidisation and made qualitative observations of the bed appearance. Johnson and Melcher examined beds of sand of $0.5 \mathrm{~mm}$ average diameter fluidised with air in a square column. The relative humidity of the air was controlled at an unspecified room temperature.

Two electrode configurations were used. In one configuration, two plate electrodes were situated on opposite walls of the column producing an average electric field in the direction perpendicular to the gas flow. This arrangement is known as the cross-flow configuration. In the other configuration known as co-flow, mesh screens were placed in the column across the flow path of the gas and a voltage was applied between the screens to produce an average electric field in the direction of the gas flow. The behaviour of the bed was much more uniform in the cross-flow configuration than in the co-flow configuration so the former configuration was investigated more extensively.

The pressure drop was measured for the cross-flow configuration as a function of the applied electric field as the bed was fluidised. The measurements were made at a relative humidity of $99 \%$. The electric field caused a pressure drop overshoot at incipient fluidisation which was larger than the pressure drop overshoot measured without an applied field. 
Johnson and Melcher found that the magnitude of the pressure drop overshoot was approximately linearly dependent on the applied electric field strength. From a force balance on the bed, the pressure drop at incipient fluidisation was used to calculate the shear stresses at the electrodes. In a very approximate comparison, they found that the magnitudes of the shear stresses were 30 times the value which would be predicted by the electrostatic attractive force in a homogeneous dielectric. The magnitude of the electrostatic attractive force is considered as part of this work in Chapter 4.

At the onset of bubbling when the particles lost intimate contact, the pressure drop across the bed was no longer affected by the electric field. However, the appearance of the bed was significantly altered by the electric field depending on the relative humidity. For the cross-flow configuration at relative humidities greater than about $50 \%$, the application of an electric field caused the particles to form strings which tended to follow the field lines and suppress the bubbles. At a relative humidity of $8 \%$, the behaviour of the bed was very different from the higher relative humidities and was also highly irreproducible. Because the surface conductivity of the particles was reduced at the low relative humidity, the charge relaxation time of the particles became much longer and tribocharging of the particles caused electrical effects to modify the bed behaviour even without application of an electric field. Without an applied electric field, the bed channelled and a thin coating of particles formed on the walls. With an applied field, a thick layer of particles formed on each electrode.

\section{The work of Dietz [19], and Dietz and Melcher [20,39]}

Dietz [19], and Dietz and Melcher [20,39] carried on the work of Johnson and Melcher [18] on electrofluidised beds. They used three types of experiments to make measurements of the bulk behaviour of electrofluidised beds: Pressure Drop Overshoot, Raining Bed, and Viscometry. They then developed a method of analysis from which the electrical clamping force at a single contact could be inferred, and compared it to a theoreticai model which they developed based on the current constriction phenomenon.

The experiments were done with sand, glass beads and styrofoam beads. The sand and glass beads had particle sizes ranging from $0.1 \mathrm{~mm}$ to $0.6 \mathrm{~mm}$, with 
each test batch having a relatively narrow size distribution. The styrofoam beads had a particle diameter range of 1.5 to $2.5 \mathrm{~mm}$. In order to eliminate tribocharging of the particles, the surface conductivities of the materials were increased by using air for fluidisation with relative humidity greater than $80 \%$ and by coating the styrofoam beads with an anti-static agent. By maintaining the value of the surface conductivity sufficiently high to eliminate tribocharging, it was found that the experimental results were not affected by the actual value of the surface conductivity.

\section{Pressure Drop Overshoot}

These experiments were carried out with a cross-flow electrode configuration and similar results to Johnson and Melcher [18] were obtained.

\section{Raining Bed}

The pressure drop apparatus was modified slightly to measure the electric field strengths required to suspend the bed against an upper screen as a function of air flowrate. This technique has been used in other studies of fluidisation to evaluate the role of interparticle forces $[40,41]$. In Dietz and Melcher's experiments, a screen was placed above the bed and the air flowrate was increased until the bed was held against the screen. A voltage was then applied between the two electrodes in cross-flow configuration and the flowrate reduced until particles began to fall away from the bed. At low field strengths, the particles 'rained' off of the lower surface of the suspended bed individually or in small clumps. At high field strengths, large sections of the bed fell away.

Values of the electrical clamping forces could be interpreted from these experiments in two ways. When the bed was completely supported by the field with no air flow, the electrical shear stress at the electrodes must be supporting the weight of the bed. Also with no air flow, when the field was reduced to a value such that particles just started to rain from the surface, the electrical clamping force on each particle must equal the gravitational force. Estimation of the electrical clamping stresses from these force balances was not straight forward because not all of the materials could be supported by the electric field at zero air flowrate 
below the sparkover limit of the bed. For these materials, the values were obtained by extrapolating the data to zero air flowrate.

\section{Viscometry}

In their third type of experiment, Dietz and Melcher used a Couette viscometer to measure the shear stress at the wall of an electrofluidised bed at the point of incipient fluidisation. Again, the cross-flow electrode configuration was used. The curves of shear stress as a function of applied voltage from these experiments could be characterized by two regimes. For low electric fields, the shear stress was relatively independent of the applied voltage. The shear stress fluctuated widely with time and increased with increased shear rate. For high electric fields, the bed became relatively rigid in structure so that the shear stress was independent of shear rate and increased with increasing electric field.

Dietz [19], and Dietz and Melcher [20,39] compared their experimental data with theoretical predictions based on models of the single contact electrical clamping force developed by Dietz [19]. They found good agreement with the bulk experimental data. However, their formulation of the bulk electrical stress is incorrect. The correct formulation is discussed in Chapter 4.

The work of Moslehi and Self [16]

Moslehi and Self [16] developed a rigorous mathematical formulation of the single contact electrical clamping force. The basic theory and assumptions behind their model are similar to those of McLean [15] and Dietz [19].

Moslehi and Self did not compare their force model to experimental data. Their model will be explained in greater detail in Chapter 4, where it is used to analyse bulk measurements, and in Chapter 5, where it is assessed against direct measurements of the single contact force.

The work of Kendall [31]

Recently, Kendall [31] has developed a model for the electrical clamping force using a different approach to those discussed above. Kendall uses an 
energy balance approach similar to that used in linear elastic fracture mechanics for the calculation of the electrical clamping force for an annular crack in a cylinder across which an electric field is applied. This approach is thermodynamically consistent as it considers the balance of strain energy and the work of the electric field. However, the geometry used by Kendall is different from the geometry used in the experiments carried out in this work, i.e. two spheres in contact, and a sphere in contact with a plane. The prevailing electric field pattern in the cylindrical geometry is very different from that in the spherical geometry. For this reason, Kendall's model is not considered in the analyses of experimental results in this work. However, Kendall's approach is highly interesting and merits a detailed discussion which is presented in Chapter 5.

\subsection{CONCLUSIONS}

The previous experimental work on the electrical clamping forces showed that these forces significantly alter the behaviour of packed beds of particulate solids. The generation of large stresses was shown by direct measurement and by inference from pressure drop measurements on bulk materials. Of significant interest is the sensitivity which highly resistive particles like sand and glass showed to relative humidity in the work of Johnson and Melcher [18]. For relative humidities above 50\%, tribocharging of the particles was eliminated and the bed behaviour was relatively insensitive to the relative humidity. This behaviour indicates that conduction of electric current through the adsorbed moisture layer on the particle surfaces dominated over volume conduction.

The work of Robinson and Jones [32] shows another interesting phenomenon. The same applied field strength increased the wall yield stress of glass beads more than sand of the same particle size range. This difference may be due to a number of factors such as different particle packing density, different interparticle contact size and shape, different surface roughness, or different conductivity. It is important to understand the influence of each of these factors on the electrical clamping forces.

The experimental work reviewed in this chapter all has two common factors in the experimental design which limit the information which can be gained 
from the data. The bulk measurements of stresses were all related to the stresses acting between the electrode walls and the bed. No measurements were made of internal stresses in the bed. The second limitation of the experiments was the use of macroscopically uniform electric fields in one direction only. Johnson and Melcher made qualitative observations with co-flow electric field configurations but no quantitative measurements. Also, the co-flow electrode designs were significantly different from the cross-flow designs so the behaviour in the two configurations is not directly comparable. The effects of electric field direction and gradient were not examined in previous work on the electrical clamping forces.

Some researchers have developed quantitative models for the electrical clamping force. None of the models have been verified by direct measurement as this requires single contact experiments which are difficult to conduct due to the sophistication and sensitivity of the equipment required [42]. Dietz [19], and Dietz and Melcher [20,39] used measurements of bulk behaviour to indirectly verify their models, but their methods of analysis are flawed. In order to model the flow behaviour of granular materials in electric fields, a formulation is required for transforming the single contact models into a description of electrical stress in the bulk material. The electrical stresses can then be superimposed on the mechanical stresses in the material. The next two Chapters deal with the interpretation of bulk behaviour based on the single contact models of the electrical clamping force. In Chapter 3, experimental measurements of the bulk behaviour of granular materials in electric fields are presented. The results of these experiments are used to evaluate the quantitative analysis in Chapter 4. 


\section{CHAPTER 3}

OBSERVATIONS OF MACROSCOPIC BULK BEHAVIOUR OF PARTICULATE SOLIDS WITH ELECTRIC FIELDS

\subsection{INTRODUCTION}

The objective of this research was to model the effect of electric fields on the flow of densely packed particulate solids, in particular, flow of solids in the EVS. As outlined in Chapter 1 , the first step in the research was to carry out a programme of experiments in order to make observations of the behaviour of granular material in electric fields. The results of these experiments acted as a guide for modelling, and provided data for verification of the models at a later stage.

The intriguing behaviour of solids in the EVS highlighted three aspects of the flow which required further research. These are listed below:

i) Initiation and halting of the flow

ii) Steady-state flow

iii) Transient flow

The first two aspects of the flow modelling were addressed in detail in this work. The third aspect attracted some attention on the characterization of the time constants, but no detailed analysis of the dynamic response was carried out. Details of the work are described below.

Observations of particle movement in the region of the electrodes of the EVS show that when the flow is halted, the material forms stable arches over the individual mesh openings of the downstream electrodes. As these mesh openings are made larger, higher electric fields are required to stop the flow. In order to predict the conditions at which flow of the material will just occur, two pieces of information are required: the stress state in the material and the yield function. Therefore, in order to model the conditions for initiation or halting of flow in the EVS, models for the stresses in an arch and the yield function of the material as a function of electric field are required. For this purpose, two experimental apparatus were used: a shear cell, and a wedge-shaped hopper for arching.

The yield function of a material describes the limiting conditions for the 
normal and shear stresses which will cause the material to flow. There are several correlations available to describe the yield behaviour of granular materials [43,44]. The correlation which best describes the yield function of a particular material is found by measuring the yield stresses in the material using a shear cell [45]. As noted in Chapter 2, Robinson and Jones [32] had already measured the wall yield loci for sand and glass spheres with electric fields and found the Coulomb yield function to be suitable. These results are discussed in more detail in this chapter. As part of this work, the effect of electric fields on the internal yield of a granular material was measured in a shear cell for a packed bed of glass spheres.

The yield function provides information about the limits of stresses for failure of a material. To predict whether these limits are being exceeded at any point so that bulk failure occurs, the stress distribution in the particular geometry of interest must be known. This is difficult to measure, except at a wall where load cells can be installed without disturbing the material. Consequently, several theories have been developed to predict the mechanical stress distributions in various geometries $[46,47]$. In particular, the stresses in a wedge-shaped or conical hopper have been studied to predict whether arching will occur at the orifice of the hopper [48-50]. Observations of flow in the EVS show that application of an electric field can produce arching and halt flow, where mechanical stresses alone are insufficient. In this work, a wedge-shaped hopper with electrodes along the side walls was used to study arching under the application of an electric field. This simple electrode system was used to measure the voltages required to halt the flow of glass spheres by the formation of an arch at the orifice of the hopper.

The methods and results of the shear cell and arching experiments are presented in detail below. In order to model the halting and initiation of flow, the effect of the electric field on the yield function and on the stresses must be considered. On both these points, the interpretation of the experimental results is discussed. The conclusions are used in Chapter 4 to develop quantitative models for the halting and initiation of flow by the formation of arching.

The second aspect of flow of particulate solids in electric fields which was considered as part of this research is the steady-state relationship 
between electric field strength and flowrate. Measurements of the flowrate as a function of the electric field were carried out in the EVS itself with the electrode configurations shown in Fig. 1.1. The first objective of these experiments was to assess the relative sensitivity of the EVS performance to the design variables, e.g. column dimensions, electrode design, distance between the electrodes, in order to guide the future work. The results of these experiments are presented and discussed below. The experimental data is considered further in Chapter 4 where a quantitative model for the steady-state flowrate is developed.

Finally, transient flow behaviour of granular materials under changing electric fields is considered. Measurements of time constants and flowrates in the EVS are presented and discussed below.

\subsection{INTERNAL AND WALL SHEAR STRESSES AT FAILURE}

The measurement of the shear stresses at failure which are generated internally and at a wall for a packed bed in an electric field was undertaken to characterize the effect of the electrical clamping forces on the stresses at failure and on the flow of granular materials. The electrode geometries of the EVS, as shown in Fig. 1.1, are complicated and produce complex and nonuniform electric fields. In order to test some basic hypotheses about the interactions of the electrical and mechanical forces, it was desirable to use a geometrically more simple and well-defined electrode system of a packed bed between two parallel plate electrodes. The measurement of internal shear stresses was undertaken as part of this work in a collaborative effort by Martin et al. [51] using a box type shear cell whose upper and lower surfaces consisted of the plate electrodes. These experiments are discussed in detail below. Wall shear stresses for a similar system had previously been measured by Robinson and Jones [32] and their results are presented here for comparison.

The measurement of internal shear stresses was carried out with a modified box-type shear apparatus. The shear box itself is made of electrically insulating epoxy resin to enable the application of electric fields, as shown in Fig. 3.1. The two halves of the box are constructed so that the surface area in contact between them is very small and remains constant during shear. The shear force between these surfaces is measured with the 
cell empty and subtracted from the shear measurements with material in the cell in order to obtain a more accurate measurement of the material shear stress. An earthed brass electrode is fixed into the bottom of the lower half of the box, and a high voltage brass electrode is attached to the bottom of an insulating lid which rests on the material during a shear test. The shear box has an inside side length of $100 \mathrm{~mm}$ and each half of the box is $12.5 \mathrm{~mm}$ high.

To perform a test, the shear box is initially over-filled with material so that a level suface can be created by scraping away excess material with a rigid blade. The lid is situated on top of the material in the shear box and weights are placed on the lid for the application of varying normal mechanical loads. During shear, the upper half of the shear box remains fixed, while the lower half of the box is pulled at a constant strain rate of $1.2 \mathrm{~mm} \mathrm{~min}^{-1}$. The upper half of the shear box is attached to a strain gauge load cell for measurement of the peak tangential shear force. Electric fields are applied to the material during shear by setting a voltage potential across the electrodes using a DC high voltage supply. The room conditions were controlled to $20 \pm 2^{\circ} \mathrm{C}$ and $50 \pm 5 \%$ relative humidity.

The test material used in this work was prepared by sieving out soda glass ballotini in the size range 600 to $710 \mu \mathrm{m}$ diameter from the bulk material. The selected material was lightly coated with surfactant by mixing about 1 $\mathrm{kg}$ of glass ballotini at a time into $5 \mathrm{~L}$ of $1 \%$ aqueous solution of anionic surfactant, and then pouring the mixture through a sieve and allowing the glass ballotini to dry on the sieve at room temperatures of $20-25^{\circ} \mathrm{C}$. The glass ballotini was coated with surfactant in order to increase the surface conductivity, and thus ensure fast charging times with surface conductivity dominating over volume conductivity.

The effect on resistivity of coating the glass ballotini with surfactant was measured using a resistivity cell consisting of copper plates, $50 \mathrm{~mm} \mathbf{x}$ $50 \mathrm{~mm}$, spaced $10 \mathrm{~mm}$ apart, embedded in blocks of Perspex. The cell was loosely packed with glass ballotini between the copper plates, and the resistance across the plates was measured with a Wayne Kerr electrical bridge. These measurements were done before the temperature and humidity control equipment was installed, so different conditions prevailed from the shear cell experiments. The temperature and relative humidity for the resistivity measurements were $23^{\circ} \mathrm{C}$ and $65 \%$ respectively. The bulk 
resistivity of the glass ballotini, $\rho_{b}$, was calculated from the measured resistance, $R_{b}$, using the following relationship:

$$
\rho_{b}=\frac{A}{L} R_{b}
$$

where $A$ is the area of one electrode and $L$ is the distance between the electrodes.

The resistivities of uncoated and coated glass ballotini were 3.9 and 0.13 G $\Omega$ m respectively, which shows that the surface conductivity of the glass ballotini was significantly increased by the surfactant coating.

In the wall shear experiments of Robinson and Jones [32], a brass sled was pulled over the surface of a bed of material and the traction was measured as a function of the normal load with and without the application of an electric field. In their experiments, the brass sled was earthed and a plate at the bottom of the bed formed the high tension electrode so that the macroscopic field was perpendicular to the direction of shear force. The normal load was generated by putting weights on the sled and the static yield shear was determined by loading a pulley attached to the sled with weights until the sled yielded. Robinson and Jones performed these experiments for glass ballotini with a particle size range of 600 to $710 \mu \mathrm{m}$ for bed heights of 12.7 to $50.8 \mathrm{~mm}$ at ambient temperature and humidity conditions. It is interesting to note that Robinson and Jones did not control the relative humidity in their experiments. When monitoring the current flowing through the bed between the electrodes they found that it varied considerably from day to day. However, the shear stresses measured under the same conditions showed almost no variations.

The results of the shear stress experiments are shown in Fig. 3.2 for internal shear and in Fig. 3.3 for wall shear. The experimental data are plotted in the form of traditional yield loci with shear stress as a function of normal mechanical load. Three similar field strengths were used for the internal shear and the wall shear measurements. Fig. 3.2 shows the yield loci for internal shear for zero field, $470 \mathrm{kV} \mathrm{m} \mathrm{m}^{-1}$ and $800 \mathrm{kV} \mathrm{m}^{-1}$. Fig. 3.3 shows the yield loci for wall shear for zero field, $390 \mathrm{kV} \mathrm{m}^{-1}$ and $790 \mathrm{kV} \mathrm{m}^{-1}$.

In both internal and wall yield, the electric field causes a shift in the 
yield loci approximately parallel to the zero field locus, which suggests that the angles of wall and internal friction remain unchanged with application of an electric field. On this basis, Robinson and Jones [32] suggested that the electrical stress acts as an external load in addition to the normal mechanical load so that the yield shear stress is described by the Coulomb yield function [44] modified such that

$$
\tau=\mu\left(\sigma_{\mathrm{m}}+\sigma_{\mathrm{e}}\right)+\mathrm{c}
$$

where $\tau$ is the yield shear stress, $\sigma_{\mathrm{m}}$ is the normal mechanical stress due to the weight of the sled, $\sigma_{e}$ is the externally applied electrical stress, $\mu$ is the friction coefficient, and $c$ is the natural cohesion/adhesion of the material in the absence of an electric field. In this interpretation, the effect of the electric field is to shift the zero field yield locus horizontally by an amount $-\sigma_{e}$.

Alternatively, the effect of the electric field on the yield stresses may be described as producing increased cohesion between the particles without increasing the normal load. In this case, the bulk yield criterion for a naturally cohesionless material would take the form

$$
\tau=\mu\left(\sigma_{m}\right)+c_{e}
$$

where the electric field causes the zero field locus to shift vertically by an amount $c_{e}$.

Equations (3.2) and (3.3) were fittedto the experimental data shown in Fig. 3.2 and 3.3 and the resulting values of $\sigma_{e}$ and $c_{e}$ are shown in Table 3.1. From the experimental data in Fig. 3.2 and 3.3, it is impossible to prove which yield function appropriately describes the effect of the electric field. Both equations (3.2) and (3.3) describe the effect of the electric field on the yield shear stress equally well. All the experimental and theoretical work in the literature interpret the bulk manifestation of the electrical clamping force as an externally applied normal load $[15,16,19,20,31,32,39]$. However, none of the experimental data show this unequivocally. If the electrical clamping forces manifest themselves as an applied normal stress, the bed should consolidate as with an applied mechanical normal stress. However, for high elastic modulus materials like glass and sand which have generally been used in the bulk experiments, the 
bulk consolidation of the sample would be small and not evident to the eye, except for very large applied stresses.

The implications of choosing the correct description for the effect of the electrical clamping forces on yield will be discussed in the next section on arching, where the physical significance of equations (3.2) and (3.3) will become apparent. Both alternatives will be used to analyse the experimental results using the single contact models of the electrical clamping force in Chapter 4.

\subsection{ARCHING IN HOPPERS}

An analysis of arching was undertaken because observations of particle movement in the region of the electrodes in the EVS show that halting of the flow occurs by the formation of stable arches over the mesh openings of the downstream electrode. As this is observed for materials such as glass ballotini which are cohesionless, the arches must be supported by the electric field. In order to understand the arching process better and provide a well-defined system for modelling, a wedge-shaped electrode-hopper system was used for the arching experiments. The hopper was used to measure the voltages required to prevent material from flowing by the formation of a stable free surface at the orifice. The results of these experiments are also presented in Martin et al. [52].

A schematic diagram of the hopper is shown in Fig. 3.4. The hopper was constructed with adjustable orifice width and hopper half angle in order to measure the voltage required for arch formation at the orifice as a function of orifice width and hopper half angle. The front and back walls were made of Perspex so the behaviour of the material, especially at the orifice where arching occurred, could be directly observed. A depth of 12 $\mathrm{mm}$ was chosen for the hopper in order to give near two-dimensional flow and stress distributions. The side walls consisted of metal strips of $12 \mathrm{~mm}$ width, forming the electrodes. Two types of metallic surfaces were used for the electrodes in order to provide partially smooth or partially rough conditions: a polished stainless steel sheet, and a brass sheet roughened by milling $0.5 \mathrm{~mm}$ deep triangular grooves into the surface, respectively. The degree of roughness of the surfaces was quantified by the angles of wall friction as described below. Soda glass ballotini of particle 
diameter 600 to $710 \mu \mathrm{m}$ was used as the test material. The glass ballotini was coated with surfactant in the same way as described for the shear cell experiments in order to increase the surface conductivity. The experiments were conducted in a humidity and temperature controlled environment at $50 \pm 5 \%$ relative humidity and $20 \pm 2{ }^{\circ} \mathrm{C}$. The distance between the vertex and the top surface of the material in the wedge was kept constant at $0.545 \mathrm{~m}$ in all the experiments.

The angle of internal friction, $\delta$, for the glass ballotini was obtained from the yield loci measured in the internal shear cell experiments in Section 3.2. By fitting the experimental data from the yield loci in Fig. 3.2 to equation (3.1), where in this case the coefficient of friction $\mu$ equals $\tan \delta$, the angle of internal friction is $20^{\circ}$ with or without an electric field. The angles of wall friction for the two different electrode surfaces were determined by the following method. A single layer of glass ballotini was glued to a Perspex block. The layer of particles was then placed on the electrode, which was originally in a horizontal position. The electrode was fixed at one end and the other end of the electrode was then raised to provide an inclined surface. The angle of wall friction $\phi$ was taken to be the angle of inclination from the horizontal of the electrode at the point of initiation of particle sliding. The angles of wall friction were $16^{\circ}$ for the polished electrode and $20^{\circ}$ for the grooved electrode.

To measure the holding voltage, i.e. the minimum voltage required to hold the material which is already in a static state, the hopper was filled with the material and the maximum possible test voltage was applied, i.e. the voltage just below the electrode sparkover voltage. The flap door below the outlet of the hopper was then opened. If the material did not flow, the test was repeated by decreasing the voltage step-wise by $1 \mathrm{kV}$ until flow was obtained. The lowest voltage at which material did not flow was taken as the static holding voltage. Three hopper half angles, $0^{\circ}, 10^{\circ}$ and $20^{\circ}$, and several orifice widths in the range 6 to $16 \mathrm{~mm}$ were used.

The experimental results for the holding voltage as a function of arch span are shown in Fig. 3.5 and 3.6 for the smooth and rough walled hoppers, respectively. The reproducibility of the experimental data was found to be $\pm 0.5 \mathrm{kV}$. The experimental results from Fig. 3.5 and 3.6 show that the holding voltage increases with gap width, which is expected since the 
normal load on the arch increases as the gap width increases and higher electrical clamping forces are needed to balance the increased mechanical forces on the arch. Comparing Fig. 3.5 to Fig. 3.6 shows that the holding voltages for the smooth wall are higher than those for the rough wall. This behaviour is also expected since the mechanical lifting force from the walls decreases as the walls become smoother and must be compensated for by higher electrical forces. The experimental results show little dependency of holding voltage on the hopper half angle. For the smooth walled hopper, the results in Fig. 3.5 show no trend at all for holding voltage as a result of hopper half angle. For the rough walled hopper, the results in Fig. 3.6 show that holding voltage increases for an increase in hopper half angle from $0^{\circ}$ to $10^{\circ}$, but there is no subsequent increase in holding voltage when the hopper half angle is increased from $10^{\circ}$ to $20^{\circ}$. Although it is expected that increasing the hopper half angle should increase the normal load on the arch and therefore require increased holding voltages, the expected degree of sensitivity of this relationship is not observed. These relationships between the holding voltage and the experimental variables are analysed in Chapter 4 according to the theoretically predicted stress distributions, based on the single contact models for the electrical clamping forces.

The holding voltages shown in Fig. 3.5 and 3.6 were measured with the bed in a state of static stresses. By allowing the material to flow with zero voltage and then applying a voltage, the holding voltages for the bed in a state of dynamic or flowing stresses were measured. For the experimental system used here, the dynamic holding voltages were all greater than those measured statically. In fact, only at a very few small orifice widths, all less than $10 \mathrm{~mm}$, could initially flowing material be halted at voltages less than the sparkover voltage. Therefore, no quantitative results are presented for dynamic holding voltages. A flowing material must dilate from the filled state and therefore has a less dense packing than in the filled state. This would produce a reduced density of the electrical clamping forces and may explain the need for higher holding voltages for a flowing material. The sliding contacts in the shear bands at the walls in flowing material may also affect the magnitude of the electrical clamping forces.

The observed shape of the arch free surface and the process of failure of this surface can be described by considering three voltage ranges, in which 
the voltage was initially set equal to, less than, and higher than the static holding voltage for a particular orifice width. Slight differences in behaviour were also observed between the partially smooth and partially rough wall cases.

When the initial voltage was set equal to the holding voltage in the smooth wall case, the material froze as a distinctly convex arch at the orifice. This convex arch is shown schematically in Fig. 3.7. In the rough walls, the surface was generally flat with a tendency to become slightly convex as the hopper half angle was increased. In both cases, if the voltage was then slowly lowered in $1 \mathrm{kV}$ steps, small clumps of particles sometimes fell away from the material at the orifice, but the rest of the bed remained frozen until a sufficiently reduced voltage was attained at which continuous flow suddenly started. These observations indicate that the shape of the free surface at the hopper outlet is determined by the directions of the electric field lines at the tip of the electrodes which necessarily differ from the field lines in the bulk of the hopper.

When the initial voltage was set slightly lower than the holding voltage by about $1 \mathrm{kV}$, failure of the material in the smooth walled hopper occurred as slow, but steady, rupturing of complete particle chains from the outer layer of the convex arch. A chain would first break away from one electrode tip and then fall away from the surface until it broke at the other electrode tip. This indicates that cohesive forces were acting to keep the particles in the chain together. Movement in the bed was observed only in the immediate vicinity of the orifice, where the material moved down slowly to take the place of the lost particles. As the initial test voltage was set increasingly lower than the holding voltage, the rate of failure increased with thicker layers of particles falling away from the arch surface, until at a sufficiently low voltage, continuous flow occurred. This continuous flow was still slower than the flow at zero voltage. This was particularly noticeable by the slow movement of the particle layers along the electrode walls near the orifice. In the rough walls, there was no intermediate voltage range which resulted in slow rupturing of chains from the arch surface. When the voltage was initially set slightly lower than the holding voltage in the rough walled hopper, failure occurred as continuous flow from the orifice, although at a slower rate than without an applied field. 
The effect of setting the initial voltage higher than the holding voltage could not be tested in the smooth walled hopper because the holding voltages were very near the maximum test voltages. The holding voltages in the rough walled hopper were lower than those for the smooth walled hopper as shown by comparing Fig. 3.5 and 3.6. Therefore, it was possible to test intitial voltages at least $2 \mathrm{kV}$ higher than the holding voltages for most of the orifice widths in the rough walled hopper. Whereas mainly flat surfaces formed at the orifice when the voltage was initially set equal to the holding voltage, higher voltages caused convex arches to form. Once the bed was frozen in this state, lowering the voltage resulted in failure occurring at a voltage which was lower than the holding voltage. This is very interesting behaviour and merits further study. It may be that the higher electrical clamping forces cause deformation of the particle surfaces such that the van der Waal's forces, which persist after lowering the electric field, are enhanced or such that the frictional resistance between the surfaces is modified. It therefore appears that higher fields produce structures which are more stable than those produced at lower voltages even after reducing the field to that corresponding to the holding voltage.

The formation of arches with an electric field illustrates the importance of determining whether the bulk electrical clamping stresses act as externally applied stresses on the material or as cohesive stresses. The schematic diagram shown in Fig. 3.8 shows the Coulomb yield function for a cohesionless material and the Mohr circle describing the stress state of the material at the surface of a stable arch. Because an arch has no material below it pushing up on the arch, the stress normal to the arch surface must be zero. This zero normal stress is the minor principal stress shown by the point $(0,0)$ on the Mohr circle in Fig. 3.8. Since the yield function shows the stress limits for failure, the Mohr circle must lie below the yield function line for the formation of a stable arch. However, the yield function for a cohesionless material passes through the point $(0,0)$, and therefore it crosses the Mohr circle. Thus, it is impossible for a non-cohesive material to form a stable arch. If the electrical clamping forces act to produce an externally applied stress on the material and not a cohesive stress, the material should not be able to form a stable arch. Without cohesion, particles forming the last layer on the free surface should rain down individually. Therefore, the fact that the material in the arching experiments does form stable arches should be 
sufficient proof that the electrical clamping forces act as cohesive forces between particles. However, this cannot be stated conclusively based on these experiments because of the complications of the presence of other electrical forces, arising from the geometry of the orifice, which are additional to the electrical clamping forces acting on the material at the orifice. The discontinuity of the electrode surfaces at the edges of the metal plates produces a very high electric field gradient just at this point. The resulting dielectrophoretic forces may ensure that the particles on the free surface are pushed towards the direction of higher field gradients at the tips of the electrodes, thus providing the key to the stability of the particles at the arch surface. This may be sufficient to maintain stability at the arch surface, if the bulk load of the bed is being supported by electrical clamping forces acting as lifting forces at the walls. From the experimental results presented here, the action of the electrical clamping forces as an externally applied stress cannot be discounted in favour of the electrical cohesion theory. Both approaches are used in Chapter 4 to analyse arching.

\subsection{STEADY-STATE FLOW}

The effect of electric fields on the steady-state flow of granular materials was studied in the EVS itself. The results discussed here were accumulated from various experiments where the potential of the EVS as a valve for solids was evaluated. The work consists of collaborative efforts as documented in Ghadiri et al. [25].

The work on steady-state flowrate in the EVS was carried out with electrode configurations similar to those shown in Fig. 1.1 in two cylindrical columns of 64 and $115 \mathrm{~mm}$ I.D. and in a $200 \mathrm{~mm}$ long by $12 \mathrm{~mm}$ deep rectangular duct. The test rig shown schematically in Fig. 3.9 was used for the experiments. A variety of materials were tested for suitability in the EVS, including detergent powder, crushed coal, $\mathrm{NaCl}$ salt, and FCC powder, but for more detailed analysis of the flow behaviour in the EVS, sand and glass ballotini were used as model cohesionless materials.

When filling the column or storage bin before a test, the flap door is closed in order to obtain the material in a densely packed state. If the electric field is then set to a suitably high level before opening the flap 
door, the particles between the electrodes will be 'frozen', thus supporting the material above the electrodes. No particles will flow when the door is opened except those in the space between the lower electrode and the flap door. In the rectangular duct, it was possible to observe the behaviour of the material inside the duct. When the flow is halted by applying a high electric field, the material forms deep concave arches over the gaps between the wires in the downstream electrode. If the voltage applied to the electrodes is then gradually reduced, a point is reached when the material begins to flow. This is referred to as the static holding voltage, $\mathrm{V}_{\mathrm{sh}}$, as was described in the arching experiments.

Typical results for sand are shown in Fig. 3.10 as a function of height above the upstream electrode. The stress in a granular material in a vessel with frictional walls approaches an asymptotic limit at large bed depths [46]. If the stresses introduced by an electric field prevent flow by overcoming the vertical component of stress in the material, the static holding voltage should then also approach an asymptotic value with increasing bed depth. However, the values of $V_{s h}$ in Fig. 3.10 show poor reproducibility between repeated measurements with no dependence on height of material. Similar scatter is observed for all materials and electrode configurations tested so far, and this may be due to variations in the packing structure, which depend on the filling history, and possible switching between active and passive states on initiation of flow.

If the voltage is reduced to a value which allows the material to flow, a steady-state flowrate is attained which is a function of the applied electric field strength. The flowrates are obtained from the gradient of the weight versus time traces recorded by a load cell from which the collection vessel is suspended. Values for mass flux are then calculated by dividing the total flowrate by the column cross-sectional area. The performances of the EVS for the 64 and $115 \mathrm{~mm}$ diameter columns are shown in Fig. 3.11 for $600-1200 \mu \mathrm{m}$ sand. The electrode configuration shown in Fig. 1.1 was used here, with $10 \mathrm{~mm}$ square openings in the downstream electrode and an inter-electrode gap (IEG) of $36 \mathrm{~mm}$.

The performance of the EVS for the rectangular duct is shown in Fig. 3.12 for sand and glass ballotini both having a particle size range of 600-850 $\mu \mathrm{m}$. In this case, the downstream electrode consisted of parallel wires extending across the duct as shown in Fig. 1.1, with a spacing of $5 \mathrm{~mm}$ 
between the wires, and the upstream electrode consisted of pins extending 1 to $2 \mathrm{~mm}$ into the bed at intervals of $10 \mathrm{~mm}$ along the front and back faces of the duct. The electrodes used in the rectangular duct and the cylindrical columns were made of $0.7 \mathrm{~mm}$ diameter tin coated copper wire.

Fig. 3.11 and 3.12 show that the dependence of mass flux on applied electric field strength is qualitatively similar in the cylindrical and rectangular columns. The dependence of mass flux on applied field is weak at low field strengths, but it becomes increasingly significant at higher applied fields. As the mass flux decreases to below about $50 \%$ of the full flow, it drops rapidly to zero.

The electric current passing through sand $(600-1200 \mu \mathrm{m})$ between the electrodes in the EVS was measured in the $115 \mathrm{~mm}$ diameter column for the electrode configuration shown in Fig. 1.1 with $10 \mathrm{~mm}$ square openings in the downstream electrode and and inter-electrode gap of $36 \mathrm{~mm}$. Figure 3.13 shows that the current is proportional to the applied voltage and thus follows an Ohmic relationship.

The sensitivity of the EVS response to different electrode configurations was examined qualitatively in a series of tests, where the specific effects of the following features were investigated:

\section{(a) geometry of the upstream electrode; \\ (b) separation between the electrodes.}

To check (a), a cross wire (set at $90^{\circ}$ angle) and a single wire were used as the upstream electrode in the cylindrical columns. For (b), the separation between the electrodes, or inter-electrode gap (IEG), was scaled with column diameter. The results of these tests are given in Figs. 3.14 and 3.15 for sand in the size range 600-1200 $\mu \mathrm{m}$ in the 115 and $64 \mathrm{~mm}$ columns, using a $10 \mathrm{~mm}$ grid of $0.7 \mathrm{~mm}$ diameter wires as the downstream electrode.

The effect of the upstream electrode geometry is shown in Fig. 3.14 for the $115 \mathrm{~mm}$ column. The mass flux in the absence of an electric field is nearly the same for each electrode configuration, and does not appear to be affected by the upstream electrode geometry. With an electric field present, however, the mass flux depends on the geometry of the upstream 
electrode. The separation between the electrodes was kept constant at 36 $\mathrm{mm}$ in the results shown in Fig. 3.14, and hence the differences in the results between the two upstream electrode geometries arise from variations in the electric field distribution.

Figure 3.15 shows the results of tests in which the electrode separation in the $115 \mathrm{~mm}$ diameter column has been scaled from $36 \mathrm{~mm}$ to $64 \mathrm{~mm}$ relative to the $64 \mathrm{~mm}$ diameter column. The data are expressed in terms of mass flux so that the results for the two column sizes can be directly compared. As in Fig. 3.14, the mass flux in the absence of an electric field is nearly the same for the two column sizes indicating that here too, with the field applied, the differences in the results are due to variations in the electric field distribution. With an electric field present, Fig. 3.15 shows that the mass flux response for a constant electrode separation of 36 $\mathrm{mm}$ depends on column diameter. On the other hand, changing the electrode separation from 36 to $64 \mathrm{~mm}$ with a constant column diameter of $115 \mathrm{~mm}$ also changes the mass flux response. It is obvious that the configuration of the electrodes used here is such that the electric field distribution in the space between the electrodes is changed by changing the column diameter or electrode separation, thus producing a different flow response; a more uniform field, as in the case of the $64 \mathrm{~mm}$ IEG, gives rise to a sharper fall in the flowrate as the voltage is increased.

It is worth noting that the steady-state flowrate data presented in the figures in this work were obtained by applying a voltage to the material initially in a flowing state at zего voltage. This is termed the dynamic testing method. Alternatively, a static testing method was used. In this case, the voltage was applied while the flap door under the electrodes was still closed so that the material was initially in a static state; flow was initiated by opening the flap door and the steady-state flowrate was then measured. For a given electric field, no difference in flow could be detected between static and dynamic conditions, except for voltages very near to the holding value, where the dynamic holding voltage, $V_{d h}$, was larger than the static holding voltage, $V_{s h}$. The response of the EVS in the region bound by $V_{s h}$ and $V_{d h}$ is found to be unstable, i.e. the flow can stop completely or trickle through irreproducibly. To operate in this region, it is therefore necessary to follow a path as shown in Fig. 3.16.

The effect of the electric field on flowrate requires an appropriate range 
of resistivities to be effective. For highly resistive materials such as PVC and pure quartz, it is difficult to get sufficient current through the particles at voltages below the electrode sparkover voltage. Also, highly resistive materials can behave more erratically due to the effects of static charge on the particles from frictional contact with other surfaces. Therefore, reliable flow control of highly resistive materials cannot be achieved even with modifications to the electrode design such as reducing the mesh opening of the downstream electrode. In these circumstances, the humidity and the presence of surface impurities such as surface active agents can have a significant effect. For example, for the 1.2-2.4 mm silica sand used in these experiments, the bulk volume resistivity is greater than $10^{\circ} \Omega \mathrm{m}$ for relative humidities lower than $30 \%$. The operation of the EVS is then erratic, in that it is difficult to halt the material at voltages below the sparkover voltage. Therefore, the results of the experiments presented here were obtained for relative humidities of $40-60 \%$ at ambient temperatures. When the resistivity of the sand is reduced to approximately $10^{5} \Omega \mathrm{m}$ by increasing the relative humidity to $80 \%$, the EVS operates more consistently, and requires voltages much lower than the sparkover voltage. In the other extreme, too low a resistivity, such as that of a metal powder, generates too much current for the operation of the EVS to be viable.

The above results showed that the flowrate through the EVS is highly sensitive to the electric field distribution between the electrodes which makes it complicated to develop a rigorous quantitative model for flowrate in the EVS. Therefore, in the first instance, a semi-empirical approach to the modelling has been taken. This analysis is given in Chapter 4 .

\subsection{DYNAMIC RESPONSE TO CHANGING FIELDS}

The transient flow characteristics of granular materials in changing electric fields have been studied experimentally in order to assess the full operational range of the EVS. In the first instance, flow with pulsating fields was examined. Secondly, an attempt was made to measure the time constant of the EVS for a simple step change in applied field. Finally, the response of the EVS to Proportional-Integral-Differential (PID) control was investigated. The results of these experiments are given below. This work is taken from various collaborative experimental studies 
which have been documented in Ghadiri et al. [25], Martin et al. [26] and Lo [53].

\section{Flow with Pulsating DC Fields}

In this mode of operation of the EVS, the pulse amplitude, frequency and width can all be varied in order to obtain a desired mass flux. The effects of varying the width and frequency of the puise are shown in Fig. 3.17 and 3.18, respectively, for different materials. In these tests, the pulse amplitude was set at a sufficiently high value to achieve fast and complete cessation of flow on application of a pulse. The data shown in Fig. 3.17 were obtained using the $64 \mathrm{~mm}$ diameter column with electrode configuration shown in Fig. 1.1 with $10 \mathrm{~mm}$ mesh openings. The frequency response shown in Fig. 3.18 was obtained using the narrow rectangular duct shown schematically in Fig. 1.1, with the upstream electrode consisting of pins, and the downstream electrode consisting of parallel wires forming slots of $5 \mathrm{~mm}$ width.

The results in Fig. 3.17 and 3.18 show a trend opposite to that of steady-state flow in a continuous field as shown in Fig. 3.11 and 3.12 . For high values of mass flux, the curves in Fig. 3.17 and 3.18 are steep, providing poor control, whereas for small values of mass flux, the curves become nearly linear with a small slope. Thus the two modes of operation, steady and pulsating fields, complement each other to provide an overall wide turndown ratio for practical operation.

For the experiments reported in Fig. 3.18, the frequency of the applied field was limited by the overall circuit. The field was pulsed by applying inhibiting pulses to the low voltage input of the EHT unit, so that the capacitor of the EHT had to be discharged through the material when the field was cut off and recharged for the next pulse. Thus the overall circuit response results from the EHT unit charging and discharging through the material and from particle movement around the electrodes, with the frequency limited by the capacitor discharge.

The electrical properties of the materials tested are given in Table 3.2, measured between the electrodes in the rectangular duct using a Wayne Kerr electrical bridge. The salt had a much shorter discharge time than sand or turnip seeds due to its lower resistivity, so that higher frequencies could 
be used with this material.

\section{Time Constant of EVS System for Voltage Step Change}

The time constants of the EVS for a step change in electric field were determined by measuring the flowrate from the EVS with a weighing device. The test rig shown in Fig. 3.9 was used for dispensing $\mathrm{NaCl}$ salt $(90-600 \mu \mathrm{m}$ diameter) from the $64 \mathrm{~mm}$ column with the electrode configuration shown in Fig. 1.1. The gap between the electrodes was $20 \mathrm{~mm}$ and the downstream electrode mesh size was $5 \mathrm{~mm}$.

For this work, two different weighing devices with similarly high performance specifications were available for measuring the flowrate response of the EVS. One was a tension load cell model TRFP-0100K-111, manufactured by Industrial Transducers Limited, with $100 \mathrm{~kg}$ capacity and a continuous analog output which was digitised by a fast 16 bit A/D converter at the computer interface. The load cell is physically designed to weigh objects which are suspended from it. The second weighing device was a weighing platform model F150S, manufactured by Sartorius Limited, with 150 $\mathrm{kg}$ capacity and a maximum digitised signal output frequency of $10 \mathrm{~Hz}$.

The overall system response consists of the combined responses of the EHT, the weighing device, charging and discharging of the material, and transportation time of the material from the electrodes to the collecting bin where it is weighed. The charge relaxation time of the particles is typically in the order of milliseconds, and the transportation time of the material can be easily varied. For the experimental setup used here, where the height between the valve and the bottom of the collection bin is about $1.0 \mathrm{~m}$, the transportation time has a minimum value of about $0.4 \mathrm{~s}$, as estimated by the free fall of the particles. Therefore, it remains to determine the response times of the EVS and the weighing device. To isolate the response characteristics of both the load cell and weighing platform from the response of the EVS, some simple measurements were first made without involving perturbations to the EVS. The weighing devices were then used to measure the flowrate response of the EVS to voltage step changes and to a PID control action for setpoint control.

Two types of tests were carried out on the weighing devices to assess their effectiveness for continuous flowrate monitoring. In the first test, each 
device was loaded with a single $1 \mathrm{~kg}$ weight and the response with time was measured. In the second test, the variability or 'noise' in the flowrate measurements of each device was determined for varying sampling intervals.

The results of the first test are shown in Fig. 3.19. For the weighing platform, the test was performed by dropping a $1 \mathrm{~kg}$ weight from a few centimetres height onto the platform. The maximum sampling rate of $10 \mathrm{~Hz}$ was used for recording the response of the weighing platform on the Sinclair computer. For the load cell, the weight was suspended about $30 \mathrm{~cm}$ below it with a stiff rope and then supported by hand before being let to fall through a few centimetres to its full extension. The continuous analog output of the load cell was recorded using a digital storage oscilloscope. The results in Fig. 3.19 show relatively long stabilisation times, compared to the maximum sampling frequencies available, and large overshoot in the readings. For the weighing platform, the test was repeated five times and in four of the runs, the readings showed overshoot of the 'true' weight before reaching the final correct value, with the worst case showing almost 20 per cent overshoot. The times required for the response to stabilise to the final correct weight were 0.2 to $0.4 \mathrm{~s}$. The response of the load cell in Fig. 3.19 shows oscillating behaviour with a maximum overshoot of about 140 per cent. The stabilisation time of the load cell was about 0.2 s. Quantitatively, the responses shown in Fig. 3.19 are certainly specific to the rather rough method of applying the weight. However, qualitatively, the overshoot effect is likely to exist to some extent whenever there is a step change in the applied weight, as when a change is made in the flowrate of granular material.

Fig. 3.20 shows the results of the noise tests. At each sampling interval, twenty flowrate readings were obtained from which the average and the relative standard deviation were determined. The flowrate was calculated from the change in accumulated weight over the sampling interval. Fig. 3.20 shows that for both weighing devices, it is necessary to use a sampling interval of about $1.0 \mathrm{~s}$ to reduce the relative standard deviation to 10 per cent. Therefore, although both devices are capable of much faster output frequencies, for reliable measurements in this testing rig, it is not advisable to use frequencies greater than about $1 \mathrm{~Hz}$. It is obvious that this response is the combined response of the weighing device and the transportation time. 
In conclusion, both weighing devices are capable of fast sampling rates. However due to their relatively long stablisation times, these sampling rates can only be taken advantage of at the expense of accuracy and precision. Therefore, these devices are suitable for measuring steady flowrates with slow fluctuations where slow sampling rates are tolerable, but they are essentially unsuitable for monitoring processes where fast transients are involved.

For the reasons outlined above, the measurement of the response characteristics of the EVS was essentially limited by the response of the weighing device. The results given above showed a large degree of variability in flowrate measurements made at frequencies greater than $1 \mathrm{~Hz}$. This is too slow for assessing the capability of the Evs for fast flow control. An alternative sampling procedure was also used to improve the precision of the readings. This involved measuring and recording the accumulated mass, rather than the flowrate, as shown in Fig. 3.21(a) and $3.21(b)$ for the load cell and the weighing platform, respectively. In this way, the signal processing involved in calculating the flowrate was eliminated, and a frequency of $5 \mathrm{~Hz}$ was used to record the response of the weighing devices to a step change in the voltage applied to the EVS. In Fig. 3.21, the flowrate through the EVS is represented by the slope of the line and therefore, a change in the flowrate in response to a change in the voltage is shown by a change in the slope.

Before and after a voltage step change occurs, the points on both curves in Fig. 3.21 follow closely straight lines, indicating little fluctuation from steady-state. In Fig. 3.21(a), the voltage on the EVS was decreased from 7 to $3 \mathrm{kV}$ at the time of $4.0 \mathrm{~s}$. The straight lines, which represent the flowrates before and after the step change, were calculated from two separate best linear fits to the data points from 0.0 to $3.8 \mathrm{~s}$ and from 5.2 to $6.4 \mathrm{~s}$. In Fig. 3.21(b), the voltage was increased from 0 to $6.5 \mathrm{kV}$ at the time of $1.0 \mathrm{~s}$, and the straight lines were calculated from two separate best linear fits to the data points from 0.0 to $0.8 \mathrm{~s}$ and from 2.0 to 3.2 s. From Fig. 3.21, the total time taken from the initiation of the voltage step change to the point when a new steady-state flowrate was attained was approximately 0.6 to $0.8 \mathrm{~s}$ for both cases. This result is in agreement with previous measurements, since the response of the system is dominated by the response time of the weighing device, about $0.2 \mathrm{~s}$, and the transportation time of the material, about $0.4 \mathrm{~s}$. Therefore, no additional 
precision was gained from this method of analysis, as compared to the previous method where actual flowrates were calculated. Finally, in Fig. 3.21(b), it is worth noting the overshoot followed by an apparent 'loss in weight' shown by the response of the weighing platform to the voltage increase.

Similar tests were carried out in an attempt to decouple the transportation time and the weighing device response time using a high speed ultraviolet chart recorder to continuously monitor the analog output of the load cell. In these tests, two voltage steps of different magnitudes were applied to the EVS, with each step being applied both as a voltage increase and a voltage decrease. As in Fig. 3.21, the response times of the system to these steps were determined from the changes in slope of the weight versus time chart traces. The results are shown in Table 3.3, where transportation time is defined as the time elapsed between the initiation of the voltage step and the first indication of a change in flowrate, and the weighing time is defined as the time elapsed from the start of the change in flowrate to the point when the new steady-state flowrate is attained.

In Table 3.3, the transportation times range from 0.3 to $0.5 \mathrm{~s}$, agreeing with the estimated time of $0.4 \mathrm{~s}$ for the salt to fall from the valve to the bottom of the bin. For the two voltage decreases of different magnitude, the weighing times can be considered to be essentially equal within the error of determining the actual points where slope changes start and end from the chart traces, and within the natural variation expected in normal operation. However, Table 3.3 shows that the weighing times for the two voltage increases differ significantly from one another, with the response time being larger for the step of larger magnitude. Also the weighing times for the voltage increases are much greater than for the voltage decreases. A possible cause for this behaviour is thought to be the overshoot in the voltage. By monitoring the potential of the upstream electrade using a digital storage oscilloscope, it has been observed that when the voltage is increased, it initially overshoots the required value. Therefore, the flowrate is essentially affected by two changes in voltage, rather than one, and this additional perturbation to the flow may affect the time required to reach steady-state. The results in Table 3.3 confirm that the system response time is dominated by the response of the weighing devices which makes it impossible to evaluate the time constant of the EVS 
with this method.

PID Control with EVS

The potential of the EVS for flow control was examined using a simple PID algorithm operated from a Sinclair QL computer which was interfaced with the EHT for controlling the voltage [53]. The load cell was used as the flowrate measuring device and initially, a frequency of $1 \mathrm{~Hz}$ was used for the flowrate sampling rate. However, it became immediately evident that while $1 \mathrm{~Hz}$ was sufficiently slow to produce stable measurements for steady-state flow, an unacceptable amount of instability was produced in the case of transient flows produced by controlling to a setpoint. It was therefore necessary to base the PID control on a moving average flowrate calculated from five flowrate measurements at one second intervals. Using a moving average, to represent the 'instantaneous' flowrate, slowed down the system response significantly. For example, for a voltage step change from 7 to $3 \mathrm{kV}$, the transportation time remained about $0.5 \mathrm{~s}$, but the effective weighing time was increased to $5 \mathrm{~s}$. However, using the moving average method at least allowed the response limitations of the load cell to be overcome in such a way that the control abilities of the Evs could be observed.

The response of the EVS to three changes in flowrate setpoint using a constant gain of $0.0678 \mathrm{~kg} \mathrm{~s}^{-1} \mathrm{kV}^{-1}$ is shown in Fig. 3.22. While the response is slow, the final setpoint error is negligible. For effective control, a PID method with constant gain is really only suited to linear processes. As shown by the EVS performance curve for $\mathrm{NaCl}$ salt in Fig. 3.23, this process is not linear over the full flowrate range. However, by taking a limited flowrate range for control with constant gain, the nonlinearity is small and acceptable control is obtained. The gain used for control in Fig. 3.22 was determined by trial and error tuning for the flowrate range of 0.15 to $0.45 \mathrm{~kg} \mathrm{~s}^{-1}$. In Fig. 3.23, the setpoint flowrate of $0.2 \mathrm{~kg} \mathrm{~s}^{-1}$ lies on the steep part of this range, where the flowrate is very sensitive to any change in voltage. Therefore the response of the EVS in Fig. 3.22 for the setpoint of $0.2 \mathrm{~kg} \mathrm{~s}^{-1}$ shows significant oscillation. The setpoints of 0.3 and $0.4 \mathrm{~kg} \mathrm{~s}^{-1}$ move progressively onto the flatter part of the curve in Fig. 3.23, so that the oscillating response of the EVS to these setpoints becomes damped in Fig. 3.22. Therefore, an obvious improvement to this control method would be the introduction of a self-tuning gain which is updated according to the flowrate response in the 
range of the setpoint.

\subsection{CONCLUSIONS}

The arching and the steady-state flow experiments show that the electrical clamping forces acting at individual particles generate significant net stresses in the bulk material such that gravitational forces can be counteracted and the flow of material halted. The shear cell experiments show that the shear stress required for failure at a particular value of applied mechanical load is increased by the electric field. The effect of the electric field on the yield shear stresses can be described either as producing an applied external stress or interparticle cohesion. The ability for granular material to form stable stress-free surfaces by arching in the wedge-shaped hopper and the EVS indicates that cohesion forces exist between the particles at the surface. Therefore from the experimental evidence alone, it is possible to interpret the qualitative effect of the electrical clamping forces as either an applied force or interparticle cohesion. This issue is discussed further in Chapter 4 where the experimental data is analysed using the single contact models of the electrical clamping force.

The evaluation of the steady-state flowrate in various configurations of the EVS shows that the flowrate depends on the average electric field strength and on the distribution of the electric field between the electrodes. Using a steady applied electric field, the EVS can be used successfully to halt the flow of a granular material and to control the flowrate in the upper half of the full flowrate range. A quantitative analysis of the steady-state flow is presented in Chapter 4.

The flow response of the EVS to changing electric fields was examined. The flow behaviour for pulsating fields with variable pulse width and frequency shows an opposite trend to the flow response for a steady field. In the pulsating mode, good control of flowrate is obtained in the lower half of the full flowrate range. Therefore, the two modes of operation complement each other to provide an overall wide turndown ratio.

Attempts to measure the time constant of the EVS for a voltage step change were unsuccessful because the response of the system was dominated by the 
response time of the weighing device which was used to monitor the change in flowrate. Due to the long stabilisation time of the weighing device, it was also impossible to exploit the fast response time of the EVS for controlling flow with a PID control programme. 


\section{CHAPTER 4}

\section{ANALYSIS OF MACROSCOPIC BULK BEHAVIOUR OF PARTICULATE SOLIDS WITH ELECTRIC FIELDS}

\subsection{INTRODUCTION}

When an electric field is applied to a packed bed of particulate solids, several types of electrical force may be generated, depending on the bulk resistivity of the solids and the geometry of the electrodes. For the electrode configurations and materials used in this work, these are notably the electrostatic attractive force, the polarization force, and the electrical clamping force.

The electrostatic attractive force acts between charged bodies. For the case of a granular material packed between two electrodes, this force is commonly obtained by treating the solids as a homogeneous dielectric continuum, and is hence given by [54]:

$$
\mathrm{F}_{\mathrm{es}} / \mathrm{A}=\varepsilon_{\mathrm{o}} \varepsilon_{\mathrm{s}} \mathrm{E}_{\mathrm{a}}^{2} / 2
$$

where $F_{\text {es }}$ is the compressive force exerted by the electrodes over an area $A, \varepsilon_{0}$ is the absolute permittivity of free space, $\varepsilon_{s}$ is the bulk relative permittivity (i.e. dielectric constant of the solids), and $\mathrm{E}_{\mathrm{a}}$ is the average electric field. According to equation (4.1), the electrostatic attractive force in the wall shear experiments presented in Chapter 3 is obtained by considering that the two plate electrodes were filled with a homogeneous dielectric material with a dielectric constant of about 7, i.e. that of glass [55]. For the electric field strengths of 390 and $790 \mathrm{kV} \mathrm{m}^{-1}$ which were used in the wall shear experiments, the resulting electrostatic attractive stresses are approximately 5 and $20 \mathrm{~N} \mathrm{~m}^{-2}$, respectively. These stresses are too small compared to the values of electrical normal stress, $\sigma_{e}$, shown in Table 3.1 which were obtained from the experimental data.

The polarization force is generated when uncharged particles become polarized in a non-uniform electric field [14]. A formulation for the polarization force for a dielectric sphere touching a conductive plate has been developed by Dietz [56] as 


$$
f_{p}=4 \pi \varepsilon_{f} R^{2} E_{a}^{2} \zeta\left(\frac{3 \eta^{2}}{2(3-\eta)^{2}(1-\eta)}\right)
$$

where $\eta=\frac{\varepsilon_{p}-\varepsilon_{f}}{\varepsilon_{p}+\varepsilon_{f}}$

In equation (4.2), $\varepsilon_{p}$ and $\varepsilon_{f}$ are the absolute permittivities of the particles and the interstitial fluid respectively. The value of $\zeta$ is dependent on the value of $\eta$ and is found from a numerical solution given by Dietz [56]. For glass with a relative permittivity of approximately 7.0 (with respect to the permittivity of free space) and air with a relative permittivity of approximately $1, \eta$ has a value of 0.75 and $\zeta$ has a value of approximately 1.18. Using an average radius of $330 \mu \mathrm{m}$ for the $600-710 \mu \mathrm{m}$ particles used in the wall shear experiments from Chapter 3, the polarization force on each particle at the wall electrode is 1.5 and $6.0 \mu \mathrm{N}$ for fields of 390 and $790 \mathrm{kV} \cdot \mathrm{m}^{-1}$, respectively. The average normal stress on the wall, $\sigma_{p}$, is obtained from the individual particle forces by assuming a spacing of approximately two times the particle radius between particle centres such that

$$
\sigma_{p}=f_{p} /(2 \mathrm{R})^{2}
$$

Therefore, the normal stresses generated by polarization forces at the wall are approximately 3 and $14 \mathrm{~N} \mathrm{~m}^{-2}$ for fields of 390 and $790 \mathrm{kV} \mathrm{m}^{-1}$, respectively. As for the electrostatic attractive stresses, the polarization stresses are too small to increase the shear stresses by the amounts shown experimentally in Chapter 3.

The third electrical force which acts on a packed bed of particles in an electric field is the electrical clamping force. As discussed in Chapter 1, this force acts at the contact points between particles, and between particles and electrodes, by the electric current flowing through the bed. The forces are generated by the constriction of electric current at the contact points, where the local electric field across the interparticle gap is greatly enhanced. Provided that the particles are not too conductive so as to draw excessively high currents, nor too resistive so as to draw such low currents that there is insufficient effect, the forces generated may be sufficiently large and comparable in magnitude with external forces, such as those due to gravity, so that the bulk failure characteristics of the solids are substantially altered. As reviewed in previous chapters, the 
adhesion of the deposited dust layer in electrostatic precipitators, the pressure drop overshoot in electrofluidised beds, and the operation of the Electromechanical Valve for Solids are all considered to be caused by the presence of electrical clamping forces.

Several research groups have modelled the electrical clamping force. Dietz [19], Dietz and Melcher [20,39], Moslehi and Self [16] and McLean [15] all consider spherical geometries. They analyse the effect of electrical clamping forces on the net stress state of particulate assemblies using a force balance approach. Relatively recently, Kendall [31] has developed another model for the electrical clamping force in a cylindrical geometry where the behaviour of the bodies under electrical and mechanical forces is analysed using an energy balance approach. Kendall's method is reviewed in Chapter 5 where it is also shown that his model for a cylindrical geometry cannot easily be applied to the spherical geometries of interparticle contacts treated in this work. Therefore in this chapter, the former category of models referred to above will be considered in the evaluation of bulk behaviour.

There are several theoretical models of the electrical clamping force [15,16,19]. These models differ in details, depending on the mechanical properties of the contact points, and on the limit of electric field breakdown in the interparticle gap. However, they all relate the clamping force to the average applied electric field strength, $E_{a}$, for a single contact between two spheres, or between a sphere and a conductive plane, and can be represented by:

$$
f_{e}=\alpha E_{a}^{\gamma}
$$

where $f_{e}$ is the interparticle electrical clamping force, $\alpha$ is a function of the physical and electrical properties of the particles, and $\gamma$ is the power law constant.

In general, three types of contact characteristics are considered in the models. The value of $\gamma$ in equation (4.4) depends on these characteristics. In the first case, the contact cap size is considered to be determined only by the local mechanical load and is given by the Hertz analysis [57,58]:

$$
a=\left(\frac{3 \pi F}{8 R_{\text {eff }} \bar{E}_{\text {eff }}}\right)^{1 / 3}
$$


where $a$ is the contact cap radius, $F$ is the normal force at the contact, and $R_{\text {eff }}$ and $E_{\text {eff }}$ are the radius of curvature and effective Young's modulus, respectively, as given in the Nomenclature. Since the contact cap size is independent of the electric field in this analysis, the resulting model for the electrical clamping force is referred to as the Fixed Cap model. For the Fixed Cap model where the size of the contact cap is independent of the electric field strength, $\gamma$ can be analytically calculated and is equal to $2[15,16,19]$.

The Fixed Cap model neglects any contact deformation which might occur due to the electrical clamping force itself acting at the contact. On the other hand, the second case of electrical clamping force models assumes that the size of the contact cap is influenced only by the electrical clamping force with no contribution from any mechanical loading at the contact. The cap size in this case is again given by the Hertz analysis in equation (4.5). In this analysis the contact cap deforms elastically as a function of the electric field. The resulting model for the electrical clamping force is referred to as the Elastic Cap model. For the Elastic Cap model the value of $\gamma$ depends on the mathematical method used to solve the equations. The value of $\gamma$ falls in the range of 1.2 to $1.5[15,16,19]$.

The third case of electrical clamping force models assumes that conditions at the contact are such that a sufficiently high local electric field is produced to cause electrical breakdown of the interstitial gas. In this case, the electric field which exists in the gap outside the contact is limited by the electrical breakdown field strength of the gas. This condition may be relevant if the size of the contact point is very small, such as for very fine particles, or for particles with sharp asperities. The force model developed based on this electrical contact behaviour is referred to as the Limited Field model. For the Limited Field model, the value of $\gamma$ is approximately unity [19].

The development of the equations for the Fixed Cap, Elastic Cap and Limited Field models is outlined in Appendix 4.1. Of the three research groups who modelled the electrical clamping forces for spherical geometries using a force balance approach, the analytical methods of Dietz [19] and Moslehi and Self [16] are the most rigorous. Dietz [19] developed equations for all three cases of Fixed Cap, Elastic Cap, and Limited Field assuming 
surface conduction. The final equations for these models are given in Appendix 4.1.

Moslehi and Self [16] considered both surface and volume conduction in their formulations of the Fixed Cap and Elastic Cap models. They did not develop an equation for the Limited Field model. The final equations of Moslehi and Self for the electrical clamping force models are given in Appendix 4.1. In Appendix 4.1, it is also shown that the volume conduction models of Moslehi and Self predict larger values for the electrical clamping force than the surface conduction models. For the dielectric materials used in the experiments in Chapter 3, i.e. glass and sand, at ambient temperature conditions, surface conduction dominates over volume conduction [16].

In Appendix 4.1, it is shown that the surface conduction models of Dietz [19] and Moslehi and Self [16] are similar for the Fixed Cap and Elastic Cap cases. Therefore, in the analyses presented below in this chapter only the Fixed Cap and Elastic Cap models of Moslehi and Self have been used. The models of Moslehi and Self were chosen because they appear to be more self-consistent than those of Dietz. This point is shown in Appendix 4.1. There, the Elastic Cap model is used to calculate a force, $f$, for a particular field strength. This value of $f$ is then used in the Hertz model equation (4.5) to calculate a value of the contact cap, a. If this value of contact cap, $a$, is then used in the Fixed Cap model to calculate a force, the resulting force should be the same as that calculated by the Elastic Cap model, f. For Moslehi and Self's models this is true, but for Dietz' models it is not. For this reason, the Fixed Cap and Elastic Cap models of Moslehi and Self for surface conduction have been used in the analyses. Since Moslehi and Self did not develop a Limited Field model, the Limited Field model of Dietz [19] has been used for this case.

There has so far been no attempt to measure the electrical clamping force for a single contact point, and hence the verification of the above models has been inferred from measurements of bulk behaviour and properties, such as the pressure drop overshoot in electrofluidised beds [39], or the tensile force required to detach an electrode from a bed of particulate solids [15]. However, it is necessary for the analysis of experimental data to consider how the electrical clamping forces, which are microscopic by nature, can be translated into a macroscopic representation. This 
transformation is considered below for the experimental results presented in Chapter 3.

\subsection{TRANSFORMATION OF MICROSCOPIC FORCE MODELS TO MACROSCOPIC REPRESENTATION}

One approach to modelling the electromechanics of assemblages of particles is by assembly calculations following the approach of Thornton [59] and Walton and Braun [60]. There, the microcontact frictional behaviour of particles may be modified to include an additional normal load, $f_{e}$, due to the electrical clamping force so that the total normal load at each contact is given by $f_{t}=f_{e}+f_{m}$, where $f_{m}$ is the contact force due to prevailing mechanical stresses. It is possible to incorporate this approach into both elastic and elastic/plastic analyses of contact frictional behaviour as demonstrated by Kendall [61] in dealing with Van der Waals forces observed in fine powders.

However, unlike the short range (cohesive) forces which result from intrinsic material properties, the interparticle forces induced by the action of an applied electric field will have directional nature. This is because contacts between surfaces on equipotential lines do not generate electrical clamping forces. Therefore, prior to assembly calculations to generate internal stress distributions, it is necessary to calculate the current paths and the prevailing local electric fields at contact points. Two examples of possible contact arrangements are shown in Fig. 4.1, indicating the complexity of such analyses. For each contact arrangement, the corresponding circuit diagram is also shown in terms of the resistances of the current paths. By comparing the circuit diagrams of the two contact arrangements, it can be shown that although both arrangements have contacts in the direction perpendicular to the field, in the case of Fig. 4.1(c) there will be an electrical clamping force perpendicular to the electric field, while in the case of Fig. 4.1(a) there will not. In Fig. 4.1(a), the current will flow through both particle strings. Since these strings are paths of equal resistance, the voltage on either side of the single central particle will be the same. Because there is no potential difference across these horizontal contacts, there will be no local electric fields generated at these contacts and therefore no electrical clamping forces. In Fig. 4.1(c), the horizontal contact is in series with the particle string which forms the current path, rather than in parallel 
as in Fig. 4.1(a). Therefore, in this case there will be a voltage difference across the two particles bounding the horizontal contact. A local electric field will exist in the contact gap and therefore, in this assembly there will be an electrical clamping force perpendicular to the direction of the overall applied electric field.

From the analysis of the two simple particle assemblies in Fig. 4.1 , it is clear that the calculation of the electrical clamping forces at individual contacts in a randomly packed assembly is very complicated and has a pattern which is different from the distribution of mechanical loads. However in general, since the net flow of electric current will be in the direction of the overall applied field, the maximum net electrical clamping stress will also be in the direction of the field, and since there will be only a small if not zero net current flow in the direction perpendicular to the field, there will be only a small if not zero net electrical clamping stress perpendicular to the field.

For wall/particle contacts, the randomness of the current paths is avoided so that a relatively simple analysis is possible to calculate the wall stress profile from a microcontact mechanical model by incorporating the effects of local bed porosity and the state of bulk failure [62]. This analysis will be addressed below as it provides some insight into the nature of the electrical clamping forces, and will also provide a possible means to verify the present electrical clamping force models.

Alternatively to assembly calculations, a more common approach for modelling the behaviour of assemblies of particles uses continuum mechanics theories, where the criterion for plastic yield is given by the tangency of the Mohr's stress circle and the yield locus [46]. The question then arises as to whether the electrical clamping forces should be incorporated into the yield locus, by modifying the cohesive/adhesive properties of the material, or into the stress state represented by the Mohr's circle. It is complicated to incorporate the electrical clamping forces into the yield locus, because the yield locus no longer has a simple functional relationship which can be applied isotropically to the material, due to the directionality of the electrical clamping forces. The failure here becomes more akin to that of anisotropic crystalline materials where the failure occurs on certain energetically favoured planes. A rigorous analysis using this approach becomes increasingly difficult for analytical approaches, and 
is more suitable for numerical methods which can take account of anisotropy. It is therefore mathematically and conceptually more convenient to include the electrical clamping forces into the stress state given by the Mohr's circle in the first instance, as will be shown in Sections 4.3 and 4.4. The former method, which considers the possibility of electrical cohesion, is also addressed later in Section 4.4.

Due to the complications of modifying the yield criterion, the analysis and interpretation of experimental data on electrical clamping forces in the literature have essentially adopted the latter approach given above of modifying the stress state, albeit referring to the phenomenon as electrical cohesion [15,32,39]. A similar approach has also been adopted in the analysis of the mechanics of magnetic powders under a magnetic field [63]. The underlying assumption here is that the mobilisation of shear stresses is based purely on the mechanical properties of the material, and that the yield locus remains invariant with the direction of the applied electric field. This is a key assumption which has not so far been verified. In addressing this question, it is necessary to consider whether the electrical clamping forces are of the ponderomotive type, or of an internal nature. The former should generate an external compressive stress between the electrodes beyond that prevailing due to the electrostatic attraction between the electrodes in equation (4.1), thus acting in addition to the normal mechanical load. For the latter case, these forces are purely interparticle so that the net external force acting on the packed bed is zero. Such a system should exhibit resistance to shear even in the absence of a mechanical load, but without any tendency to consolidate. The currently available experimental data in the literature do not allow the elucidation of the appropriate alternative because the action of the electrical clamping forces can be interpreted in either way due to the limitation of the experimental design.

The choice of incorporating the electrical clamping forces in the yield locus or Mohr's circle is more than purely a matter of representation, and has implications on application, e.g. in the interpretation of the mechanism of operation of the EVS. The operation of the EVS is by formation of stable compressive or tensile arches [64], depending on the electrode configuration, whose stability is brought about purely by electrical forces in the case of large and cohesionless particles. By analogy with cohesive arching of fine powders, the incorporation of 
electrical clamping forces in the yield locus implies the presence of an unconfined yield stress produced by electrical clamping forces, which is responsible for a stress free surface. In contrast, where the electrical clamping forces are incorporated in the stress state, there is a difficulty in explaining the presence of stable free surfaces for cohesionless materials without recourse to other electrical phenomena such as dielectrophoresis [52]. These issues are addressed below in the analysis of shear stresses at a wall and internally, and in the analysis of arching with electric fields.

\subsection{INTERNAL AND WALL SHEAR STRESSES}

It is apparent from the above discussion that the analysis of wall friction in the presence of electrical clamping forces is more tractable than that of internal friction in granular media. In the case of internal failure, the size and position of the shear zone is not well defined, whereas in the case of wall friction all the particle/wall contacts lie on a single plane. This facilitates the translation of the microscopic electrical clamping forces, predicted from theory, to macroscopic representation, because reasonable assumptions can be made about particle packing at the wall. Therefore, the case of yield at a wall is considered first, followed by the analysis of internal yield.

\section{Analysis of Wall Shear Stresses}

In Chapter 3, the experimental data of Robinson and Jones [32] for the yield of glass ballotini over a brass plate electrode shows that the electric field causes a shift in the yield loci approximately parallel to the zero field locus, thus suggesting that the angle of wall friction remains unchanged. On this basis, Robinson and Jones suggested that the electrical stress acts as an external load in addition to the normal mechanical load, and proceeded with the formulation of the shear stress given by

$$
\tau=\mu\left(\sigma_{m}+\sigma_{e}\right)+c
$$

where $\tau$ is the yield shear stress, $\sigma_{m}$ is the normal mechanical stress due to the weights on the bed of glass ballotini, $\sigma_{e}$ is the electrical stress, 
$\mu$ is the friction coefficient, and $c$ is the natural adhesion of the material in the absence of an electric field. For the glass ballotini used here, $c$ is essentially zero. The electrical stresses obtained from a best fit analysis of the data to equation (4.6) are given in Table 3.1 as 72 and $203 \mathrm{~N} \mathrm{~m}^{-2}$ for electric fields of 390 and $790 \mathrm{kV} \mathrm{m}^{-1}$, respectively. As shown above, the electrostatic attractive stresses given by equation (4.1) and the polarization stresses given by equations (4.2) and (4.3) are too small compared to the electrical normal stresses fitted from the experimental data. While Robinson and Jones attributed the generation of the electrical stress to the electrical clamping forces, no attempt was made to relate the electrical stress to the single contact models of the electrical clamping force.

In the following analysis, the single contact models of the electrical clamping force are used to estimate the electrical stress. Using equation (4.4) for the general form of the electrical clamping force, the net electrical clamping stress, $\sigma_{\mathrm{e}}$, is simply given by

$$
\sigma_{\mathrm{e}}=\frac{\alpha \mathrm{E}_{\mathrm{a}}^{\gamma}}{\mathrm{s}^{2}}
$$

where $\mathrm{S}$ is the average distance between the particle centres at the wall. Direct observations as well as measurements of local transient stresses transmitted to the walls suggest that, for regular close packed assemblies of spheres, the separation distance $S$ between particle centres is roughly equal to 2 particle radii [62]. Therefore, the value of $S$ is assumed to equal $2 \mathrm{R}$ in the following analysis.

Four types of particle/wall contact characteristics have been examined by using the different models of the electrical clamping force discussed above. The Fixed Cap, Elastic Cap, and Limited Field models are used, and in addition, a model is developed which takes into account the effect of both mechanical and electrical forces on the contact size. The two forces are added together to form the new total load $F$ in equation (4.5) in the calculation of the cap radius, a. The Fixed Cap model is then used to calculate the resulting electrical clamping force. As the electrical clamping force is inversely proportional to the cap size, an iterative procedure is necessary to calculate the equilibrium cap size and electrical clamping force. This will be referred to as the Combined Force model. The 
equations for the Fixed Cap, Elastic Cap, and Limited Field models are given in Appendix 4.1 as discussed above.

The electrical clamping stress has been calculated for the wall yield loci in Fig. 3.3 using each of the four models according to equation (4.7). In all the models, conduction is assumed to occur over the particle surfaces only, with no contribution from the volume. This is normally the case for dielectric materials, where surface conduction dominates at ambient conditions. The results of the calculations for the Fixed Cap, Elastic Cap and Combined Force models with mechanical normal stresses of 200 and 600 $\mathrm{N} \mathrm{m}^{-2}$ are shown in Table 4.1. In Fig. 4.2, the best fit lines for the experimental yield loci at zero and $390 \mathrm{kV} \mathrm{m}^{-1}$ fields are compared with the theoretical predictions using values of $\sigma_{e}$ from equation (4.7) in equation (4.6). The value of $\sigma_{e}$ calculated from the Limited Field model is so small that the Limited Field line is essentially coincident with the zero field locus, and therefore it has not been shown in Fig. 4.2.

Two significant differences between theory and experiment are apparent from Fig. 4.2 and Table 4.1. First, the contribution of the electrical force is significantly overpredicted, by two orders of magnitude by the first three contact cap models, while the results of the Limited Field model produce negligible shift from the zero field strength. Second, the magnitude of the stress at incipient yield is now shown to decrease with increasing normal mechanical load acting on the plate for the Fixed Cap model. This response is clearly unrealistic and has resulted from the sensitivity of the electrical clamping force to the contact cap size as determined solely from the mechanical normal stress. Because the normal stress increases from 200 to $600 \mathrm{~N} \mathrm{~m}^{-2}$, the contact cap radius increases as shown in Table 4.1. Consequently the electrical clamping force decreases so that the contribution of $\sigma_{e}$ in equation (4.6) is a non-linear function of $\sigma_{m}$. This clearly shows that it is not correct to ignore the contribution of the electrical clamping forces to the deformation of the contact region.

The. results of the Elastic Cap model and the Combined Force model shown in Fig. 4.2 and Table 4.1 are very similar. Here, the Elastic Cap model of Moslehi and Self [16] with $\gamma$ equal to 1.2 has been used with the modification to allow the analysis of a sphere/wall contact instead of a sphere/sphere contact (see Appendix 4.1). From Table 4.1, it can be seen that the electrical clamping stresses predicted by the Elastic Cap and 
Combined Force models are smaller than those predicted by the Fixed Cap model because the magnitude of the electrical clamping forces are such that they produce greater contact deformation than the mechanical forces. As the contact cap size increases, the electrical resistance of the contact decreases and thus the electrical clamping force decreases. The electrical stresses predicted by the Elastic Cap model are independent of the mechanical stresses and thus the yield locus predicted from this model is a straight line in Fig. 4.2 .

The inclusion of the mechanical force with the electrical force in determining the cap size in the Combined Force model results in predictions of the electrical clamping forces which are only very slightly smaller than those predicted by the Elastic Cap model, which also indicates dominance of electrically caused deformation over mechanically caused deformation. Because the deformation caused by the electrical clamping forces dominates significantly over the deformation caused by the mechanical forces, there is only a very slight decrease in the electrical clamping stress predicted by the Combined Force model when the mechanical stress acting on the particles is increased from 200 to $600 \mathrm{~N} \mathrm{~m}^{-2}$. Table 4.1 shows that the electrical stresses predicted by the Combined Force model at the two field strengths of 390 and $790 \mathrm{kV} \mathrm{m}^{-1}$ are essentially constant with changing mechanical normal load. Thus the yield locus in Fig. 4.2 predicted from the Combined Force model is essentially a straight line. The experimental yield loci shown in Fig. 3.3 are in agreement with this behaviour since at both field strengths the yield loci are parallel with the zero field strength locus, which indicates that the mechanical forces are not influencing the electrical forces.

However, the actual increase in shear stress which is predicted by the Elastic Cap and Combined Force models differs significantly from the experimental measurements. Comparing the predictions of the models in Table 4.1 shows that the electrical clamping forces dominate greatly in magnitude over the mechanical forces. Much higher shear stresses should therefore be generated by the electrical clamping forces than by the mechanical normal forces. From Fig. 4.2 the increase in shear stress from zero field is about $530 \mathrm{~N} \mathrm{~m}^{-2}$ for the $390 \mathrm{kV} \mathrm{m}^{-1}$ field strength. This is entirely contradicted by the experimental evidence presented in Fig. 3.3, where the increase in shear stress from zero field is only about $18 \mathrm{~N} \mathrm{~m}^{-2}$ for the field strength $390 \mathrm{kV} \mathrm{m}^{-1}$. 
Finally, the results of the electrical clamping stress calculations using the Limited Field model of Dietz [19], where the value of $\gamma$ in the electrical clamping force equation is here 1.054, produce negligible shifts from the zero field case. The electrical clamping normal stresses for the 390 and $790 \mathrm{kV} \mathrm{m}{ }^{-1}$ electric fields are 5.2 and $11.0 \mathrm{~N} \mathrm{~m}^{-2}$ respectively and are therefore too small as compared to the experimental data. In the Limited Field model, the value of the electrical clamping force is approximately linearly dependent on the breakdown electric field strength of the interstitial gas. It is assumed that in the gap surrounding the contact cap, the electric field is limited to this value. In the calculations here, the breakdown strength of air, $3 \times 10^{6} \mathrm{~V} \mathrm{~m}^{-1}$, was assumed. The use of this value implies that the mechanism for breakdown is by gas discharge. However, for very small gaps, comparable with the mean free path of the gas molecules, the mechanism of electrical discharge under vacuum is more appropriate, where the breakdown field is typically greater than or equal to $10^{8} \mathrm{~V} \mathrm{~m}^{-1}$ [16]. Such high local electric fields could be achieved if the height of the gap surrounding the contact was very small, e.g. less than $1 \mu \mathrm{m}$, or for rough particles where microprojections on the surface could lead to significant field enhancement. However, a reliable estimate of the actual limiting field is difficult. The glass ballotini used here is relatively large $(600-710 \mu \mathrm{m})$ and smooth, so it is not expected that significant field breakdown would occur. Furthermore, the current-voltage relationship, as measured for this work, is purely of ohmic type and therefore the non-linear charge transport, thought to exist for fine powders [15], may not be applicable. Therefore, the Limited Field model is thought not to be pertinent to this case.

It is clear from the above analysis that there is a large discrepancy between the theoretical predictions and experimental measurements of wall shear under electric fields. The significance of this discrepancy is discussed in detail following the analysis of internal shear stresses below.

\section{Analysis of Internal Shear Stresses}

Compared to the case of particle/wall contacts, the calculation of the net electrical clamping stress on an internal plane within a granular material is complicated because of the directionality of the electrical clamping 
forces. The value of the electrical clamping force at an individual contact depends on the current path. It is straightforward to determine the current paths at a wall interface or in a regular assembly of particles. In a randomly packed assembly of particles, this calculation is complicated, However, by making simplifying assumptions that the probability of contacts between the particles is uniformly distributed over the surfaces, and that for contacts whose normal makes an angle $\psi$ with the direction of overall applied electric field the appropriate value of the local electric field strength is $E_{a} \cos \psi$, then a simple averaging can be performed in order to calculate the macroscopic stress from the single contact force. Thus, the average value of the electrical clamping force at a contact whose probability of position over a hemisphere is uniform, $\bar{f}_{e}$, resolved in the direction of the overall applied field can be calculated by integrating over the hemisphere such that

$$
\overline{\mathrm{f}}_{\mathrm{e}} 2 \pi \mathrm{R}^{2}=\oint \alpha\left(\mathrm{E}_{\mathrm{a}} \cos \psi\right)^{\gamma} \cos \psi d \mathrm{~A}_{\mathrm{h}}
$$

where $A_{h}$ is the surface of the hemisphere. The solution of equation (4.8) can be expressed in the following general form:

$$
\overline{\mathrm{f}}_{\mathrm{e}}=\frac{1}{v} \alpha \mathrm{E}_{\mathrm{a}}^{\gamma}
$$

where $v=\gamma+2$

From the value of $\overline{\mathrm{f}}_{\mathrm{e}}$, it is possible to obtain an average value for the internal electrical clamping stress, $\sigma_{\mathrm{e}}$, where

$$
\begin{aligned}
\sigma_{e} & =\left(\frac{\text { average force }}{\text { contact }}\right)\left(\frac{\text { number of contacts }}{\text { pair of particles }}\right)\left(\frac{\text { number of particles }}{\text { unit area }}\right) \\
& =\left(\frac{1}{v} \alpha \mathrm{E}_{\mathrm{a}}^{\gamma}\right)\left(\frac{\mathrm{N}}{2}\right)\left(\frac{1-\varepsilon}{\frac{1}{6} \pi \mathrm{d}_{\mathrm{p}}^{2}}\right)
\end{aligned}
$$

where $N$ is the coordination number, $\varepsilon$ is the voidage and $d_{p}$ is the particle diameter. In equation (4.10), it is assumed that the voidage on all planes is equal, and that the number of particles per unit area is given according to Rumpf [65] by $(1-\varepsilon) /\left(\frac{1}{6} \pi d_{p}^{2}\right)$.

In the following, the electrical stress calculated from equation (4.10) is compared with experimental data presented in Chapter 3 for the internal 
yield of glass ballotini in a box shear cell. Similarly to the wall yield loci of Robinson and Jones [32], the internal yield experiments showed that the electric field causes a shift in the internal yield loci approximately parallel to the zero field locus. As with the wall yield experiment, it can be considered that the electrical stress produced by the field acts as an external load in addition to the normal mechanical load. By fitting the experimental data from the internal yield experiment to equation (4.6), the electrical stresses are given in Table 3.1 as 385 and $703 \mathrm{~N} \mathrm{~m}^{-2}$ for electric field strengths of 470 and $800 \mathrm{kV} \mathrm{m}^{-1}$, respectively.

The theoretical predictions of the electrical clamping stress generated on an internal plane normal to the overall applied field can be calculated using equation (4.10). The coordination number, $\mathrm{N}$, is assumed to be 6 . The packed bulk density of the glass ballotini in the shear cell was measured to be $1500 \mathrm{~kg} \mathrm{~m}^{-3}$ and the particle density was $2500 \mathrm{~kg} \mathrm{~m}^{-3}$. Therefore, the voidage, $\varepsilon$, is 0.4 .

The electrical clamping stress calculations using the Elastic Cap and Combined Force models for the data of Robinson and Jones [32] showed that for their ranges of mechanical normal stresses and applied electric fields, the contribution of the mechanical force to the deformation of the contact cap had negligible effect on the value of the electrical clamping stress. The magnitudes of the applied fields used for the internal yield data are approximately the same as those of Robinson and Jones. However, a much higher range of mechanical normal stresses was used in the internal yield experiments, so the contribution of the mechanical forces here may be more significant.

The values of the electrical clamping stress from equation (4.10) with the Elastic Cap model for sphere/sphere contacts (see Appendix 4.1) are predicted to be 3.7 and $7.4 \mathrm{kN} \mathrm{m}^{-2}$ for the electric fields of 470 and 800 $\mathrm{kV} \mathrm{m}{ }^{-1}$, respectively. These values are about one order of magnitude larger than the experimental results.

The effect of the higher mechanical force range was checked by calculating the electrical clamping stress using the Combined Force model at the highest normal mechanical stress used in the internal yield experiments, $3.7 \mathrm{kN} \mathrm{m}^{-2}$. The average normal mechanical force per particle on the plane was obtained by dividing the stress by the number of particles per unit 
area according to the expression shown in equation (4.10). The resulting force per particle is $1.4 \mathrm{mN}$. In calculating the electrical clamping stress, the Combined Force model uses the equation for the Fixed Cap electrical clamping force model with both the mechanical and electrical force contributions to the contact cap radius, a. In order to obtain an estimate of the maximum possible effect of the mechanical loading on the electrical clamping stress, the full magnitude of the mechanical force per particle was assumed to act at each interparticle contact to determine the contact cap size. The values of the electrical clamping stress predicted in this way by the Combined Force model are 2.8 and $6.9 \mathrm{kN} \mathrm{m}^{-2}$ for field strengths of 470 and $800 \mathrm{kV} \mathrm{m}{ }^{-1}$, respectively. Comparing the values of $\sigma_{e}$ from the Combined Force model with those from the Elastic Cap model given above shows that for the normal mechanical load range and electric field strengths used in the internal yield experiments, the contribution of the mechanical forces to the contact cap size is still minor compared to the contribution of the electrical clamping forces, i.e. similar to the wall yield case.

The predictions of the electrical clamping stress from the Limited Field model are 7 and $13 \mathrm{~N} \mathrm{~m}^{-2}$ for the 470 and $800 \mathrm{kV} \mathrm{m}^{-1}$ field strengths respectively. As in the analysis of wall-particle adhesion, these values of electrical stress are negligible compared to the experimental values, and for the reasons discussed earlier, the Limited Field model is not considered pertinent to the particulate system used here.

If the electric field is generating additional external stresses on the material, it should be possible to determine the combined electromechanical stress state by superimposing the electrical stresses onto the mechanical stresses. This is conveniently done using Mohr's circle representations of the stress state according to Fig. 4.3.

In Fig. 4.3(a), the stresses due to the electric field have been represented by a Mohr's circle with the major principal stress $\sigma_{e 1}$, and minor principal stress $\sigma_{\mathrm{e} 2}$. As discussed earlier, an electric field is expected to produce the maximum electrical clamping force in the direction of the electric field and zero net force in the direction normal to the field. Therefore, the major principal stress, $\sigma_{\mathrm{e} 1}$, in Fig. 4.3(a) must be the maximum stress which is acting in the direction of the field, and the minor principal stress, $\sigma_{\mathrm{ez}}$, is zero in the direction normal to the field. 
The mechanical stress state and internal yield locus of a cohesionless material under application of a normal load, $w_{1}$, are shown in Fig. 4.3(b). In order to superimpose the two electrical and mechanical stress circles, the direction of the stresses in one system must be known relative to the stresses in the other system. For the experimental data presented in Chapter 3 for the internal yield of glass ballotini in a box shear cell, the electric field is applied in the same direction as the normal mechanical load, i.e. normal to the shear cell lid in the vertical direction. Therefore, the planes represented by points $\mathrm{A}_{e}$ and $\mathrm{B}_{e}$ on the electrical Mohr's circle must be the same planes as represented by points $A_{m}$ and $B_{m}$ on the mechanical Mohr's circle. By adding the corresponding electrical and mechanical stresses on the two planes, two points are obtained to describe the new Mohr's circle for the combined stress state. The new total normal and shear stresses acting on the plane normal to the electric field are therefore equal to $\sigma_{e 1}+w_{1}$ and $s_{1}$ respectively. On the plane parallel to the electric field, the new total stresses are $w_{2}$ and $-s_{1}$. The new combined Mohr's circle is shown in Fig. 4.3(c). Relative to the original mechanical circle, the new Mohr's circle has been shifted to the right and has changed size. The circle has also been rotated so that the directions of the major and minor principal stress planes have changed relative to the direction of the plane normal to the electric field.

Since it has been assumed that the yield locus remains unchanged, the position of the new circle relative to the yield locus will determine whether yielding stress conditions still exist in the material. The rotation, shift and radius of the new Mohr's circle can be determined from the two known points on the circle using simple geometrical relationships. By referring to Fig. $4.3(\mathrm{c})$, the values of the mean principal stress, $P_{t}$, radius, $R_{t}$, and angle of rotation, $\chi$, of the new Mohr's circle can be calculated using the following equations:

$$
\begin{aligned}
P_{t} & =\frac{w_{2}+w_{1}+\sigma_{e 1}}{2} \\
R_{t} & =\left(x_{t}^{2}+y_{t}^{2}\right)^{1 / 2} \\
= & {\left[0.25\left(w_{2}-w_{1}\right)^{2}-0.5 \sigma_{e 1}\left(w_{2}-w_{1}\right)+0.25 \sigma_{e 1}{ }^{2}+s_{1}{ }^{2}\right]^{1 / 2} } \\
2 x & =\sin ^{-1}\left(s_{1} / R_{t}\right)
\end{aligned}
$$


Upon application of an electrical normal stress, $\sigma_{e 1}$, the new condition of yielding will be described by the point of tangency of the new Mohr's circle and the yield locus, where

$$
R_{t}=P_{t} \sin \delta
$$

If the value of $R_{t}<P_{t} \sin \delta$, the material will no longer be in a state of yield. The solution of equation (4.14) using equations (4.11) and (4.12) is a quadratic equation with two solutions:

$$
\begin{aligned}
& \sigma_{e 1}=\frac{-b-\left(b^{2}-4 a c\right)^{1 / 2}}{2 a}=\sigma_{e 1 \min } \\
& \sigma_{e 1}=\frac{-b+\left(b^{2}-4 a c\right)^{1 / 2}}{2 a}=\sigma_{e 1 \max }
\end{aligned}
$$

where

$$
\begin{aligned}
& \mathrm{a}=0.25\left(1-\sin ^{2} \delta\right) \\
& \mathrm{b}=-0.5\left\{\left(\mathrm{w}_{2}-\mathrm{w}_{1}\right)+\left(\mathrm{w}_{2}+\mathrm{w}_{1}\right) \sin ^{2} \delta\right\} \\
& \mathrm{c}=0.25\left(\mathrm{w}_{2}-\mathrm{w}_{1}\right)^{2}-0.25\left(\mathrm{w}_{2}+\mathrm{w}_{1}\right)^{2} \sin ^{2} \delta+\mathrm{s}_{1}{ }^{2}
\end{aligned}
$$

Therefore, if an electric field is applied to a material initially in a state of yield, such that $\sigma_{\text {el }} \leq \sigma_{\text {el min }}$ or $\sigma_{\text {el }} \geq \sigma_{\text {el max' }}$ the material will still yield. However, for cohesionless materials $\sigma_{\text {el } \min }=0$, so that the criterion for non-yielding is simply $\sigma_{\mathrm{e} 1}<\sigma_{\mathrm{el}} \max$. In this case, an additional shear stress would need to be applied to the material to produce yield.

The theory that the electrical clamping stress acts as an additional external stress on the material may be analysed using the method of superimposition of Mohr's circles described above. Experimentally, it was found that for a constant mechanical load, application of the electric field required higher shear forces to achieve yield in the material. Therefore, if the predictions of the electrical clamping force models are correct, the new Mohr's circle, resulting from the superimposition of the electrical Mohr's circle onto the mechanical Mohr's circle should lie below the yield locus. This superimposition has been carried out for an electric field strength of $800 \mathrm{kV} \mathrm{m}^{-1}$ applied to the glass ballotini initially in a yielding stress state under application of a normal mechanical stress of 
$2.0 \mathrm{kN} \mathrm{m}^{-2}$. From equation (4.16), the value of $\sigma_{\mathrm{el} \max }$ for $\mathrm{w}_{1}$ equal to 2.0 $\mathrm{kN} \mathrm{m}^{-2}$ is $2.3 \mathrm{kN} \mathrm{m}^{-2}$. A value of $7.4 \mathrm{kN} \mathrm{m}^{-2}$ is predicted from the Elastic Cap model for an electric field of $800 \mathrm{kV} \mathrm{m}^{-1}$. The predicted value of $\sigma_{\mathrm{el}}$ is much larger than $\sigma_{\text {el }}$ max, so the new Mohr's circle would cross the zero field yield locus which would mean that the material yields on application of the electric field. This is clearly inconsistent with the experimental observations.

It is evident from the above analyses that the electrical clamping models fail to predict the shear stresses which have been measured experimentally. In the case of internal yield, simplifying assumptions have been made concerning the number and direction of interparticle contacts, and the distribution of electric current through the bed, in order to estimate the bulk electrical stress on the shear plane from the single contact force models. In reality, the electric current, and thus the electrical clamping forces, may not be distributed according to the simple directional relationship assumed in the model. The current will follow the paths of least resistance which involve the fewest contacts and the largest contact areas. Therefore, the current paths will not only depend on the arrangement of the packing but also on the distribution of mechanical load in the bed. Abdel-Ghani et al. [66] and Travers et al. [67] have found that mechanical stresses in a packed bed are transmitted in discrete chains of particles making many contacts redundant. In this case, it would be necessary to use assembly calculations to solve for the complex interaction of the mechanical and electrical forces in the bed, as discussed above. Considering this possibility, the simplifying assumptions in the internal shear stress analysis may have contributed to the error in the predictions. However, this is not the case for the wall shear analysis where the direction and distribution of the contacts and the distribution of electric current are well-defined. Thus, this analysis is considered to be accurate. In fact, the similarity between the wall and internal yield results suggests that the assumptions in the internal yield analysis are not unreasonable.

Two main reasons could be forwarded for the discrepancy between the predicted shear stresses and the experimental results. First, the single contact force models have themselves not been verified by direct measurement and therefore may be invalid. In particular, the results have shown that the electrical clamping force is greatly sensitive to the 
contact cap size. Therefore, an error in the modelling of the contact cap size could potentially have a large effect on the predictions of the electrical clamping models. From Table 4.1, it can be seen that by including the contribution of the electrical clamping contact force in determining the Hertzian contact cap radius, the electrical clamping stress is reduced by one order of magnitude. The electrical clamping force is modelled as loading the contact point according to the Hertz theory, i.e. taking the same functional relationship as of mechanical loading. Since the electrical clamping force is in fact acting on the particle surfaces across the gap outside the contact area, the deformation caused by the electrical clamping force may take a different functional form from that of the mechanical loading. A larger contact cap size than that predicted from the Hertz theory is needed to bring the theoretical predictions into agreement with the experimental data. The dependance of the contact area on these forces needs therefore to be investigated in order to resolve the validity of the current electrical clamping force models.

Second, the basic assumption in the above analyses has been that the electric field is acting to produce an additional compressive stress on the particles, and that the contact frictional behaviour of the material remains unchanged. However, this method of representing the bulk manifestation of the electrical clamping effect at interparticle contacts may be incorrect. From the argument above, it is speculated that any deformation which is induced by attractive surface forces causes much weaker clamping effect than that predicted by the present models which are based on Hertzian deformation. Therefore, the contribution of the electrical force could be mainly by modifying the adhesive nature of the contact and its resistance to shear, without causing a significant compressive stress. The two alternatives of modelling the effect of the electrical clamping forces as an externally applied stress or as internal cohesion are considered below in the analysis of arching. However, as will be seen in Section 4.4, the main problem in the discrepancy between the experimental and theoretical results, which is manifested on both macroscopic and microscopic scales, lies in the shortcomings of the theoretical models of the electrical clamping force. The issue of interpreting the phenomenon by considering that it modifies the stress state or the yield criterion, albeit very important in the formulation of the macroscopic relationships, cannot explain the discrepancy, and therefore forms a secondary issue in the analysis of the behaviour of 
particulate solids under an electric field. For this reason, later studies were concentrated on experimentally characterizing the electrical clamping force at a single contact, as will be shown in Chapter 5.

\subsection{ARCHING IN HOPPERS}

The arching experiments presented in Chapter 3 were carried out in a wedge-shaped hopper because the electric field distribution and mechanical stress distribution are well defined in this geometry. The wedge-shaped hopper with electrodes as the side walls is shown schematically in Fig. 4.4. With an electric potential applied across the electrodes, the magnitude of the electric field, $\mathrm{E}_{\mathrm{a}}$, is described by equation (4.17).

$$
E_{a}=\frac{V}{2 \kappa r}
$$

where $V$ is the applied voltage across the two electrodes and $\kappa$ is the hopper half angle. In this configuration, the field lines are circular arches which intersect normally with the walls and which have centres at the vertex of the hopper. Therefore, due to the directionality of the electrical clamping forces, the net clamping force should be along the field lines, with zero force perpendicular to the field lines.

There are several models for the mechanical stresses in a wedge-shaped hopper in the literature [46]. The simplest models are based on a force balance over a differential slice in the hopper. Walker [48] and Walters [47] developed their models from a force balance over a horizontal slice. The theory of Enstad [50] uses a force balance over an arched shape element. Due to the arched shape of the electric field lines, Enstad's method of arched differential elements is particularly suitable for superimposing the electrical and mechanical stresses in the hopper and therefore will be used here.

In Enstad's modelling of stresses, he considers two cases. The first case describes the stresses developed in a material in a fully plastic state of stress, which is the case for cohesionless non-consolidating materials. Therefore, this case is suitable for modelling the interaction of the mechanical and electrical stresses in the hopper if the electrical stresses are assumed to act as an externally applied stress without affecting the 
cohesion in the material. Enstad's second case describes cohesive materials which are not in a fully plastic state of stress, i.e. the material has not been allowed to flow sufficiently to reach a steady state. Therefore, this second case is suitable for modelling the interaction of the mechanical and electrical stresses in the hopper if the electrical clamping forces are assumed to produce a cohesion in the material without affecting the net stress state. Both alternative approaches are considered below for modelling the arch holding voltages in the wedge-shaped hopper, starting with the externally applied electrical stress approach where the material is cohesionless and in a fully plastic state of stress.

\section{External Stress Arch Model}

As shown in Fig. 4.4 for passive stresses, Enstad considers that the material in the hopper is made up of arched layers, with no shear stresses transmitted between layers. The major and minor principal stresses are constant in magnitude along a layer. The layers are sections of circles with centres on the centre-line of the hopper. The major principal stress is tangent to the arch and the minor principal stress is normal to the arch for the passive state and vice versa for the active state. Therefore, for the passive stress state the direction of the major principal stress determines the arch line and for the active stress state, the arch line follows the direction of the minor principal stress. The directions of the stresses, and thus the shape of the arch, are specified by the angle $\beta$ which is defined as the angle between the major principal stress plane and the wall. The value of $\beta$ is obtained from a Mohr circle analysis as given in Appendix 4.2 with the result that

$$
\beta_{a}=\frac{1}{2}\left(\phi+\nu_{a}\right)
$$

and

$$
\beta_{p}=\frac{1}{2}\left(\phi+v_{p}\right)
$$

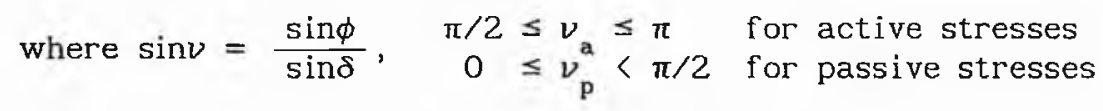

In equations (4.18-4.20), the subscripts $a$ and $p$ refer to the active and passive stress states respectively. In equation (4.20), $\phi$ is the angle of 
wall friction and $\delta$ is the angle of internal friction.

For a fully smooth wall, ie. $\phi=0, \beta_{p}$ will equal zero so that the major principal stress will be normal to the hopper wall. For the passive case, the direction of the major principal stress defines the arch shape in Enstad's mechanical stress analysis and therefore, the stress arches and the electric field arches are coincident for the fully smooth walled case. For a fully smooth wall, $\beta_{\mathrm{a}}$ will equal $\pi / 2$ so that the major principal stress will be tangent to the wall and the minor principal stress will be normal to the wall. For the active case, the direction of the minor principal stress defines the arch shape in Enstad's analysis, so that the stress arches and the electric field arches are also coincident for the active case.

For the fully rough walled case, i.e. $\phi=\delta, v=\pi / 2$ and the stress state may be defined as either active or passive. As given by equation (4.20), Enstad has arbitrarily chosen the active state to be assigned to the fully rough walled case. The designation of the fully rough walled case as either active or passive does have an effect on the analysis, because the shape of the stress arch is determined by the major principal stress direction in the passive case and by the minor principal stress in the active case. Since the angle between the major and minor principal stresses is $\pi / 2$, the shape of the arch will be significantly different between the active and passive cases for the fully rough walled hopper.

The mechanical forces acting on one of Enstad's layers consist of the weight of the layer, $\Delta W$, a net downward force from the material on either side of the layer, $\Delta F_{2}$, and the lift from the walls, $\Delta F_{1}$. The incorporation of the electrical force into the force balance over the layer is complicated by the fact that the electric field lines and the mechanical stress arches are only coincident for fully smooth walls, in which case the net electrical clamping force is acting tangentially to the arch with zero contribution normal to the arch. Therefore, for fully smooth walls the sole contribution of the electrical clamping forces is to produce an additional lifting force at the walls.

For rough walls, there is some misalignment of the electric field lines and the mechanical stress arches. The misalignment will increase as the roughness of the wall increases. The misalignment will also be larger for 
the passive case than for the active case for a particular value of $\phi$. For the passive case, the angle between the wall normal and the arch line at the wall is equal to $\beta_{p}$. For the active case where the minor principal stress direction defines the arch shape, the angle between the wall normal and the arch line at the wall is equal to $\pi / 2-\beta_{a}$. It can be seen that the misalignment given by $\beta_{p}$ in the passive case is larger than the misalignment given in the active case by $\pi / 2-\beta_{\mathrm{a}}$ in the following manipulations of equations (4.18-4.20). From equation (4.20),

$$
v_{\mathrm{a}}=\pi-v_{\mathrm{p}}
$$

Substituting equation (4.21) into equation (4.18) gives

$$
\beta_{\mathrm{a}}=\frac{1}{2}\left(\phi+\pi-v_{\mathrm{p}}\right)
$$

Rearranging equation (4.19) gives

$$
v_{\mathrm{p}}=2 \beta_{\mathrm{p}}-\phi
$$

Finally, substituting equation (4.23) into equation (4.22) gives

$$
\pi / 2-\beta_{\mathrm{a}}=\beta_{\mathrm{p}}-\phi
$$

From equation (4.24), the angle between the arch line and the normal to the wall for the active case is equal to $\beta_{p}-\phi$ which is smaller than the angle between the arch line and the wall normal for the passive case by an amount $\phi$. Therefore the misalignment between the electric field lines and the mechanical stress arches for rough walls is larger for the passive case than for the active case.

In the arching model developed here, the misalignment of the electric field lines and the mechanical stress arches is considered to cause a negligible contribution to the net vertical force in the bulk as compared to the contribution which the electric field makes by providing a lift at the walls. This assumption allows a relatively simple force balance to be carried out where only the electrical clamping force at the walls is added to the mechanical force balance developed by Enstad. The force balance over the arched layer yields 


$$
\Delta W+\Delta F_{2}=\Delta F_{1}+\Delta F_{e}
$$

where $\Delta \mathrm{F}_{\mathrm{e}}$ is the vertical component of the electrical lifting force per unit depth. From Fig. 4.4,

$$
\Delta \mathrm{F}_{\mathrm{e}}=2 \sigma_{\mathrm{e}} \Delta \mathrm{r} \sin \kappa
$$

where $\sigma_{e}$ is the electrical stress at the walls.

The theoretical expression for $\sigma_{e}$ has already been given in equation (4.7). Substituting equations (4.7) and (4.17) into equation (4.26) gives

$$
\Delta \mathrm{F}_{\mathrm{e}}=\frac{2 \alpha}{\mathrm{s}^{2}} \Delta \mathrm{r} \sin \kappa\left(\frac{\mathrm{V}}{2 \kappa r}\right)^{\gamma}
$$

The solution of equation (4.25) for cohesionless materials is given in Appendix 4.2 using equation (4.27) for $\Delta F_{e}$ and the equations for $\Delta W, \Delta F_{1}$, and $\Delta \mathrm{F}_{2}$ as given by Enstad [50]. The result is an expression for the mean principal stress, $\sigma$, which is a function of the voltage difference between the walls, $V$, and the height of the material along the centre-line of the hopper given by $r$.

$\sigma(r)=\frac{\Gamma Y r}{X-1}-\frac{C}{\gamma+X}\left(\frac{V}{r}\right)^{\gamma}+\left\{\sigma(H)-\frac{\Gamma Y H}{X-1}+\frac{C}{\gamma+X}\left(\frac{V}{H}\right)^{\gamma}\right\}\left(\frac{r}{H}\right)^{X}$

where $\mathrm{X}, \mathrm{Y}$, and $\mathrm{C}$ are defined differently for the active and passive stress states. The expressions for these parameters are given in Appendix 4.2. The value of $\sigma(H)$ represents the stress surcharge exerted on the top layer of material in the hopper, eg. as would be produced by material in a bin above the hopper. The value of $\Gamma$ is given by

$$
\Gamma=g \cdot \rho_{\mathrm{d}}
$$

where $g$ is gravitational acceleration and $\rho_{d}$ is the bulk density of the material.

The criterion for formation of a stable, free surface, as is observed to form on application of a sufficiently high electric field to a bed of 
granular material, is that the mechanical stress normal to that surface must equal zero. For the passive case, this stress is the minor principal stress, $\sigma_{2}$, and for the active case, this stress is the major principal stress, $\sigma_{1}$. Therefore, using Mohr circle geometry

$$
\text { or } \quad \begin{aligned}
& \sigma_{2}=\sigma(1-\sin \delta)=0 \\
& \sigma_{1}=\sigma(1+\sin \delta)=0
\end{aligned}
$$

for the passive and active cases respectively. The solution to equations (4.30) and (4.31) requires that either $\sigma$ equals zero or that $\sin \delta$ equals 1 or -1 . The latter equality requires that the internal angle of friction, $\delta$, is equal to $\pi / 2$ or $3 \pi / 2$ which are both unrealistic values. Therefore, the only solution to equations (4.30) and (4.31) is for $\sigma$ to equal zero. Setting $\sigma$ equal to zero in equation (4.28), with $\sigma(H)$ set to zero since there was no surcharge on the top surface of the material in the hopper, results in the following expression:

$$
V=\left\{\frac{\Gamma Y}{C} \frac{(\gamma+X)}{(X-1)}\left(\frac{H^{1-X} r^{X}-r}{H^{-(\gamma+X)} r^{X}-r^{-\gamma}}\right)\right\}^{1 / \gamma}
$$

Equation (4.32) describes the voltage required to produce a stable, stress free surface as a function of the height in the hopper, $r$. In the arching experiments presented in Chapter 3, the stable, free surface was formed at the orifice of the hopper. The height of the hopper at the orifice, $r_{0}$, is related to the orifice width, $b_{o}$, such that

$$
r_{0}=\frac{b_{0}}{2 \sin \kappa}
$$

In Fig. 4.5 to 4.8 , the theoretical predictions for arch holding voltage obtained from equation (4.32) are compared to the experimental results which were presented in Chapter 3. The theoretical predictions are given for the Elastic Cap model of the electrical clamping force as defined by the equations of Moslehi and Self [16] given in Appendix 4.1. The previous analysis of wall and internal shear stresses showed that contact deformation is dominated by the electrical clamping force. Therefore, the use of the Fixed Cap model is not considered here. Figures 4.5 and 4.6 compare active predictions to experimental data for smooth and rough walls respectively, and Fig. 4.7 and 4.8 compare passive predictions to experimental data for smooth and rough walls respectively. 
The arching model presented in equation (4.32) cannot be used to predict holding voltages for the vertical walls case, i.e. $\kappa=0^{\circ}$, because the height of the hopper orifice will equal infinity in this representation. Therefore, the holding voltages for a $1^{\circ}$ hopper half angle have been shown in Figs. 4.5 to 4.8 for approximate comparison with the experimental data of the vertical walls.

As given in Chapter 3, the internal angle of friction of the glass ballotini was $20^{\circ}$ and the wall angles of friction for the smooth and rough walls were $16^{\circ}$ and $20^{\circ}$, respectively. The value of $\Gamma$ was determined to be $15000 \mathrm{~kg} \mathrm{~m}^{-2} \mathrm{~s}^{-2}$ by measuring the bulk density of the glass ballotini. The top surface of the bed of material in the hopper was kept contant at 0.545 m. A particle spacing at the wall of two times the particle radius was assumed in the model calculations, as suggested previously by Tüzün et al. [62] for closely packed spheres in contact with a wall surface.

The theoretical predictions using the Limited Field model of Dietz [19] (see Appendix 4.1) for the electrical clamping force have not been shown because the voltages predicted are two to three orders of magnitude greater than the experimental values. Therefore, the Limited Field model is vastly underpredicting the contribution of the electrical clamping forces towards the formation of a stable arch.

In Figs. 4.5 to 4.8 , the experimental results and the theoretical predictions show that the holding voltage increases with gap width. As the gap width is increased, the mechanical stresses on the arch increase. Therefore, higher voltages are required to provide the additional electrical lifting force needed to bring the normal stress on the arch to zero.

The experimental results show that the holding voltage increases with wall smoothness. This would seem to follow logically, since the mechanical lifting force from the walls decreases as the walls become smoother, and therefore a higher electrical lifting force is required to support an arch. In order to assess the effect of wall roughness on the model predictions, the theoretical holding voltages for the smooth and rough walls are compared in Figs. 4.9 and 4.10. Figures 4.9 and 4.10 show the Elastic Cap predictions for the active and passive stress states respectively. For the 
active stress state in Fig. 4.9, the theoretical holding voltages agree with the trend shown by the experimental data since the holding voltages for the rough walled hopper are less than the smooth walled hopper. For the near vertical wall theoretical values, the difference between the rough and smooth walled hoppers is slight, but for the $10^{\circ}$ and $20^{\circ}$ hoppers, it is significant. For the passive stress state in Fig. 4.10, the trend in the theoretical holding voltages with respect to wall roughness does not agree with that shown experimentally. There is only a very small difference in the holding voltages predicted for the smooth and rough walls, and in most cases, the voltages for the rough walled hopper are slightly larger than the smooth walled hopper.

To determine whether this apparent anomaly in the passive arching model is a result of including the electrical clamping stress in Enstad's mechanical stress model, the stress profiles without any contribution from the electrical clamping forces were calculated [50]. The mean principal stress as a function of height in the hopper, $r$, is plotted for the active and passive cases in Figs. 4.11 and 4.12, respectively. In the active case, Fig. 4.11 shows that the stress in the hopper decreases with increasing angle of wall friction, as expected. In the passive case, Fig. 4.12 shows that the stress in the hopper decreases as the angle of wall friction is increased from $0^{\circ}$ to $15^{\circ}$, but then starts increasing again as the angle of wall friction is increased above $15^{\circ}$. It is not obvious why the stress in the hopper should realistically follow such a trend. Certainly, the experimental data for arching with an electric field dispute this behaviour.

Without actually measuring the stresses, it is difficult to ascertain which stress state is most nearly approached in the hopper, and therefore it is difficult to assess the quality of the theoretical holding voltage predictions in Figs. 4.5 to 4.8 . In the arching experiments, the holding voltages were measured for a hopper filled with material which had not yet been allowed to flow. In this case, it is generally assumed that the stress state is active because the wall friction has not been fully mobilised, however even slight vibrations can cause a switch in stress state for a cohesionless material [42]. The magnitude of the holding voltages predicted by the active arching model in Figs. 4.5 and 4.6 shows better agreement with the magnitude of the experimental values than the predictions of the passive arching model in Figs. 4.7 and 4.8 , which 
supports the existence of an active stress state.

The most significant discrepancy between the experimental results and the theoretical predictions is the sensitivity to hopper half angle. The model predictions are much more sensitive to hopper half angle than the experimental results. The experimental data show that there is only a small increase in holding voltage as the hopper half angle increases. None of the theoretical cases in Figs. 4.5 - 4.8 show this trend with hopper half angle. For each case the behaviour which disagrees most with experiment is the decrease in holding voltage when the hopper half angle is increased from $1^{\circ}$ to $10^{\circ}$. The decrease in holding voltage for the active case is relatively small and the values of holding voltage still agree well with the experimental data. However, the decrease in holding voltage from $1^{\circ}$ to $10^{\circ}$ hopper half angle is very large for the passive stress state, disagreeing completely with the sensitivity to hopper half angle shown by the experimental data. When the hopper half angle is then increased from $10^{\circ}$ to $20^{\circ}$, the active predictions in Figs. 4.5 and 4.6 are in relatively good agreement with experiment. The holding voltages increase with increased angle. The sensitivity to angle is too high, but the actual magnitudes of the holding voltages are close to the measured values. The passive predictions for holding voltage in Figs. 4.7 and 4.8 for $10^{\circ}$ and $20^{\circ}$ show little sensitivity to hopper angle but the magnitude of the predictions is far too small compared to experimental data. From the above evaluation, the results of the active arching model agree better with the experimental data than the passive arching model in magnitude and in their sensitivity to wall roughness and hopper half angle.

Differences between the arching predictions and experimental results may be due to simplifying assumptions in the theory. The sensitivity to hopper half angle may have been decreased in the experimental data by the influence of the front and back walls of the hopper on the stresses in the material. These walls may have been contributing to the support of the vertical stresses in the material. Enstad's stress model is two dimensional and ignores the stresses in the direction normal to the front and back walls. In the arching experiments, the gap between the front and back walls was $12 \mathrm{~mm}$ which is in the range of 10 to 20 particle diameters. This gap size is considered to be suitable to maintain predominantly two-dimensional stress and flow distributions in the material. However, there will always be some influence of the front and back walls on the flow 
and stresses, and this may have affected the sensitivity to hopper half angle of the experimental results as compared to the theory. Another simplification in the theory is the fact that misalignment of the electric field lines and the mechanical arching lines was ignored so that the electric field had no contribution to the forces in the bulk of the bed in the hopper. Considering these simplifications, the active theoretical results in Figs. 4.5 and 4.6 show good agreement with theory.

However, it is important to consider that, although the arching model developed above gave a mathematical solution, in reality the condition of zero normal stress on the arch without failure of the surface for a cohesionless material is physically impossible. The arguments for this statement were given in Chapter 3. Also discussed in Chapter 3 was the existence of dielectrophoretic forces at the orifice of the hopper. The discontinuity of the electrode surface at the hopper orifice in the form of metal tips produces a high electric field gradient in this region. This field gradient produces dielectrophoretic forces which act on the particles in the direction of higher field gradients. At the orifice, the mechanical stress on the material is very small since most of the weight is supported by the mechanical and electrical lift from the walls. Therefore, the action of dielectrophoretic forces on the last few layers of particles immediately above the free surface, pulling them towards the electrode tips, may be sufficient to support the free surface in absence of cohesion. However, the dielectrophoretic forces for chains of touching spheres is very difficult to calculate because of the large number of multipolar interactions which must be considered [68]. Therefore, the significance of this effect is difficult to evaluate.

The arching model developed above is based on the assumption that the electrical clamping forces produce an externally applied stress on the material without affecting the interparticle cohesion between the particles. As discussed previously, the formation of a stable, stress-free surface may alternatively be explained by the existence of interparticle cohesion. The holding voltages predicted by the arching model based on the externally applied stress approach are not in good agreement with the experimental values. The discrepancies may be due to simplifications in the model, as discussed above, or the error may be of a more fundamental nature if the electrical clamping forces are producing a cohesion in the material and not simply an externally applied stress. This possibility was 
tested by developing an alternative arching model based on an electrically produced cohesion within the material using Enstad's approach for modelling the stresses in a cohesive material which is not in a fully plastic state of stress [50].

\section{Electrical Cohesion Arch Model}

As for the External Stress model, the Electrical Cohesion model is developed from a force balance over the arched differential elements of Enstad [50]. The forces acting on the arched element with cohesion are different from those in the External Stress model because the yield locus is different. As shown in Fig. 4.13(a), the internal yield locus for the External Stress model is a straight line through the origin. The Mohr's circle which describes the stresses in the material touches the internal yield locus at one point of tangency. In this case, the major and minor principal stresses are related according to the following:

$$
\sigma_{1}=\frac{(1+\sin \delta)}{(1-\sin \delta)} \sigma_{2}
$$

For the case of a cohesive material, the yield locus no longer passes through the origin but has a negative intercept on the $\sigma$-axis as shown in Fig. 4.13(b). As for the case of no cohesion, the material is considered to be in a state of incipient failure, i.e. an infinitely small increase in orifice size, or in this case, an infinitely small decrease in voltage will cause the material to flow. Therefore, the yield locus touches the Mohr's stress circle at one point of tangency. The yield locus is assumed to be a straight line, i.e. the internal angle of friction is constant. In this case, the major and minor principal stresses are related according to the following equation which is developed in Appendix 4.2:

$$
\sigma_{1}=f_{c}+\frac{(1+\sin \delta)}{(1-\sin \delta)} \sigma_{2}
$$

where $f_{c}$ is the unconfined yield stress of the material. The unconfined yield stress is the major principal stress given by the Mohr's circle which touches the yield locus at one point and which passes through the origin. The minor principal stress of this Mohr's circle is zero. Therefore, the Mohr's circle describes the stress state of a stable, free surface. From Fig. 4.13(b), the unconfined yield stress can be expressed as a function of the cohesion, $c$, as 


$$
f_{c}=\frac{2 c \cos \delta}{(1-\sin \delta)}
$$

For the arching experiments performed for this work, the cohesion of the material was produced by the application of an electric field. In Chapter 3 , it was shown that the internal yield loci of the glass ballotini could be described according to the relationship

$$
\tau=\mu \sigma_{\mathrm{m}}+\mathrm{c}_{\mathrm{e}}
$$

where $\mu=\tan \delta$ and $c_{e}$ is the electrically induced cohesion. In Chapter 3, the experimental data for internal yield were fit to equation (4.37) for three electric field strengths. It was found that the value of $\mu$ was essentially unchanged by the application of the electric field with a value of 0.36 , which gives an angle of internal friction of $20^{\circ}$ for the material. The electrical cohesion increased with increasing electric field as shown in Table 3.1. Using equation (4.36), the values of $f_{c}$ have been calculated from the values of $c_{e}$ given in Table 3.1 and are shown plotted as a function of electric field strength in Fig. 4.14. From Fig. 4.14, it appears that the unconfined yield stress can be described as a linear function of electric field strength such that

$$
\mathrm{f}_{\mathrm{c}}=\mathrm{mE}_{\mathrm{a}}+\mathrm{b}
$$

where $m$ and $b$ are the slope and $y$-intercept respectively of the best fit straight line to the data. For the straight line in Fig. 4.14, $m$ equals $8.66 \times 10^{-4} \mathrm{kN}(\mathrm{kV} \mathrm{m})^{-1}$ and $\mathrm{b}=5.21 \times 10^{-2} \mathrm{kN} \mathrm{m}^{-2}$. Since the glass spheres used in these experiments exhibited no cohesive behaviour without an applied electric field, the small non-zero intercept on the $f_{c}$-axis in Fig. 4.14 is an experimental artifact. Therefore, the value of $b$ was taken to be zero in subsequent calculations. Substituting equation (4.17) for the electric field into equation (4.38) gives an equation for the unconfined yield stress as a function of applied voltage such that

$$
f_{c}=m\left(\frac{v}{2 \kappa r}\right)+b
$$

Finally, substituting equation (4.39) into equation (4.35) results in the following expression for the major and minor principal stresses as a function of applied voltage which can then be used to solve the force 
balance on the arched element:

$$
\sigma_{1}=\left\{\mathrm{m}\left(\frac{\mathrm{V}}{2 \kappa \mathrm{r}}\right)+\mathrm{b}\right\}+\frac{(1+\sin \delta)}{(1-\sin \delta)} \sigma_{2}
$$

The forces acting on the arched element consist of the weight of the layer, $\Delta W$, the net downward force from the material on either side of the layer, $\Delta \mathrm{F}_{2}$, and the lift from the walls, $\Delta \mathrm{F}_{1}$. The force balance on the element is

$$
\Delta \mathrm{W}+\Delta \mathrm{F}_{2}=\Delta \mathrm{F}_{1}
$$

The solution of equation (4.41) for the passive stress state is given in Appendix 4.2 with the final result that

$\sigma_{2}(r)=-\frac{\Gamma Y r}{1-X}-\frac{Z_{r}^{-1}}{1+X}-\frac{Z_{0}}{X}+\left(\frac{r}{H}\right)^{X}\left(\sigma_{2}(H)+\frac{\Gamma Y}{I-X} H+\frac{Z}{1+X} H^{-1}+\frac{Z_{0}}{X}\right)$

The definitions of the functions $X, Y, Z$ and $Z_{0}$ are given in Appendix 4.2, but the definition of $\mathrm{Z}$ will also be given here because the effect of the applied voltage on the stress is included in this function.

For the passive case,

$$
z=\frac{m V}{2 \kappa}\left(\frac{1}{2}+\frac{1}{2} \frac{\sin (2 \omega+\kappa)}{\sin \kappa}\right)
$$

In equation (4.43), $\omega$ is the angle formed between the abutment of the arch surface at the wall and the wall normal. In the analysis of the stresses in a cohesive material, Enstad uses the angle $\omega$ rather than $\beta$ to describe the abutment of the arch against the wall. In Enstad's analysis of the stresses developed in a non-cohesive material, the angle $\beta$ is obtained from a geometrical analysis of the intersection of the wall yield locus with the Mohr's stress circle. In the case of a cohesive material which is not flowing, the material will not be in a fully plastic state of stress and the angle of friction at the wall will not necessarily be fully developed. Therefore, Enstad assumes that the formation of arches is random and defines $\omega$ such that the arched elements get the maximum lift from the walls without the material sliding along the wall. According to this criterion, 
only the passive stress state is relevant in this analysis since in the passive state, where the major principal stress follows the arch line, the arch will get a larger lift from the walls than in the active state, where the minor principal stress follows the arch line. Therefore, the solution for the active stress state is not relevant.

Therefore, based on a passive analysis, Enstad argues that for the layer across the outlet, $\omega$ is limited by the wall friction angle $\phi$. A larger value of $\omega$ would cause the material to slide along the wall until a layer with a smaller $\omega$ were formed. However from Appendix 4.2, equation (A4.70) for the lifting force $\Delta F_{1}$ shows that $\Delta F_{1}$ has its maximum when $\sin (2 \omega+\kappa)$ has its maximum which occurs when $2 \omega+\kappa=\pi / 2$ and thus limits the value of $\omega$. Therefore, $\omega$ is defined such that

$$
\omega=\begin{array}{ll}
\phi & \text { if } \phi \leq \omega_{\mathrm{m}} \\
\omega_{\mathrm{m}} & \text { if } \phi>\omega_{\mathrm{m}}
\end{array}
$$

where $\omega_{\mathrm{m}}=\frac{\pi}{4}-\frac{\kappa}{2}$

For the hopper half angles used in the arching experiments, $0^{\circ}, 10^{\circ}$, and $20^{\circ}$, the values of $\omega_{\mathrm{m}}$ are $45^{\circ}, 40^{\circ}$, and $35^{\circ}$. Therefore, for the arching experiments, the angles of wall friction for both the smooth and rough walls, $16^{\circ}$ and $20^{\circ}$ respectively, are smaller than $\omega_{\mathrm{m}}$. Therefore according to equation (4.44), the value of $\omega$ is equal to the angle of wall friction, $\phi$.

The cohesion model for arching is obtained by setting $\sigma_{2}$ in equation (4.42) equal to zero to meet the criterion for the stress-free surface of a stable arch, and solving for the critical value of $Z$ to produce arching, $Z_{c}$, such that

$$
Z_{c}=\frac{\frac{\Gamma Y}{1-X}\left\{\left(\frac{r}{H}\right)^{X} H-r\right\}+\frac{Z_{o}}{X}\left\{\left(\frac{r}{H}\right)^{X}-1\right\}+\left(\frac{r}{H}\right)^{X} \sigma_{2}(H)}{\left\{-\frac{r^{-1}}{1+X}-\left(\frac{r}{H}\right)^{X} \frac{H^{-1}}{1+X}\right\}}
$$

Finally, the model for the arch holding voltage is expressed by rearranging equation (4.43) and setting $Z=Z_{c}$. Therefore, 


$$
V=\frac{2 \kappa Z_{c}}{m}\left(\frac{1}{2}+\frac{1}{2} \frac{\sin (2 \omega+\kappa)}{\sin \kappa}\right)^{-1}
$$

The results of equation (4.46) for arch holding voltage are compared to the experimental data in Figs. 4.15 and 4.16 for rough and smooth walls, respectively. As in the case of the External Stress Arch model, a hopper half angle of $1^{\circ}$ is used in the Electrical Cohesion Arch model to compare with the experimental results for the $0^{\circ}$ hopper half angle. The theory correctly predicts that increased holding voltages are required as the orifice width is increased and the mechanical stresses on the arch increase. Similarly to the External Stress model, the holding voltages predicted by the Electrical Cohesion model show a much stronger dependency on hopper half angle than the experimental data show. However, the model predictions do follow the correct trend, showing holding voltages increasing with increasing hopper half angle. As shown in Fig. 4.17, the model predictions also correctly predict higher holding voltages for the smooth walled hopper than for the rough walled hopper. However, the magnitude of the holding voltages predicted by the Cohesion model are not in good agreement with the experimental data. Figs. 4.15 and 4.16 show that the holding voltages are underpredicted by the model, with the error being most significant for the smooth walled hopper.

The External Stress model suffered from several simplifications as discussed above. The Electrical Cohesion model is also based on a major simplifying assumption. The relationship between the unconfined yield stress and the electric field strength was based on internal yield loci measured for one direction of electric field for which maximum resistance to shear deformation was expected. As discussed previously, it is expected that the application of an electric field to a granular material produces an anisotropic distribution of electrical clamping forces within the material. Therefore, the yield loci will depend on the direction of the electric field. Since it would be extremely complicated to solve for the stress distribution in a material with anisotropic cohesion, the cohesion model was developed by assuming isotropic cohesion within the material. Since the values of $f_{c}$ used in the model are maxima, it would be expected that holding voltages would be underpredicted as is the case in Figs. 4.15 and 4.16.

Because the stress state in the hopper is indeterminate, ie. active or 
passive, it is impossible to distinguish between the merits of the two models based on which one fits the experimental data better for the correct stress state. Overall, the External Stress model in the active state fits best, but considering only the passive state, the Electrical Cohesion model fits better. In fact, both the External Stress and Cohesion models give very similar agreement with experiment. The first model was based on the theoretical models of the electrical clamping force which were found to give huge discrepancies between theory and experiment in the shear cell analysis in Section 4.3. The second model was based on the actual experimental shear cell data. Therefore, it appears that the stress analyses developed for the hopper in this Section are not sensitive to the electrical clamping forces. Thus, it is not possible to conclude from the arching analyses whether the external stress or cohesion approach describes the effect of the electrical clamping forces correctly. The two main issues remain unsolved concerning the modelling of macroscopic behaviour in the presence of electrical clamping forces. First, are the single contact force models valid, and second, should the electrical clamping forces be treated as external applied forces or as cohesive forces.

\subsection{STEADY-STATE FLOW}

In order to model the steady-state flowrate through the EVS as a function of the continuous electric field, it is first necessary to consider the flow through the electrode assembly in the absence of a field. Since the upstream electrode has been found to have no detectable effect on the flow, the problem is essentially one of modelling the flow through the oversized mesh of the downstream electrode [69]. By considering the individual mesh openings in the downstream electrode as non-interacting orifices, the flowrate through the entire grid can be obtained by adding the flow through each opening. Here, the widely accepted correlation of Beverloo et al. [70] is used to estimate the flowrate through an individual mesh opening. This correlation gives the flowrate through a single circular orifice in a flat-bottomed hopper as

$$
W=c_{f} p_{f} g^{1 / 2}\left(D_{o}-k d_{p}\right)^{5 / 2}
$$

where $W$ is the mass flowrate, $\rho_{f}$ the flowing density, $g$ the gravitational acceleration, $D_{a}$ the diameter of the orifice, $d_{p}$ the diameter of the 
particles, $c_{f}$ the coefficient of flow, and $k$ a constant which depends on particle shape. Nedderman et al. [71] have found that the value of $c_{f}$ is constant and equal to 0.58 for a wide range of materials, and that $\mathrm{k}$ ranges in value from 1.5 for smooth particles to 2.5 for angular particles. In equation (4.47), the term $\left(D_{0}-k d_{p}\right)$ can be regarded as an effective orifice opening, where the diameter of the orifice is reduced by an amount equal to $\mathrm{kd}_{\mathrm{p}}$ due to the presence of an 'empty annulus' around the perimeter of the orifice where no particles can flow.

The Beverloo correlation can be modified to describe a general orifice shape by rewriting equation (4.47) in terms of an effective area $A^{*}$, and a hydraulic diameter $\mathrm{D}_{\mathrm{h}}{ }^{*}$ such that

$$
W=\frac{4}{\pi} c_{f} \rho_{f} A^{*}\left(g D_{h}^{*}\right)^{1 / 2}
$$

For a square mesh opening of side length $d_{0}$, as relevant to the cylindrical column electrode design,

$$
A^{*}=\left(d_{0}-k d_{p}\right)^{2}
$$

and

$$
\mathrm{D}_{\mathrm{h}}^{*}=\mathrm{d}_{\mathrm{o}}-\mathrm{kd}
$$

Similar relationships can be developed for the parallel wire slot configuration in the rectangular duct shown in Fig. 1.1.

By combining equations (4.48) to (4.50), an equation is obtained for the flowrate through one square mesh opening, $\mathrm{w}_{\mathrm{m}}$, such that

$$
W_{m}=\frac{4}{\pi} c_{f} \rho_{\hat{f}} g^{1 / 2}\left(d_{o}-k d_{p}\right)^{5 / 2}
$$

The total equivalent number of mesh openings, $n$, making up a circular grid of diameter $\mathrm{D}_{\mathrm{c}}$ can be determined by taking into account the wire diameter $\mathrm{d}_{\mathrm{w}}$ : 


$$
\mathrm{n}=\frac{\pi}{4}\left(\frac{\mathrm{D}_{\mathrm{c}}}{\mathrm{d}_{\mathrm{o}}+\mathrm{d}_{\mathrm{w}}}\right)^{2}
$$

Therefore, the total flow through the column, $W_{c^{\prime}}$ is obtained by multiplying the flow through each mesh opening in equation (4.51) by the number of mesh openings in equation (4.52):

$$
W_{c}=c_{f} \rho_{f} g^{1 / 2}\left(\frac{D_{c}}{d_{o}+d_{w}}\right)^{2}\left(d_{o}-k d_{p}\right)^{5 / 2}
$$

Equation (4.53) can be rearranged into the following linear form to facilitate fitting of the parameters $c_{f}$ and $k$ to the experimental data:

$$
\left[W_{c}\left(\frac{d_{o}+d_{w}}{D_{c}}\right)^{2}\right]^{2 / 5}=\left[c_{f} \rho_{f} g^{1 / 2}\right]^{2 / 5} d_{o}-\left[c_{f} \rho_{f} g^{1 / 2}\right]^{2 / 5} k d_{p}
$$

The quantity on the left hand side of equation (4.54) is equivalent to $\left(w_{m} \frac{\pi}{4}\right)^{2 / 5}$, where $w_{m}$ is the flow through one mesh opening. Therefore, equation (4.54) essentially describes the relationship between the flow through each individual mesh opening and the size of the mesh opening, $d_{0}$.

Experimental data for flow through different sized meshes with no field applied are plotted in Fig. 4.18 according to equation (4.54). Two column sizes of 64 and $115 \mathrm{~mm}$ were used with two sand sizes of $600-1200 \mu \mathrm{m}$ and 1200-2400 $\mu \mathrm{m}$. The linear relationship of equation (4.54) is shown to be valid. The values of $c_{f}$ and $k$ for each line were calculated from a linear best fit analysis and are given in Table 4.2, where the bulk flowing density of the material is $1.6 \times 10^{3} \mathrm{~kg} \mathrm{~m}^{-3}$. Except for one case, i.e. the $115 \mathrm{~mm}$ column with 0.6-1.2 $\mathrm{mm}$ sand, the values of the parameters agree reasonably well with the values given by Nedderman et al. [71]. However, contrary to the assumption of non-interacting orifices, the results in Fig. 4.18 show that the flow through a single mesh opening depends on the column diameter. The difference in the results for the two columns probably arises from the incomplete mesh openings around the perimeter of the columns, which are formed by fixing a square mesh across a cylindrical column. This feature is not accounted for in equation (4.52), where the 
cross-sectional area of the column is assumed to consist only of complete square openings. However, because of the 'empty annulus' effect, the total area of the incomplete openings provides less available flow area than square openings of equal total area. Indeed some of the incomplete openings are too small for any flow at all. Therefore, the number of effective square openings contributing to the flow is actually less than the theoretical number $\mathrm{n}$ given by equation (4.52). The group on the left of equation (4.54) therefore underestimates the flow per mesh opening, and this effect will increase with decreasing column diameter (i.e. increasing periphery/area). The results in Fig. 4.18 support this analysis since the lines for the $64 \mathrm{~mm}$ column fall below and deviate from those of the $115 \mathrm{~mm}$ column as the mesh opening size is increased. Nevertheless, the results in Fig. 4.18 are sufficient to show that the dependence of the flowrate on the mesh size can be adequately described by the modified Beverloo correlation.

To extend the mesh flow model to include the effect of the electric field on flow, an analogy has been made with the effect of interstitial gas pressure gradients on discharge of fine granular materials from hoppers and bins. Nedderman et al. [33] have shown that the Beverloo correlation can be modified to account for the effect of air drag on discharge from a conical hopper as

$$
W \propto g^{1 / 2}\left(1+\left.\frac{1}{g \rho_{f}} \frac{d P}{d r}\right|_{\Gamma=r_{0}}\right)^{1 / 2}
$$

where $P$ is the interstitial pressure, $r$ is the distance from the virtual apex of the cone, and $r_{0}$ is the value of $r$ at the orifice. According to equation (4.55), flow under gravity is reduced by the gradient of interstitial gas pressure at the orifice, which provides a net force on the particles opposite to the gravitational force. An attempt has been made to apply a similar functional relationship for the effect of the electric field, without implying that a similar mechanism is operating. In fact, it is not clear at present whether the retardation of flow by the electric field is due to an upward force counteracting gravity or due to an increased shear viscosity of the flowing particles. Experimental observations of flow behaviour have so far not produced a clear picture. Therefore, here only an attempt is made to explore a dependence of flow on electric field strength of the form: 


$$
W \propto g^{1 / 2}\left(1-\lambda E_{a}^{\xi}\right)^{1 / 2}
$$

where equation (4.56) follows the functional form of equation (4.55), and the term describing the effect of the electric field follows the functional form of the electrical clamping force given by equation (4.4). The constants $\lambda$ and $\xi$ are determined from experiment data. From equations (4.53) and (4.56), the flowrate through the column with an applied field follows as

$$
W=W_{0}\left(1-\lambda E_{a}^{\xi}\right)^{1 / 2}
$$

where $W$ is the flowrate with an applied electric field and $W_{0}$ is the flowrate without an applied field, which can be measured or predicted from equation (4.53) if the values of $c_{f}$ and $k$ have been previously determined for the EVS geometry in use. For convenience in fitting parameters $\lambda$ and $\xi$ to the experimental data, equation (4.57) has been rearranged as

where $Y=1-\left(\frac{W}{W_{0}}\right)^{2}$

$$
\mathrm{Y}=\lambda \mathrm{E}_{\mathrm{a}}^{\xi}
$$

Equation (4.58) has been fitted to a large set of experimental data for W, $W_{0}$ and $E_{a}$, collected from both the cylindrical and rectangular columns, using a non-linear least squares routine to obtain values of $\lambda$ and $\xi$. Figures 4.19 and 4.20 compare the experimental values of $Y$ for the data in Figs. 3.11 and 3.12 to the best fit values from equation (4.58), showing that very good fits are obtained for individual data sets. However, when all the data sets are compared, the values of $\lambda$ and $\xi$ cover wide ranges, with no apparent trends related to the experimental conditions. For example, for the $115 \mathrm{~mm}$ column with $0.6-1.2 \mathrm{~mm}$ sand and a lower electrode mesh opening size of $10 \mathrm{~mm}$, the best fit values of $\xi$ and $\lambda$ from 9 sets of data cover ranges of $1.4-3.4$ and $1.3 \times 10^{-4}-2.1 \times 10^{-10} \mathrm{~m}^{\xi} \mathrm{kV}^{-\xi}$, respectively. In collecting these data, the only aspects of the test conditions which were deliberately varied were the design of the upper electrode and the spacing between the upper and lower electrodes.

Two aspects of the test conditions which were not kept constant during the particular tests mentioned above were the relative humidity, which ranged 
from 40-60\%, and the sand size distribution, which would have changed due to attrition in the pneumatic conveying line used to refill the cylindrical columns. As noted by Johnson and Melcher [18], experimental observations confirmed that relative humidity affects the flow response of some materials, e.g. sand, $\mathrm{NaCl}$ salt, and glass ballotini, where the main source of surface conduction is through adsorbed moisture. In particular, the holding voltages of these materials increase as the relative humidity decreases. The particle size distribution of the material will have a physical effect on the flow as predicted by equation (4.53), and in addition, it will affect the electrical response of the flow behaviour, due to the sensitivity of the electrical clamping forces to the size of the particles as well as the size of the contact area between particles as given by the electrical clamping force models.

In order to determine whether the wide ranges of $\lambda$ and $\xi$ noted above were due to variations in relative humidity and particle size distribution, a series of tests was conducted where these two parameters were kept within a narrow range. The flowrate response of finely-sized 600-710 $\mu \mathrm{m}$ glass ballotini was measured in the rectangular column under relative humidity and temperature of $50 \pm 5 \%$ and $20 \pm 2^{\circ} \mathrm{C}$, respectively. The pneumatic conveying system was not used with the rectangular column, so attrition was negligible. In these tests, the electrode configuration shown in Fig. 1.1 was used with various spacings for the upper and lower electrode wires. The range of values of $\xi$ and $\lambda$ for the 11 tests are $0.88-1.5$ and $4.0 x$ $10^{-3}-1.7 \times 10^{-4} \mathrm{~m}^{\xi} \mathrm{kV}^{-\xi}$, respectively. While the ranges of $\xi$ and $\lambda$ are much narrower than for the tests with sand, there is still no apparent reason for the spread of the values.

The form of equation (4.58) is such that, with the values of $\lambda$ and $\xi$ unconstrained, it is always possible to achieve a good fit to the data. Fig. 4.21 shows the results of an error sensitivity analysis for varying $\lambda$ and $\xi$ for the fit of equation (4.58) to the data shown in Figs. 3.11 and 4.19 for the $115 \mathrm{~mm}$ column, demonstrating that the sum of the squares of the errors does not reach a sharply defined mimimum where the best fit is described by a unique set of values for $\lambda$ and $\xi$. In combination, a wide range of values of $\lambda$ and $\xi$ can be used to give an error close to the overall minimum error of 0.049 for $\xi$ equal to 2.2 . Therefore, the flow model given by equation (4.57) cannot be tested rigorously with the available data. 
As an alternative to equation (4.57), the relationship between flowrate and voltage may be described on a purely empirical basis by considering that the trend in the data shown in Figs. 3.11 and 3.12 appears to follow a parabolic or elliptical shape. However, empirically-fitted relationships of these forms produce fits which are overall no better or worse than equation (4.58), so that the flow model given by equation (4.58) and parabolic and elliptical correlations are equally suitable for calibrating the EVS for practical operation.

The approach outlined above for modelling the steady-state flow in the EVS is simplistic, since it uses the average value of the electric field without considering the effect of the highly non-uniform fields produced in the complex electrode configurations used here. The sensitivity of the EVS response to different electrode configurations was examined qualitatively in Chapter 3 where it was shown in Figs. 3.14 and 3.15 that different flowrate responses are produced by changing the geometry of the upstream electrode or the separation between the electrodes and thus changing the electric field distribution between the electrodes. In these cases, where a highly non-uniform field is present, it is clear from the results shown in Figs. 3.14 and 3.15 that it is not sufficient to use the average field to model the flow response. In future, it is necessary to proceed with a more rigorous analysis, with precisely defined electrical and mechanical stress distributions incorporated into the macroscopic constitutive equations of motion. However before this analysis can be undertaken, the questions arising out of the much simpler analyses of wall and internal failure, and static arching must be resolved through verification of the electrical clamping force models.

\subsection{CONCLUSIONS}

The analyses of the interaction of the electrical clamping forces with the prevailing mechanical forces in the yield stress and arching work clearly indicate the difficulty associated with the transformation of the microscopic contact forces to that of a macroscopic stress representation of the bulk. Two important issues have emerged from these analyses, one relating to the validity of the current electrical clamping force models, and the other relating to the nature of the macroscopic representation of 
the electrical clamping phenomenon, i.e. treating it as an external force.

First, the electrical clamping force models have not so far been validated by single contact force measurements, and as shown in this work, there is a difficulty in the interpretation of data, obtained from macroscopic measurement of bulk parameters, in inferring values for the electrical clamping force. Furthermore, from single contact mechanics, it is not clear why the electrical clamping force should produce a Hertzian deformation, as assumed in all the present electrical clamping models. Here, the interparticle force is generated over the surfaces in the interparticle gap outside the contact area, where a highly non-uniform electric field prevails. It is not immediately obvious that this field would give rise to a Hertzian pattern of deformation. Because the clamping force is very sensitive to the contact area, other patterns of deformation could lead to a substantially different magnitude of the electrical clamping force. For example, if the deformation is induced by a force akin to adhesion, the force of adhesion is completely independent of the elastic moduli of the materials, and the contact area has a finite value depending on the solid surface energy so that Hertzian deformation is not applicable [72].

Second, the treatment of the electrical clamping force as an external compressive force implies that the increased bulk shear resistance is brought about by an equivalent external load such that the frictional properties of the bulk remain unaltered by the electric field. There is however no direct experimental evidence showing this unequivocally, and because of the difficulty associated with the single contact electrical clamping force models, it cannot be verified here either. Furthermore, the electrically induced arch formation in cohesionless particles in hoppers indicates that the treatment of the electrical force as an equivalent external stress field cannot explain the formation of stress free surfaces, as observed below a stable arch. However, this phenomenon can be described by the existence of an unconfined yield stress, thus suggesting that the electric field modifies the cohesive/adhesive nature of the contact without necessarily increasing the normal load. If the electrical clamping forces do act as cohesive forces, it is then necessary to consider the influence of the electrical force in modifying the contact frictional behaviour of the particles by increasing the intrinsic shear resistance of an adhesive contact, taking into account a realistic mode of contact surface 
deformation.

In order to develop and verify any new models for the single contact electrical clamping force and accompanying deformation, the results presented here have shown that bulk scale measurements are not sufficient for inferring single contact behaviour. Thus, the measurement of the electrical clamping force at a single contact was undertaken as the final aspect of this research. The results of this work are presented in Chapter 5 . 


\section{CHAPTER 5}

\section{CHARACTERIZATION OF THE SINGLE CONTACT ELECTRICAL CLAMPING FORCE}

\subsection{INTRODUCTION}

The analysis of the macroscopic bulk behaviour in Chapter 4 showed that attempts to model the observed behaviour in shear cells using the single contact electrical clamping force models were not successful. Since the electrical clamping force models have so far not been experimentally verified, it is possible to conclude that the source of error in the theoretical bulk predictions is the single contact models or the method of the assembly calculations. However, based on the analysis of wall failure where the assembly calculations are considered to be accurate, it is believed that the discrepancy in the bulk predictions originates from incorrect models of the electrical clamping force. The only conclusive way to prove that this is the case is to directly measure the electrical clamping force at a single contact.

This measurement requires a very sensitive force measuring device, because although the net stress generated by the individual electrical clamping forces in an assembly of many particles is significant, the force at one contact is small. A suitable device was available from another research programme investigating the surface hardness of materials at nanometre penetration depths under very small compressive loads. The device is called the Nanotest and is manufactured by MicroMaterials Ltd., UK. Originally developed by Pollock et al. [34], the Nanotest is capable of measuring forces as small as $10 \mu \mathrm{N}$ and displacements as small as $10 \mathrm{~nm}$ [73]. In order to use the Nanotest to measure the electrical clamping force, modifications were made to the device as described below. The resulting technique was used to measure the electrical clamping force at detachment for single contacts between glass spheres and a conductive wall [74]. The experimental results are compared to the predictions of the electrical clamping force models below. In view of the deficiencies of these models, an alternative model for the electrical clamping force based on fracture mechanics theory is discussed [31]. Finally, attempts to quantify the contact size produced by the electrical clamping force are presented. 


\subsection{EXPERIMENTAL METHOD}

\section{Description of device}

A schematic diagram of the modified Nanotest device is shown in Fig. 5.1. The main component is an alumina pendulum which is mounted on frictionless pivots. A copper coil is attached to the top of the pendulum. When an electric current is applied to the copper coil, the coil is attracted or repelled from an electromagnet which is positioned next to the coil. The direction of the force depends on the polarity of the current. The movement of the copper coil causes the pendulum to swing in the desired direction to apply a compressive or tensile force on the particle.

For the electrical clamping force measurements, a particle is mounted in place of the diamond indentor used in the surface hardness measurements. The particle is mounted on a stainless steel pin which is secured to the pendulum as shown in Fig. 5.1. The method of mounting is described further below.

The displacement of the particle is measured by means of a parallel plate capacitor. One plate of the capacitor is attached to the pendulum and the other plate is fixed next to the pendulum. When the pendulum moves, the capacitance between the plates changes, and this change is measured with a capacitance bridge. The capacitance bridge unit is located close to the measuring capacitor in order to minimise stray capacitance effects.

The electrical clamping force measurements described below were carried out for contacts between lead glass spheres, supplied by Jencons Scientific Ltd. UK, and a polished stainless steel plate. The plate was mechanically polished first with silicon carbide paper and then with diamond particles sprayed on cloth down to a maximum asperity height of $1 \mu \mathrm{m}$. When the stainless steel plate was viewed with a Scanning Electron Microscope, it could be seen that the surface was essentially smooth down to a resolution of about $1 \mu \mathrm{m}$. Occasional scratches could be found on the surface, as shown in Fig. 5.2, but these were very few. The stainless steel plate was connected to the EHT supply so that it formed the high voltage electrode. The stainless steel plate was glued to a ceramic rod which was then mounted on a moveable stage. The ceramic rod was used to insulate the stainless 
steel plate from the rest of the device. The steel plate was brought into contact with the particle by controlling the position of the stage through the computer software. The pin on which the particle was mounted was earthed by connecting a very thin copper wire between the pin and an earthed point on the device.

The equilibrium position of the pendulum with no load current applied to the copper coil is adjusted with balance weights which are movable along the horizontal and vertical axes. The limit stop is used to determine the maximum outward movement of the lower part of the pendulum when a load current is applied to the coil. This is used to ensure contact in the presence of a small mechanical load before the electric field is applied to the contact.

\section{Calibration of the applied mechanical force and displacement}

The data for the mechanical force applied to the particle and the displacement of the particle are obtained from the binary data acquired by the computer via the Analogue/Digital Converter or from chart recorder traces of the coil voltage and the capacitance bridge outputs.

The mechanical force applied to the particle when it is pushed into contact with the steel plate or pulled away from the plate is determined from the voltage applied to the copper coil, which generates a current and hence a magnetic force, $F_{m}$, to move the pendulum. The calibration between the force on the particle and the voltage applied to the copper coil to move the pendulum is carried out first by measuring the voltage required to swing the pendulum back into its equilibrium position against the limit stop when a dead weight is hung onto the end of the balance weight arm as shown in Fig. 5.3. This measurement is made for a range of weights from 1 to $2.5 \mathrm{~g}$. The value of this calibration factor, WV, is found by fitting a straight line to the data for the mass of the dead weight, $m_{w}$, versus the voltage applied to the coil, $V_{c}$. Therefore, $W V$ is equal to $d\left(m_{w}\right) / d\left(V_{c}\right)$. The value of $W V$ is dependent on the position of the permanent balance weights mounted on the pendulum, and on the position of the limit stop and weight of the mounted test sphere. In this work these parameters varied little from day to day testing and the calibration factor WV fell in the range of $0.0304-0.0341 \mathrm{~g} \mathrm{mV}^{-1}$. A typical calibration curve is shown in Fig. 5.4 . 
With the pendulum in equilibrium, the force applied by the electromagnet is related to the force produced on the pendulum from the dead weight by

$$
F_{m} \cdot y_{1}=M_{w} \cdot x
$$

where $W_{w}$ is the weight of the dead weight, and the distances $x$ and $y_{1}$ and the force $F_{m}$ are defined schematically in Fig. 5.3.

In normal testing, the calibration weights are removed from the pendulum and the test sphere is brought into contact with the plate. A normal force, $f_{n}$, is applied to the contact by applying a current to the pendulum coil which forces the lower part of the pendulum to swing out and push the sphere against the plate. The applied force, $f_{n}$, is determined from the electromagnetic force on the coil from the relationship

$$
f_{n} \cdot y_{2}=F_{m} \cdot y_{1}
$$

where the distance $y_{2}$ is defined in Fig. 5.3. By combining equations (5.1) and (5.2), the force on the sphere can be related directly to the force of the calibration weight hung on the pendulum by

$$
f_{n}=\frac{M_{w} \cdot x}{y_{2}}
$$

The load calibration factor, LCF, between the force on the particle and the coil voltage is given by $\mathrm{d}\left(\mathrm{f}_{n}\right) / \mathrm{d}\left(\mathrm{V}_{e}\right)$. The value of LCF is found from the calibration factor WV and equation (5.3) such that

$$
\mathrm{LCF}=\frac{\mathrm{WV} \cdot \mathrm{x} \cdot \mathrm{g}}{\mathrm{y}_{2}}
$$

where $g$ is gravitational acceleration. In this work, the value of $x$ and $y_{2}$ were 24.5 and $29.0 \mathrm{~mm}$ respectively. Therefore, the value of LCF ranged from $0.252-0.283 \mathrm{mN} \mathrm{mV}^{-1}$.

In the experiments to measure the single contact electrical clamping force, the force data were monitored and stored using a chart recorder to record the voltage output from the coil. The output of the chart recorder was calibrated to the coil output, $V_{c}$, which was then related to the force on the particle using the value of LCF. 
Displacement is determined by calibrating the capacitance bridge output with the displacement of a linearly variable differential transformer (LVDT). First, the steel plate is moved into contact with the sphere. Then the LVDT probe is fixed to the same frame as the fixed capacitor plate and placed in contact with the steel plate so that it will indicate movement of the steel plate in the horizontal direction. The steel plate is then moved in very small steps so that the gap between the capacitor plates changes. After each step, the voltage output from the capacitance bridge and the voltage output from the LVDT are noted. The calibration factor, CR, between the voltage output from the LVDT and the capacitance bridge rectified output is found by fitting a straight line to the data. A typical calibration curve is shown in Fig. 5.5. The value of CR depends on the gain setting of the device. In this work, the value of $\mathrm{CR}$ ranged from $0.0427-0.0661 \mathrm{mV}$ (LVDT) $\mathrm{mV}^{-1}$ (Rec O/P). The depth calibration factor, $\mathrm{DCF}$, for the actual displacement as a function of capacitance bridge output is obtained by multiplying $C R$ by the voltage output response factor for the LVDT, VR. The value of VR was specified by the manufacturer Schlumberger as $1281 \mathrm{~nm} \mathrm{mV}{ }^{-1}$. Therefore the value of DCF ranged from 54.7 - 84.7 $\mathrm{nm} \mathrm{mV}^{-1}(\operatorname{Rec} \mathrm{O} / \mathrm{P})$.

\section{$\underline{\text { Specimen mounting }}$}

For attachment to the pendulum, the test particle is mounted on a stainless steel pin with $1 \mathrm{~mm}$ diameter. The particles used in this work were glass spheres of diameter $1.9 \pm 0.02 \mathrm{~mm}$ and $3.7 \pm 0.04 \mathrm{~mm}$. The glass spheres were individually chosen for a high degree of smoothness and sphericity under a microscope at a magnification of 50 times. The glass spheres were attached to the pin with a drop of Superglue. This resulted in some of the Superglue spreading over the pin and over the sphere near the contact. Electrodag (silver paint) was then carefully painted over the Superglue to ensure a conductive circuit between the pin and sphere. The final appearance of the mounted specimen is shown schematically in Fig. 5.6. The extent of Electrodag coverage varied slightly between specimens. For each mounted sphere, the distance left uncovered by Electrodag was measured under a microscope. This distance is defined by $\mathrm{L}$ in Fig. 5.6. When the sphere is in contact with the stainless steel plate, $L$ is the linear distance between the electrodes, i.e. the Electrodag and the plate. Before being attached to the pendulum for a test, the glass bead and pin were 
immersed in a $1 \%$ (by volume) surfactant solution for a few minutes. The pin and glass bead were then removed and allowed to dry by clamping the pin in an upright position. This procedure is similar to the shear cell and arching tests and was adopted to ensure similar surface resistivities.

Fig. 5.7 shows photographs of the surface of a coated glass sphere as viewed under a Scanning Electron Microscope. Compared to the stainless steel plate shown in Fig. 5.2, the surface of the glass sphere is rough with an asperity height in the order of $1 \mu \mathrm{m}$. When the surfaces of coated and uncoated spheres were viewed under the SEM, no difference in surface topography was evident.

\section{Method for measuring electrical clamping force}

The pin and the glass bead are secured to the pendulum in the position shown in Fig. 5.1. The equilibrium position of the pendulum is set by adjusting the positions of the balance weights and the limit stop. The calibration factors for force and displacement are then determined as described above.

The setup for measuring the electrical clamping force as a function of applied electric field is shown schematically in Fig. 5.8. The applied voltage is monitored by a voltmeter via a high voltage probe connected in parallel with the sphere. The current flowing over the sphere is measured with an electrometer connected in series with the sphere.

The first step of the experiment was to bring the stainless steel plate into contact with the glass sphere. Before starting the movement of the plate towards the sphere, a small force is applied on the pendulum by applying a voltage to the copper coil to pull it back against the limit stop into a stable position. With the pendulum fixed in this position, the gap between the capacitance plates and thus the output from the capacitance bridge is held constant. The stage holding the steel plate is then made to move at a very slow speed towards the glass bead. When contact between the plate and the bead is made, the gap between the capacitance plates starts to close and the capacitance bridge output changes. The software driving the device is set to detect this change and stop the movement of the stage. In this way, the particle and the plate can be brought into very light but stable contact. The compressive force acting on the contact is negligible. 
This was found by measuring the detachment force, as explained below, in the absence of an electric field, where the force was found to be below the resolution of the device, $10 \mu \mathrm{N}$. If the pendulum was not first pulled against the limit stop, but was instead allowed to swing freely in its equilibrium position when bringing the particle into contact with the plate, the capacitance bridge output would be too unstable for a very small change in capacitance to be detected.

With the sphere in contact with the steel plate, a voltage is applied between the plate and the pin holding the sphere. From this point on, the steps in the experimental method can best be followed by referring to Fig. 5.9, which shows typical chart recorder traces for the electric current flowing over the sphere and for the force applied to the particle. The chart recorder trace for the electric current is the output from the electrometer. The chart recorder trace for the force applied to the particle is actually the voltage applied to the copper coil on the pendulum. This was calibrated to the force applied to the particle according to the method described above.

Before a voltage is applied to the glass sphere, the chart recorder output from the electrometer shows that there is zero current flowing over the sphere, and the chart recorder output from the copper coil shows the force offset applied to pull the pendulum back against the limit stop. When the voltage is applied, there is an initial overshoot in the current due to the overshoot behaviour of the EHT unit when increasing the voltage. The duration of the overshoot is very short and the current quickly reaches a steady value for the steady applied voltage. The electrical clamping force at the contact between the glass bead and the steel plate now holds the pendulum rigidly in place so the small force offset applied by the electromagnet to maintain stability can now be reduced to zero without affecting the position of the pendulum or the contact. The polarity of the voltage applied to the copper coil is now reversed in order to produce a repulsive force between the copper coil and the electromagnet and cause the pendulum and the particle to pull away from the steel plate. The magnitude of the voltage applied to the copper coil is increased at a steady rate until the contact breaks. In all the tests, the tensile force was increased at a rate of $1.8 \mathrm{mN} \mathrm{min}^{-1}$, which was the minimum rate possible with the device. The point at which the contact breaks can be seen in Fig. 5.9 as the point when the current drops suddenly to zero. By 
observing the contact itself, it can be seen that at this point the contact breaks catastrophically and the pendulum swings suddenly away from the steel plate and oscillates slightly before reaching a rest position. The oscillations of the pendulum cause oscillations in the current as the capacitance of the circuit through the electrometer is momentarily unstable.

The value of the tensile force on the particle at the point of detachment is determined from the chart recorder traces from the value on the force trace corresponding to the point when the current dropped to zero. This tensile force is equal to the electrical clamping force on the particle at the point of detachment.

In the single contact detachment experiments, the current was monitored primarily as a method of determining the detachment point. However, the current measurements could also be used to measure the electrical resistance of the contact. From the electrical setup shown in Fig. 5.8, the current measured by the electrometer is related to the applied voltage across the sphere and the electrical resistance of the sphere by

$$
I=\frac{V}{R_{s}+R_{e l}}
$$

where $I$ is the current measured by the electrometer, $V$ is the applied voltage, $R_{s}$ is the resistance of the sphere and $R_{e l}$ is the internal resistance of the electrometer. By rearranging equation (5.5), the resistance of the sphere can be calculated from the measured current from

$$
R_{s}=\frac{V}{I}-R_{e l}
$$

For these experiments, $R_{e l}$ was always at least three orders of magnitude less than the value of $\frac{V}{I}$ so it could be ignored in equation (5.6).

The electrical clamping force at detachment and sphere resistance were measured for two sizes of glass spheres as mentioned above, $1.9 \mathrm{~mm}$ and 3.7 $\mathrm{mm}$ average diameters. The measurements were made for a range of applied voltages. The lowest voltage which could be applied was $0.4 \mathrm{kV}$ because at lower voltages the response of the EHT was unstable. The electric field strength for gross sparkover between the stainless steel plate and the Electrodag covering on the glass sphere was measured to be $1.9 \times 10^{3} \mathrm{kV} \mathrm{m}^{-1}$ 
for a $3.7 \mathrm{~mm}$ diameter sphere in contact with the stainless steel plate. At approximately $5 \mathrm{kV}$, a spark between the plate and the Electrodag on the back of the sphere was seen. The distance L from Fig. 5.6 was about $2.7 \mathrm{~mm}$ giving an electric field strength of $1.9 \times 10^{3} \mathrm{kV} \mathrm{m}^{-1}$ for gross sparkover. However, corona was generated at field strengths lower than the gross sparkover voltage for the $3.7 \mathrm{~mm}$ spheres, above about $1.1 \times 10^{3} \mathrm{kV} \mathrm{m}^{-1}$. The presence of corona was identified by rapid oscillations in the current signal after the voltage had been applied. The amplitude of the oscillations increased as the voltage was increased. The corona was probably caused by the sharp metal edges of parts of the Nanotest which were near the sphere. To avoid effects of corona, detachment forces were measured up to about $1.1 \times 10^{3} \mathrm{kV} \mathrm{m}^{-1}$ for the $3.7 \mathrm{~mm}$ spheres. The voltage was applied across the gap between the stainless steel plate and the edge of the Electrodag on the sphere. For the $3.7 \mathrm{~mm}$ spheres, this gap was on average $2.7 \mathrm{~mm}$ which gave a maximum applied voltage of about $3 \mathrm{kV}$. For the $1.9 \mathrm{~mm}$ spheres with an average gap size of $1.2 \mathrm{~mm}$, detachment forces were measured up to about $1.6 \mathrm{kV}$ or $1.3 \times 10^{3} \mathrm{kV} \mathrm{m}^{-1}$. A slightly higher maximum field strength was used with the $1.9 \mathrm{~mm}$ spheres because corona did not occur.

The measurements were made under constant temperature and humidity conditions of $20 \pm 2{ }^{\circ} \mathrm{C}$ and $50 \pm 5 \%$ respectively.

\subsection{RESULTS OF SINGLE CONTACT DETACHMENT EXPERIMENTS}

The detachment force measurements are shown in Fig. 5.10 as a function of the electric field strength. The electric field strength, $E_{a}$, was calculated as

$$
E_{a}=\frac{V}{L}
$$

where $L$ is the distance between the steel plate and the edge of the Electrodag coating on the sphere. The detachment forces were measured for three spheres of $1.9 \mathrm{~mm}$ diameter and two spheres of $3.7 \mathrm{~mm}$ diameter. At each applied field, the detachment force was measured twice. From Fig. 5.10, there is very little difference in the two detachment force measurements for a particular sphere at one field strength. There is a larger variation in the detachment forces measured for different spheres of 
the same diameter. However, these differences are not significant considering variations in curvature, roughness, surfactant coating, and relative humidity which might have affected the electrical clamping forces. The results of the detachment force measurements in Fig. 5.10 show that the electrical clamping forces increase with particle diameter, and at first glance appear to increase approximately linearly with the applied electric field strength.

The results of the electrical resistance measurements for the same tests as described above are shown in Fig. 5.11(a) and 5.11(b). Figure 5.11(a) gives the results for two $1.9 \mathrm{~mm}$ spheres and one of the $3.7 \mathrm{~mm}$ spheres. Figure 5.11(b) gives results for the other 1.9 and $3.7 \mathrm{~mm}$ spheres. The data have been spread over two graphs because of the large range of the magnitudes of the resistances. As for the force measurements, the resistance was measured twice at each electric field strength for each sphere. Where only one symbol is present in Fig. 5.11, this implies that the data points overlap. There is almost no variation at all between these duplicate resistance measurements. However, there is a large variation between the resistances measured between different spheres at the same applied field strength. These differences are most likely due to differences in the surface coverage of the surfactant on the glass spheres or asperity contacts. These single contact experiments were meant primarily to provide measurements of the electrical clamping force for a single contact that could be compared to the results of the bulk experiments. No attempt was made to provide spheres with a smoother surface for this reason. The surfaces of the glass spheres were coated in the same way as the glass spheres in the bulk experiments. The glass spheres were not cleaned in any way prior to being coated with the surfactant, so any contaminants on the surface of the spheres could have caused patchy coating of the surfactant. Although the spheres were carefully handled, it is also possible that the surface became contaminated by dust particles after coating. As only one sphere was tested per day, variations in relative humidity would also certainly have caused variations in surface resistivity of the sphere surfaces.

Figure 5.11 shows that the sphere resistances all decrease with increasing field strength and appear to be approaching an asymptotic value. This pattern of response is similar to the dependence of the resistance over a sphere on the contact size given by the contact resistance theory of McLean 
[15] in equation (A4.4) (see Appendix 4.1). McLean's resistance theory is shown below:

$$
R_{\theta}=\frac{\rho_{s}}{2 \pi} \ln \left(\frac{\tan \frac{\theta}{2}}{\tan \frac{\theta_{0}}{2}}\right)
$$

The relationship given in equation (5.8) is plotted in Fig. 5.12 for the contact size expressed in terms of the contact angle $\theta_{0}$, where $\theta_{0}=\frac{a}{R}$ from the contact radius, $a$, and particle radius, $R$. In Fig. 5.12, the sphere resistances have been calculated for varying angles of $\theta$ over the sphere and for a nominal value of 1 Tera $\Omega \mathrm{m} / \mathrm{m}$ for the surface resistivity, $\rho_{\mathrm{s}}$, of the glass. The absolute values of the sphere resistances are unimportant in Fig. 5.12; the curves are shown only to compare the trend in the shape to the measured data in Fig. 5.11. The comparison shows that the decrease in resistance measured for the spheres may be due to increased contact areas as the spheres deform with increasing electric field. It should be pointed out that the trend of the resistance data does not indicate the onset of corona at the contact since the electric field strengths applied in the tests were below that at which corona occurred. Generally, the existence of corona would bring about electrical breakdown which in turn would cause the resistance to decrease with increasing electric field until, at a limit of the electric field strength, the resistance would drop suddenly to zero. This is obviously not the case in our experiments; the resistance data in Fig. 5.11 clearly show approach to asymptotic values.

The chart recorder trace of electric current in Fig. 5.9 shows the current decreasing catastrophically, not gradually, to zero. However, according to JKR fracture mechanics theory of contact detachment under normal loading [72], it is expected that the contact size would decrease to some extent before fracturing at some finite value where the equilibrium energy state becomes unstable. If this were happening in the detachment experiments, the resistance would be expected to increase and the current to decrease during the process of detachment. On the contrary, the sudden drop in current in Fig. 5.9 indicates that the contact is not changing size as the tensile force is being applied to the contact to break it. It is also observed that the displacement of the particle, as monitored through the Nanotest device, remains almost constant as the tensile force is increased during a test, and only begins to change very rapidly immediately before 
the sphere breaks away from the stainless steel plate. Therefore, if the change in contact size is very small prior to the point of separation, the corresponding change in the current could be too small to be detected by the electrometer. From equation (5.8), the rate of change of $R_{\theta}$ with $\theta_{0}$ is given by

$$
\frac{\mathrm{dR}_{\theta}}{\mathrm{d} \theta_{0}}=-\frac{\rho}{2} \frac{\mathrm{s}}{\pi} \frac{1}{\sin \theta_{0}}
$$

Therefore, the change in sphere resistance would only be large at small values of $\theta_{\mathrm{o}}$, as shown in Fig. 5.13 where $\rho_{\mathrm{s}}$ is again set arbitrarily to be 1 Tera $\Omega \mathrm{m} / \mathrm{m}$. The JKR fracture mechanics theory will be discussed in greater detail in Section 5.7 where the size of the contacts produced by electrical clamping forces is analysed.

\subsection{ANALYSIS OF DETACHMENT FORCE}

In Figs. 5.14 and 5.15, the measured values of the detachment force are compared with the predictions from the Elastic Cap model and the Limited Field model for the electrical clamping force given by equations (A4.32-A4.34) and (A4.19) respectively, in Appendix 4.1. In the theoretical calculations, the values of $\nu_{r}$ and $E_{m}$ for lead glass are 0.25 and $6.2 \times 10^{10} \mathrm{~N} \mathrm{~m}^{-2}$ [75] and for stainless steel they are 0.28 and $2.1 \mathrm{x}$ $10^{11} \mathrm{~N} \mathrm{~m}^{-2}$ [76], respectively. The value of $3 \times 10^{6} \mathrm{~V} \mathrm{~m}^{-1}$ was used for $\mathrm{E}_{\max }$ in the Limited Field model [19].

In Figs. 5.14 and 5.15, the measured and predicted values of the electrical clamping force have been divided by $R^{2}$, where $R$ is the particle radius, because in the Elastic Cap and Limited Field models, the electrical clamping force is proportional to $\mathrm{R}^{2}$. It is interesting to note that the experimental data also show the same trend, because the results of the two sphere sizes become coincident when Force $/ \mathrm{R}^{2}$ is plotted as a function of electric field. However, it is clear from Figs. 5.14 and 5.15 that the absolute magnitudes of the theoretical predictions do not agree with the experimental data. The predictions of the Elastic Cap model are one to two orders of magnitude too large and the predictions of the Limited Field model are one to two orders of magnitude too small, when compared to the experimental data. The Fixed Cap model is not applicable here because no mechanical load was applied to the contact so the contact size should be 
determined by the electrical clamping force alone. These discrepancies between the measured and theoretical values for the electrical clamping force at a single contact confirm the results of the analysis of the macroscopic bulk behaviour in shear cells in Chapter 4: the theoretical models for the electrical clamping force are invalid.

It is of interest to compare the experimental values of the detachment forces with the polarization force for a dielectric sphere touching a conductive plate. The formulation for this force was given in Chapter 4 and is repeated here for convenience. From Dietz [56 ]

$$
f_{p}=4 \pi \varepsilon_{f} R^{2} E_{a}^{2} \zeta\left(\frac{3 \eta^{2}}{2(3-\eta)^{2}(1-\eta)}\right)
$$

where $\eta=\frac{\varepsilon_{p}-\varepsilon_{f}}{\varepsilon_{p}+\varepsilon_{f}}$

In equation (5.10), $\varepsilon_{p}$ and $\varepsilon_{f}$ are the absolute permittivities of the particles and interstitial fluid respectively. The value of $\zeta$ is dependent on the value of $\eta$ and is found from a numerical solution given by Dietz [56]. For lead glass with a relative permittivity of approximately 7.0 and air with a relative permittivity of $1, \eta$ has a value of 0.75 and $\zeta$ has a value of approximately 1.18. For the $3.7 \mathrm{~mm}$ diameter spheres with the maximum electric field strength of $900 \mathrm{kV} \mathrm{m}^{-1}$ used in the detachment experiments, the polarization force according to equation (5.10) is 0.24 $\mathrm{mN}$. For the $1.9 \mathrm{~mm}$ diameter spheres with the maximum electric field strength of $1700 \mathrm{kV} \mathrm{m}^{-1}$, the polarization force is $0.23 \mathrm{mN}$. For the $3.7 \mathrm{~mm}$ and $1.9 \mathrm{~mm}$ diameter spheres, the measured electrical forces were approximately $6 \mathrm{mN}$ and $2 \mathrm{mN}$, respectively. The dielectrophoretic forces are therefore one to two orders of magnitude smaller than the measured force, so the force on the sphere cannot be attributed to polarization as the dominating mechanism.

In a very recent study, Mizes [77] has actually measured the polarization forces for very small polymer and glass spheres $(22-37 \mu \mathrm{m}$ diameter) near a conducting plane in an electric field. In his work, the particles were insulated from one of the electrodes so current leakage over the particle was prevented. Mizes used Atomic Force Microscopy to measure the attractive forces between a particle and the plane for varying separation distances between the particle-plane surfaces. He also measured the 
detachment forces required to pull the particle out of contact with the surface. In both cases, he found the force followed a quadratic dependence on electric field strength as expected for polarization forces (see equation (5.10)). In order to compare the detachment forces measured in this work to those of Mizes, it is necessary to extrapolate the experimental data in Figs. 5.14 and 5.15 far out of the range of particle sizes used in the experiments. The experimental data for Force $/ R^{2}$ in Figs. 5.14 and 5.15 follow closely a straight line. A best linear fit through the origin to the data results in the relationship

$$
\mathrm{F}_{\mathrm{e}} / \mathrm{R}^{2}=1.65 \mathrm{E}_{\mathrm{a}}
$$

where the units of $\mathrm{F}_{\mathrm{e}} / \mathrm{R}^{2}$ and $\mathrm{E}_{\mathrm{a}}$ are $\mathrm{N} \mathrm{m}^{-2}$ and $\mathrm{kV} \mathrm{m} \mathrm{m}^{-1}$ respectively. For a $37 \mu \mathrm{m}$ diameter sphere in a field of $25 \mathrm{kV} \mathrm{m}^{-1}$, a detachment force of $14 \mathrm{nN}$ is predicted by equation (5.11). This value is far smaller than the force measured by Mizes, which was about $150 \mathrm{nN}$. Mizes was also able to measure the detachment force due to the natural adhesion of the contact without an electric field. This force was approximately $50 \mathrm{nN}$. Clearly, it is not valid to extrapolate the data measured in this research for comparison to the very small forces measured by Mizes. Not only are Mizes particles 100 times smaller, but even more significantly, his measuring device is far more sensitive than the Nanotest. The Atomic Force microscope could measure forces as small as $0.003 \mathrm{nN}$ and is therefore one million times more sensitive than the Nanotest. Although Mizes experiments are not comparable to this work, the use of the Atomic Force microscope for measuring single contact forces is an important accomplishment and warrants future investigation in the field of the electrical clamping forces. It is also interesting to note that Mizes measured significant fluctuations in the detachment forces due to variations in surface roughness. Surface roughness also appears to affect the electrical clamping forces.

It may be noted that the measured values of the electrical forces for the conditions tested here, i.e. for glass spheres in contact with a stainless steel plate, are not extremely large. Similar magnitudes of forces could be generated by van der Waal's forces if the surfaces were extremely smooth. In their JKR model, Johnson et al. [72] developed a model for adhesive forces acting between two non-conforming surfaces. The tensile force $F_{s}$ required to separate the surfaces is given by 


$$
F_{s}=\frac{3}{2} \gamma_{s} \pi R
$$

where $\gamma_{s}$ is the surface energy of the two surfaces in contact and $R$ is the radius of the sphere. Taking a realistic value of $\gamma_{\mathrm{s}}$ as $0.1 \mathrm{~J} \mathrm{~m}^{-2}[31,72]$, the detachment forces for adhesive contacts are $0.4 \mathrm{mN}$ and $0.9 \mathrm{mN}$ for the $1.9 \mathrm{~mm}$ and $3.7 \mathrm{~mm}$ diameter spheres respectively. These values of detachment forces are similar to those measured at the lowest electric field strengths. However, these adhesive detachment forces would only occur for smooth surfaces, which is not the case for the glass spheres used in these experiments. As mentioned earlier, with zero applied electric field in the detachment experiments described above, the detachment force was measured to be zero within the sensitivity of the device, $10 \mu N$. If the particles were smooth, the van der Waal's forces would make them sufficiently cohesive so as to form stable arches even in the absence of an electric field; the arching experiments in Chapter 3 showed that this was not the case.

\subsection{ANALYSIS OF MACROSCOPIC BEHAVIOUR USING SINGLE CONTACT FORCE MEASUREMENTS}

While the electrical clamping force at a single contact is relatively small, and at low electric fields it is comparable with the van der Waal's force, it can nevertheless change the macroscopic bulk behaviour significantly. This is evident from the bulk experiments discussed in Chapter 3 where the flow behaviour of beds of particles was drastically altered. It is now of interest to re-analyse the macroscopic behaviour from the shear cell and arching experiments using the measured values of the electrical force at a single contact. This is clearly only an approximate evaluation as the conditions of the bulk experiments and the single contact experiments were not the same.

First, the single contact electrical forces were measured under tension so that there was no normal compressive loading of the contacts. The particles in the shear and arching experiments were subject to normal compressive loads. The electrical clamping force models predict that the increased deformation at the contacts from this loading should affect the electrical force at the contact. However, the predicted influence of mechanical loading was shown to be small in the analyses of wall and 
internal yield given in Chapter 4 (see Table 4.1). Also, the results of the wall and internal yield experiments did not support this behaviour at the bulk level: the yield loci for different electric field strengths remained parallel over the range of applied normal loads. Therefore, although the effect of normal mechanical loading on the electrical clamping force at a contact remains to be verified, the bulk experimental data presented here suggest that the effect was small in those experiments.

Secondly, the issue arises as to the validity of using a single contact force measured under tension to analyse the bulk compressive forces in the shear cell and arching experiments. However from a force balance approach, the tensile force required to break the contact is equal to the compressive force acting on the contact. As the single contact measurements indicate that the contact is not changing size under application of the tensile force, similarly to the case of brittle crack formation [78], it is a reasonable approximation to use the force balance approach to equate the tensile force to the compressive force acting on the contact.

Finally, the comparison between the bulk data and single contact measurements is not exact because, in the case of the bulk experiments the particles were subject to tangential loads. In particular, in the shear cell experiments shear forces were measured not normal forces. The electrical clamping forces remain to be measured for friction, but in the first instance, it will be assumed that the single contact friction properties are not changed by application of the electric field, i.e. the coefficient of friction remains unchanged as shown by the wall and internal yield loci in Chapter 3. Therefore, the single contact electrical force can be treated as an external applied force additional to any mechanical force on the contact.

The dependency between the electrical clamping force and additional mechanical loading, normal and tangential, remains to be resolved in future work. For the sake of providing an initial comparison between the electrical clamping forces measured at a single contact and those inferred from the bulk experiments, the macroscopic data obtained from the shear cell experiments of Robinson and Jones [32] and from the arching experiments are analysed using the single contact electrical force measurements. The data of Robinson and Jones for wall shear are used rather than the data for internal shear in order to keep the analysis as 
straight forward as possible, since the assumptions about the distribution of the electrical and mechanical stresses and the packing of the particles are more exact for the case of wall shear than for internal shear.

A significant shift of the wall yield loci was measured by Robinson and Jones [32] who interpreted it by considering an electrical stress acting on the bed in addition to the mechanical normal stress. In Table 3.1 the electrical stresses were given for the fit of equation (3.2) to the experimental data for wall yield. Electrical stresses of 72 and $203 \mathrm{~N} \mathrm{~m}^{-2}$ were fit to the data for applied electric fields of 390 and $790 \mathrm{kV} \mathrm{m}^{-1}$, respectively. Since Robinson and Jones found their yield stress measurements to be independent of bed height, the electrical stress must be generated from the electrical clamping forces at the contacts between particles and the wall. The average electrical clamping force at each contact can be determined from the electrical stress by assuming an average spacing between the particles at the wall so that

$$
f_{e}=\sigma_{e} \cdot s^{2}
$$

where $\sigma_{e}$ is the net electrical stress determined from the fit to the data and $\mathrm{S}$ is the average distance between particle centres. Robinson and Jones used glass ballotini with diameters 600 to $710 \mu \mathrm{m}$, so the average particle radius $R$ was $330 \mu \mathrm{m}$. The values of $f_{e}$ from equation (5.13) are shown in Table 5.1 for average particle spacings of $S$ equal to $2 R$ for the closest packed case and $S$ equal to $3 R$ for a reasonable estimate of loose packing. The values of the electrical clamping force measured in the detachment experiments cannot be compared directly with the values inferred from Robinson and Jones' bulk data because of different glass sphere sizes. However, values for the electrical clamping force for particles with $330 \mu \mathrm{m}$ radius can be calculated by extrapolation from the experimental data using equation (5.11). The values of $F_{e}$ calculated using equation (5.11) for $R$ equal to $330 \mu \mathrm{m}$ are shown in Table 5.1. The magnitude of the force values extrapolated from the single contact measurements agree very well with those inferred from Robinson and Jones' yield data for a packed bed. The similarity in magnitude between the bulk measurements and the single contact measurements serves to reinforce the view that the present models of the electrical clamping force are invalid.

The arching of glass spheres in the wedge-shaped hopper has also been 
re-analysed using the results of the single contact experiments. The force balance on the arched differential element given by equation (4.25) is used, where only the expression for the electrical lifting force, $\Delta \mathrm{F}_{\mathrm{e}}$, differs from the analysis given in Chapter 4.

As shown previously in Chapter 4, the vertical component of the electrical lifting force is given by

$$
\Delta \mathrm{F}_{\mathrm{e}}=2 \sigma_{\mathrm{e}} \Delta \mathrm{r} \sin \kappa
$$

where $\sigma_{\mathbf{e}}$ is the electrical stress at the walls. In the theoretical analysis of arching given in Chapter $4, \sigma_{e}$ was given by the theoretical models for the electrical clamping force in equation (4.7). Here, the empirical relationship obtained from the single contact measurements in the form of equation (5.11) is used to describe the electrical stress at the hopper walls. Combining equations (5.11) and (5.13),

$$
\sigma_{e}=\frac{K^{\prime} R^{2} E_{a}}{s^{2}}
$$

where $\mathrm{K}^{\prime}$ is $1.65 \times 10^{-3} \mathrm{~N} \mathrm{~V}^{-1} \mathrm{~m}^{-1}$. Substituting equation (5.15) into equation (5.14),

$$
\Delta \mathrm{F}_{\mathrm{e}}=\frac{2 \mathrm{~K}^{\prime} \mathrm{R}^{2} \Delta \mathrm{r} \sin \kappa \mathrm{E}_{\mathrm{a}}}{\mathrm{S}^{2}}
$$

As shown in Chapter 4, the electric field between the electrodes in the wedge shaped hopper is described by

$$
E_{a}=\frac{V}{2 \kappa r}
$$

Substituting equation (5.17) into equation (5.16) results in the following expression for the electrical lifting force on the arched element in Fig. 4.4:

$$
\Delta F_{e}=\frac{K^{\prime} R^{2} \Delta r \sin \kappa V}{S^{2} \kappa r}
$$

Equation (5.18) is substituted into the force balance over the arched 
element given by

$$
\Delta \mathrm{W}+\Delta \mathrm{F}_{2}=\Delta \mathrm{F}_{1}+\Delta \mathrm{F}_{\mathrm{e}}
$$

The equations for $\Delta W, \Delta F_{2}$ and $\Delta F_{1}$ are unchanged from the analysis given in Chapter 4 for the external stress arching model. Similarly to the theoretical analysis detailed in Appendix 4.2 and discussed in Chapter 4, equation (5.19) is solved for the mean principal stress, $\sigma$, in the hopper for the active and passive stress states. The solution is given by

$\sigma=\frac{\Gamma Y r}{X-1}-\frac{K}{1+X}\left(\frac{V}{r}\right)+\left\{\sigma(H)-\frac{\Gamma Y H}{X-1}+\frac{K}{1+X}\left(\frac{V}{H}\right)\right\}\left(\frac{r}{H}\right)^{X}$

where

$K=\frac{R^{2} K^{\prime}}{2 S^{2} K(1+(z) \sin \delta)} \quad, \quad \begin{aligned} & z=-1 \text { for the passive stress state } \\ & z=+1 \text { for the active stress state }\end{aligned}$

The expressions for $X$ and $Y$ in equation (5.20) are the same as those developed for the External Stress Arch model in Chapter 4 (see Appendix 4.2).

Equation (5.20) is solved for the voltage required to produce a stable arch using the criterion for the normal stress on a free surface given in Chapter 4 by equations (4.30) and (4.31). For the case of no surcharge on the material in the hopper, the voltage required for arching is given by

$$
V=\left\{\frac{\Gamma Y}{K} \frac{(1+X)}{(X-1)}\left(\frac{H^{1-X}{ }^{X}-r}{H^{-(1+X)} r^{X}-r^{-1}}\right)\right\}
$$

The results of the arching model given by equation (5.21) are compared to the experimental data in Fig. 5.16 - 5.19. Figures 5.16 and 5.17 show the holding voltage predictions for the passive stress state for the smooth and rough wall cases respectively. Figures 5.18 and 5.19 show the results for the active stress state for smooth and rough walls respectively. The predicted holding voltages in Fig. 5.18 and 5.19 for the active case are in poor agreement with experiment, being one order of magnitude higher than the experimental data and showing a high sensitivity to hopper half angle. Except for the $1^{\circ}$ hopper half angle, the results in Fig. 5.16 and 5.17 for the passive case are in much better agreement with the experimental data. 
The predicted holding voltages for $10^{\circ}$ and $20^{\circ}$ hopper half angles are still slightly too high, but follow a similar trend with gap size as compared to the experimental data, and show only a small sensitivity to hopper half angle. As was discussed in Chapter 4, the basis of the arching model involves several simplifying assumptions. Further improvement of the arching model requires a more rigorous solution of the electrical and mechanical stress interactions.

\subsection{DISCUSSION ON FRACTURE MECHANICS APPROACH TO ELECTRICAL CLAMPING FORCE}

It should be noted that the models of the electrical clamping force used in the analyses in this Chapter and in Chapter 4 were all developed from a force balance approach. Therefore, at detachment the tensile force applied by the Nanotest machine is assumed to equal the compressive force caused by the electric field. However, during detachment elastic deformation of the contact occurs, which affects the energy state of the system and also modifies the electric field distribution acting in the gap outside the contact. These changes should be accounted for when modelling the electrical clamping force by using a fracture mechanics analysis. Fracture mechanics theory takes into account the energy lost or gained as the sphere deforms, while a force balance analysis does not. Kendall [31] has recently applied the fracture mechanics method of analysis to the electrical clamping force generated at the contact between two cylinders of dielectric material as shown in Fig. 5.20. The geometry is essentially that of an annular crack in a cylinder. When a voltage potential is applied across the two cylinders, an attractive force is generated by the constriction of current through the contact between the bodies, similarly to the spherical geometries considered in this work.

Kendall uses the analysis of the force generated by an electric field across two parallel surfaces given by Stuckes [36] to describe the attractive electrical stress across the surfaces in the crack:

$$
\sigma_{\mathrm{a}}=\left(\frac{\varepsilon_{\mathrm{f}}}{8 \pi}\right) \mathrm{E}_{\mathrm{a}}^{2}
$$

where $\varepsilon_{f}$ is the permittivity of the fluid in the gap, in this case air, and $E_{\mathrm{a}}$ is the average electric field strength across the gap. The tensile 
stresses needed to break contacts between discs were measured and equated to the attractive pressures in equation (5.22) following a force balance approach. Kendall considers this equality to be incorrect because it does not take into account the change in the gap height due to elastic deformation as the tensile stress is applied. He proposes an alternative method of analysis which is reviewed below.

When a tensile load is applied in the absence of an electric field, the gap can be considered as a crack which can propagate through the contact, breaking the adhesive join between the cylinders. When no electric field is applied, this adhesive join would exist to some extent due to surface forces such as van der Waal's forces. Kendall modelled the force required to break this adhesive contact by applying a fracture mechanics approach following the Griffith energy balance concept [79]. By considering the total energy in the system, $U_{t}$, as a function of the contact diameter, $d$, the equilibrium condition for a reversible crack will occur when

$$
\frac{d\left(U_{t}\right)}{d(d)}=0
$$

Equation (5.23) describes the condition for detachment of the two cylinders. The total energy $U_{t}$ in the system is made up of three terms, the surface energy, $U_{s}$, the stored elastic energy in the deformed material, $\mathrm{U}_{\mathrm{e}}$, and the amount of work done by the applied load, $U_{p}$.

The surface energy is

$$
\mathrm{U}_{\mathrm{s}}=-\frac{\pi \mathrm{d}^{2}}{4} \gamma_{\mathrm{s}}
$$

where $\gamma_{s}$ is the interfacial surface energy required to separate the two surfaces in contact and $d$ is the diameter of the contact.

The stored elastic energy is

$$
\mathrm{U}_{\mathrm{e}}=\int \mathrm{F}_{\mathrm{t}} \mathrm{d} \delta_{\mathrm{n}}
$$

where $F_{t}$ is the applied tensile force and $\delta_{n}$ is the deformation of the bodies normal to the contact given by 


$$
\delta_{n}=\frac{F_{t}\left(1-v_{r}^{2}\right)}{E_{m}^{d}}
$$

where $v_{\mathrm{r}}$ is the Poisson's ratio and $\mathrm{E}_{\mathrm{m}}$ is the elastic modulus of the cylinder material. Using equation (5.26) to solve equation (5.25) results in

$$
U_{e}=\frac{F_{t}^{2}\left(1-v_{r}^{2}\right)}{2 E_{m}^{d}}+A
$$

where A is an arbitrary constant.

The work done by the applied load is given by

$$
U_{p}=-F_{t} \delta_{n}+B
$$

where $B$ is an arbitrary constant. Substituting equation (5.26) into equation (5.28) results in

$$
U_{p}=-\frac{F_{t}^{2}\left(1-\nu_{r}^{2}\right)}{E_{m}^{d}}+B
$$

where comparison of equation (5.29) and equation (5.27) shows that $\mathrm{U}_{\mathrm{p}}=-2 \mathrm{U}_{\mathrm{e}}$, which applies for any body under constant applied stress during crack formation [78].

Solving equation (5.23) from the total energy $U_{t}$ given by the summation of equations (5.24), (5.27) and (5.29) results in the following expression for the tensile force necessary to break the contact:

$$
F_{t}=\left(\frac{\pi \gamma_{s} E_{m} d^{3}}{\left(1-v_{r}^{2}\right)}\right)^{1 / 2}
$$

From equation (5.30), the stress required to break the contact is given by

$$
\sigma_{c}=\frac{4 F_{t}}{\pi D^{2}}=\left(\frac{d^{2}}{D^{2}}\right)\left(\frac{16 E_{m} \gamma_{s}}{\pi d\left(1-v_{r}^{2}\right)}\right)^{1 / 2}
$$

where $\mathrm{D}$ is the diameter of the cylinder. Equation (5.31) describes the 
tensile stress required to break the contact of the two cylinders without an applied electric field. With an applied field, the additional pressure which must be compensated for in detachment is given by equation (5.22) in Kendall's analysis [31]. Since the gap height between the cylinders will change as the detachment force $F_{t}$ is applied and the bodies deform, the electric field $E_{a}$ in equation (5.22) must be expressed as a function of the gap height, h. For two cylinders, Kendall uses the Boussinesq tensile stress field to calculate the gap height as a function of the applied tensile load, $F_{t}$ :

$$
h=\frac{4 F_{t}\left(1-\nu_{r}^{2}\right)\left(1-\frac{2}{\pi} \sin ^{-1} \frac{d}{2 r_{c}}\right)}{E_{m}^{d}}
$$

where $r_{c}$ is the radial distance from the centre of the cylinders.

When a voltage potential is applied between the two cylinders, there will be a potential distribution along the surfaces outside the contact due to the increased resistance near the contact. Considering an overall voltage potential of $2 \mathrm{~V}$ across the cylinders, the potential difference across the gap is given by Kendall from Lamb's potential theory (see [31]) as

$$
V_{h}=2 V\left(1-\frac{2}{\pi} \sin ^{-1} \frac{d}{2 r_{c}}\right)
$$

where it is assumed that volume conduction rather than surface conduction is operating.

Using equations (5.32) and (5.33), the electric field strength across the gap outside the contact is given by

$$
E_{a}=\frac{V_{h}}{h}=\frac{V E_{m} d}{2 F_{t}\left(1-v_{r}^{2}\right)}
$$

It is important to note from equation (5.34), that in this particular geometry the electric field is constant in the gap and does not vary with position. For the spherical configurations used in this work, this is not the case; the electric field is highly nonuniform in the gap, increasing significantly in strength towards the edge of the contact.

From equations (5.22) and (5.34), the pressure acting across the surfaces 
outside the gap due to the electric field is given by

$$
\sigma_{e}=\left(\frac{\varepsilon_{f}}{8 \pi}\right)\left(\frac{V E_{m} d}{2 F_{t}\left(1-v_{r}^{2}\right)}\right)^{2}
$$

With application of an electric field, the total stress, $\sigma_{t}$, required to break the contact must now be increased from the value given in equation (5.31) to

$$
\sigma_{t}=\sigma_{c}+\sigma_{e}=\left(\frac{d^{2}}{D^{2}}\right)\left(\frac{16 E_{m} \gamma_{s}}{\pi d\left(1-v_{r}^{2}\right)}\right)^{1 / 2}+\left(\frac{\varepsilon_{f}}{8 \pi}\right)\left(\frac{V E_{m} d}{2 F_{t}\left(1-v_{r}^{2}\right)}\right)^{2}
$$

When the adhesive surface energy $\gamma_{s}$ is small, the detachment stress is dominated by effect of the electric field. Therefore, assuming $\gamma_{s}=0$ in equation (5.36) gives the following expression for the fracture stress with an applied electric field:

$$
\sigma_{f}=\left(\frac{\varepsilon_{f}}{8 \pi}\right)\left(\frac{V E_{m} d}{2 F_{t}\left(1-v_{r}^{2}\right)}\right)^{2}
$$

Substituting the expression $F_{t}=\pi D^{2} \sigma_{f} / 4$ into equation (5.37) and rearranging gives

$$
\sigma_{f}=\left(\frac{\varepsilon_{f}}{8 \pi}\right)^{1 / 3}\left(\frac{2 V E_{m} d}{\pi D^{2}\left(1-v_{r}^{2}\right)}\right)^{2 / 3}
$$

which is the equation Kendall uses to compare to experimental data with reasonable success [31].

It is of interest to apply Kendall's model for the detachment force between two cylinders to the contact between a sphere and wall. By rearranging equation (5.38) and substituting the expression $\sigma_{\mathrm{f}}=4 \mathrm{~F} / \mathrm{t} \mathrm{D}^{2}$, an expression for the contact diameter $d$ is given by

$$
d=\left(\frac{32}{\varepsilon_{f}}\right)^{1 / 2}\left(\frac{2\left(1-v_{r}^{2}\right)}{E_{m} D V}\right) F_{t}^{3 / 2}
$$

Equation (5.39) can be used to calculate the contact diameter from the 
detachment force measured in the single contact experiments. This has been done here for one of the measurements shown in Fig. 5.10 for the $1.9 \mathrm{~mm}$ diameter spheres where a detachment force of $0.3 \times 10^{-3} \mathrm{~N}$ was measured for an applied voltage of $0.4 \times 10^{3} \mathrm{~V}$. The values of $v_{\mathrm{r}}$ and $\mathrm{E}_{\mathrm{m}}$ for lead glass are 0.25 and $6.2 \times 10^{10} \mathrm{~N} \mathrm{~m}^{-2}$, respectively. The value of $\varepsilon_{f}$ is $8.86 \mathrm{x}$ $10^{-12} \mathrm{~F} \mathrm{~m}^{-1}$ for air of $50 \%$ relative humidity [76]. For this case, the contact diameter given by equation (5.39) is $3.93 \times 10^{-10} \mathrm{~m}$. Such a small contact size is unreasonable, so it is clear that Kendall's model for cylinders where the electric field strength is uniform in the gap is not applicable to a spherical geometry with a highly non-uniform field distribution. However, Kendall's use of fracture mechanics theory to model the electrical clamping force at detachment is more realistic than a force balance approach for analysing the forces acting on deforming bodies, and should be extended to include a spherical geometry as part of future work in this field.

\subsection{ANALYSIS OF CONTACT RESISTANCE}

It was proposed in Chapter 4 that the assumption of Hertzian deformation of the contact area by the electrical clamping force was not obvious. Figure 5.21 shows that the magnitude of the electrical clamping force is very sensitive to the size of the contact. Here, the Fixed Cap model of equation (A4.31) from Appendix 4.1 has been used to show the dependency of the electrical clamping force on the contact radius, a. In order to assess whether the source of error in the electrical clamping force models is due to incorrect modelling of the contact size, the contact size needs to be measured with an applied electric field.

Optical microscopy and interferometry were initially investigated for measuring the contact size but were not pursued due to difficulties in configuring the experimental set-ups with an electric field [80]. Attempts were also made to measure the contact deformation due to the applied electric field, albeit not the contact area, with the Nanotest machine but this was also discontinued because the deformation of the apparatus, including the mounting of the glass spheres, was too large to accurately distinguish the deformation of the particle. However, the measurements of the electrical resistance of the spheres during the detachment experiments may provide an indirect method of inferring the size of the contact. 
The surface resistance over a sphere was given above in equation (5.8). By rearranging equation (5.8), an expression for $\theta_{0}$ is obtained as

$$
\theta_{0}=2 \cdot \tan ^{-1}\left(\tan \frac{\theta}{2} \exp \left(\frac{-2 \pi}{\rho_{s}} R_{\theta}\right)\right)
$$

The radius of the contact cap is calculated from $\theta_{0}$ by

$$
\mathrm{a}=\theta_{0} \cdot \mathrm{R}
$$

In order to calculate the size of the contact angle $\theta_{0}$ from the measurements of the resistance $R_{\theta}$ over the sphere, the values of $\theta$ and $\rho_{\mathrm{s}}$ are required in equation (5.40). The parameter $\theta$ is the position through which the resistance has been measured over the sphere, as defined in Fig. 5.6. For the mounted glass spheres used in the detachment experiments, the resistive surface of the sphere is limited by the edge of the Electrodag, as shown in Fig. 5.6. Therefore, the sphere's surface provides a resistance to the current flow from the angle $\theta_{0}$ to the angle $\theta$, where $\theta$ is calculated from the width of the Electrodag as

$$
\theta=\pi-\cos ^{-1}\left(\frac{L-R}{R}\right)
$$

The values of $\theta$ were calculated from measured values of $L$ and $R$ and were in the range $89^{\circ}-122^{\circ}$ for the 5 glass spheres used in the experiments.

The value of the surface resistivity of the sphere must also be known in order to calculate $\theta_{0}$ in equation (5.40). This parameter has proved to be difficult to evaluate. The glass spheres used in the single contact detachment experiments were prepared in the same way as the glass ballotini used in the arching and shear cell experiments discussed in Chapter 3. The spheres were taken as is from the supplier's container, dispersed into a $1 \%$ (by volume) solution of anionic surfactant in tap water for a few minutes, and then removed from the solution and left to dry. In the bulk experiments, this was done to ensure sufficient moisture adsorption on the surfaces of the particles so that surface conduction dominated over volume conduction, and to ensure fast charging and discharging so that time was not an additional variable in the experiments. The glass spheres in the single contact experiments were treated in the same way as the glass in the bulk experiments to provide comparable measurements of forces. This method 
of surface preparation for the glass spheres is unlikely to produce a highly uniform coating over the surface because of possible contamination sites on the glass surface before coating. Therefore, the exact quantification of the surface resistivity of the spheres was difficult.

An approximate value for the surface resistivity of the detergent coated glass spheres was obtained by measuring the surface resistivity of a coated glass sheet according to British Standard 6233:1982, 'Methods of test for volume resistivity and surface resistivity of solid electrical insulating materials'. The sheet was soda lime glass rather than lead glass as used in the single contact experiments because lead glass was not readily available in sheet form. However, the glass sheet was coated with surfactant similarly to the spheres and kept at $50 \%$ relative humidity so that surface conduction over the surfactant coating would dominate over conduction through the glass itself. Each piece of glass sheet was $120 \mathrm{~mm}$ square by $3 \mathrm{~mm}$ thick. As suggested by this method, annular electrodes formed from Electrodag were painted on the surface of the glass sheet as shown in Fig. 5.22, where the dimensions of the electrodes are such that $d_{1}$ $=50 \mathrm{~mm}, \mathrm{~d}_{2}=60 \mathrm{~mm}, \mathrm{~d}_{3}=80 \mathrm{~mm}$, and $\mathrm{d}_{4}=100 \mathrm{~mm}$. Brass backing plates were placed in good contact over the electrodes and the high voltage and earth wires were connected between the backing plates and the electrometer. The electric current, I, was measured between the outer ring electrode, which was the high voltage electrode and the inner electrode, which was earthed, for an applied voltage, $\mathrm{V}$, of $1 \mathrm{kV}$ after 1 minute. The third electrode on the bottom of the glass sheet acted as a guard electrode. The measurements were made with the glass sheet inside a screened box to minimize any stray currents from spurious external voltages. The surface resistivity was calculated as

$$
\rho_{\mathrm{s}}=\mathrm{R}_{\mathrm{a}} \frac{\mathrm{p}}{\mathrm{G}}
$$

where $\quad \mathrm{R}_{\mathrm{a}}=$ measured resistance across annulus $=\frac{\mathrm{V}}{\mathrm{I}}$

$G=$ gap between electrodes

$\mathrm{p}=$ average perimenter $=\pi\left(\mathrm{d}_{1}+\mathrm{G}\right)$

Surface resistivity was measured for two pieces of untreated glass and two pieces of coated glass which were coated in the same way as described above for the glass spheres. The temperature and relative humidity for the measurements were the same as for the single contact force measurements: 20 
$\pm 2{ }^{\circ} \mathrm{C}$ and $50 \pm 5 \%$. The average surface resistivity of the uncoated glass was $1.9 \times 10^{15} \pm 0.3 \times 10^{15} \Omega \mathrm{m} / \mathrm{m}$ and for the coated glass it was $1.5 \mathrm{x}$ $10^{12} \pm 0.2 \times 10^{12} \Omega \mathrm{m} / \mathrm{m}$. The surface resistivity measured for the coated glass sheet is of the same order of magnitude as the resistance measurements for the glass spheres shown in Fig. 5.11. From the ratio of $\mathrm{R}_{\theta} / \rho_{\mathrm{s}}$ in equation (5.8), it is expected that the surface resistivity of the glass spheres is of the same order of magnitude as the resistance of the spheres. Even for a very small value of $\theta_{0}$ of $0.001^{\circ}$ the ratio of $R_{\theta} / \rho_{s}$ is approximately 1.9 for $\theta$ ranging from $90-120^{\circ}$. However, differences in surface roughness and contamination, and chemical composition between the glass sheet and the spheres mean that the value of surface resistivity measured for the glass sheet cannot be considered to be a precise measurement of the surface resistivity of the spheres. In fact, work on the mechanisms of surface conduction by Jonscher [81] shows that the accurate measurement of surface resistivity for a particular surface is very difficult. Not only does his work point out the exponential relationship between surface resistivity and relative humidity, but it also shows that surface conduction over a dielectric is not uniform, but is instead filamentary. This puts in question the accuracy of using equation (5.8) to model the resistance over a sphere. Also, Jonscher finds that surface resistivity depends on the configuration of the electrodes used in the measurements, which puts doubt on the applicability of the glass sheet surface resistivity measurement to the case of the spheres where the electrodes' shape and size were very different.

Considering the above concerns over the accuracy of the measured value of $\rho_{\mathrm{s}}$, a sensitivity analysis was carried out to evaluate the effect of variations in $\rho_{\mathrm{s}}$ on the contact angle as predicted from equation (5.40). The results of the sensitivity analysis are shown in Fig. 5.23 where contact radii have been calculated from the contact angles using equation (5.41). The contact angles were calculated from the experimental measurements of contact resistance for the three spheres shown in Fig. 5.11(a) using the values of $\theta$ measured for each sphere. The contact angles were calculated for a range of values for $\rho_{\mathrm{s}}$ from 0.75 to $10.0 \mathrm{~T} \Omega \mathrm{m} / \mathrm{m}$ which includes the value of $1.5 T \Omega \mathrm{m} / \mathrm{m}$ measured from the glass sheet. The results for the contact radii are plotted in Fig. 5.23 versus the corresponding values of measured electrical force from Fig. 5.10. The results in Fig. 5.23 show that the value of contact radius predicted from the Sphere Resistance model using equations (5.40) and (5.41) is extremely 
sensitive to smail variations in $\rho_{\mathrm{s}}$. Fig. 5.23 also shows that the relationship between the contact radius and the electrical force is sensitive to the value of $\rho_{\mathrm{s}}$. For $\rho_{\mathrm{s}}=0.75 \mathrm{~T} \Omega \mathrm{m} / \mathrm{m}$, the contact radius changes significantly with changes in electrical force over the range of force measured in the experiments, whereas at higher values of $\rho_{\mathrm{s}}$ this sensitivity is greatly reduced. From the sensitivity analysis shown in Fig. 5.23, it is clear that it is not suitable to infer values of the contact size from calculations based on the Sphere Resistance model because of the difficulty in accurately measuring the surface resistivity.

In Fig. 5.23, the values of the contact radii calculated using the Sphere Resistance model are also compared to the contact radii which would result from Hertz theory. Equation (4.5) in Chapter 4 has been used to calculate the Hertzian contact radii for a glass sphere against a stainless steel plate using the measured values of the single contact electrical force. In this analysis using Hertz theory, it is assumed that the tensile force measured at detachment equals the compressive electrical force on the contact according to the force balance argument presented earlier in this chapter. Depending on the exact value of surface resistivity used to infer contact radius in the Sphere Resistance model, the Hertzian contact radii are either similar or highly dissimilar to the Sphere Resistance values. Therefore, further work on the measurement of the contact size with electric fields is needed to verify the validity of Hertzian deformation.

For the sake of completeness, a sensitivity analysis was also carried out to evaluate the effect of variations in $\theta$ on the contact angle as predicted from equation (5.40). The results of the sensitivity analysis are shown in Fig. 5.24 where contact radii have been calculated from the contact angles using equation (5.41). The contact angles were calculated from the experimental measurements of contact resistance for the $3.7 \mathrm{~mm}$ diameter sphere shown in Fig. 5.11(a) for $\rho_{\mathrm{s}}=1.5 \mathrm{~T} \Omega \mathrm{m} / \mathrm{m}$. The contact angles were calculated for a range of values for $\theta$ from $98^{\circ}$ to $138^{\circ}$ which includes the value of $118^{\circ}$ actually measured for the sphere. The results for the contact radii are plotted in Fig. 5.24 versus the corresponding values of measured electrical force from Fig. 5.10. The results in Fig. 5.24 show that the value of contact radius predicted from the Sphere Resistance model using equations $(5.40)$ and $(5.41)$ is not sensitive to small variations in $\theta$. This is expected since the resistance only changes significantly near the contact at small values of $\theta$ where the current is forced to flow through a 
small area.

In the above analysis using the Hertz theory, the process of fracture mechanics has been ignored since the measured detachment forces have been assumed to be equal to the equilibrium compressive forces acting on the contact. The fracture mechanics approach has been used by Johnson, Kendall and Roberts [72] to develop a modified version of the Hertz equation which takes into account adhesive/cohesive forces, like van der Waals forces, acting at a contact in addition to an externally applied force. In the well-known 'JKR' equation, the contact radius is given by

$$
a^{3}=\frac{R_{c}}{K_{c}}\left(F+3 \gamma_{s} \pi R_{c}+{\sqrt{\left(6 \gamma_{s} \pi R_{c} F\right)+\left(3 \gamma_{s} \pi R_{c}\right)}}^{2}\right)
$$

where $\gamma_{\mathrm{s}}$ is the energy per unit contact area. Using the subscripts 1 and 2 to refer to the sphere and the wall respectively, $R_{c}$ and $K_{c}$ are defined as

$$
\begin{gathered}
R_{c}=\frac{R_{1} R_{2}}{R_{1}+R_{2}} ; R_{c}=R_{1} \text { for contact between a sphere and a wall } \\
K_{c}=\frac{4}{3 \pi\left(k_{c 1}+k_{c 2}\right)}
\end{gathered}
$$

where $\mathrm{k}_{\mathrm{c}}=\frac{1-v_{r}^{2}}{\pi \mathrm{E}_{\mathrm{m}}}$

The adhesive forces modelled in the JKR equation are surface forces and in this way they are similar to the electrical clamping forces. It is therefore interesting to explore whether the effect of the electrical clamping force on the contact size can be described in terms of an equivalent surface energy acting at the contact. It should be noted that there is an important difference between the JKR model for the adhesive forces and the electrical clamping forces. In the JKR model, the adhesive forces are considered to act only over the contact area and any contributions in the surrounding gap are ignored. The electrical clamping forces act in the gap surrounding the contact, so there is an obvious conflict with the JKR model. On the other hand, the adhesion model of Derjaguin, Muller and Toporov (the DMT model) [82] takes into account the forces acting on the surfaces in the gap surrounding the contact in determining the adhesive force at the contact, but it does not consider that this force changes the contact area from the Hertzian value. The 
measurements of sphere resistance in the single contact experiments indicate that the contact area is changing with increased electric field strength. In view of this, the DMT model is not applicable for describing the effect of the electrical clamping force on the contact area, and the JKR model is used here to describe the effect of the electric field on the contact area in terms of an equivalent surface energy. This surface energy is not constant, as in the JKR case, but is dependent on the electric field strength. As the applied load is made negative in equation (5.44) to describe a tensile force, the contact radius decreases. For a real solution to be obtained for equation (5.44), the value under the square root should be positive, hence:

$$
F \geq-\frac{3}{2} \gamma_{s} \pi R_{c}
$$

Therefore, separation of the spheres will just occur when

$$
F_{s}=-\frac{3}{2} \gamma_{s} \pi R_{c}
$$

Setting equation (5.48) into equation (5.44) gives the value of the contact radius at separation as

$$
a=\left(\frac{3}{2} \gamma_{s} \pi \frac{R_{c}^{2}}{K_{c}}\right)^{1 / 3}
$$

It was found above that the detachment forces measured in the single contact experiments can be described very well as a linear function of the applied electric field strength such that

$$
F_{e}=C_{1} E_{a}
$$

where $C_{1}$ is obtained by fitting the data to equation (5.50). By setting the JKR detachment force, $F_{\mathrm{s}}$, from equation (5.48) equal to the electrical detachment force, $\mathrm{F}_{\mathrm{e}}$, from equation (5.50) (omitting the negative sign because $F_{e}$ is inherently tensile), an expression for the surface energy as a function of electric field is found as

$$
\gamma_{\mathrm{s}}=\mathrm{C}_{2} \mathrm{E}_{\mathrm{a}}
$$

where $C_{2}=\frac{2 C_{1}}{3 \pi R_{c}}$ 
Equation (5.51) can now be substituted into equation (5.49) to obtain an expression for the contact radius at detachment as a function of the applied electric field as

$$
a=\left(\frac{3}{2} \pi \frac{R_{c}^{2}}{K_{c}} C_{2} E_{a}\right)^{1 / 3}
$$

Combining equations (5.52) and (5.41) gives an expression for the contact angle as

$$
\theta_{0}=C_{3} E_{a}^{1 / 3}
$$

where $C_{3}=\left(\frac{3 \pi C_{2}}{2 R_{c} K_{c}}\right)^{1 / 3}$

The expression for $\theta_{0}$ given by equation (5.53) can now be substituted into the expression for the resistance of a sphere given by equation (5.8) to obtain a relationship for the sphere resistance as a function of the applied electric field as

$$
\mathrm{R}_{\theta}=\frac{\rho_{\mathrm{s}}}{2 \pi} \ln \left(\tan \frac{\theta}{2}\right)-\frac{\rho_{\mathrm{s}}}{2 \pi} \ln \left(\tan \left(\mathrm{C}_{4} \mathrm{E}_{\mathrm{a}}{ }^{1 / 3}\right)\right)
$$

where $C_{4}=\left(\frac{C_{1}}{8 R_{c}^{2} K_{c}}\right)^{1 / 3}$

Equation (5.54) can be expressed in the form of a linear equation to simplify fitting the experimental data for $\mathrm{R}_{\theta}$ and $\mathrm{E}_{\mathrm{a}}$ such that

$$
\mathrm{Y}=\mathrm{mX}+\mathrm{b}
$$

where $\quad \mathrm{Y}=\mathrm{R}_{\boldsymbol{\theta}}$

$$
\begin{aligned}
& \mathrm{X}=\ln \left(\tan \left(\mathrm{C}_{4} \mathrm{E}_{\mathrm{a}}{ }^{1 / 3}\right)\right) \\
& \mathrm{m}=-\frac{\rho_{\mathrm{s}}}{2 \pi} \\
& \mathrm{b}=\frac{\rho_{\mathrm{s}}}{2 \pi} \ln \left(\tan \frac{\theta}{2}\right)
\end{aligned}
$$


Equation (5.55) has been fit to the experimental data and it is found that the shape of the resistance versus electric field curves can be described well by it, as shown in Fig. 5.25 for one of the spheres. However, the validity of using equation (5.55) to describe the effect of electric field on contact resistance is in doubt because the values of $\rho_{\mathrm{s}}$ and $\theta$ obtained from the best fit values of $m$ and $b$ for each sphere do not agree with the measured values. The best fit values of $\rho_{\mathrm{s}}$ and $\theta$ are listed in Table 5.2 with the measured values. As described above, the measured value of $\theta$ was determined by determining the extent of the Electrodag coverage of the sphere under a microscope. The measured value of $\rho_{\mathrm{s}}$ was obtained from the glass sheet. The best fit values of $\rho_{\mathrm{s}}$ are one to two orders of magnitude too large and the values of $\theta$ are one to two orders of magnitude too small. Even if the value of $\rho_{\mathrm{s}}$ is varied in equation (5.55), the best fit values of $\theta$ are still much too small, which suggests that the model is not correct.

\subsection{CONCLUSIONS}

Measurements of the electrical clamping force at a single contact were presented here for the first time. The detachment forces for contacts between glass spheres of 1.9 and $3.7 \mathrm{~mm}$ diameter and polished stainless steel plate with electric fields were measured for comparison with the predictions of the electrical clamping force models. The force increased with increasing size of the particles as predicted, but the actual magnitude of the forces was incorrectly predicted by the theories. As was expected from the analysis of the macroscopic behaviour in Chapter 4, the Elastic Cap model overpredicted the single contact force and the Limited Field model underpredicted the single contact force. These measurements confirm that the electrical clamping force models are invalid. It was proposed in Chapter 4 that the error in the models may be caused by assuming Hertzian deformation of the material by the electrical clamping force. In order to confirm this hypothesis and to correctly model the deformation pattern, data is needed for the change in contact size with applied electric field.

In the single contact measurements, electric current over the sphere was measured as a method of determining the point of detachment. The current 
measurements were also used to calculate the electrical resistances of the spheres. The sphere resistance decreased with increasing electric field, which indicates that the contact area was increasing. Using estimated values for the surface resistivity of the spheres, the contact radii were calculated using the Sphere Resistance model. The contact radii which were calculated in this way are highly sensitive to small changes in surface resistivity. The surface resistivity of the spheres cannot be accurately measured. Therefore, this method is not considered suitable to infer values of contact radii with electric fields. Therefore, the values of contact radius calculated from the Sphere Resistance model could not be used to evaluate the validity of Hertzian deformation with electric fields.

An attempt was made to model the resistance by assuming that the contact area increased according to an effective surface energy which was a function of the electric field. This model was adapted from the 'JKR' model which describes the non-Hertzian contact area formed between surfaces due to adhesive forces. The predictions of sphere resistance from the model follow the experimental data well but the best fit values of surface resistivity and sphere resistance angle from the model do not agree with the experimentally estimated values.

Attempts made here to model the effect of electric fields on contact area have been unsuccessful. The Sphere Resistance model is not suitable for inferring values of contact area because of the sensitivity to surface resistivity which is very difficult to measure accurately for the particles involved in the experiments. Therefore, it is recommended that an experimental programme is continued with the aim of measuring the contact size directly. Methods which might be developed are direct observation by ordinary microscope of very large bodies, light interference fringe analysis, e.g. Newton's rings, of large bodies [80], and confocal laser microscopy which can measure changes in topography as small as $50 \mathrm{~nm}$. 


\section{CHAPTER 6}

\section{CONCLUSIONS}

This research was initiated by the invention of the Electromechanical Valve for Solids (EVS), where a DC electric field is applied to to a packed bed of particulate solids to control the flow. The EVS provides several advantages over the mechanical devices traditionally used in industry for flow control of granular materials, in particular its fast response and reduced wear characteristics. In order to develop the device to its full potential and optimise its performance, a model for flow was required. This required a greater understanding of the fundamental mechanisms of its operation, in particular, an understanding of the interactions between the electrical and mechanical forces acting on the particles.

When an electric field is applied to a packed bed of particulate solids, several types of electrical force may be generated. In the case of the EVS, two key aspects of its operation provide evidence for the dominating electrical force which is responsible for the effect on flow: the particles must remain in continuous contact; a small current must be conducted through the bed, i.e. the particles must not be highly insulating. These characteristics have also been identified as critical to the behaviour of electrofluidised beds and electrostatic precipitator cakes. There, the dominating electrical force mechanism has been attributed to the electrical clamping force. These forces are caused by the constriction of current at contact points between the particles, and between the particles and the electrodes, which results in greatly enhanced electric fields and the generation of forces across the surfaces of the particles in the gap surrounding the contact area. In the EVS, electrofluidised beds, and electrostatic precipitator cakes, the observed behaviour cannot be explained by recourse to other electrical force mechanisms, i.e. the force acting on the bed due to the electrostatic force of attraction between the electrodes or the polarization force between the particles. From this evidence, the effect of the electric field on flow in the EVS must be due to the electrical clamping forces. Therefore, the specific objective of this research was to develop a qualitative and quantitative understanding of the effect of the electrical clamping forces on the mechanics of granular materials. The main results and conclusions of the research are summarised below. 
A review of the previous investigations of the electrical clamping force showed that these forces significantly alter the behaviour of packed beds of particulate solids. All the experimental measurements have been of bulk behaviour with applied DC electric fields. In particular, McLean [15] measured the tensile stresses required to detach a plate from a cake of fly ash. Dietz and Melcher [20,39] carried out experiments to characterize bulk behaviour of electrofluidised beds. These included measuring pressure drop in an electrofluidised bed, measuring the gas flowrate required to suspend a bed of particles, and measuring shear stresses in a Couette viscometer. Robinson and Jones [32] measured the yield stresses between a plate and bed of particles. In all cases, the bulk materials exhibited increasing resistance to failure with increasing electric field strength.

In some of the previous work, theoretical models for the electrical clamping force have been developed. These models are for the force at a single contact and are mostly for sphere/sphere or sphere/wall contacts. Recently, Kendall [31] developed a model for the electrical clamping force which is specific to the geometry of an annular crack in a cylinder. As spherical particles were used in this work, Kendall's model was not directly applicable to the analyses. Therefore, the sphere models were considered for the most part in this work.

No direct measurements have previously been made of the electrical clamping force at a single contact to verify the models. Also, most of the investigations do not attempt to compare bulk experimental data with predictions based on the models. In order to do this, it is necessary to transform the single contact force models into a macroscopic representation of the bulk stresses in the material. Dietz [19] and Dietz and Melcher $[20,39]$ attempted this transformation but their method is flawed. Therefore, the first part of this research focused on developing a formulation to describe the bulk mechanics of granular materials using the electrical clamping force models for a single contact.

Experiments were carried out to characterize three aspects of flow in the EVS: initiation and halting of flow, steady-state flow, transient flow. The experiments were intended to provide qualitative guidance for modelling and quantitative results by which to assess the models. The first two aspects of flow were later analysed in detail using the electrical clamping force models. A quantitative analysis was not carried out on transient 
flow.

First, the initiation and halting of flow were examined experimentally. Two types of experiments were carried out using simple electrode systems to produce well-defined electric fields. Glass ballotini $(600-710 \mu \mathrm{m})$ were used as a model spherical, cohesionless material. In one experiment, the effect of an electric field on internal shear stresses at failure was measured using a box-type shear cell where the bottom and top surfaces of the box formed the electrodes. Yield loci were measured over a mechanical applied load range of $0.4-3.7 \mathrm{kN} \mathrm{m}^{-2}$ for three applied field strengths: 0,470 and $800 \mathrm{kV} \mathrm{m}^{-1}$. The application of the field resulted in increasing yield stresses with increasing electric field. With and without an applied field, the yield loci followed straight lines and thus, could be described by the Coulomb yield function. The electric field caused a vertical shift in the yield loci approximately parallel to the zero field locus. The yield loci measured by Robinson and Jones [32] for wall failure with electric fields showed a similar influence of electric field.

Using the Coulomb yield function to model the internal and wall yield loci, the effect of the electric field could be described either as producing an additional external stress on the material, $\sigma_{e}$, or as producing cohesion/adhesion in the material, $c_{e}$. Mathematically, either interpretation was shown to describe the experimental data equally well. Values of $\sigma_{e}$ and $c_{e}$ were fit to the experimental data for internal yield and wall yield.

In the second experiment on the initiation and halting of flow, a wedge-shaped hopper with electrodes forming the side walls was used to evaluate the formation of a stable arch by application of an electric field. The macroscopic process of arching is relevant to the EVS where halting of the flow is observed to occur by formation of stable arches over the mesh openings of the downstream electrode. In the arching experiments, the static holding voltages were measured for varying orifice width and hopper half angle, and for two electrode wall roughnesses. The holding voltage is the minimum voltage required to hold the material which is already in a static state. The holding voltages increased with increasing orifice width and decreased with increasing wall roughness. There was little dependence shown on hopper half angle. 
Interesting behaviour in the arching experiments was also shown by the measurement of dynamic holding voltages. These were measured by allowing the material to flow with no applied voltage and then applying a voltage to stop the flow. The dynamic holding voltages were always higher than the static holding voltages for the same hopper configuration. This may be due to decreased contact density for material in a dilated state or to sliding contacts near the walls affecting the electrical clamping forces.

It was also noted that the application of very high voltages to material in a static state, followed by lowering of the voltage, resulted in failure occurring at a voltage lower than static holding voltage. This shows that higher electric fields produce more stable structures than lower ones, even after reducing the field. This effect may be due to contact deformation resulting in enhanced van der Waal's forces or modified contact friction resistance.

The second aspect of flow in the EVS to be examined was steady-state flow. Performance curves for flowrate as a function of electric field strength were measured for a variety of materials, including glass ballotini, sand, and $\mathrm{NaCl}$ salt, in cylindrical and rectangular configurations of the EVS. These results showed that flowrate in the EVS decreases with increasing field strength. The flowrate depended on the average electric field strength and on the distribution of the electric field between the electrodes. Using a steady applied electric field, the EVS was used successfully to halt the flow of a granular material and to control the flowrate in the upper half of the full flowrate range.

Transient flow was the third aspect of flow in the EVS which was investigated. The dynamic response of flow to changing electric field strength was examined in three experiments. First, flowrates were measured for pulsating fields as a function of pulse width and frequency. The flowrate was found to decrease with increasing pulse width and increasing frequency. This behaviour is due to the charging and discharging of the EHT capacitor and the material between the electrodes in the EVS. In the pulsating mode, good control of flowrate is obtained for the lower half of the full flowrate range. Therefore, the steady and pulsating field modes of operation complement each other to provide an overall wide turndown ratio. 
Attempts were made to measure the time constant of the EVS with changing electric field for a voltage step change. As the charging and discharging time of the particles is of the order of milliseconds, it was the response time of the individual particle movement which was of interest here. These experiments were unsuccessful because the response of the system was dominated by the response time of the weighing device which was used to monitor the change in flowrate, approximately $0.2 \mathrm{~s}$.

The deficiencies of the weighing devices were emphasised further in attempts to evaluate the response of the EVS to a PID control programme in a computerised closed loop system. The EVS produced good flowrate control with respect to final setpoint error, but the fast response of the EVS could not be exploited due to the long stabilisation time of the weighing device.

Detailed analysis of the experimental results for macroscopic flow behaviour with electric fields focused on the results of the yield and arching experiments. In both cases, formulations were developed for translating the single contact electrical clamping force models into representations of bulk electrical stress. The electrical stress was then superimposed on the mechanical stresses in the material as an additional external stress on the material.

This formulation was most straight-forward for the case of wall failure where the size and direction of the shear zone is well-defined. The yield loci measured by Robinson and Jones [32] were compared to the theoretical predictions using the four models of the electrical clamping force. Three of the models significantly overpredicted the contribution of the electrical clamping force by two orders of magnitude. These models were the Fixed Cap, Elastic Cap and Combined Force models.

The electrical stresses predicted by the Fixed Cap model were unrealistic because the yield stress was predicted to decrease with increasing normal mechanical load, which is opposite to the measured effect. The unrealistic response of the Fixed Cap model for predicting wall yield stresses indicated that it is incorrect to ignore the contribution of the electrical clamping force to contact deformation. The Fixed Cap model was not evaluated further due to its obvious unsuitability. 
The electrical clamping stresses predicted by the Elastic Cap and Combined Force models were smaller than those predicted by the Fixed Cap model because the magnitude of the electrical clamping forces were predicted such that they produced greater contact deformation than the mechanical forces. In fact, the yield loci predicted by the Elastic Cap and Combined Force models indicated that the deformation caused by the electrical clamping forces dominated over that caused by the mechanical forces. Therefore, the magnitude of the electrical clamping forces was nearly independent of the magnitude of the mechanical forces. The measured yield loci also showed that the electrical clamping forces were independent of the mechanical forces since the yield loci with applied fields were shifted parallel to the zero field loci. However, the magnitude of the increase in shear caused by the electrical clamping stress was not predicted correctly by the models, being two orders of magnitude too large.

The yield stress predictions of the fourth electrical clamping force model, the Limited Field model, were one order of magnitude too small and produced negligible shift of the yield loci from the zero field case. This model assumes that electric breakdown occurs at the contact points between particles thus limiting the maximum achievable field. The gap heights outside the particles in this work are considered to be sufficiently small to support much higher breakdown field strengths than normally supported in air. Also, measurements of the current voltage relationship in this work show Ohmic behaviour. Therefore, the Limited Field model was not considered to be relevant to describe the experimental work in this research.

In order to evaluate the internal yield data with the electrical clamping force models, a formulation was developed for the average electrical clamping stress in the material by assuming that the probability of contacts between particles was uniformly distributed over the surfaces. In this way, the directional dependence of the electrical clamping force on applied electric field was accounted for approximately.

The analysis of the internal yield data with the predicted electrical clamping stresses superimposed on the mechanical stresses confirmed the results of the wall yield analysis. The Elastic Cap and Combined Force models predicted stresses one order of magnitude too large, and the Limited Field model predicted stresses one to two orders of magnitude too small. 
The results of the arching experiment were analysed using two approaches to describe the interaction of the electrical clamping forces and the mechanical forces. The first approach to modelling the arch holding voltage followed the approach used to model the yield stresses, i.e. the electrical clamping forces were assumed to act as an additional applied stress on the material. The electrical clamping stress on the wall was incorporated into a force balance over an arched differential slice in the hopper. By solving the force balance for the condition of zero normal stress on the free surface of an arch, a predictive model for the holding voltage was obtained. The second approach used to analyse the arch experiment modelled the effect of the electrical clamping force as a cohesion in the material. The cohesion in the material was described quantitatively in terms of the unconfined yield stress. A relationship for unconfined yield stress as a function of applied field was fit from the experimental data from the internal yield experiments. The force balance over the arched element was solved in this case for a material with an unconfined yield stress described by this measured relationship. In this way, a second model was obtained to predict the arch holding voltage.

With respect to an assessment of the validity of the electrical clamping force models and their physical interpretation, the results of the arching analysis were inconclusive. Both the external stress approach with the Elastic Cap model for the electrical clamping force and the cohesion approach gave very similar agreement with experiment. The first model was based on a theoretical model of the electrical clamping force which was found to give huge discrepancies between theory and experiment in the shear cell analysis. The second arch model was based on real shear cell experimental data. The similarity between the results from the alternative models shows that the stress analyses developed for the hopper are not sensitive to the electrical clamping forces and thus, are not good methods for evaluation.

An analysis of steady-state flow in the EVS was presented using a semi-empirical approach to describe the effect of the electrical clamping forces by analogy to the retarding effect of interstitial gas pressure gradients on discharge from hoppers [33]. The functional form of the flow equation fit the experimental data well, and could be used for calibrating the performance of the EVS for practical operation. However, constants in 
the model which were fit from the experimental data showed a wide range of values. Therefore, the fundamental approach in the model was not correct. In particular, the effect of electric field distribution was not accounted for. Experiments showed that different field distributions produced different flowrates for the same average applied field.

The analyses of the bulk experimental data failed to resolve two important issues concerning the electrical clamping forces. First, the validity of the electrical clamping force models themselves was put into question by the analyses of yield stresses, where the theoretical predictions from the models differed significantly from the experimental results. The analyses of arching and steady-state flow were inconclusive concerning the validity of the electrical clamping force models. Second, the nature of the macroscopic representation of the electrical clamping effect as an external applied force or cohesion was unresolved. Mathematically, either approach could be used to describe the yield stress and arching results equally well. Physically, cohesion between particles must exist at the surface of a stable arch. However in the arching experiments performed here, it is possible that other electrical forces are produced by the high electric field gradient at the arch surface. It was speculated that these forces, i.e. dielectrophoretic forces, and not the electrical clamping forces, may be responsible for supporting the stable surface of the arch. However, these forces are difficult to evaluate theoretically [68], and the experimental evidence was not conclusive. In view of these discrepancies and difficulties with the existing models, it was decided to make a direct experimental evaluation of the electrical clamping force.

The electrical clamping forces at single contacts were measured here for the first time. The measurements were made by modifying a Nanotest device which was normally used for nanoindentation work. The electrical clamping forces were measured for the detachment of glass spheres of 1.9 and $3.7 \mathrm{~mm}$ diameter from contact with a very smooth stainless steel surface. The results were compared to the electrical clamping force models. The force increased with increasing particle diameter, as predicted. However, the magnitude of the forces was incorrectly predicted by the theories. The Elastic Cap model overpredicted the single contact force, and the Limited Field model underpredicted the single contact force. The single contact force measurements corroborated the results of the macroscopic yield stress analyses, and confirmed that the electrical clamping force models are 
invalid.

It was speculated that the error in the models may be due to the incorrect assumption of Hertzian deformation at the contact. An attempt was made to quantify the contact size from measurements of electrical resistance using the model for contact resistance of a sphere. These calculations showed that the contact size was highly sensitive to the surface resistivity of the sphere, which cannot be measured accurately. Therefore, the validity of Hertzian deformation with applied electric fields could not be evaluated using the approach.

Finally, a model for the contact area with electrical clamping forces was developed based on the JKR model for the non-Hertzian contact area caused by adhesive forces. In the model, the contact area is assumed to increase according to an effective surface energy which is a function of the electric field. The model for contact area was used with the model for sphere resistance to analyse the experimental data for resistance. The theoretical predictions could be made to follow the experimental data well, by fitting the model constants to the experimental data. However, the best fit values of the constants did not agree with the measured values.

The analyses of contact size show that it is difficult to accurately infer the contact size from theory because of the sensitivity to surface resistivity of the particle, which is difficult to measure. These results indicate that direct measurement of the contact size is necessary in order to ascertain the deformation caused by the electrical clamping force. 


\section{CHAPTER 7}

\section{RECOMMENDATIONS FOR FUTURE WORK}

This research has laid a firm foundation for the further characterization of the electrical clamping force at a single contact. The research has shown that the single contact models of the electrical clamping force are invalid, and moreover, that the fundamental mechanism of the electrical clamping force is in doubt, i.e. external force or cohesion. Therefore, it is important that further work in this field first resolves these issues for single contact behaviour. This knowledge can then be used to build reliable models of bulk behaviour of particulate solids in electric fields for use in industrial applications such as electrostatic precipitation and filtration, ER fluids, electrostatic compaction, and the EVS.

\section{Single Contact Evaluation}

In the first instance, the systematic experimental evaluation initiated in this work should be continued for the measurement of the electrical clamping force at a single contact. In the first instance, the objective here would be to establish the general relationships for the electrical clamping force as a function of the relevant variables (e.g. electric field, particle size, particle roughness, surface resistivity) under different loading conditions by building up a database of experimental measurements. This information could then be used in assembly calculations of bulk assemblies and for developing a new fundamentally sound model for the electrical clamping force.

Single contact measurements should initially be made for particles with very smooth surfaces with highly uniform surface resistivities. For instance, sapphire and other minerals are available as spheres with very smooth surfaces (e.g. less than $1 \mu \mathrm{m}$ asperity height) [75]. The surface of the particles should be made as clean as possible, such as by high temperature heating or solvent cleaning, to remove surface contamination. This should also allow for very ever coating of the surface if a surface coating is then applied in a controlled manner. The control and modification of surface resistivity is of interest in order to determine the relationship between the magnitude of surface resistivity and the electrical clamping forces. Although the previous single contact models were developed based on either surface or volume conduction, the models 
themselves do not contain surface or volume resistivity as a variable. However, experimental observations with different materials at different relative humidities indicate that there is an effect.

The single contact measurements in this work were made under normal (tensile) loading conditions. In addition to continuing these measurements, it is necessary to evaluate the electrical clamping force in friction. To accomplish this, measurements of single contact friction with varying mechanical loading of the contact and varying electric field strength can be made with the Nanotest device which has a facility for mounting a friction transducer on the pendulum. It needs to be resolved whether the electrical clamping forces simply act as additional external body forces, or, whether they modify the adhesive nature of the solids without affecting the prevailing mechanical forces. Recently, Israelachvili et al. [83] have found that, for surfaces where the formation and breaking of an adhesive contact is reversible, there is no effect on friction by the adhesive force. It could be expected that the formation and breaking of a contact with an electric field would be reversible, in which case the electrical clamping forces would not affect the friction forces at the contact. This is a highly interesting concept and should be investigated. An understanding of this fundamental nature of the electrical clamping forces will enable a realistic single contact model to be developed, and is crucial for valid modelling of bulk behaviour where the existence of stable free surfaces must be explained.

Further work on single contact measurements of detachment forces and friction forces should be carried out for various systems of particles and surfaces to build up a base of knowledge and data for modelling particulate systems. As mentioned above, experiments should start with well-defined systems of single contacts between very smooth surfaces. This includes contacts between a sphere and a wall, and also contacts between two particles. The effects of particle shape, surface roughness, natural cohesion, and surface resistivity can then be investigated at the single contact level by selecting suitable materials.

In order to develop a new fundamental model for the electrical clamping force, it is necessary to couple knowledge of the contact deformation with the resulting force. An attempt was made in this work to analyse the contact size by inference from data for the electrical resistance of the 
contact. Also, attempts were made to measure the deformation of the particles with the Nanotest device but this was unsuccessful due to the dominance of the instrument compliance. Even if it were possible to measure the deformation of the particle normal to the contact with the Nanotest, assumptions would have to be made in order to relate this deformation to the deformation of the particle surface near the contact and to the contact size itself. Both pieces of information are needed to describe the electric field distribution and the resulting force distribution in the gap outside the contact area. If possible, the shape of the surface near the contact and the contact size should be measured directly to avoid assumptions in the modelling. While the shape of the surface is particularly difficult to quantify, it may be possible to measure the contact size by analysis of Newton's rings (i.e. interference fringes) which are generated by contact between a large sphere and a glass plate rendered conductive with indium tin oxide coating. It may also be possible to measure the contact size using confocal laser microscopy where the contact between a sphere and a conductive glass plate is scanned normally to the contact for variations in surface topography.

The experimental programme described above should provide fundamental evidence for the development of a new correct model for the electrical clamping force. In future work on modelling, it is important that a Fracture Mechanics approach is adopted, as used by Kendall [31]. This method takes into account the balance of strain energy and the work of the electric field. In a deforming system, it is clearly incorrect to simply use a Force Balance approach which ignores energy changes.

\section{Macroscopic Evaluation}

The ultimate objective of this work is to evaluate the bulk behaviour of granular materials with applied electric fields. To accomplish this, a methodology is needed to calculate the distribution of the single contact forces in an assembly. This can be approached using assembly calculations, where the mechanical force network through the packing can be calculated, followed by the calculation of current paths. With the current paths established, the local electric field at each contact can be calculated. From the experimental characterization of the single contact electrical clamping forces, it should now be possible to incorporate these forces into the force network. 
Experiments need to be carried out to verify the methodology which is developed for the net stresses in particulate assemblies. As for the single contact experiments, this work should be carried out systematically, starting first with a monolayer of smooth, spheres between two flat plate electrodes where detachment force and shear forces can be measured and analysed. More complex assemblies can then be designed to evaluate the effect of electrode geometry, particle shape, and packing order. Finally, it should be possible to analyse the macroscopic bulk behaviour in complex systems, in particular the EVS.

The most complex aspect of flow in the EVS is the dynamic behaviour with changing electric fields. Attempts to analyse the response time of the material by measuring changes in bulk flowrate were unsuccessful due to the slow response of the load cells. Further work could be carried out using a high speed video camera and image analysis software to monitor the movement of particles between the electrodes in the EVS. 


\section{REFERENCES}

1 A.G. Bailey, Electrostatic phenomena during powder handling, Powder Technology, 37 (1984) 71 .

2 K.P. Homewood, Do 'dirty' surfaces matter in contact electrification experiments?, J. Electrostatics, 10 (1981) 299.

3 K.P. Homewood and A.C. Rose-Innes, An investigation of contact-charge accumulation on insulators repeatedly touched by metals, J. Phys. D: Appl. Phys, 15 (1982) 2283.

B.N. Cole, M.R. Baum and F.R. Mobbs, An investigation of electrostatic charging effects in high-speed gas-solids pipe flows, Proc. Instr. Mech. Engrs., 184, 3C (1969-70) 77.

5 S. Joseph and G.E. Klinzing, Vertical gas-solid transition flow with electrostatics, Powder Technology, 36 (1983) 79.

6 R. Kuczynski, A. Przekwas and W. Kucinski, The static electrification of particles in gas-solids pipe flow, J. Electrostatics, 10 (1981) 309.

7 P.J. Larouere, S. Joseph and G.E. Klinzing, Some stability concepts in relation to electrostatics and pneumatic transport, Powder Technology, 38 (1984) 1.

E.E. Smeltzer, M.L. Weaver and G.E. Klinzing, Individual electrostatic particle interaction in pneumatic transport, Powder Technology, 33 (1982) 31.

9 J. Böhm, Electrostatic Precipitators, Elsevier, Amsterdam, (1982).

10 W. Strauss, Industrial Gas Cleaning, 2nd ed., Pergamon Press, Oxford, (1975).

11 M. Ghadiri, J.A.S. Cleaver and R. Seaton, Electrically enhanced gas filtration using microsieves as filter media, 1st Eur. Symp. Sep. Part. Gases, PARTEC, Nürnberg, 19-21 April 1989, Nürnberg Messe, Nürnberg, (1989) 245.

12 G.W. Penney, Using electrostatic forces to reduce pressure drop in fabric filters, Powder Technology, 18 (1977) 111.

13 E. Schmidt, Elektrische Beeinflüssing der Partikelabscheidung in Oberflächenfiltern, Dissertation for Dr.Ing., Universität Karlsruhe, Karlsruhe, (1991).

14 H.A. Pohl, Dielectrophoresis, Cambridge University Press, Cambridge, (1978).

15 K.J. McLean, Cohesion of precipitated dust layer in electrostatic precipitators, J. Air Pollution Control Assoc., 27, 11 (1977) 1100.

16 G.B. Moslehi and S.A. Self, Electromechanics of precipitated particulate layers, IEEE Trans. Ind. Appl., IA-20, 6 (1984) 1598. 
17 G.M. Colver, Bubble control in gas-fluidised beds with applied electric fields, Powder Technology, 17 (1977) 9.

18 T.W. Johnson and J.R. Melcher, Electromechanics of electrofluidised beds, Ind. Eng. Chem. Fundam., 14, 3 (1975) 146.

19 P.W. Dietz, Electrofluidized Bed Mechanics, Ph.D. Thesis, M.I.T., Cambridge, Mass., (1976).

20 P.W. Dietz and J.R. Melcher, Momentum transfer in electrofluidised beds, AIChE. Symp. Ser., 74, 175 (1978) 166.

21 H. Block and J.P. Kelly, Electro-rheology, J. Phys. D.: Appl. Phys., 21 (1988) 1661.

22 R.T. Bonnecaze and J.F. Brady, Dynamic simulation of an electrorheological fluid, J. Chem. Phys., 96, 3 (1992) 2183.

23 W.M. Winslow, Induced fibration of suspensions, J. Appl. Phys., 20 (1949) 1137.

24 M. Ghadiri and R. Clift, Eur. Pat. Appl. No. 87308304.2, (1987).

25 M. Ghadiri, C.M. Martin, J.E.P. Morgan and R. Clift, An electromechanical valve for solids, Powder Technology, 73 (1992) 21.

26 C.M. Martin and M. Ghadiri, Evaluation of a flow control system for granular materials using the electromechanical valve for solids, BULK 2000, London, 29-31 October 1991, Proc. IMechE., 12 (1991) 131.

27 D. Geldart and P. Jones, The behaviour of L-valves with granular materials, Powder Technology, 67 (1991) 163.

28 T.M. Knowlton, Solids transfer in fluidized systems, in Gas Fluidization, ed. D.G. Geldart, Wiley, Chichester, (1986) 341.

29 L.S. Leung, Y.O. Chong and J. Lottes, Operation of V valves for gas-solid flow, Powder Technology, 49 (1987) 271.

30 E. Jaraiz-M., O. Levenspiel and T.J. Fitzgerald, The uses of magnetic fields in the processing of solids, Chem. Eng. Sci., 38, 1 (1983) 107.

31 K. Kendall, Adhesive effect of electric fields across cracks, J. Phys. D: Appl. Phys., 24 (1991) 1072.

32 K.S. Robinson and T.B. Jones, Particle-wall adhesion in electropacked beds, IEEE Trans. Ind. Appl., IA-20, 6 (1984) 1573.

33 R.M. Nedderman, U. Tüzün and R.B. Thorpe, The effect of interstitial air pressure gradients on the discharge from bins, Powder Technology, 35 (1983) 69.

34 H.M. Pollock, D. Maugis and M. Barquins, Characterization of submicrometre surface layers by indentation, in Microindentation Techniques in Materials Science and Engineering, ASTM STP 889, P.J Blau and B.R. Lawn eds., Philadelphia (1986) 47.

R. Holm, Electric Contacts, 4th ed., Springer-Verlag, Berlin, (1967). 
A.D. Stuckes, Some theoretical and practical considerations of the Johnson-Rahbek effect, Proc. Inst. Elect. Eng. Lond., 103 (1956) 125.

37 J. Bares, Electrical conductivity of packed particle beds, IEEE Trans. Ind. Appl., 24, 6 (1988) 1050.

K. Kendall, Electrical conductivity of ceramic powders and pigments, Powder Technology, 62 (1990) 147.

39 P.W. Dietz and J.R. Melcher, Interparticle electrical forces in packed and fluidised beds, Ind. Eng. Chem. Fundam., 17, 1 (1978) 28.

40 P.J. Buysman and G.A.L. Peersman, Stability of ceilings in a fluidized bed, Proc. Int. Symp. on Fluidization, Eindhoven, Netherlands University Press, (1967) 38.

41 J.P.K. Seville and R. Clift, The effect of thin liquid layers on fluidisation characteristics, Powder Technology, 37 (1984) 117.

42 R.H. Ion, H.M. Pollock and C. Roques-Carmes, Micron-scale indentation of amorphous and drawn PET surfaces, J. Mat. Sci., 25 (1990) 1444.

43 J.A.S. Cleaver, Velocity Distributions in Conical Hoppers, Ph.D. Thesis, University of Cambridge, Cambridge, (1991).

44 P.A. Shamlou, Handling of Bulk Solids, theory and practice, Butterworths, London, (1988).

45 L. Svarovsky, Powder Testing Guide, Elsevier Applied Science, London, (1987).

46 R.M. Nedderman, The theoretical prediction of stress distributions in hoppers, Trans. IChemE., 60 (1982) 259.

47 J.K. Walters, A theoretical analysis of stresses in axially-symmetric hoppers and bunkers, Chem. Eng. Sci., 28 (1973) 779.

48 D.M. Walker, An approximate theory for pressures and arching in hoppers, Chem. Eng. Sci., 21 (1966) 975.

49 A.W. Jenike, Steady gravity flow of frictional-cohesive solids in converging channels, ASME, J. Appl. Mech., 31, E (1964) 5.

50 G. Enstad, On the theory of arching in mass flow hoppers, Chem. Eng. Sci., 30 (1975) 1273.

51 C.M. Martin, M. Ghadiri, U. Tüzün and B. Formisani, Effect of the electrical clamping forces on the mechanics of particulate solids, Powder Technology, 65 (1991) 37.

52 C.M. Martin, M. Ghadiri and U. Tüzün, An electromechanical valve for flow control of granular materials, 2nd World Congress Particle Technology, Kyoto, 19-22 Sept. 1990.

53 T.K. Lo, Computer flow control of electromechanical valve for solids, Final Year Undergraduate Research Report, Dept. of Chem. and Proc. Eng., University of Surrey, Guildford, (1988). 
F.M. Clark, Insulating Materials for Design and Engineering Practice, John Wiley and Sons, New York, (1962).

56 P.W. Dietz, Fields and forces of a macroscopic sphere above a ground plane in a sinusoidally varying electric field, J. Appl. Phys., 48, 3 (1977) 1036.

57 P.A. Engel, Impact Wear of Materials, Elsevier Science, Amsterdam, (1984).

58 K.L. Johnson, Contact Mechanics, Cambridge University Press, Cambridge, (1985).

59 C. Thornton, Computer-simulated experiments on particulate materials, in Tribology in Particulate Technology, B.J. Briscoe and M.J. Adams eds., Adam Hilger, Bristol, (1987) 292.

60 O.R. Walton and L.B. Braun, Granular flow: Particle dynamics simulations of steady flow, Solids Transport, US-DOE Pittsburgh Energy Tech. Centre, Sept. 17-18 1987.

61 K. Kendall, Relevance of contact mechanics to powders - Elasticity, friction and agglomerate strength, in Tribology in Particulate Technology, B.J. Briscoe and M.J. Adams eds., Adam Hilger, Bristol, (1987) 110.

62 U. Tüzün, M.J. Adams and B.J. Briscoe, The theoretical prediction of the effect of particle properties on the wall friction angles measured for smooth silo walls, Chem. Eng. Sci., 43 (1988) 1083.

63 T.B. Jones, G.L. Whittaker and T.J. Sulenski, Mechanics of magnetic powders, Powder Technology, 49 (1987) 149.

64 R.L. Handy, The arch in soil arching, J. Geotechn. Engng., 111, 3 (1985) 302 .

65 H. Rumpf, The strength of granules and agglomerates, in Agglomeration, ed. W.A. Knepper, Wiley-Interscience, New York, (1962) 379.

M. Abdel-Ghani, J.G. Petrie, J.P.K. Seville, R. Clift and M.J. Adams, Mechanical properties of cohesive particulate solids, Powder Technology, 65 (1991) 113.

67 T. Travers, M. Ammi, D. Bideau, A. Gervois, J.C. Messager and J.P. Troadec, Mechanical size effects in $2 \mathrm{~d}$ granular media, $J$. Phys. France, 49 (1988) 939.

68 T.B. Jones and R.D. Miller, Multipolar interactions of dielectric spheres, J. Electrostatics, 22 (1989) 231.

69 J.E.P. Morgan, Electromechanical valve for solids, Internal Report, Dept of Chem. and Proc. Eng., University of Surrey, Guildford, (1986).

70 W.A. Beverloo, H.A. Leniger and J. van de Velde, The flow of granular solids through orifices, Chem. Eng. Sci., 15 (1961) 260. 
R.M. Nedderman, U. Tüzün, S.B. Savage and G.T. Houlsby, The flow of granular materials-I, Chem. Eng. Sci., 37 (1982) 1597.

K.L. Johnson, K. Kendall and A.D. Roberts, Surface energy and the contact of elastic solids, Proc. Roy. Soc. Lond. A., 324 (1971) 301.

P.A. Arteaga, M.Ghadiri, N.S. Lawson and H.M. Pollock, Use of nanoindentation to assess potential attrition of particulate solids, Tribology International, 26, 1 (1993) 305.

74 C.M. Martin, P.A. Arteaga, M. Ghadiri and U. Tüzün, Characterisation of the single contact electrical clamping force, 1st Int. Part. Tech. Forum, Denver, 17-19 Aug 1994.

75 P.H. Shipway and I.M. Hutchings, Fracture of brittle spheres under compression and impact loading, Philos. Mag., A67 (1993) 1389.

76 G.W.C. Kaye and T.H. Laby, Tables of Physical and Chemical Constants, 13th ed., Longmans, London, (1966).

77 H.A. Mizes, Adhesion of small particles in electric fields, $J$. Adhesion Sci. Technol., 8, 8 (1994) 937.

78 B.R. Lawn and T.R. Wilshaw, Fracture of Brittle Solids, Cambridge University Press, Cambridge, (1975).

79 K. Kendall, The adhesion and surface energy of elastic solids, $J$. Phys. D: Appl. Phys., 4 (1971) 1186.

80 S. Tolansky, An Introduction to Interferometry, 2nd ed., Longman, London, (1973).

81 A.K. Jonscher, Time- and frequency-resolved flow of surface charge on insulators, Electrostatic Charge Migration Meeting of Institute of Physics, London, 9 March 1988.

82 B.V. Derjaguin, V.M. Muller and Yu.P. Toporov, Effect of contact deformations on the adhesion of particles, J. Coll. Int. Sci., 53, 2 (1975) 314.

83 J. Israelachvili, Y.L. Chen, H. Yoshizawa, S. Steinberg, G. Vigil and $\mathrm{Z}$. Xu, Experiments on the relation between adhesion and friction, 1st Int. Part. Tech. Forum, Denver, 17-19 Aug 1994.

S.P. Timoshenko and J.N. Goodier, Theory of Elasticity, 3rd ed., McGraw-Hill, New York, (1970). 


\section{APPENDIX 4.1}

\section{REVIEW OF ELECTRICAL CLAMPING FORCE MODELS FOR SPHERES}

The purpose of this Appendix is to review the physical and electrical concepts involved in developing the electrical clamping force models and to record these models in their final mathematical forms for reference from the main text. The detailed derivations of the electrical clamping force models are documented well in the literature $[15,16,19,20,39]$.

\section{A4.1.1 GENERAL THEORY}

The generation of electrical clamping forces between resistive particles occurs when a small current passes over the surfaces or through the volume of the particles via their points of contacts. Near the points of contact the current is constricted to flow through a small area which causes high electrical resistances. The high electrical resistances cause very high electric fields to be generated across the surfaces of the contacting particles. These electric fields in the gaps outside the contact points produce the electrical clamping forces between the particles.

The electrical clamping force models are based on the assumptions that the two particles in contact are spherical and uniform in size. The models can also be applied to the contact between a sphere and a conductive wall. In most cases it is assumed that current flow is over the surface of the particles, although Moslehi and Self [16] have also considered the volume conduction case which will be discussed below.

The general review of the theory which is given below follows that of McLean [15] because, conceptually, the mathematical development of McLean gives the most direct explanation of the real physical and electrical processes being modelled. However, McLean does not rigorously solve the equations to give exact formulations of the electrical clamping force models. Dietz [19] and Moslehi and Self [16] developed their models based on the same theoretical principles but used different numerical methods of solution. The relevant details and the final formulations of their models will be reviewed further below.

For surface conduction over two contacting spherical particles, the 
effective surface resistance from the angle of contact, $\theta_{0}$, to another point on the surface given by angle $\theta$ can be expressed from the general relationship between resistance and resistivity. From the more typical application of a homogeneous material between two parallel plate electrodes, the relationship between volume resistance and volume resistivity holds that

$$
R_{b}=\rho_{b} \frac{L}{A}
$$

where $R_{b}$ is the bulk volume resistance between the plates, $\rho_{b}$ is the bulk volume resistivity of the material between the plates, $L$ is the length of the current path between the plates, and $\mathrm{A}$ is the cross-sectional area of the plates over which the current flows. The relationship between resistance and resistivity given by equation (A4.1) can be applied to the case of surface conduction in the spherical system shown in Fig. A4.1 where current is flowing over the surface of the sphere with radius $R$ from one contact point to the next. For the case of Fig. A4.1, an effective length $L^{\prime}$ for the current path can be defined such that

$$
\Delta \mathrm{L}^{\prime}=\mathrm{R} \Delta \theta
$$

and an effective 'area' $A^{\prime}$ over which the current is constricted can be defined such that

$$
A^{\prime}=2 \pi R \sin \theta
$$

where $A^{\prime}$ in this case is really the circular perimeter around the sphere through which the current flows.

The surface resistance of one sphere from the angle of contact, $\theta_{0}$, through to an angle on the surface given by $\theta$ is found by integrating equations (A4.2) and (A4.3) according to the relationship given by equation (A4.1) such that

$$
\begin{aligned}
\mathrm{R}_{\theta} & =\frac{\rho_{\mathrm{s}}}{2 \pi} \int_{\theta_{0}}^{\theta} \frac{\mathrm{d} \theta}{\sin \theta} \\
& =\frac{\rho_{\mathrm{s}}}{2 \pi} \ln \left(\frac{\tan \frac{\theta}{2}}{\tan \frac{\theta_{0}}{2}}\right)
\end{aligned}
$$




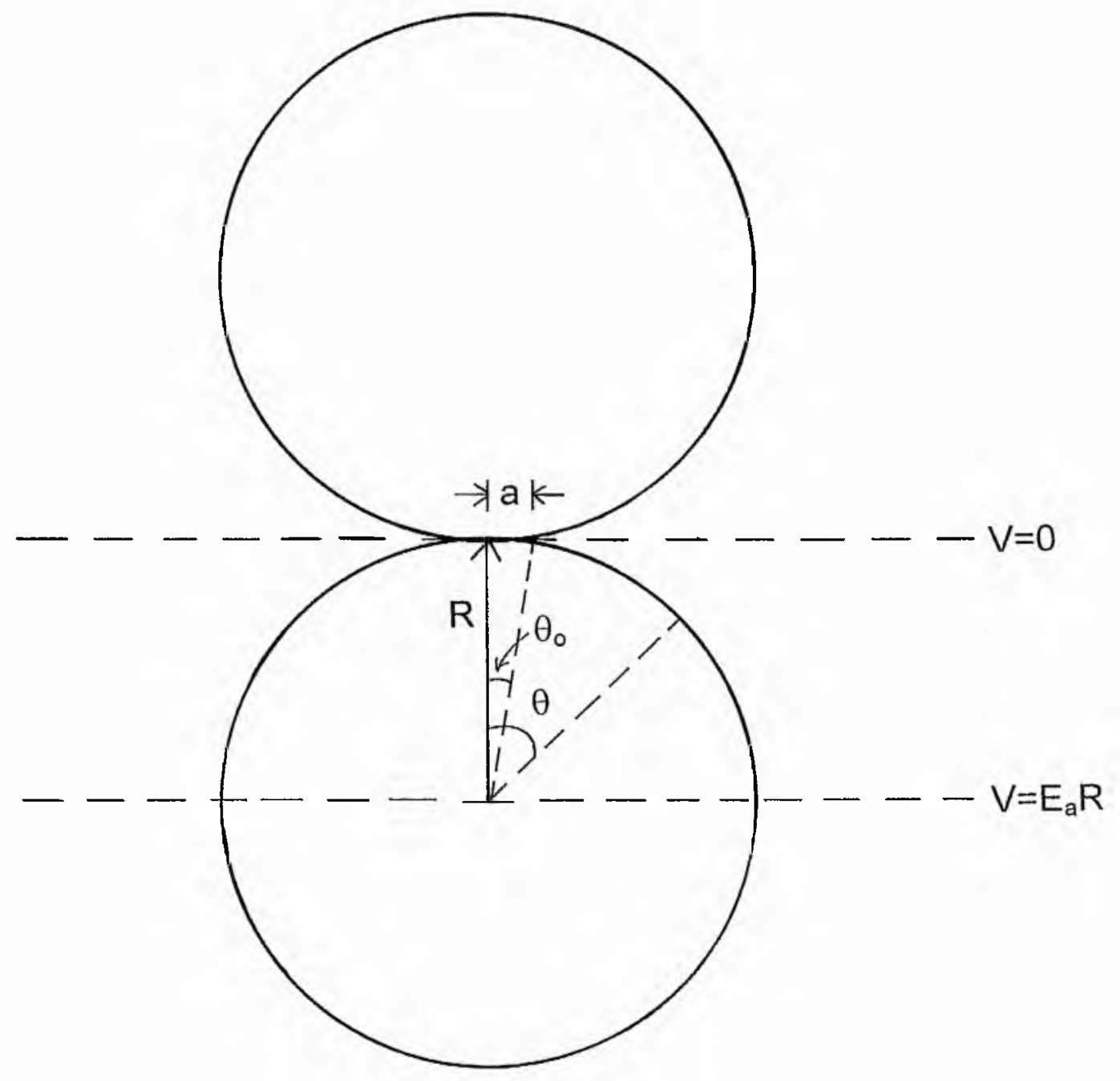

Fig. A4.1 Contact between two spheres with applied electric field $E_{a}$. 
where $\rho_{s}$ is the surface resistivity of the sphere.

The voltage potential at any angle $\theta$ on the surface of the sphere can now be found by equating the electric current at angle $\theta$ to the current at another point on the sphere because current is conserved. Therefore,

$$
I_{\theta}=I_{\pi / 2}
$$

where $I_{\theta}$ is the current at any angle $\theta$ and $I_{\pi / 2}$ is the current at $\theta=\pi / 2$.

From Ohm's Law, the current can be expressed simply as

$$
I=\frac{V_{\pi / 2}}{R_{s, \pi / 2}}=\frac{V_{\theta}}{R_{s, \theta}}
$$

where $R_{s}$ is the resistance of the sphere at the specified angle.

Rearranging equation (A4.6) gives

$$
V(\theta)=\frac{R_{s, \theta} V_{\pi / 2}}{R_{s, \pi / 2}}
$$

If the average electric field applied across the particulate assembly in the direction normal to the contact is $E_{a}$, and the voltage at the contact is arbitrarily set to be zero, then the voltage potential at $\theta=\pi / 2$ is given by

$$
\mathrm{V}_{\pi / 2}=\mathrm{R} \cdot \mathrm{E}_{\mathrm{a}}
$$

By substituting equations (A4.4) and (A4.8) into equation (A4.7), the voltage potential at any angle $\theta$ on the surface of the sphere relative to the contact point is given by

$$
\mathrm{V}(\theta)=R \mathrm{E}_{\mathrm{a}} \frac{\ln \left(\frac{\tan \frac{\theta}{2}}{\tan \frac{\theta}{2} \mathrm{o}}\right)}{\ln \left(\frac{1}{\tan \frac{\theta}{2} \mathrm{o}}\right)}
$$


The electric field in the gap between the surfaces of the two contacting spheres, in the direction normal to the contact as defined by the $z$ axis, at angle $\theta$ is given by

$$
E_{z}(\theta)=\frac{V(\theta)}{Z(\theta)}
$$

where $Z(\theta)$ is the distance from the surface of the sphere at $\theta$ to the plane of the contact point and is given by

$$
Z(\theta)=R\left(\cos \theta_{0}-\cos \theta\right)
$$

By substituting equations (A4.11) and (A4.9) into equation (A4.10), the electric field in the gap between the sphere surfaces is given by

$$
E_{z}(\theta)=\frac{E_{a} \ln \left(\frac{\tan \frac{\theta}{2}}{\tan \frac{\theta}{2} o}\right)}{\left(\cos \theta_{0}-\cos \theta\right) \ln \left(\frac{1}{\tan \frac{\theta}{2} o}\right)}
$$

The total force of attraction between the surfaces of the two particles is found by integrating the incremental force over the area of the plane on which the force is acting, ie. the contact plane, between $r_{s}=a$ and $r_{s}=$ $R$, where $r_{s}$ is the radial distance from the centre of the contact in the direction parallel to the contact. The result of this integration is relatively insensitive to the actual value of the upper boundary $[15,19]$. Therefore, the electrical clamping force between two particles is given by

$$
f_{e}=\int_{a}^{R} \frac{\varepsilon_{f}}{2} E_{z}^{2} 2 \pi r_{s} d r_{s}
$$

If the contact cap size was fixed by the mechanical loading on the contact and was not affected by the electrical clamping force, then the value of the contact cap radius, a, in equation (A4.13) and the value of the contact angle, $\theta_{0}$, in equation (A4.12) would be fixed. Based on this assumption, equation (A4.13) is solved to give the Fixed Cap model of the electrical clamping force. The formulations of the Fixed Cap models of Dietz [19] and Moslehi and Self [16] are discussed below. In the Fixed Cap case, the 
electrical clamping force exhibits a squared dependence on the average applied electric field, $\mathrm{E}_{\mathrm{a}}$.

However, due to the elastic behaviour of solids the contact cap size will increase upon application of the electrical clamping force. McLean [15], Dietz [19], and Moshlehi and Self [16] all assume that the contact cap size is related to the electrical clamping force by Hertz theory [57,58] such that

$$
a=\left(\frac{3 \pi f}{8 R_{e f f} E_{\text {eff }}}\right)^{1 / 3}
$$

where $\quad R_{\text {eff }}=\frac{1}{2}\left(\frac{1}{R_{1}}+\frac{1}{R_{2}}\right)$

$$
E_{e f f}=\left(\frac{1-v_{r 1}^{2}}{\pi E_{m 1}}+\frac{1-v_{r 2}^{2}}{\pi E_{m 2}}\right)^{-1}
$$

In equation (A4.14), $R$ is the particle radius, $E_{m}$ is the Elastic Modulus and $v_{r}$ is the Poisson's ratio where the subscripts 1 and 2 reference the two contacting bodies. The Elastic Cap model of the electrical clamping force is obtained by solving equations (A4.13) and (A4.14) simultaneously. The formulations of Dietz [19] and Moslehi and Self [16] are given below. In the Elastic Cap case, the order of dependence of the electrical clamping force on the applied electric field, $E_{a}$, is reduced from that of the Fixed Cap model to 1.2 to 1.5 .

In the above analysis of the electrical clamping force, it was assumed that the electric field given by equation (A4.12) which exists in the gap between the particles' surfaces is unlimited. Depending on the average applied field $E_{a}$ and the contact cap size, it is possible that sufficiently high electric fields could be produced in the contact gap to cause electrical breakdown of the interstitial gas. In this case, the electric charge is transferred from one particle surface to the other through the gas, bypassing the contact point between the surfaces.

This behaviour can be modelled by assuming that the electric field in the gap, $E_{z}$, has a maximum allowable value, $E_{\text {max' }}$ between a critical angle, $\theta_{c}$. and the contact angle, $\theta_{0}$. The electric field distribution in the gap is then related to $\mathrm{E}_{\mathrm{a}}$ such that 


$$
E_{\max } Z\left(\theta_{c}\right)+\int_{\theta_{c}}^{\pi / 2} E_{z}(\theta) R d \theta=E_{a} R
$$

If the value of $E_{\max }$ is known, equations (A4.13) and (A4.15) for the electrical clamping force can be solved numerically to give the Limited Field model. However, it is doubtful whether $E_{\max }$ can simply be assumed to be the breakdown strength of air, $3 \times 10^{6} \mathrm{~V} \mathrm{~m}^{-1}$, because the mechanisms of electrical breakdown are not well defined for the very small gap sizes of relevance to this work. Where the gap is of the order of the mean free path of the gas, much higher field strengths are required for breakdown [16] and the exact breakdown limit becomes difficult to define [19]. Only Dietz [19] has solved for a formulation of the Limited Field model, in which he assumes that $\mathrm{E}_{\max }$ is equal to $3 \times 10^{6} \mathrm{~V} \mathrm{~m}^{-1}$. His model is given below. In the Limited Field model, the order of dependence of the electrical clamping force on the applied field is reduced to being approximately linear.

In general, all the models for the electrical clamping force can be expressed in the form

$$
\mathrm{f}_{\mathrm{e}}=\alpha \mathrm{E}_{\mathrm{a}}^{\gamma}
$$

where $\alpha$ is a function of the physical and electrical properties of the particles and the interstitial fluid, and $\gamma$ is a power law constant.

\section{A4.1.2 MODELS OF DIETZ [19]}

By following a similar theoretical approach to that described above, Dietz [19] obtained the following formulations from numerical solutions to the equations for the Fixed Cap, Elastic Cap, and Limited Field models:

\section{Fixed Cap}

$$
\mathrm{f}_{\mathrm{e}}=\frac{(0.4) 4 \pi \varepsilon_{\mathrm{f}} \mathrm{R}^{2}\left(\frac{\mathrm{R}}{\mathrm{a}}\right)^{\overline{2}} \mathrm{E}_{\mathrm{a}}{ }^{2}}{\ln ^{2}\left(\frac{\mathrm{a}}{2 \mathrm{R}}\right)}
$$

\section{Elastic Cap}

$$
\mathrm{f}_{\mathrm{e}}=2.0 \mathrm{R}^{2} \varepsilon_{\mathrm{f}}^{0.65} \mathrm{E}_{\mathrm{m}}^{0.35} \mathrm{E}_{\mathrm{a}}^{1.3}
$$


where $\mathrm{E}_{\mathrm{m}}$ is the Elastic Modulus of the particles.

\section{Limited Field}

$f_{e}=(0.18) 4 \pi \varepsilon_{f} R^{2} E_{\max }^{0.946} E_{a}^{1.054}$

$$
\log \frac{E_{a}}{E_{\max }} \geq-2
$$

$f_{e}=(0.415) 4 \pi \varepsilon_{f} R^{2} E_{\max }^{0.8} E_{a}^{1.2}$

$$
\log \frac{E_{a}}{E_{\max }} \leq-3
$$

One minor difference in Dietz' mathematical development of the Elastic Cap model from the general theory is his use of a simplified formulation for the Hertzian contact cap radius as compared to equation (A4.14). Dietz uses the following relationship for contact radius as taken from Timoshenko and Goodier [84]:

$$
a=0.87\left(\frac{R f_{e}}{E_{m}}\right)^{1 / 3}
$$

where $f_{e}$ is the load on the contact, in this case given by the electrical clamping force. There is negligible difference between equation (A4.21) and equation (A4.14), as can be seen by rewriting equation (A4.14) for the contact between two particles of the same diameter and same Elastic modulus, such that

$$
a=0.91\left(\frac{R f_{e}\left(1-v_{r}^{2}\right)}{E_{m}}\right)^{1 / 3}
$$

where $v_{r}$ is the Poisson's ratio. For the materials of interest in this work, ie. glass and sand, $v_{r}$ is approximately 0.2 so $\left(1-v_{r}{ }^{2}\right)$ is approximately 1. Therefore, there will be negligible difference in the contact cap sizes calculated from equations (A4.21) and (A4.22).

As the Elastic Cap model is obtained by solving the equations for the Fixed Cap model and the contact cap size simultaneously, the Fixed Cap model and the Elastic Cap model should give consistent results. This is not the case with the Fixed Cap model, equation (A4.17), and the Elastic Cap model, equation (A4.18), of Dietz as is shown by the following example, where $R=425 \times 10^{-6} \mathrm{~m}, E_{m}=8 \times 10^{10} \mathrm{~N} \mathrm{~m}^{-2}$, and $\varepsilon_{\mathrm{f}}=8.86 \times 10^{-12} \mathrm{~F} \mathrm{~m}^{-1}$. 
From the Elastic Cap model equation (A4.18),

$$
\text { for } \begin{aligned}
E_{a} & =2.19 \times 10^{5} \mathrm{~V} \mathrm{~m}^{-1}, \\
f_{e} & =1.35 \times 10^{-3} \mathrm{~N} .
\end{aligned}
$$

From equation (A4.21),

$$
a=1.68 \times 10^{-6} \mathrm{~m} \text {. }
$$

From the Fixed Cap model equation (A4.17),

$$
\text { for } \begin{aligned}
E_{a} & =2.19 \times 10^{5} \mathrm{~V} \mathrm{~m}^{-1} \text { and } \mathrm{a}=1.68 \times 10^{-6} \mathrm{~m} \\
\mathrm{f}_{\mathrm{e}} & =0.64 \times 10^{-3} \mathrm{~N} .
\end{aligned}
$$

The above example shows that the electrical clamping forces calculated from the Fixed Cap and Elastic Cap models of Dietz are not the same, which indicates an inconsistency in the numerical method of solution of the equations.

\section{A4.1.3 MODELS OF MOSLEHI AND SELF [16]}

The analysis of Moslehi and Self [16] considers both cases of surface and volume conduction. They follow similar reasoning in their theoretical development as reviewed above, although their mathematical approach is considerably different from that of McLean [15] and Dietz [19]. The details of the mathematics will not be repeated here, however a brief discussion comparing the relative magnitudes of the surface and volume force predictions is presented and the models are compared to Dietz'.

\section{Fixed Cap Model}

Moslehi and Self developed the general equation for surface and volume conduction for the Fixed Cap electrical clamping force such that

$$
f_{e}=\pi R^{2} \varepsilon_{f} E_{1 i m}^{2} \theta_{0}^{2} A
$$

where $E_{\text {lim }}$ is the limiting value of the electric field in the gap which occurs as $\theta$ is taken to its limiting value of $\theta_{0}$, and $A$ is a constant with no physical meaning. The values of $E_{\text {lim }}$ and $A$ differ for the cases of surface and volume conduction such that 
(i) for surface conduction

$$
\begin{gathered}
E_{\lim }=\frac{1}{\theta_{0}^{2} \ln \left(\frac{2}{\theta_{0}}\right) E_{a}} \\
A_{s}=2.14
\end{gathered}
$$

(ii) for volume conduction

$$
\begin{aligned}
\mathrm{E}_{\mathrm{lim}} & =\frac{1}{\theta_{0}^{2}} \mathrm{E}_{\mathrm{a}} \\
\mathrm{A}_{v} & =1.28
\end{aligned}
$$

By combining equations (A4.23-27), the ratio of the surface conduction force to the volume conduction force is such that

$$
\frac{\left(f_{e}\right)_{\text {surface }}}{\left(f_{e}\right)_{\text {volume }}}=\frac{1.67}{\ln ^{2}\left(\frac{2}{\theta_{0}}\right)}
$$

For values of $\theta_{0}$ ranging from $0.1^{\circ}-1.0^{\circ}\left(1.75 \times 10^{-3}-1.75 \times 10^{-2} \mathrm{rad}\right)$,

$$
f_{\text {e surface }}=(0.033-0.076) f_{\text {e volume }}
$$

Therefore for the Fixed Cap model, the electrical clamping forces produced by surface conduction are predicted to be considerably smaller than the volume conduction forces. As discussed in Chapter 4, surface conduction is assumed to dominate over volume conduction for the solids, and temperature and humidity conditions relevant for this work.

The Fixed Cap model of Moslehi and Self for surface conduction can be written in a form similar to Dietz' equation (A4.17) by using the above equations and the relationship

$$
\theta_{0}=\left(\frac{\mathrm{a}}{\mathrm{R}}\right)
$$

By combining equations (A4.23-25) and (A4.30), the Fixed Cap model of Moslehi and Self can be written as 


$$
f_{e}=\frac{2.14 \pi \varepsilon_{f} R^{2}\left(\frac{R}{a}\right)^{2} E_{a}^{2}}{\ln ^{2}\left(\frac{a}{2 R}\right)}
$$

Comparing equations (A4.17) and (A4.31) shows that the Fixed Cap models of Dietz and Moslehi and Self are very similar. The force predicted by Moslehi and Self's model will be about $34 \%$ larger than the force predicted by Dietz' model.

\section{Elastic Cap Model}

Moslehi and Self develop their Elastic Cap model similarly to Dietz [19] and McLean [15], by simultaneously solving equation (A4.23) for the Fixed Cap force and equation (A4.14) for the Hertzian contact radius. Moslehi and Self consider only the contact between two spheres of the same diameter and elastic properties when solving for the Elastic Cap model. Their Elastic Cap model equation for surface conduction has been modified here to apply to both cases of the contact between two spheres of the same diameter and for the contact between a sphere and a conducting wall. The equations are applicable to the latter case by setting $R_{2}$ equal to infinity in equation (A4.14) for the calculation of $E_{\text {eff }}$ and $R_{\text {eff }}$.

(i) Surface conduction

$$
\begin{gathered}
f_{e}=\pi R_{1}^{2} \varepsilon_{f} A_{s} D_{s}^{-2 / 5}\left(\frac{E_{a}}{K_{s}}\right)^{6 / 5} \\
D_{s}=\frac{3 \pi^{2} \varepsilon_{f} A_{s}}{8 R_{1} R_{\text {eff }} E_{\text {eff }}} \\
K_{s}=\left(\frac{1}{5}\right) \ln \left\{32 D_{s}^{-1}\left(\frac{E_{a}}{K_{s}}\right)^{-2}\right\}
\end{gathered}
$$

where $A_{s}=2.14$ as for the Fixed Cap model.

Moslehi and Self's model for the Elastic Cap force for volume conduction has not been modified to extend to the contact between a sphere and wall because the case of volume conduction was not considered relevant for this work. Therefore, the magnitudes of the Elastic Cap force for surface and volume conduction are compared below only for the case of contact between 
two spheres of the same diameter. For this analysis, it is convenient to use the simplified expression for $\mathrm{D}_{\mathrm{s}}$ for the contact between two spheres given by

$$
D_{s}=\frac{3 \pi \varepsilon_{f} A_{s}\left(1-v_{r}^{2}\right)}{4 E_{m}}
$$

(ii) Volume conduction

$$
\begin{aligned}
& f_{e}=\pi R^{2} \varepsilon_{f} A_{v} D_{v}^{-2 / 5} E_{a}^{6 / 5} \\
& D_{v}=\frac{3 \pi \varepsilon_{f} A_{v}\left(1-v_{r}^{2}\right)}{4 E_{m}}
\end{aligned}
$$

where $A_{v}=1.28$ as for the Fixed Cap model.

By combining equations (A4.32-37), the ratio of the surface conduction force to the volume conduction force is such that

$$
\frac{\left(f_{e}\right) \text { surface }}{\left(f_{e}\right)_{\text {volume }}}=1.36\left(\frac{1}{\mathrm{~K}_{\mathrm{s}}}\right)^{6 / 5}
$$

Using the values of $v_{r}$ and $\mathrm{E}_{\mathrm{m}}$ for glass as 0.25 and $6.2 \times 10^{10} \mathrm{~N} \mathrm{~m}^{-2}$ respectively in equation (A4.35), the value of $K_{s}$ from equation (A4.34) ranges from 9.5 to 5.6 over an applied field range of $10^{2}$ to $10^{6} \mathrm{~V} \mathrm{~m}^{-1}$. Using an average value for $\mathrm{K}_{\mathrm{s}}$ of 8 in equation (A4.38) gives a value of 0.1 for the ratio of surface conduction force to volume conduction force. Therefore, just as for the Fixed Cap model, the Elastic Cap model predicts that surface conduction forces will be considerably smaller than volume conduction forces. The remaining analyses will consider only the surface conduction Elastic Cap model as volume conduction is not considered relevant for this work.

In the form of equations (A4.32-35), it is difficult to compare the Elastic Cap model of Moslehi and Self to that of Dietz in equation (A4.18). By making some simplications to Moslehi and Self's model, an approximate comparison can be made. If $\left(1-v_{r}^{2}\right)$ in equation (A4.35) is assumed to be approximately 1 , and $\mathrm{K}_{\mathrm{s}}$ is taken as 8 , then equations (A4.32-35) for the Elastic Cap model simplify to give 


$$
\mathrm{f}_{\mathrm{e}}=0.3 \mathrm{R}^{2} \varepsilon_{\mathrm{f}}^{0.6} \mathrm{E}_{\mathrm{m}}^{0.4} \mathrm{E}_{\mathrm{a}}^{1.2}
$$

Comparing equations (A4.39) and (A4.18) shows that the forms of the Elastic Cap models for Moslehi and Self and Dietz are very similar.

It is of interest to consider whether the Elastic Cap and Fixed Cap models of Moslehi and Self are self-consistent whereas the models of Dietz are not. The consistency of Moslehi and Self's models is checked in the following example calculation where $\mathrm{R}=425 \times 10^{-6} \mathrm{~m}, \mathrm{E}_{\mathrm{m}}=8 \times 10^{10} \mathrm{~N} \mathrm{~m}^{-2}$, $v_{r}=0.17$ and $\varepsilon_{f}=8.86 \times 10^{-12} \mathrm{~F} \mathrm{~m}^{-1}$.

From the Elastic Cap model equations (A4.32-35),

$$
\text { for } \begin{aligned}
E_{a} & =2.84 \times 10^{5} \mathrm{~V} \mathrm{~m}^{-1}, \\
f_{e} & =1.35 \times 10^{-3} \mathrm{~N} .
\end{aligned}
$$

From equation (A4.14),

$$
a=1.74 \times 10^{-6} \mathrm{~m} \text {. }
$$

From the Fixed Cap model equation (A4.31)

$$
\text { for } \begin{aligned}
E_{a} & =2.84 \times 10^{5} \mathrm{~V} \mathrm{~m}^{-1} \text { and } a=1.74 \times 10^{-6} \mathrm{~m}, \\
f_{e} & =1.35 \times 10^{-3} \mathrm{~N} .
\end{aligned}
$$

Therefore, the Fixed Cap and Elastic Cap models of Moslehi and Self are self-consistent and were chosen over those of Dietz for the macroscopic and microscopic analyses of the electrical clamping forces in this work. 


\section{APPENDIX 4.2}

\section{ARCH MODELLING}

\section{A4.2.1 DEFINITION OF ANGLE $\beta$ IN ARCH MODEL}

As defined by Enstad [50], the angle $\beta$ is the angle between the major principal stress and the normal to the wall (or between the major principal stress plane and the wall). The angle $\beta$ is used to define the stresses in the arched differential element shown in Fig. A4.2. The value of $\beta$ can be determined from Fig. A4.3 which shows a Mohr circle representation of the stresses in the material. The stresses and angles for passive and active stress states are indicated using subscripts $p$ and a respectively.

For the passive case, $\triangle A O C$ is considered. By equating the sum of the internal angles in $\triangle A O C$ to $\pi$, an expression for $\beta_{p}$ is obtained as

$$
\beta_{p}=\frac{1}{2}\left(\phi+\nu_{p}\right)
$$

Applying the sine law to $\triangle \mathrm{AOC}$ gives

$$
\sin v_{\mathrm{p}}=\frac{\sigma}{\mathrm{R}_{\mathrm{m}}} \sin \phi
$$

From $\triangle \mathrm{DOC}$,

$$
\frac{R_{m}}{\sigma}=\sin \delta
$$

Combining equations (A4.41) and (A4.42) gives an expression for $v_{p}$ as

$$
\sin \nu_{p}=\frac{\sin \phi}{\sin \delta}, \quad \text { where } 0 \leq \nu_{p} \leq \frac{\pi}{2}
$$

By applying the same geometrical rules to $\triangle B O C$ for the active stress state, the value of $\beta_{a}$ can be obtained from the following expressions:

$$
\begin{gathered}
\beta_{a}=\frac{1}{2}\left(\phi+v_{a}\right) \\
\sin \nu_{a}=\frac{\sin \phi}{\sin \delta}, \quad \text { where } \frac{\pi}{2} \leq \nu_{a} \leq \pi
\end{gathered}
$$

For the fully rough wall case, the wall yield locus touches the Mohr stress circle at only one point. Therefore, for this case there is only one 


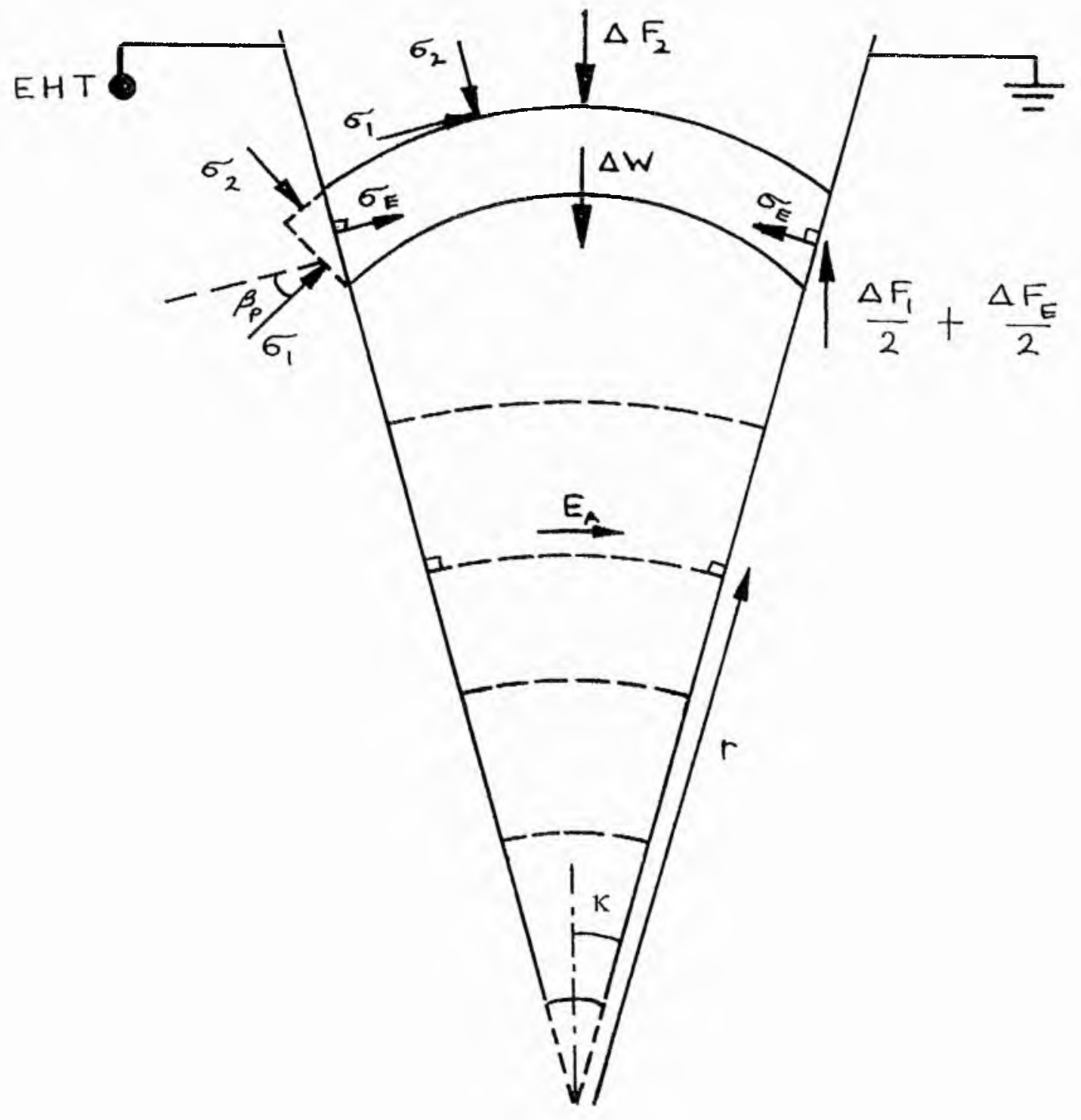

Fig. A4.2 Representation of mechanical and electrical stresses acting on arched element in wedge-shaped hopper. 


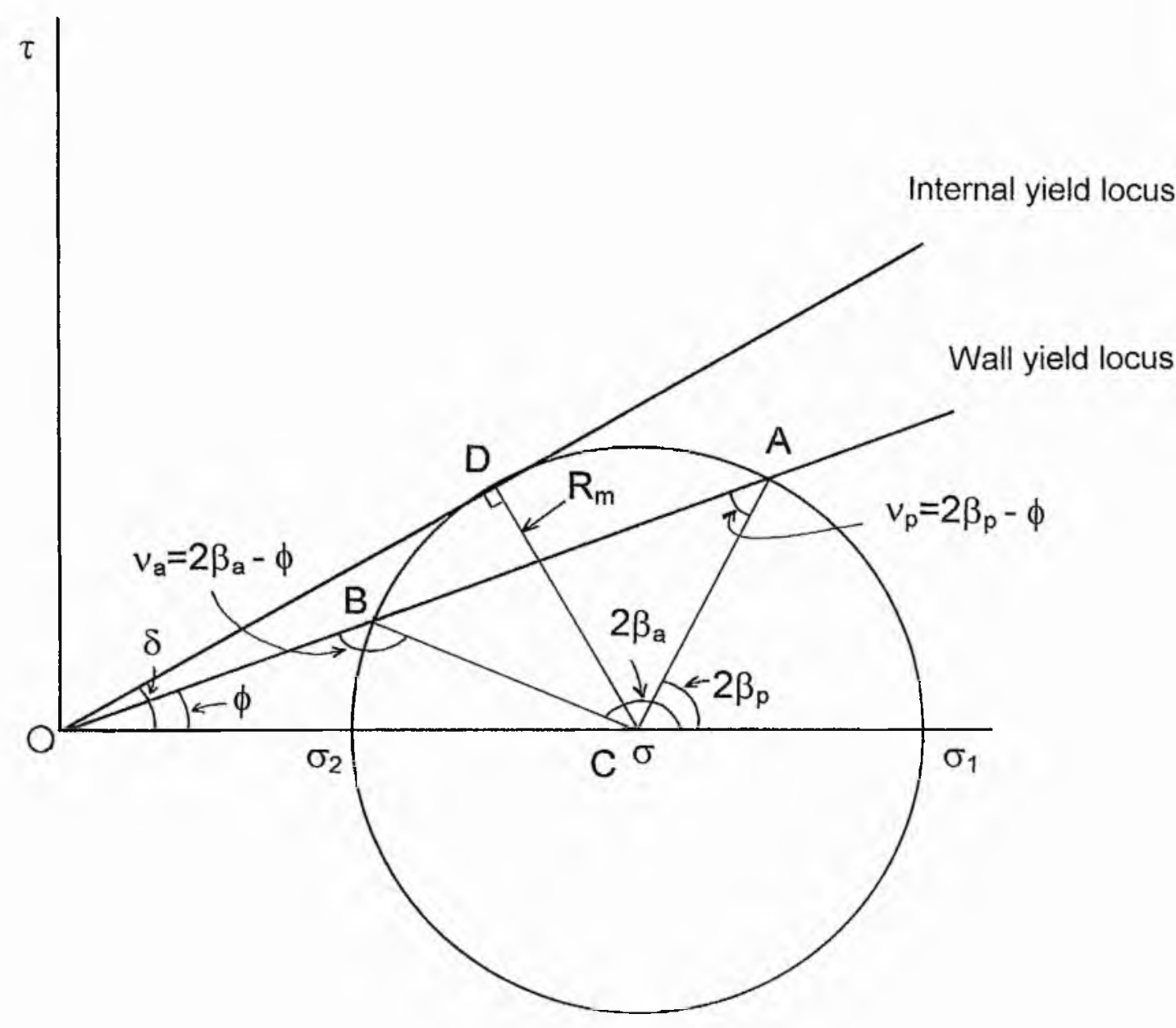

Fig. A4.3 Definition of angles used in External Stress Arch model from yield loci and Mohr's stress circle for non-cohesive material. 
stress state possible. Enstad has arbitrarily assigned the active stress state to this case where $\nu=\pi / 2$.

\section{A4.2.2 EXTERNAL STRESS ARCH MODEL FOR PASSIVE STRESS STATE}

From Fig. A4.2, there are three mechanical forces acting on the arched differential element. These forces are the weight of the layer, $\Delta W$, the net downward force from the material on either side of the layer, $\Delta \mathrm{F}_{2}$, and the lift force from the walls, $\Delta F_{1}$. To obtain expressions for these forces, the stresses on the arched element are defined by Enstad [50] such that the major principal stress, $\sigma_{1}$, is tangent to the surface of the arch and constant over the arch, and the minor principal stress, $\sigma_{2}$, is normal to the arch and constant over the arch surface. The shape of the arch is defined such that the angle between $\sigma_{1}$ and the normal to the wall is $\beta_{\mathrm{p}}$, as shown in Fig. A4.2. By considering an arched element of differential height $\Delta r$, the forces on the element per unit depth are described as follows.

The weight of the layer is given by

$$
\Delta W=2 \Gamma \Gamma \Delta \Gamma\left(\frac{\left(\beta_{p}+\kappa\right) \sin \kappa}{\sin ^{2}\left(\beta_{p}+\kappa\right)}+\frac{\sin \beta_{p}}{\sin \left(\beta_{p}+\kappa\right)}\right) \sin \kappa
$$

The net downward force is given by

$$
\Delta \mathrm{F}_{2}=2 \Delta r \sin \kappa\left(\sigma_{2}+r \frac{\mathrm{d} \sigma_{2}}{\mathrm{~d} r}\right)
$$

From Fig. A4.3,

$$
\sigma_{2}=\sigma(1-\sin \delta)
$$

where $\sigma$ is the mean principal stress given by

$$
\sigma=\frac{\sigma_{1}+\sigma_{2}}{2}
$$

Substituting equation (A4.48) into equation $(\mathrm{A} 4.47)$ gives

$$
\Delta \mathrm{F}_{2}=2 \Delta \mathrm{rsin} \kappa(1-\sin \delta)\left(\sigma+\Gamma \frac{\mathrm{d} \sigma}{\mathrm{dr}}\right)
$$


The net lift from mechanical forces at the walls is given by

$$
\Delta \mathrm{F}_{1}=2 \Delta \Gamma\left(\sigma_{1} \cos \beta_{\mathrm{p}} \sin \left(\beta_{\mathrm{p}}+\kappa\right)-\sigma_{\overline{\mathrm{z}}} \sin \beta_{\mathrm{p}} \cos \left(\beta_{\mathrm{p}}+\kappa\right)\right)
$$

From Fig. A4.3,

$$
\sigma_{1}=\sigma(1+\sin \delta)
$$

Substituting equations (A4.52) and (A4.48) into equation (A4.51) gives

$$
\Delta \mathrm{F}_{1}=2 \Delta \mathrm{r} \sigma\left(\sin \kappa+\sin \delta \sin \left(2 \beta_{\mathrm{p}}+\kappa\right)\right)
$$

From equation (4.27) in Chapter 4, the lift force at the walls due to the electrical clamping forces is

$$
\Delta F_{e}=\frac{2 \alpha}{s^{2}} \Delta r \sin \kappa\left(\frac{V}{2 \kappa r}\right)^{\gamma}
$$

Neglecting acceleration, the force balance over the arched element yields

$$
\Delta \mathrm{W}+\Delta \mathrm{F}_{2}=\Delta \mathrm{F}_{1}+\Delta \mathrm{F}_{e}
$$

Substituting equations (A4.46), (A4.50), (A4.53) and (A4.54) into equation (A4.55) gives the following differential equation:

$$
r \frac{d \sigma}{d r}-X \sigma=-\Gamma Y r+C\left(\frac{V}{r}\right)^{\gamma}
$$

where

$$
\begin{aligned}
& X=\frac{\sin \delta}{1-\sin \delta}\left(1+\frac{\sin \left(2 \beta_{p}+\kappa\right)}{\sin \kappa}\right) \\
& Y=\frac{\sin \kappa\left(\beta_{p}+\kappa\right)+\sin \beta_{p} \sin \left(\beta_{p}+\kappa\right)}{(1-\sin \delta) \sin ^{2}\left(\beta_{p}+\kappa\right)} \\
& C=\frac{\alpha 2^{-\gamma}}{S^{2}(1-\sin \delta) \kappa^{\gamma}}
\end{aligned}
$$

Solving differential equation (A4.56) using the boundary condition $\sigma(r)=$ 
$\sigma(H)$ at $r=H$ results in the final expression for $\sigma(r)$ as

$\sigma(r)=\frac{\Gamma Y_{\Gamma}}{X-1}-\frac{C}{\gamma+X}\left(\frac{V}{\Gamma}\right)^{\gamma}+\left\{\sigma(H)-\frac{\Gamma Y H}{X-1}+\frac{C}{\gamma+X}\left(\frac{V}{H}\right)^{\gamma}\right\}\left(\frac{r}{H}\right)^{X}$

(A4.60)

\section{A4.2.3 EXTERNAL STRESS ARCH MODEL FOR ACTIVE STRESS STATE}

In the active stress state, Enstad [50] defines the shape of the arch such that the minor principal stress is tangent to the arch and constant in value over the arch surface, and the major principal stress is normal to the arch and constant in value over the arch surface. The angle between the major principal stress and the normal to the wall is defined as $\beta_{a}$. Since in the active case the arch follows the direction of minor principal stress, the angle between the arch and the normal to the wall is $\pi / 2-\beta_{a}$. By interchanging $\sigma_{1}$ and $\sigma_{2}$ and substituting $\beta_{\mathrm{p}}$ with $\pi / 2-\beta_{\mathrm{a}}$ in Fig. A4.2, the mechanical forces acting on the arched element are given by the following equations:

$$
\begin{gathered}
\Delta \mathrm{W}=2 \Gamma r \Delta r\left(\frac{\sin \kappa\left(\kappa+\beta_{\mathrm{a}}-\pi / 2\right)}{\cos ^{2}\left(\kappa+\beta_{\mathrm{a}}\right)}+\frac{\cos \beta_{\mathrm{a}}}{\cos \left(\kappa+\beta_{\mathrm{a}}\right)}\right) \sin \kappa \\
\Delta \mathrm{F}_{2}=2 \Delta r \sin \kappa(1+\sin \delta)\left(\sigma+\mathrm{r} \frac{\mathrm{d} \sigma}{\mathrm{d} r}\right) \\
\Delta \mathrm{F}_{1}=2 \Delta \mathrm{r \sigma}\left(\sin \kappa+\sin \delta \sin \left(2 \beta_{\mathrm{a}}+\kappa\right)\right)
\end{gathered}
$$

The lift force at the walls due to the electrical clamping forces is given by equation (A4.54). Solving the force balance given in equation (A4.55) results in the same differential equation given in equation (A4.56), where the expressions for $\mathrm{X}, \mathrm{Y}$, and $\mathrm{C}$ are now given by

$$
\begin{gathered}
X=\frac{\sin \delta}{1+\sin \ddot{0}}\left(\frac{\sin \left(2 \beta_{a}+\kappa\right)}{\sin \kappa}-1\right) \\
Y=\frac{\left(\kappa+\beta_{a}-\pi / 2\right) \sin \kappa+\cos \beta_{a} \cos \left(\beta_{a}+\kappa\right)}{(1+\sin \delta) \cos ^{2}\left(\beta_{a}+\kappa\right)}
\end{gathered}
$$




$$
C=\frac{\alpha 2^{-\gamma}}{s^{2}(1+\sin \delta) k^{\gamma}}
$$

Therefore, for the active stress case the final expression for $\sigma(r)$ is the same as that given above in equation (A4.60) with the definitions of $X, Y$, and $\mathrm{C}$ given by equations (A4.64-A4.66).

\section{A4.2.4 ELECTRICAL COHESION ARCH MODEL}

In this analysis the electrical clamping forces are no longer modelled as producing an external lifting force on the arched layer at the walls. Instead the electrical clamping forces are considered to produce interparticle cohesion in the material so that the yield locus changes. Therefore, in this case there are only three forces acting on the layer. These forces are the weight of the layer, $\Delta W$, the net downward force from the material on either side of the layer, $\Delta F_{2}$, and the lift force from the walls, $\Delta F_{1}$. These forces are given by equations (A4.46), (A4.47), and (A4.51), respectively, from the passive external stress arch model with one change: the angle $\beta_{p}$ is replaced by the angle $\omega$ (referred to as $\alpha$ in Enstad's paper [50]). As explained fully in Chapter 4, a cohesive material which forms an arch cannot be assumed to be in a fully plastic stress state. Therefore, the wall angle of friction may not be fully developed and the construction given in Fig. A4.3 cannot be used to determine the angle which the arch makes with the wall normal. For cohesive arching, Enstad [50] represents the angle formed between the arch and the wall normal by $\omega$, and considers that the formation of arches is more or less random. In his analysis, he then considers the limiting case given by the value of $\omega$ which will give the maximum lift $\Delta \mathrm{F}_{1}$. Since the maximum lift will occur in the passive stress state, this analysis is limited to the passive stress state. On physical grounds, he takes this value of $\omega$ to be limited by the wall friction angle $\phi$.

Taking equations (A4.46), (A4.47), and (A4.51) and replacing $\beta_{p}$ with $\omega$ gives the expressions for the forces on the layer in cohesive arching. These expressions are given by the following:

$$
\Delta W=2 \Gamma r \Delta r\left(\frac{(\omega+\kappa) \sin \kappa}{\sin ^{2}(\omega+\kappa)}+\frac{\sin \omega}{\sin (\omega+\kappa)}\right) \sin \kappa
$$




$$
\begin{gathered}
\Delta F_{2}=2 \Delta r\left(\sigma_{2}+r \frac{d \sigma_{2}}{d r}\right) \sin \kappa \\
\Delta F_{1}=2 \Delta r\left(\sigma_{1} \sin (\omega+\kappa) \cos \omega-\sigma_{2} \cos (\omega+\kappa) \sin \omega\right)
\end{gathered}
$$

Equation (A4.69) can be rearranged using trigonometric identities such that

$$
\Delta \mathrm{F}_{1}=2 \Delta \mathrm{r}\left(\frac{\sigma_{1}+\sigma_{2}}{2} \sin \kappa+\frac{\sigma_{1}-\sigma_{2}}{2} \sin (2 \omega+\kappa)\right)
$$

The force balance over the arched element is

$$
\Delta W+\Delta F_{2}=\Delta F_{1}
$$

Substituting equations (A4.67), (A4.68), and (A4.70) into the force balance given by equation (A4.71) yields an expression in $\sigma_{1}$ and $\sigma_{2}$. As for the passive external stress arch model, the criterion for the formation of a stable stress-free surface is given in the cohesive arching model by

$$
\sigma_{2}=0
$$

Therefore, a relationship is needed between $\sigma_{1}$ and $\sigma_{2}$ in order to express the force balance given by equation (A4.71) as a function of $\sigma_{2}$ only so that the arching criterion given by equation (A4.72) can then be applied. In the case of a cohesive material with an unconfined yield stress $f_{c}$ as shown in Fig. A4.4, the relationship between $\sigma_{1}$ and $\sigma_{2}$ is given by

$$
\sigma_{1}=\mathrm{f}_{\mathrm{c}}+\frac{1+\sin \delta}{1-\sin \delta} \sigma_{2}
$$

The development of this relationship is given below in Section A4.2.5.

In this work, the unconfined yield stress is produced by the application of an electric field. In Chapter 4, Fig. 4.14 shows that the unconfined yield stresses measured in the shear cell experiments were approximately linearly dependent on the field strength, $E_{a}$. Therefore,

$$
f_{c}=m E_{a}+b
$$

where $m$ and $b$ are constants fit from the experimental data. Since the glass spheres used in these experiments exhibit no cohesive behaviour 
without an applied electric field, the small non-zero intercept on the $y$ axis is an experimental artifact and $b$ can be taken to be essentially zero.

For the wedge shaped hopper used in the arching experiments with the electrodes on the side walls, the electric field strength is given by

$$
E_{a}=\frac{V}{2 \kappa r}
$$

Substituting equations (A4.67), (A4.68), (A4.70), (A4.73), (A4.74) and (A4.75) into the force balance equation (A4.71) results in the following differential equation:

$$
r \frac{d \sigma_{2}}{d r}-\sigma_{2} X=-\Gamma r Y+Z r^{-1}+Z_{0}
$$

where

$$
\begin{aligned}
& X=\frac{\sin \delta}{1-\sin \delta}\left(1+\frac{\sin (2 \omega+\kappa)}{\sin \kappa}\right) \\
& Y=\left(\frac{(\omega+\kappa) \sin \kappa}{\sin ^{2}(\omega+\kappa)}+\frac{\sin \omega}{\sin (\omega+\kappa)}\right) \\
& Z=\frac{m V}{2 \kappa}\left(\frac{1}{2}+\frac{1}{2} \frac{\sin (2 \omega+\kappa)}{\sin \kappa}\right) \\
& Z_{0}=b\left(\frac{1}{2}+\frac{1}{2} \frac{\sin (2 \omega+\kappa)}{\sin \kappa}\right)
\end{aligned}
$$

Solving differential equation (A4.76) using the boundary condition $\sigma_{2}(r)=$ $\sigma_{2}(\mathrm{H})$ at $\mathrm{r}=\mathrm{H}$ results in the final equation for $\sigma_{2}$ as

$\sigma_{2}(r)=-\frac{\Gamma Y r}{1-X}-\frac{Z^{-1}}{1+X}-\frac{Z_{0}}{X}+\left(\frac{\Gamma}{H}\right)^{X}\left(\sigma_{2}(H)+\frac{\Gamma Y H}{1-X}+\frac{Z_{H}^{-1}}{1+X}+\frac{Z_{0}}{X}\right)$

For an arch to form, $\sigma_{2}=0$. Setting equation (A4.81) equal to zero and solving for the critical value of $Z$ for arching, $Z_{c}$, gives

$$
Z_{c}=\frac{\frac{\Gamma Y}{1-X}\left[\left(\frac{r}{H}\right)^{X} H-r\right]+\frac{Z_{o}}{X}\left[\left(\frac{r}{H}\right)^{X}-1\right]+\left(\frac{r}{H}\right)^{X} \sigma_{2}(H)}{\left[\frac{r^{-1}}{1+X}-\left(\frac{r}{H}\right)^{X} \frac{H^{-1}}{1+X}\right]}
$$


The value of $Z_{c}$ is then used to calculate the holding voltage, $V_{h}$, by rearranging equation (A4.79) such that

$$
V_{h}=\frac{2 \kappa Z_{c}}{m}\left(\frac{1}{2}+\frac{1}{2} \frac{\sin (2 \omega+\kappa)}{\sin \kappa}\right)^{-1}
$$

\section{A4.2.5 RELATIONSHIP BETWEEN MAJOR AND MINOR PRINCIPAL STRESSES FOR COHESIVE MATERIAL}

The relationship between $\sigma_{1}$ and $\sigma_{2}$ for a cohesive material can be developed from geometrical considerations from Fig. A4.4. Figure A4.4 shows the internal yield locus for a cohesive material and two Mohr's circles. The Mohr's circles touch the yield locus because the material is considered to be at the point of incipient flow. The Mohr's circle which passes through the origin describes the stress state at a free surface where the minor principal stress is zero. The major principal stress in this case is called the unconfined yield stress, $f_{c}$. The other Mohr's circle represents an arbitrary stress state in the bulk of the material. The major and minor principal stresses in the material can be related through the unconfined yield stress. From Fig. A4.4,

$$
\begin{gathered}
P_{1}=\frac{f_{c}}{2}\left(\frac{1-\sin \delta}{\sin \delta}\right) \\
R_{m}=\frac{\sigma_{1}-\sigma_{2}}{2} \\
R_{m}=P_{2} \sin \delta \\
P_{2}=P_{1}+\sigma_{2}+R_{m} \\
\sigma_{1}=P_{2}+R_{m}-P_{1}
\end{gathered}
$$

Substituting equations (A4.86) and (A4.87) into equation (A4.88) gives

$$
\sigma_{1}=\sigma_{2}(1+\sin \delta)+R_{m}(1+\sin \delta)+P_{1} \sin \delta
$$

Substituting equations (A4.84) and (A4.85) into equation (A4.89) gives the expression which relates the major and minor principal stresses for use in the cohesive arching model. This expression is 


$$
\sigma_{1}=f_{c}+\frac{1+\sin \delta}{1-\sin \delta} \sigma_{2}
$$




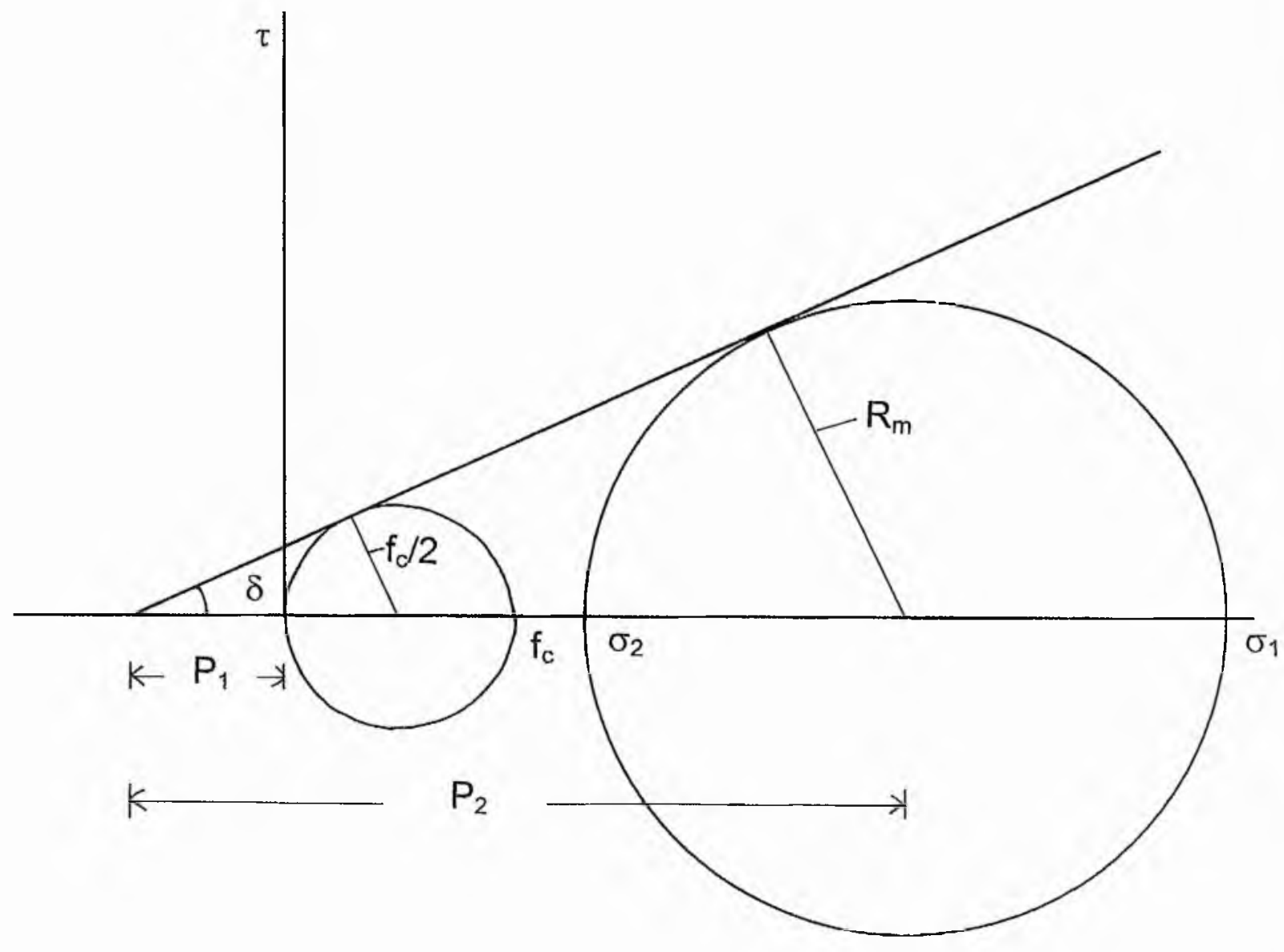

Fig. A4.4 Representation of internal yield locus and Mohr's stress circles for cohesive material. 
Table 3.1 Electrical stress and electrical cohesion from experimental wall and internal yield loci.

\begin{tabular}{|c|c|c|c|c|c|}
\hline \multicolumn{3}{|c|}{ Wall shear } & \multicolumn{3}{c|}{ Internal shear } \\
\hline Electric field & $\sigma_{\mathrm{e}}$ & $c_{\mathrm{e}}$ & Electric field & $\sigma_{\mathrm{e}}$ & $\mathrm{c}_{\mathrm{e}}$ \\
$\mathrm{kV} \mathrm{m}^{-1}$ & $\mathrm{~N} \mathrm{~m}^{-2}$ & $\mathrm{~N} \mathrm{~m}^{-2}$ & $\mathrm{kV} \mathrm{m}$ & $\mathrm{N} \mathrm{m}^{-2}$ & $\mathrm{~N} \mathrm{~m}^{-2}$ \\
\hline 0 & 0 & 0 & 0 & 0 & 0 \\
390 & 72 & 18 & 470 & 385 & 133 \\
790 & 203 & 56 & 800 & 703 & 246 \\
\hline
\end{tabular}


Table 3.2 Electrical properties of materials, measured in situ across electrodes in rectangular duct.

\begin{tabular}{|l|c|c|c|}
\hline Material & $\begin{array}{c}\text { Particle size } \\
\mathrm{mm}\end{array}$ & $\begin{array}{c}\text { Res i stance } \\
\mathrm{M} \Omega\end{array}$ & $\begin{array}{c}\text { Capacitance } \\
\mathrm{pF}\end{array}$ \\
\hline $\begin{array}{l}\text { Turnip } \\
\text { seeds }\end{array}$ & $1.2-1.7$ & 48 & 15 \\
\hline Sand & $0.6-0.85$ & 43 & 12 \\
\hline $\begin{array}{c}\text { NaCl salt } \\
\text { without anti- } \\
\text { caking agent) }\end{array}$ & $0.35-0.6$ & 14 & 14 \\
\hline
\end{tabular}


Table 3.3 Response of EVS system to voltage step changes.

\begin{tabular}{|c|c|c|c|c|}
\hline $\begin{array}{c}\text { Voltage step } \\
\mathrm{kV}\end{array}$ & \multicolumn{2}{|c|}{ Vol tage decrease } & \multicolumn{2}{l|}{ Vol tage increase } \\
\hline & $\begin{array}{c}\text { Transportation } \\
\text { time, s }\end{array}$ & $\begin{array}{c}\text { We ighing } \\
\text { time, s }\end{array}$ & $\begin{array}{c}\text { Transportation } \\
\text { time, s }\end{array}$ & $\begin{array}{c}\text { We i ghing } \\
\text { time, s }\end{array}$ \\
\hline $3 \Leftrightarrow 7$ & 0.3 & 0.4 & 0.5 & 0.7 \\
$1 \Leftrightarrow 7$ & 0.3 & 0.3 & 0.3 & 1.2 \\
\hline
\end{tabular}


Table 4.1 Electrical clamping stress predictions for 390 and $790 \mathrm{kV} \mathrm{m}^{-1}$ electric fields.

\begin{tabular}{|c|c|c|c|c|c|c|}
\hline & \multicolumn{3}{|c|}{$390 \mathrm{kV} \mathrm{m}-1$} & \multicolumn{3}{|c|}{$790 \mathrm{kV} \mathrm{m}^{-1}$} \\
\hline & $\begin{array}{l}\text { Fixed } \\
\text { Cap }\end{array}$ & $\begin{array}{c}\text { Elastic } \\
\text { Cap }\end{array}$ & $\begin{array}{l}\text { Combined } \\
\text { Force }\end{array}$ & $\begin{array}{l}\text { Fi xed } \\
\text { Cap }\end{array}$ & $\begin{array}{c}\text { Elastic } \\
\text { Cap }\end{array}$ & $\begin{array}{c}\text { Combined } \\
\text { Force }\end{array}$ \\
\hline $\begin{array}{l}\text { Mechanical force } \\
\text { per contact, } \mathrm{mN}\end{array}$ & $\begin{array}{c}0.085 / \\
0.26\end{array}$ & & $\begin{array}{c}0.085 / \\
0.26\end{array}$ & $\begin{array}{c}0.085 / \\
0.26\end{array}$ & & $\begin{array}{l}0.085 / \\
0.26\end{array}$ \\
\hline $\begin{array}{l}\text { Electrical force } \\
\text { per contact, } \mathrm{mN}\end{array}$ & $\begin{array}{r}3.8 / \\
2.0\end{array}$ & 0.97 & $\begin{array}{r}0.94 / \\
0.89\end{array}$ & $\begin{array}{l}16 / \\
8.4\end{array}$ & 2.4 & $\begin{array}{r}2.4 / \\
2.3\end{array}$ \\
\hline $\begin{array}{l}\text { Contact cap } \\
\text { radius, } \mu \mathrm{m}\end{array}$ & $\begin{array}{c}0.77 / \\
1.1\end{array}$ & 1.7 & $\begin{array}{r}1.8 / \\
1.8\end{array}$ & $\begin{array}{c}0.77 / \\
1.1\end{array}$ & 2.3 & $\begin{array}{r}2.4 \prime \\
2.4\end{array}$ \\
\hline $\begin{array}{l}\text { Electrical } \\
\text { clamping } \\
\text { stress, } \mathrm{kN} \mathrm{m}^{-2}\end{array}$ & $\begin{array}{r}8.9 / \\
4.8\end{array}$ & 2.3 & $\begin{array}{r}2.2 / \\
2.1\end{array}$ & $\begin{array}{r}37 / \\
20\end{array}$ & 5.6 & $\begin{array}{r}5.6 / \\
5.5\end{array}$ \\
\hline
\end{tabular}

* Data shown for two mechanical normal stress values as:

results for $200 \mathrm{~N} \mathrm{~m}^{-2}$ / results for $600 \mathrm{~N} \mathrm{~m}^{-2}$. 
Table 4.2 Values of $c_{f}$ and $k$ fitted to Beverloo correlation for mesh flow from experimental data for sand.

\begin{tabular}{|c|c|c|c|}
\hline $\begin{array}{c}\text { Column size, } \\
\mathrm{mm}\end{array}$ & $\begin{array}{c}\text { Sand size, } \\
\mathrm{mm}\end{array}$ & $\mathrm{k}$ & $\mathrm{c}_{\mathrm{f}}$ \\
\hline \multirow{2}{*}{64} & $0.6-1.2$ & 2.9 & 0.60 \\
\hline \multirow{2}{*}{115} & $1.2-2.4$ & 2.0 & 0.49 \\
& $0.6-1.2$ & 3.1 & 0.94 \\
$1.2-2.4$ & 2.6 & 0.69 \\
\hline
\end{tabular}


Table 5.1 Comparison of electrical clamping forces determined from wall shear experiments and single contact experiments for a glass sphere of $330 \mu \mathrm{m}$ radius.

\begin{tabular}{|c|cc|c|}
\hline $\begin{array}{c}\text { Electric field, } \\
\mathrm{kV} \mathrm{m}\end{array}$ & $\begin{array}{c}\text { Electrical clamping force } \\
\text { from wal y y ield exp't, } \\
\mathrm{mN}\end{array}$ & $\begin{array}{c}\text { Electrical c lamping force } \\
\text { from single contact exp't, } \\
\mathrm{mN}\end{array}$ \\
\hline 390 & $\mathrm{~S}=2 \mathrm{R} \quad \mathrm{S}$ & 0.07 \\
790 & 0.03 & 0.07 & 0.14 \\
\hline
\end{tabular}


Table 5.2 Comparison of measured and best fit parameters in Sphere Resistance model equation (5.55).

\begin{tabular}{|c|c|c|c|c|}
\hline & \multicolumn{2}{|c|}{$\begin{array}{c}\text { Surface resistivity, } \rho_{\mathrm{s}} \\
\text { Tera } \Omega \mathrm{m} / \mathrm{m}\end{array}$} & \multicolumn{2}{c|}{$\begin{array}{c}\text { Sphere angle, } \theta \\
\text { deg }\end{array}$} \\
\hline $\begin{array}{c}\text { Specimen } \\
\text { no }\end{array}$ & Measured & Best fit & Measured & Best fit \\
\hline $1 \mathrm{H}$ & 1.5 & 125 & 89 & 0.33 \\
$3 \mathrm{H}$ & 1.5 & 16 & 122 & 0.51 \\
$4 \mathrm{H}$ & 1.5 & 13 & 112 & 0.37 \\
$1 \mathrm{~J}$ & 1.5 & 17 & 118 & 0.29 \\
$1 \mathrm{~K}$ & 1.5 & 75 & 117 & 0.28 \\
\hline
\end{tabular}



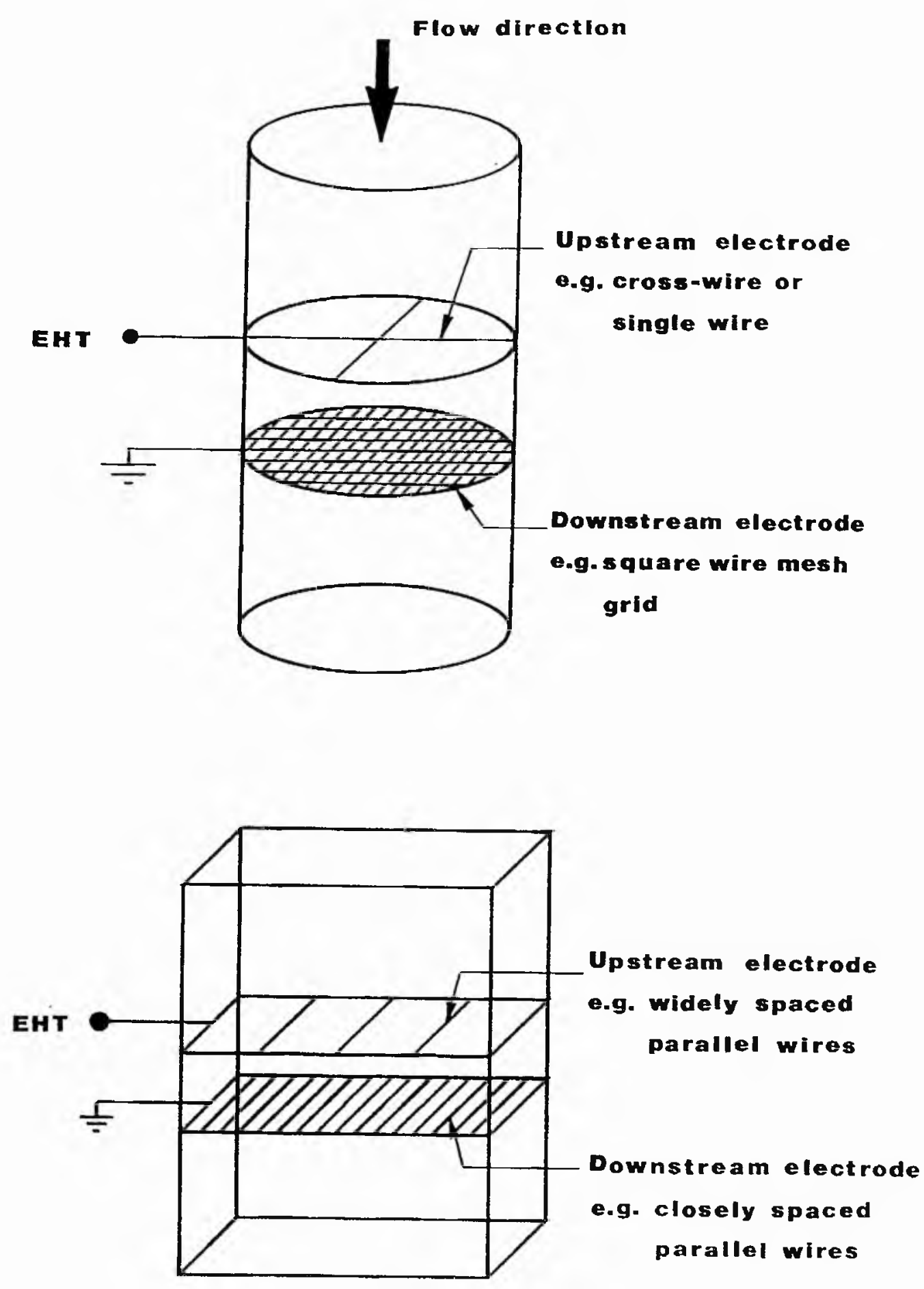

Fig. 1.1 Typical electrode configurations of the Electromechanical Valve for Solids in cylindrical and rectangular ducts. 


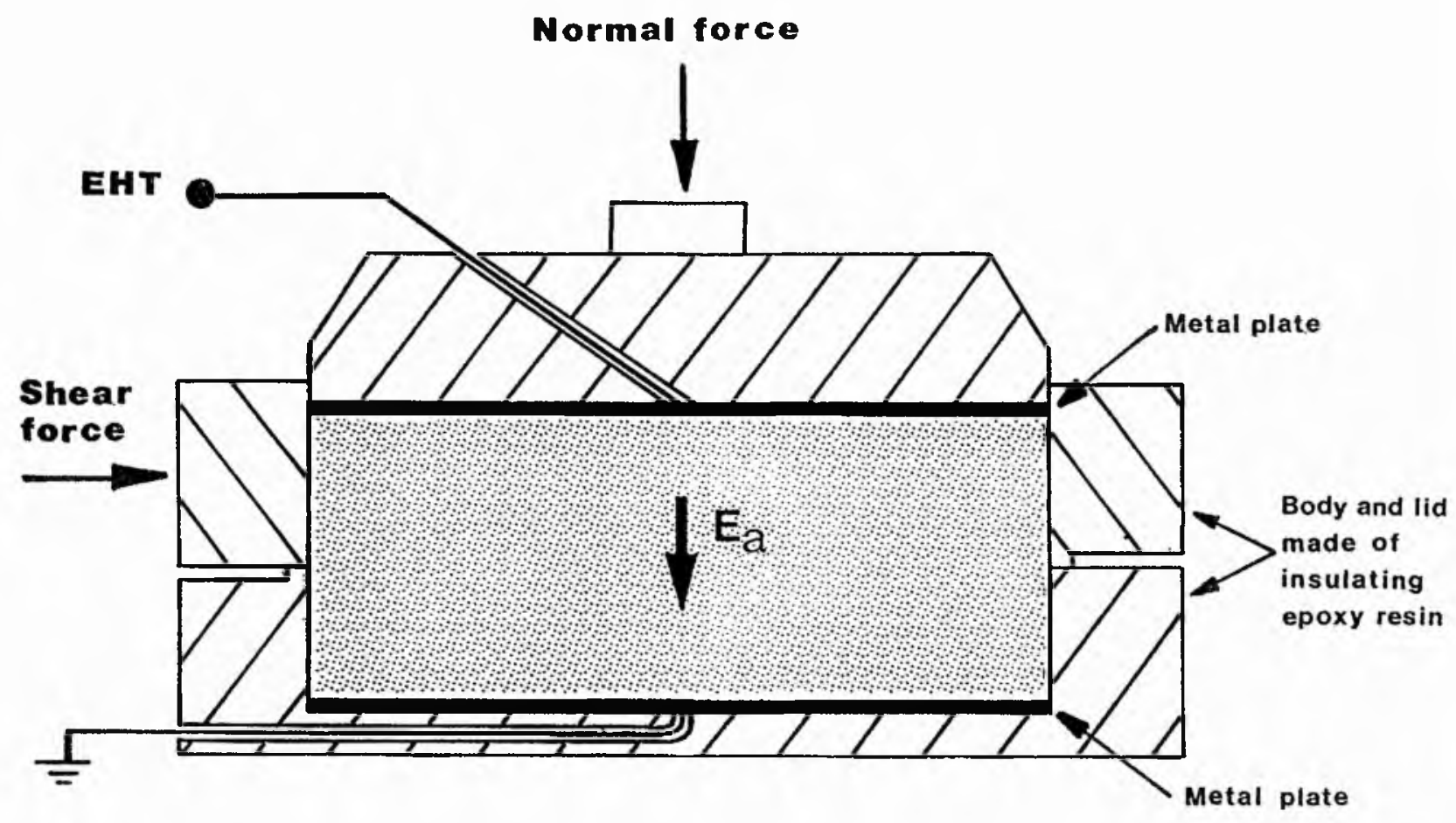

Fig. 3.1 Diagram of shear cell incorporating applied electric field. 


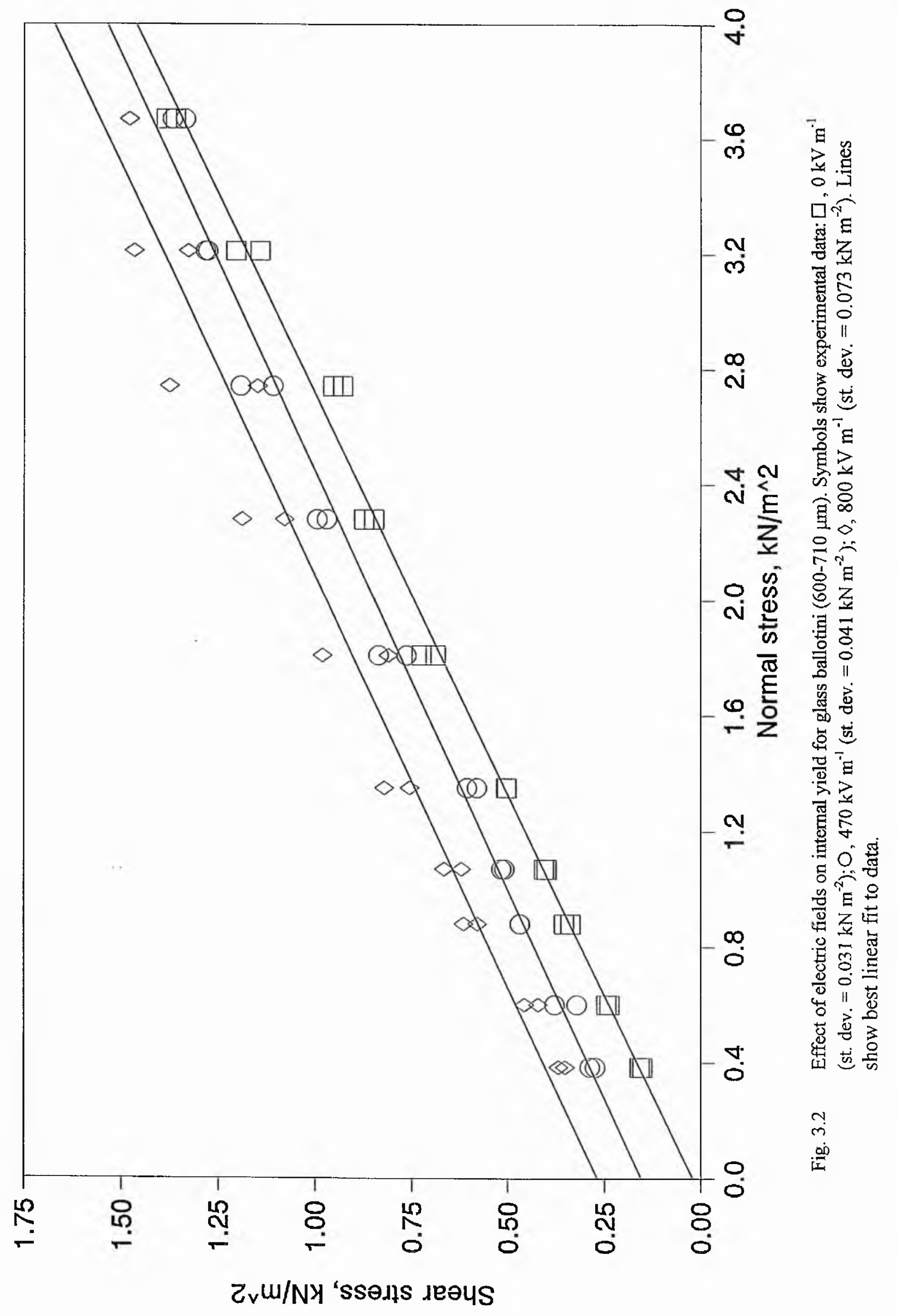




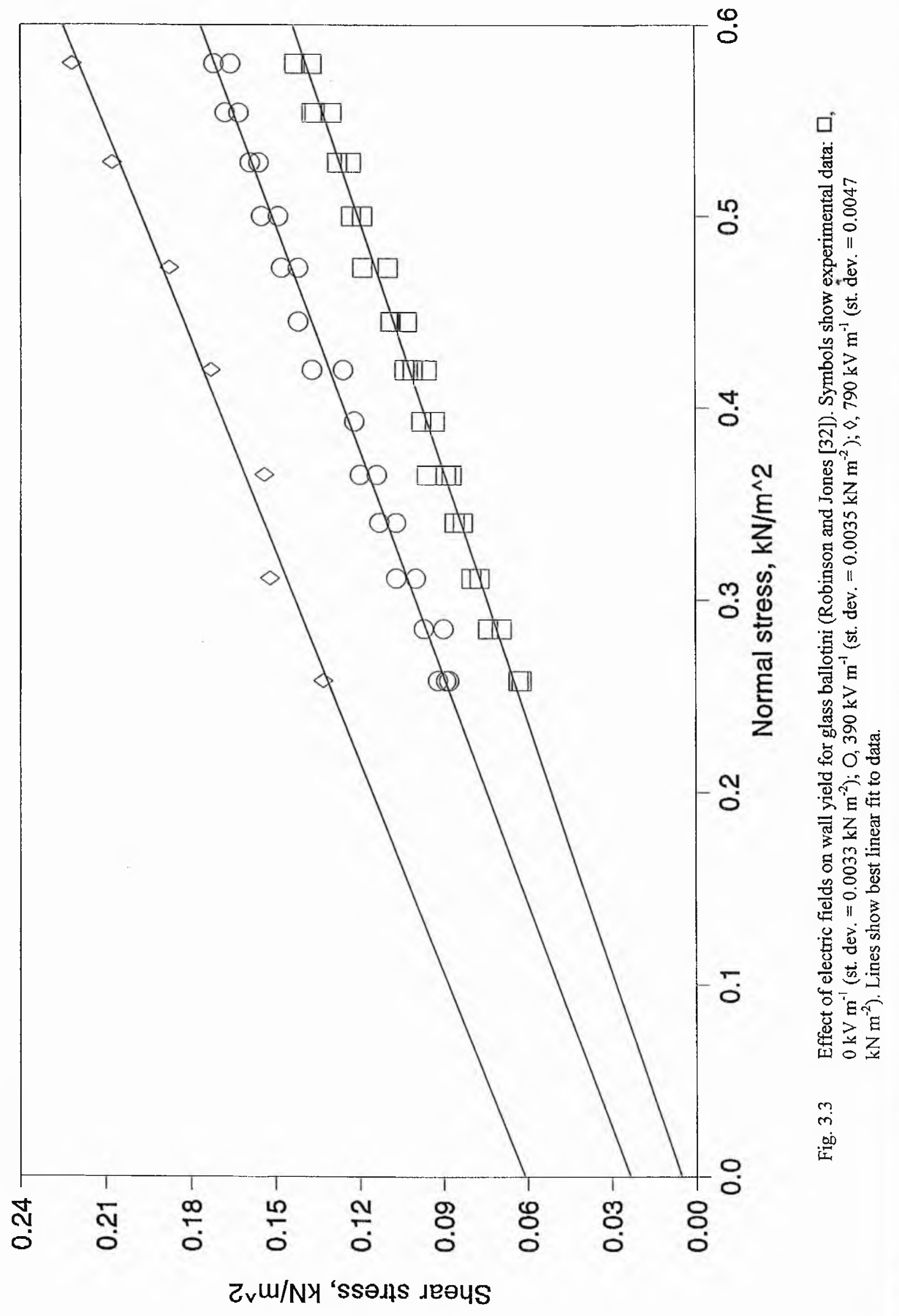




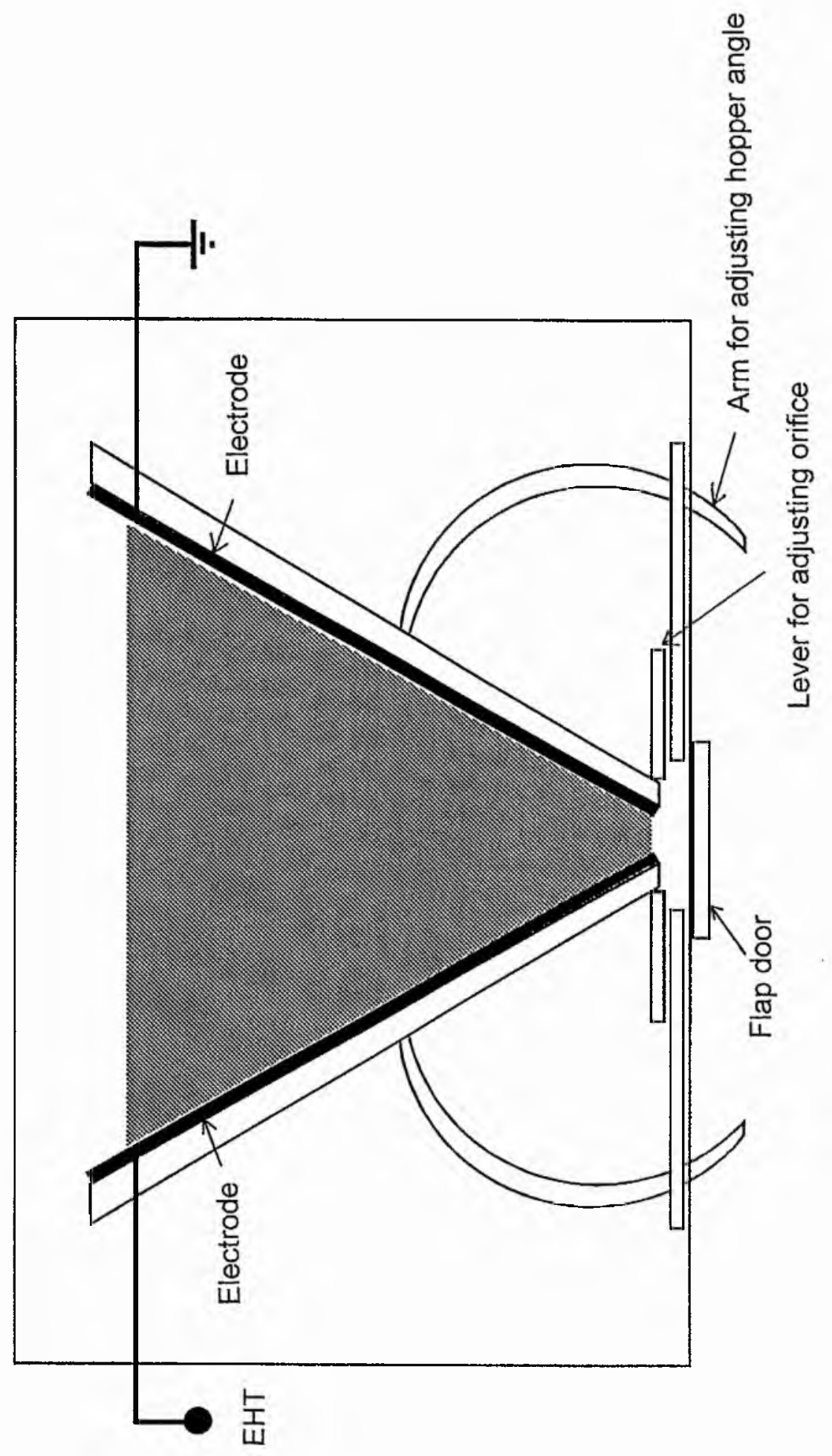

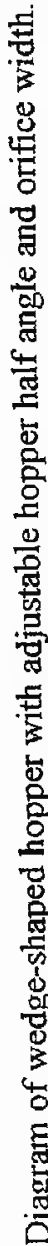

$\dot{r}$

in 


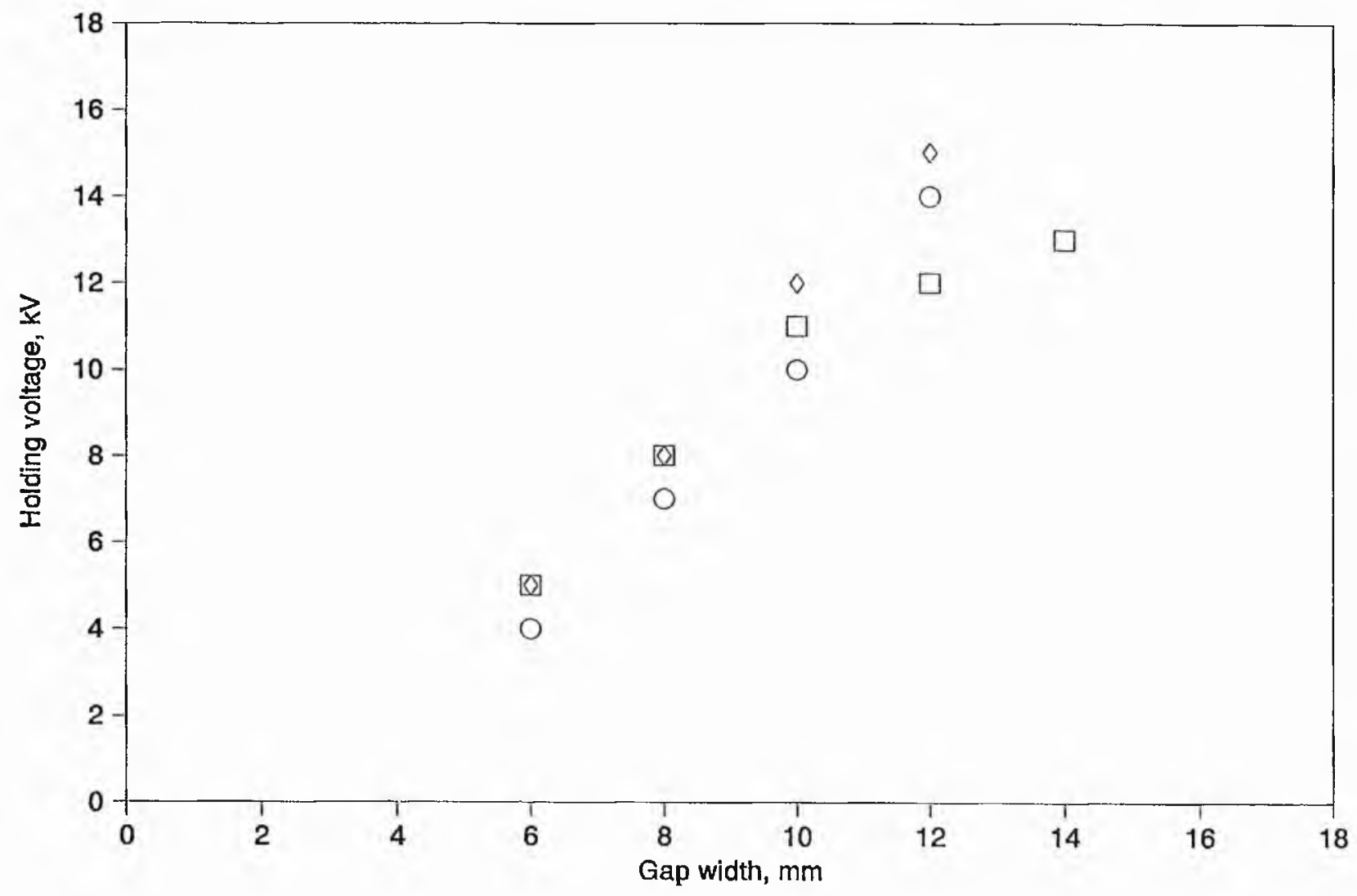

Fig. 3.5 Static holding voltages for glass ballotini $(600-710 \mu \mathrm{m})$ in smooth walled hopper for varying hopper half angle: $\square, 0^{\circ} ; 0,10^{\circ} ; \diamond, 20^{\circ}$.

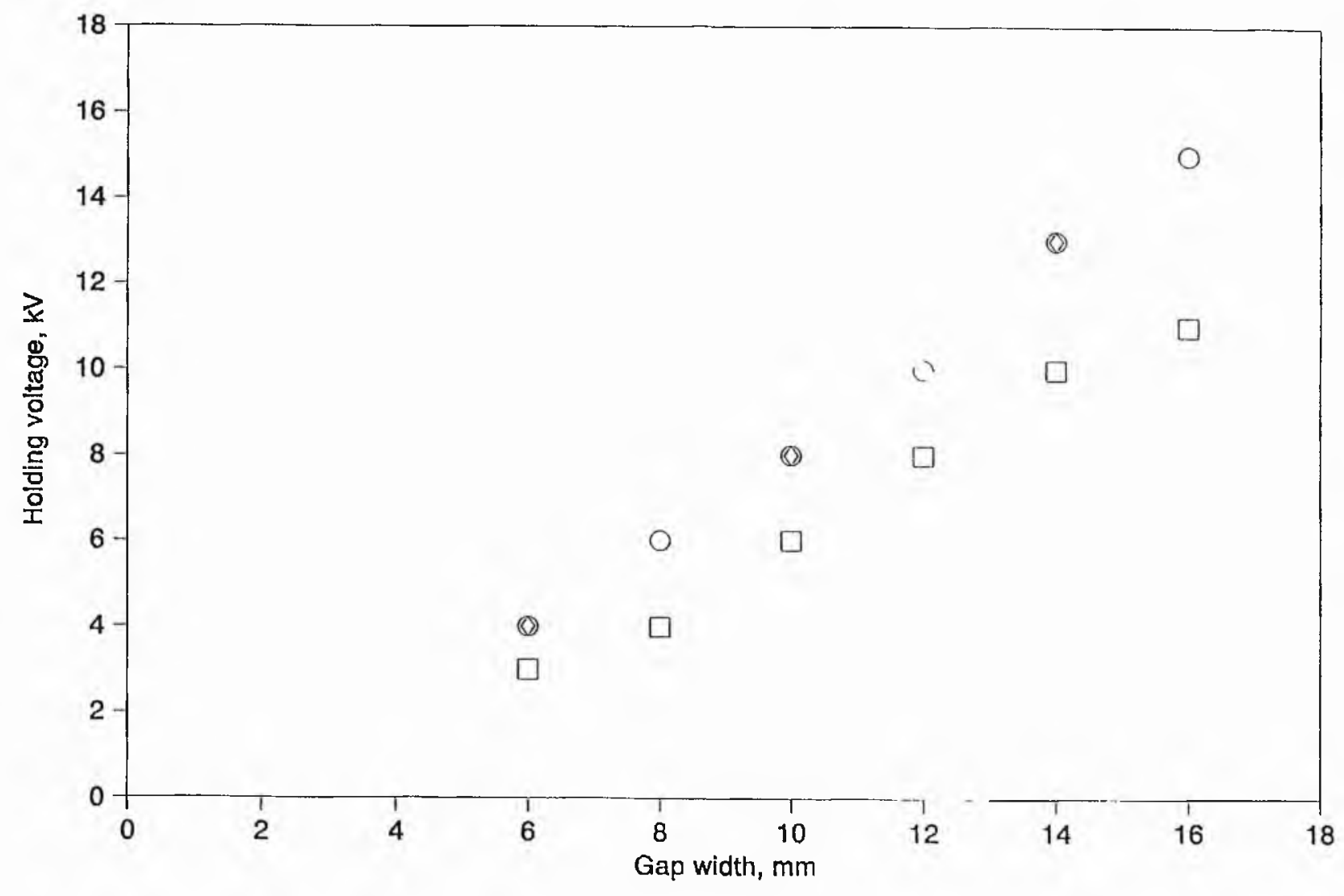

Fig. 3.6 Static holding voltages for glass ballotini $(600-710 \mu \mathrm{m})$ in rough walled hopper for varying hopper half angle: $\square, 0^{\circ} ; 0,10^{\circ}: 0,20^{\circ}$. 


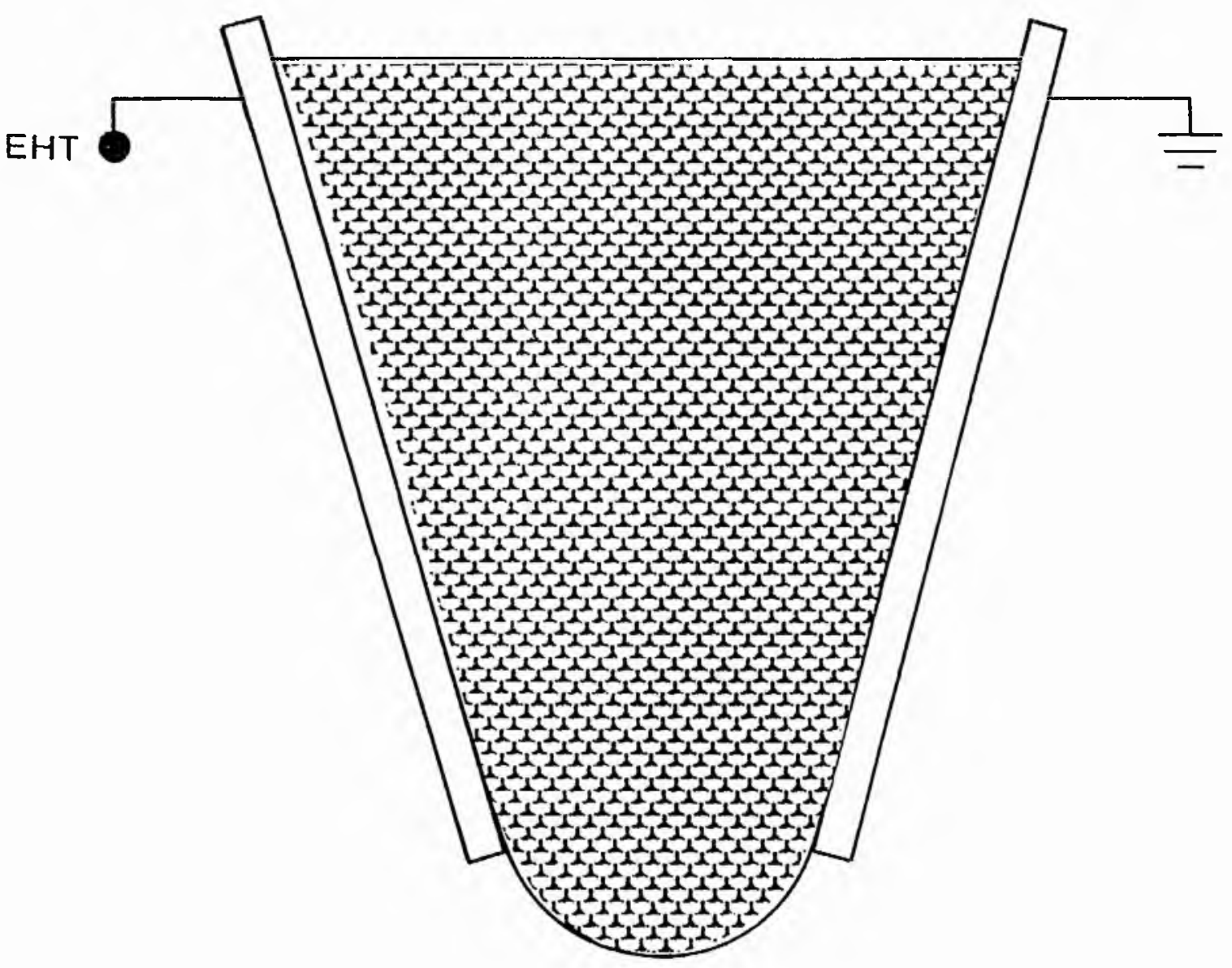

Fig. 3.7 Schematic diagram showing general shape of convex arch formed at orifice of wedge-shaped hopper when holding voltage is applied. 


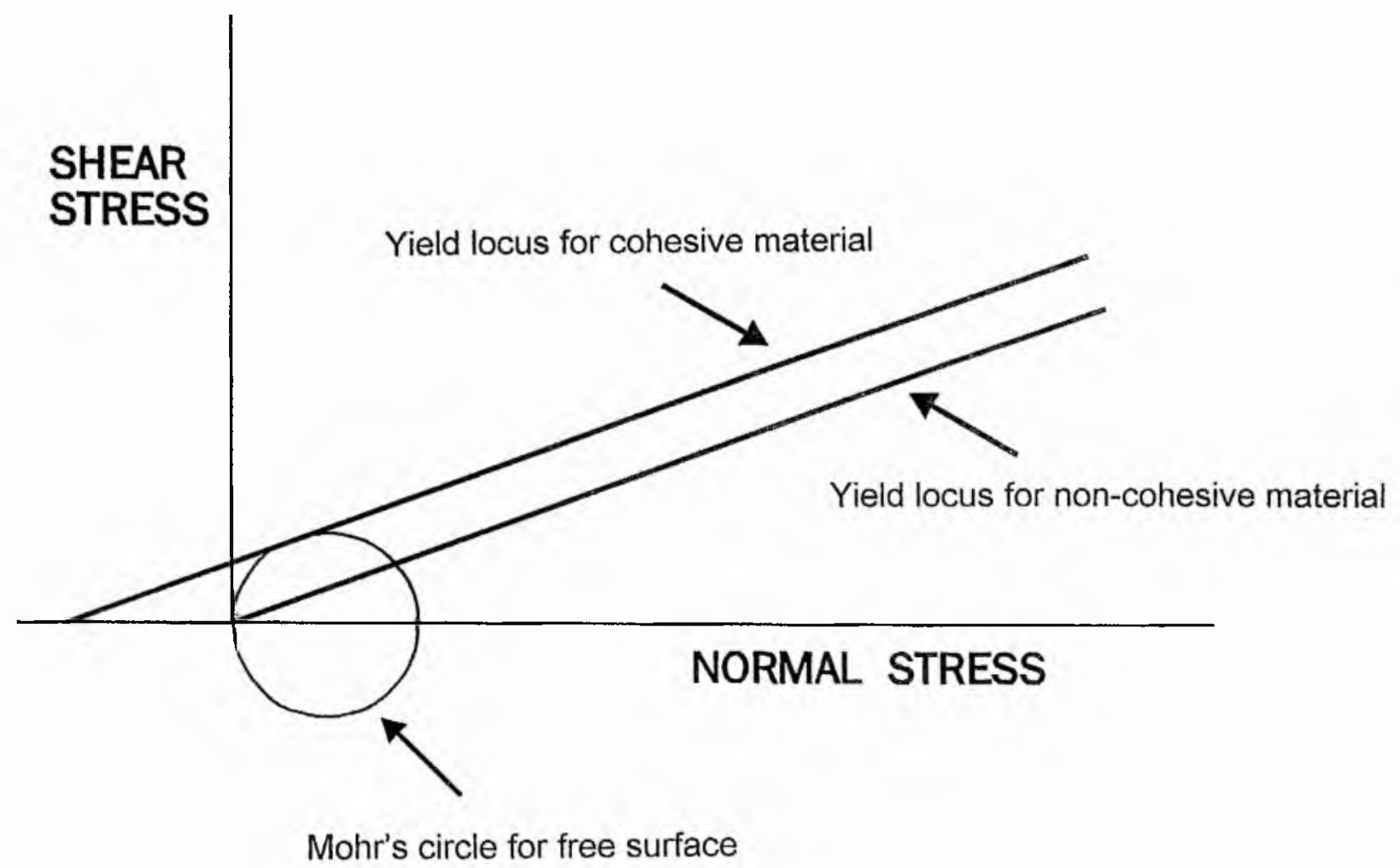

Fig. 3.8 Representation of Coulomb yield loci for cohesive and non-cohesive material with Mohr's stress circle for stable free surface. 


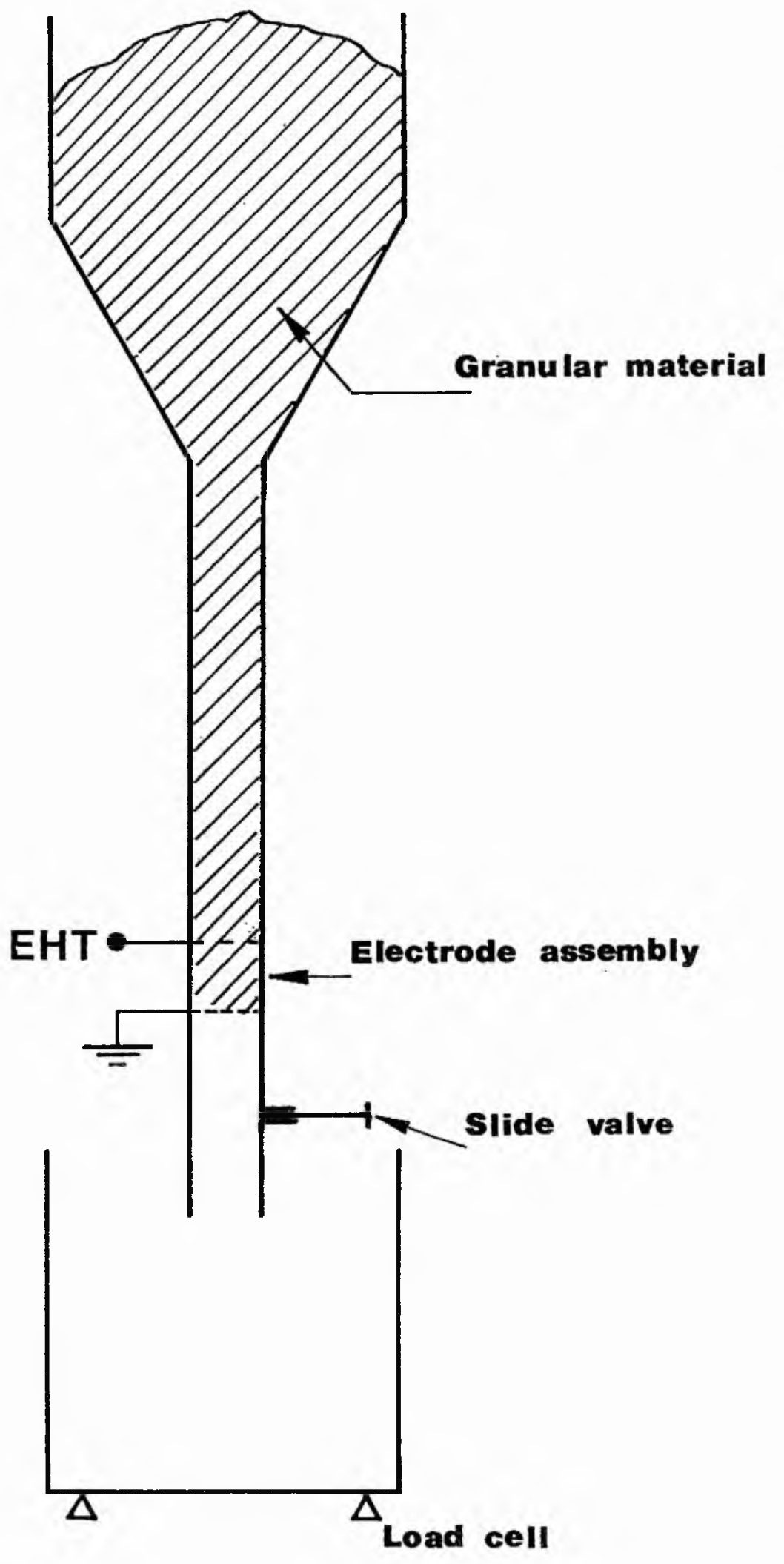

Fig. 3.9 Diagram of test rig for ineasuring flowrate through EVS. 


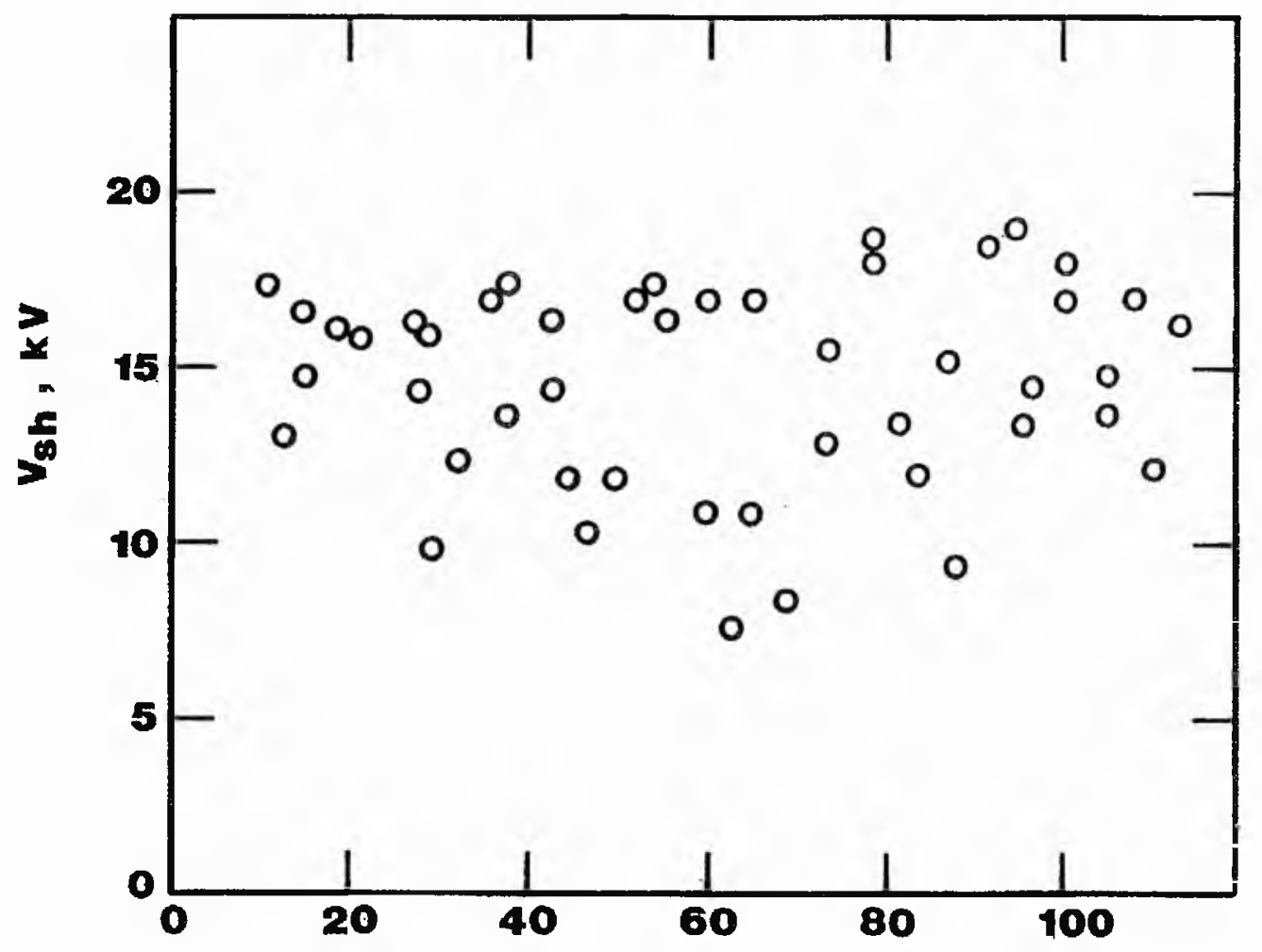

Height of sand above top electrode, $\mathrm{cm}$

Fig. 3.10 Effect of height of material on static holding voltage for 600-1200 $\mu \mathrm{m}$ sand in $115 \mathrm{~mm}$ column. Upper electrode $=$ cross-wire; lower electrode mesh opening

$=10 \mathrm{~mm}$; inter-electrode gap $($ IEG) $=36 \mathrm{~mm}$. 


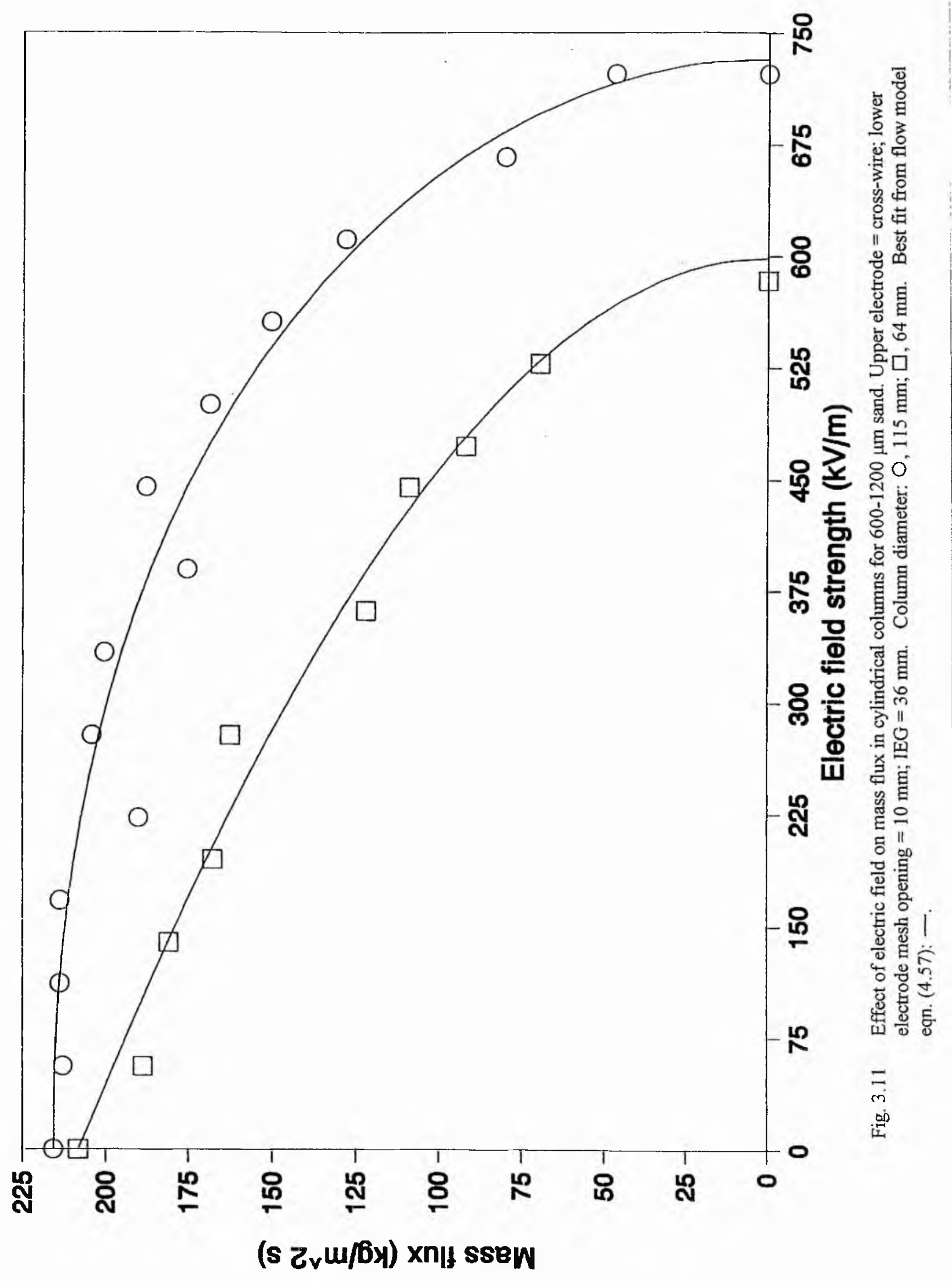




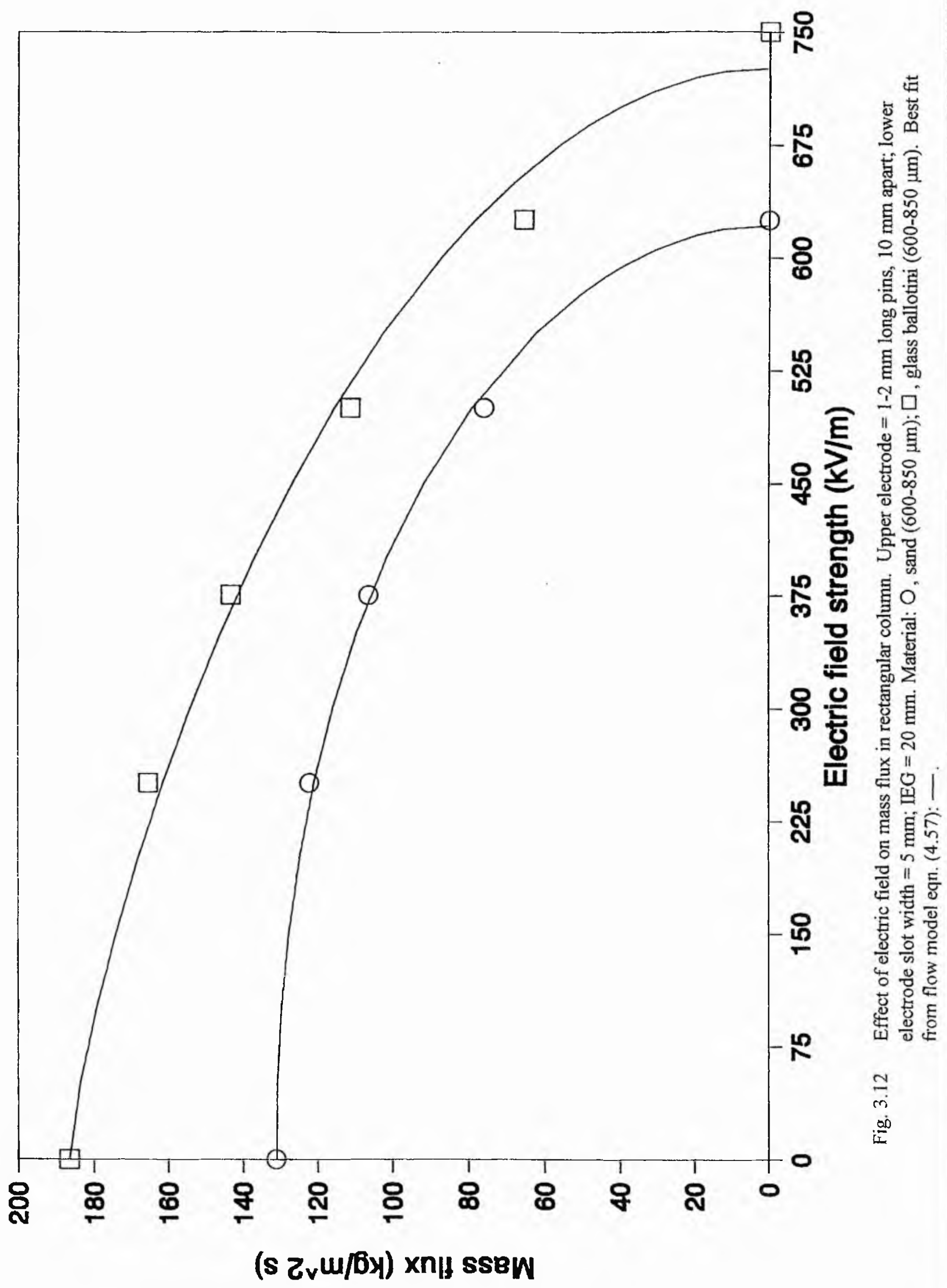




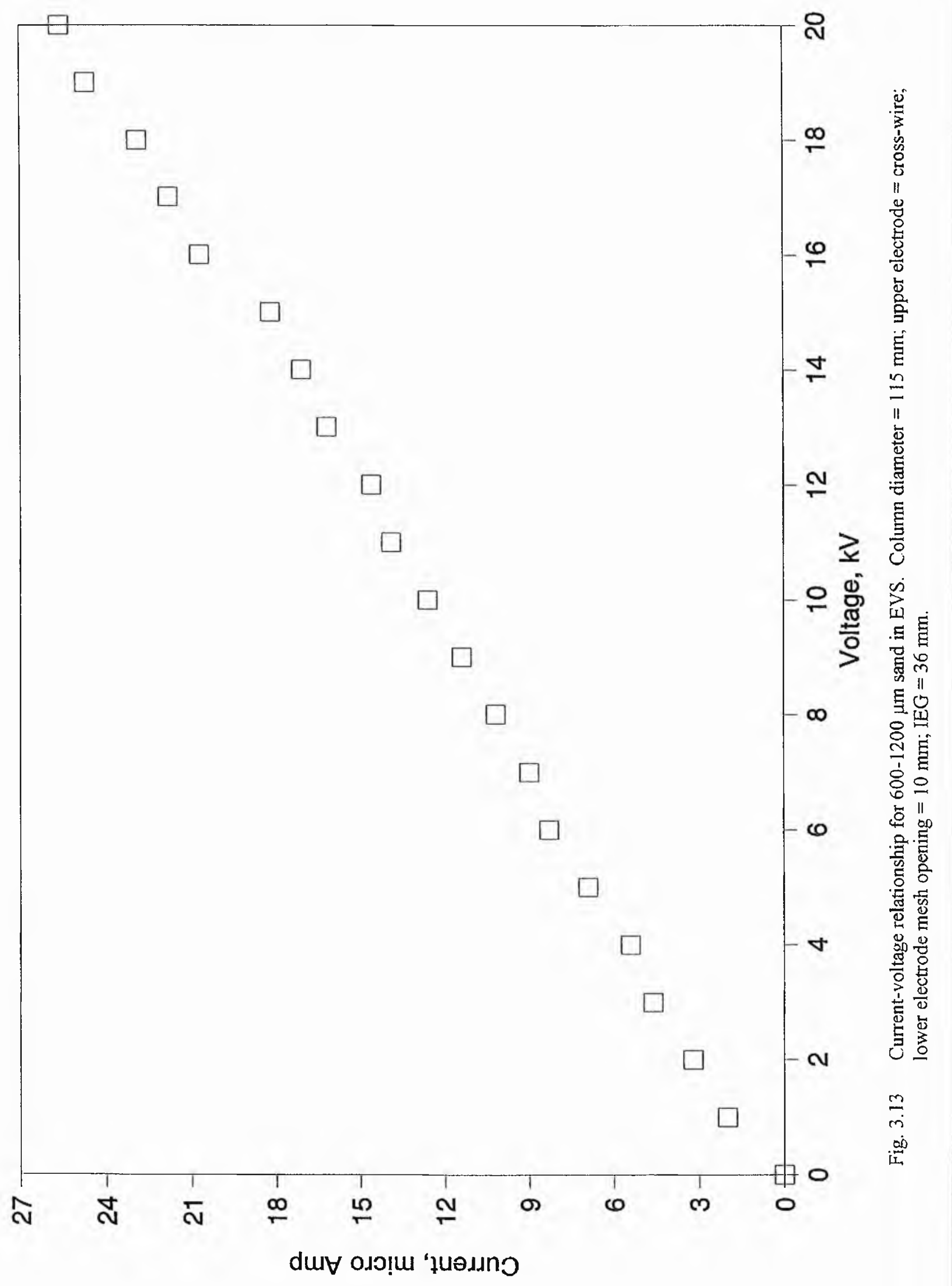




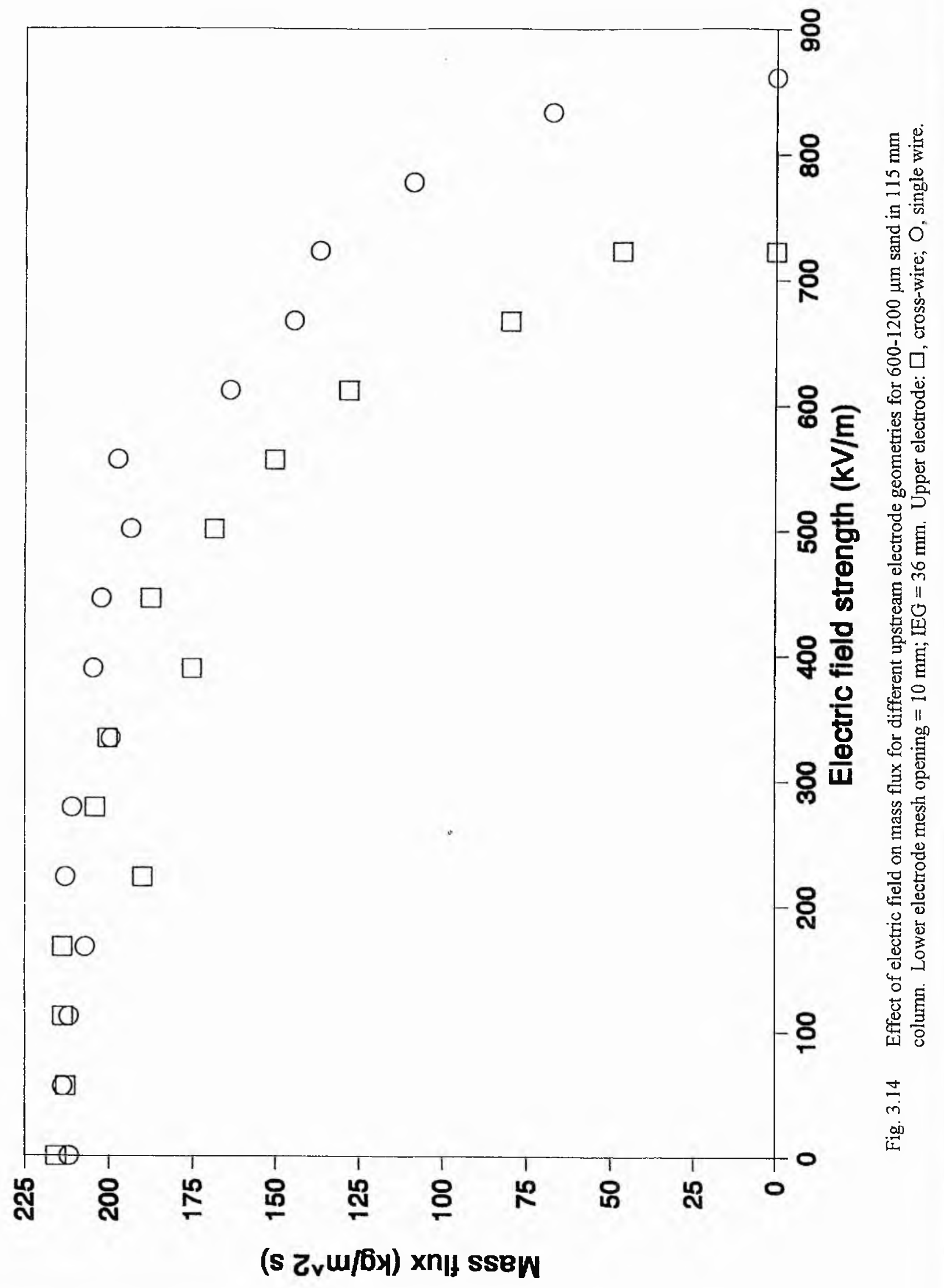




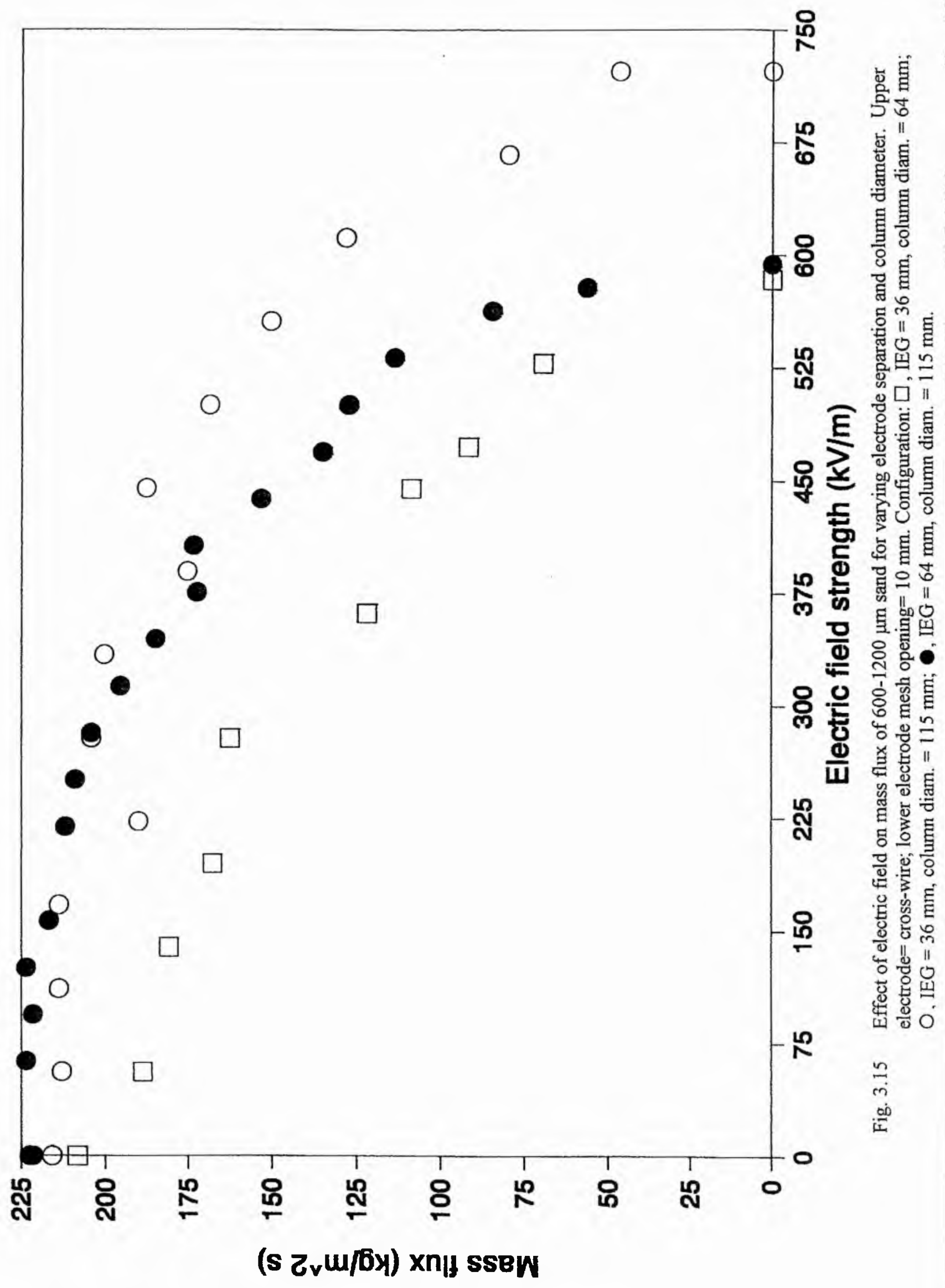




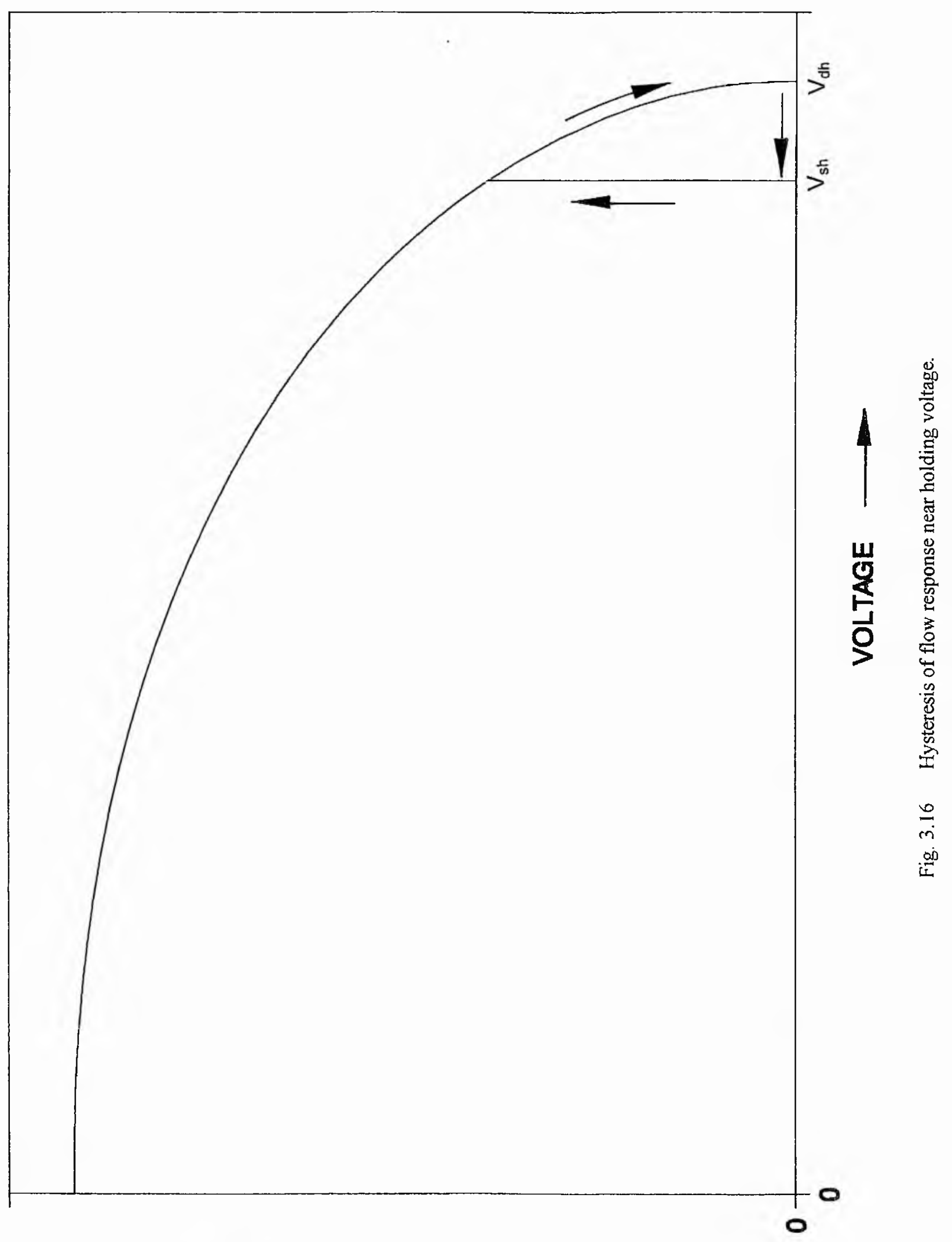




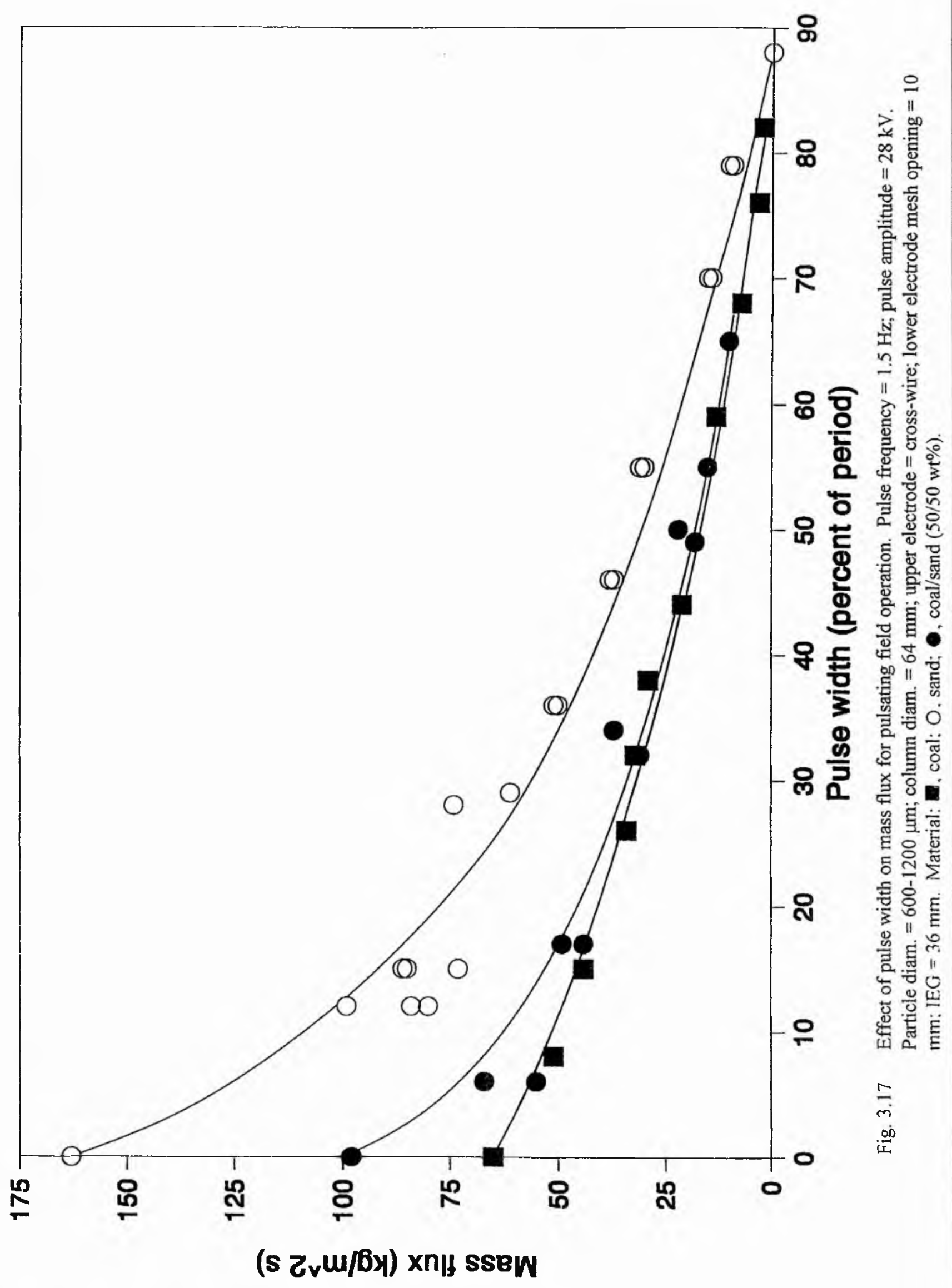




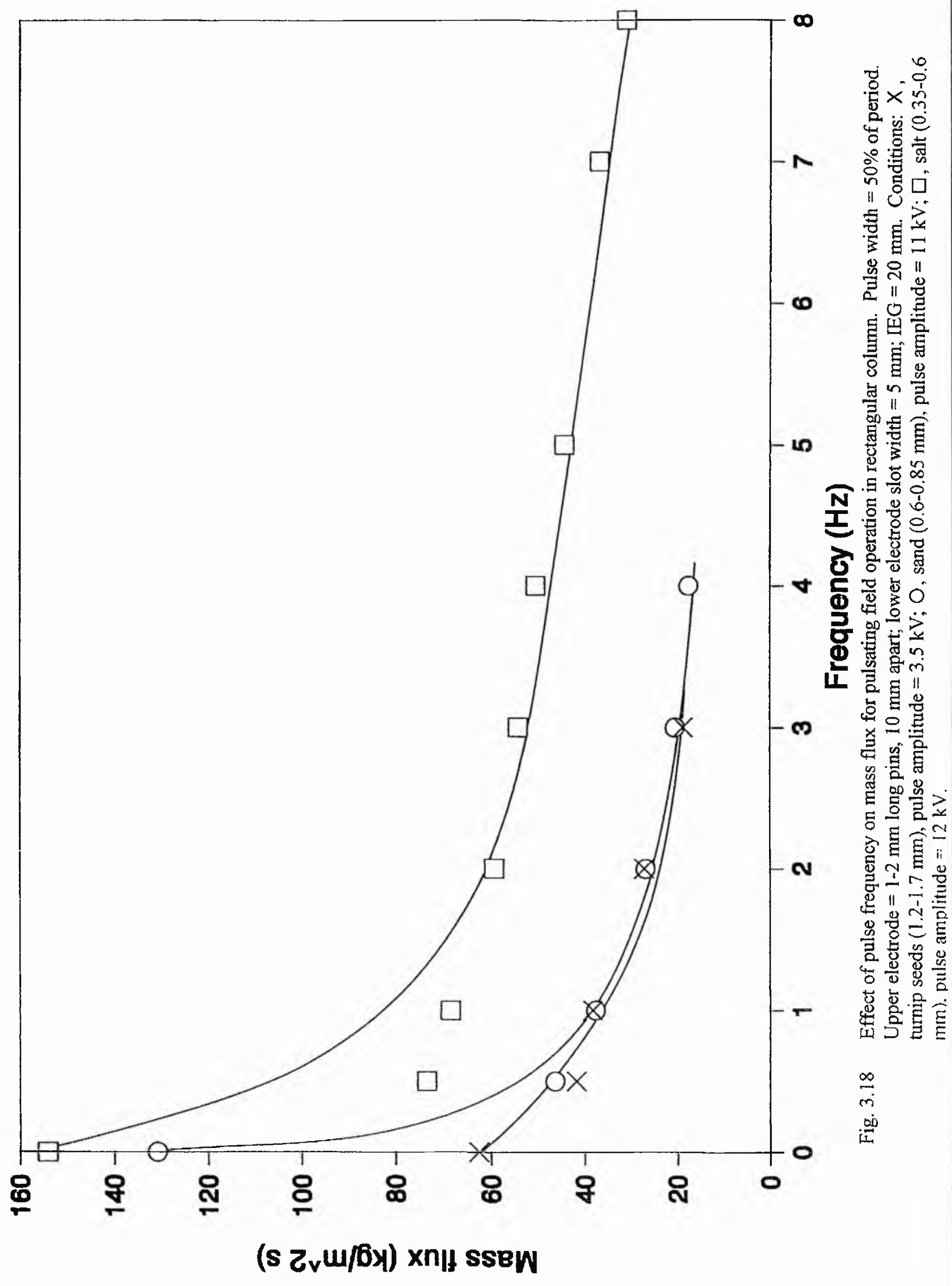




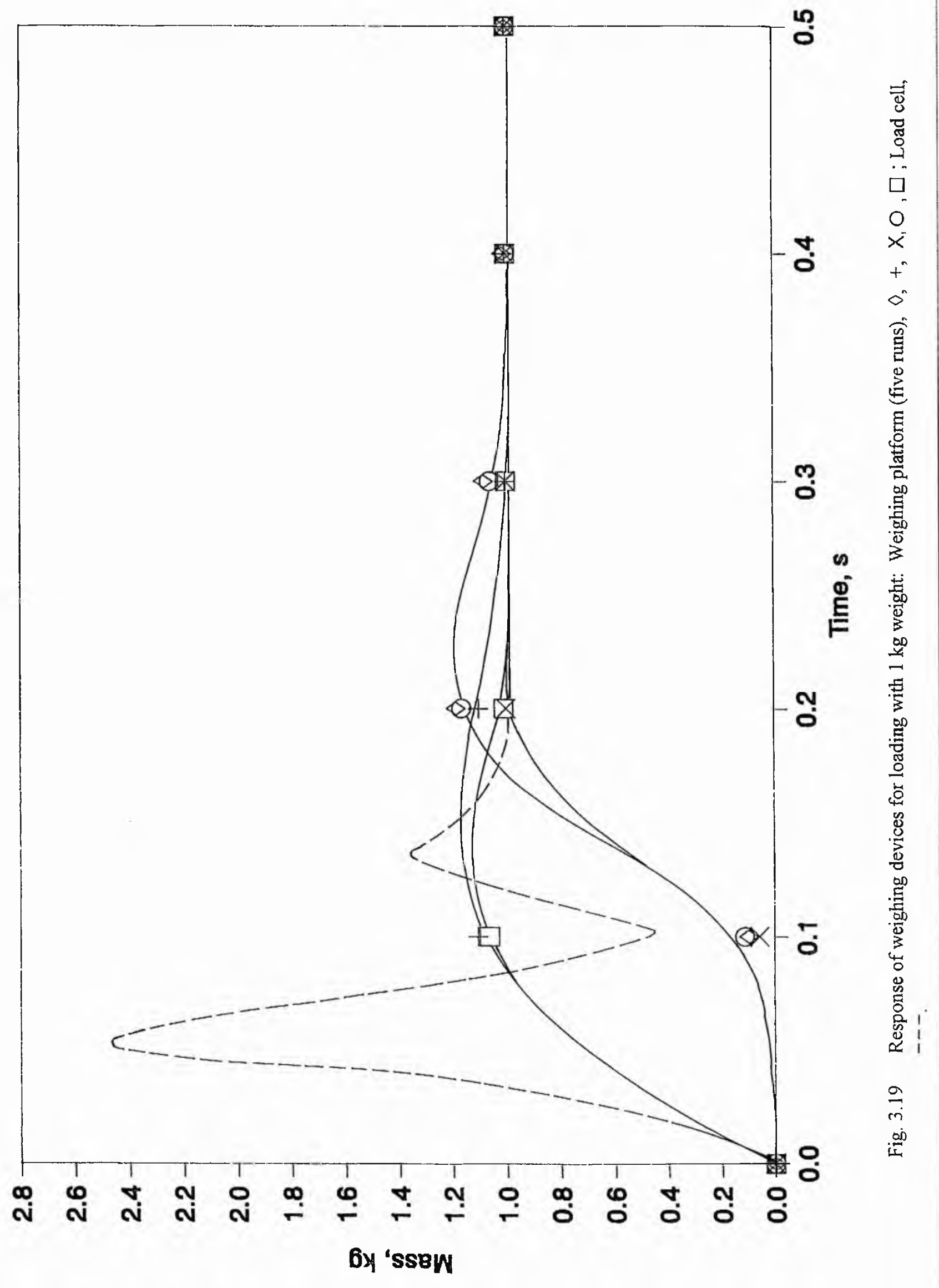




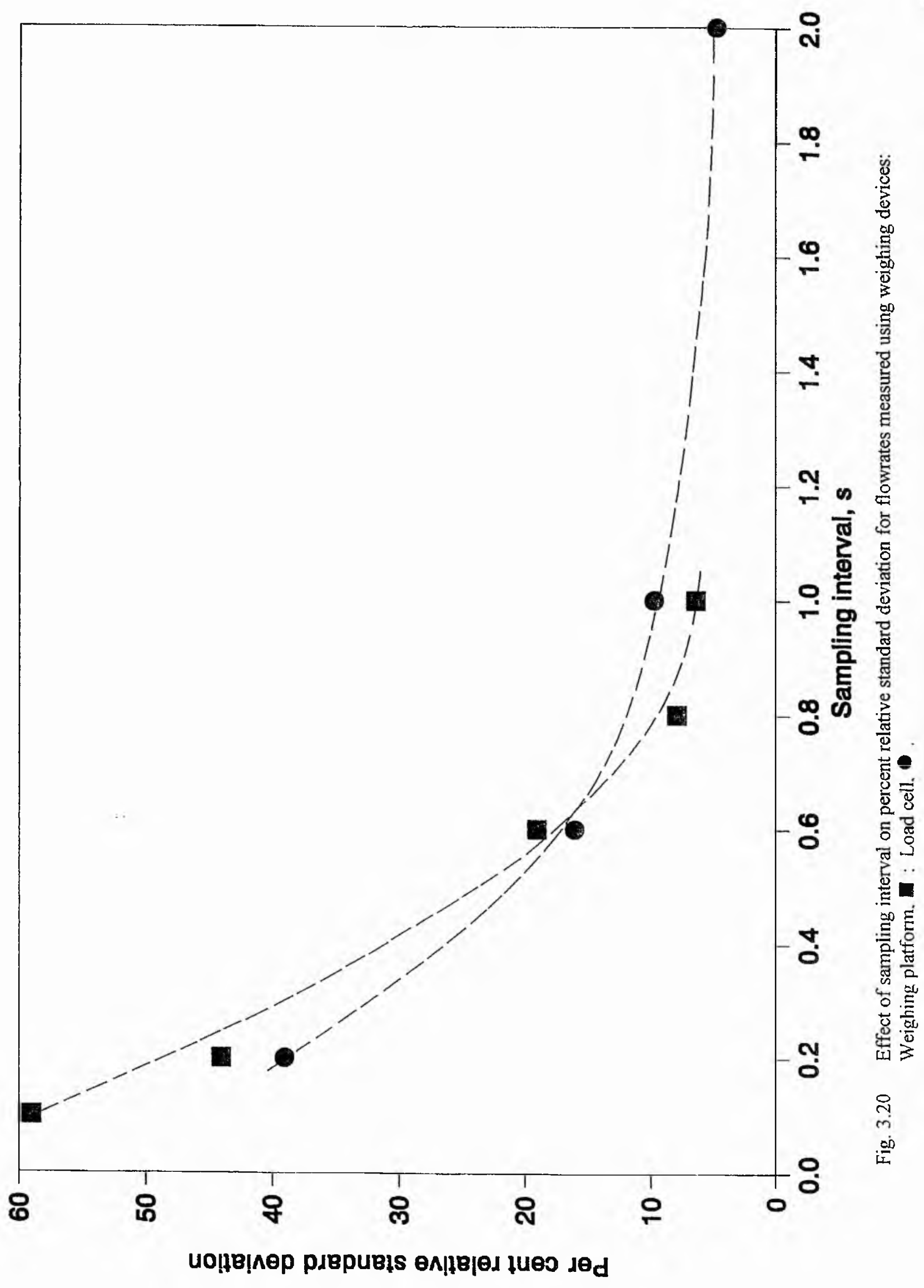




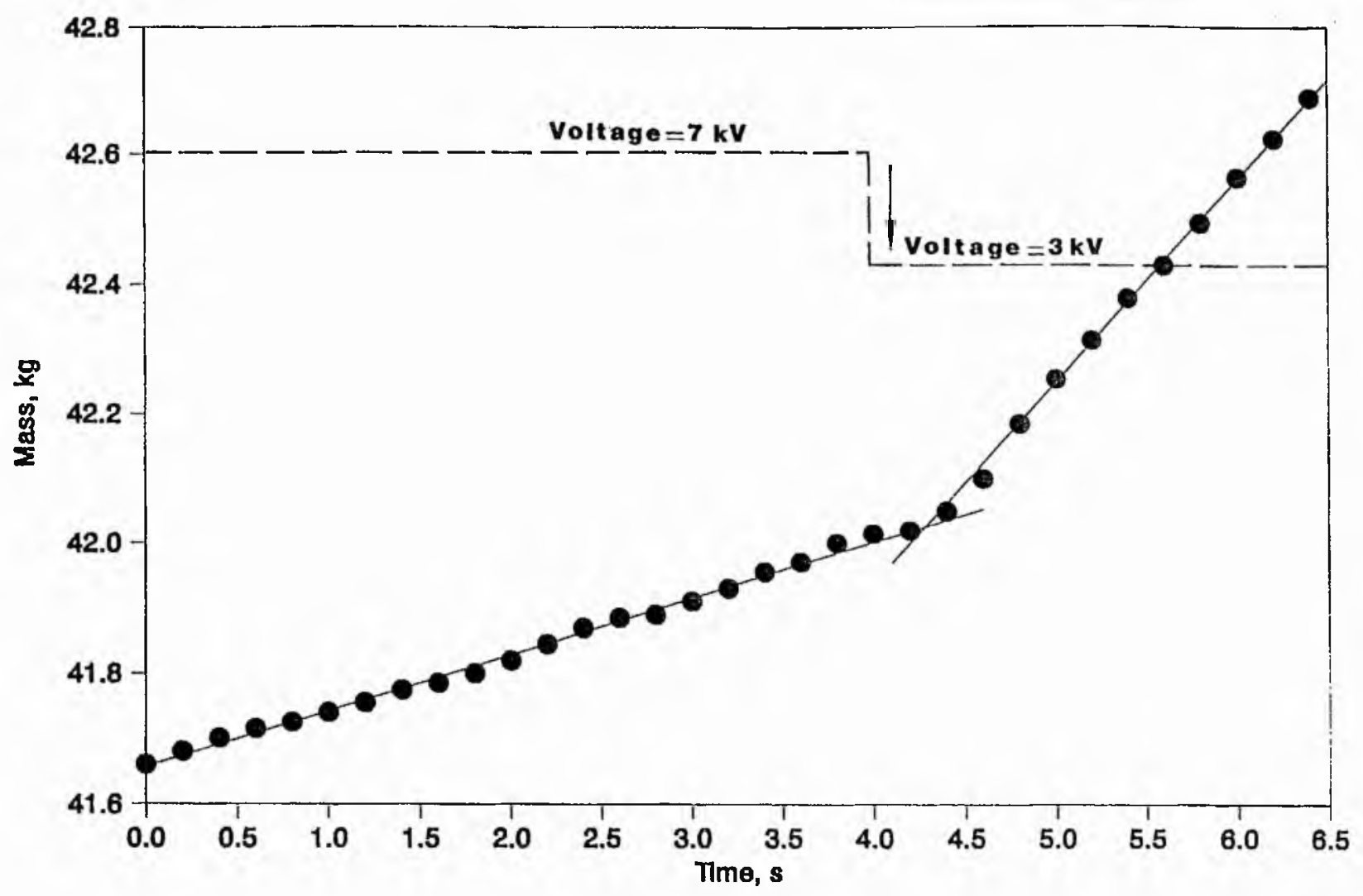

(a)

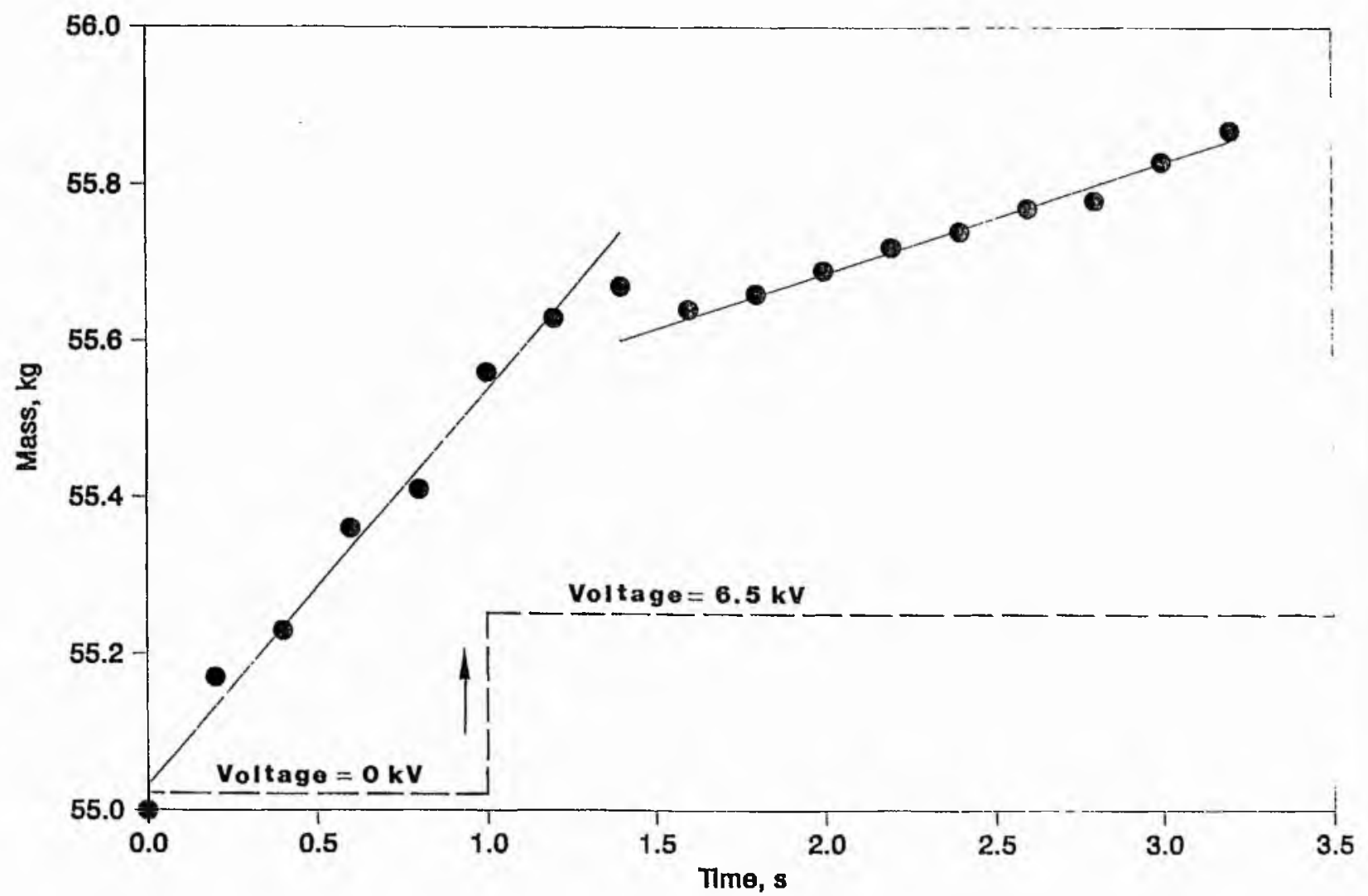

(b)

Fig. 3.21 (a) Response of load cell to step change in EVS voltage from 7 to $3 \mathrm{kV}$ made at $4.0 \mathrm{~s}$. Symbols show experimental data. Solid lines show best linear fits to data over ranges of 0.0-3.8 $\mathrm{s}$ and 5.2-6.4 s.

(b) Response of weighing platform to step change in EVS voltage from 0 to $6.5 \mathrm{kV}$ made at $1.0 \mathrm{~s}$. Symbols show experimental data. Solid lines show best linear fits to data over ranges of $0.0-0.8 \mathrm{~s}$ and $2.0-3.2 \mathrm{~s}$. 


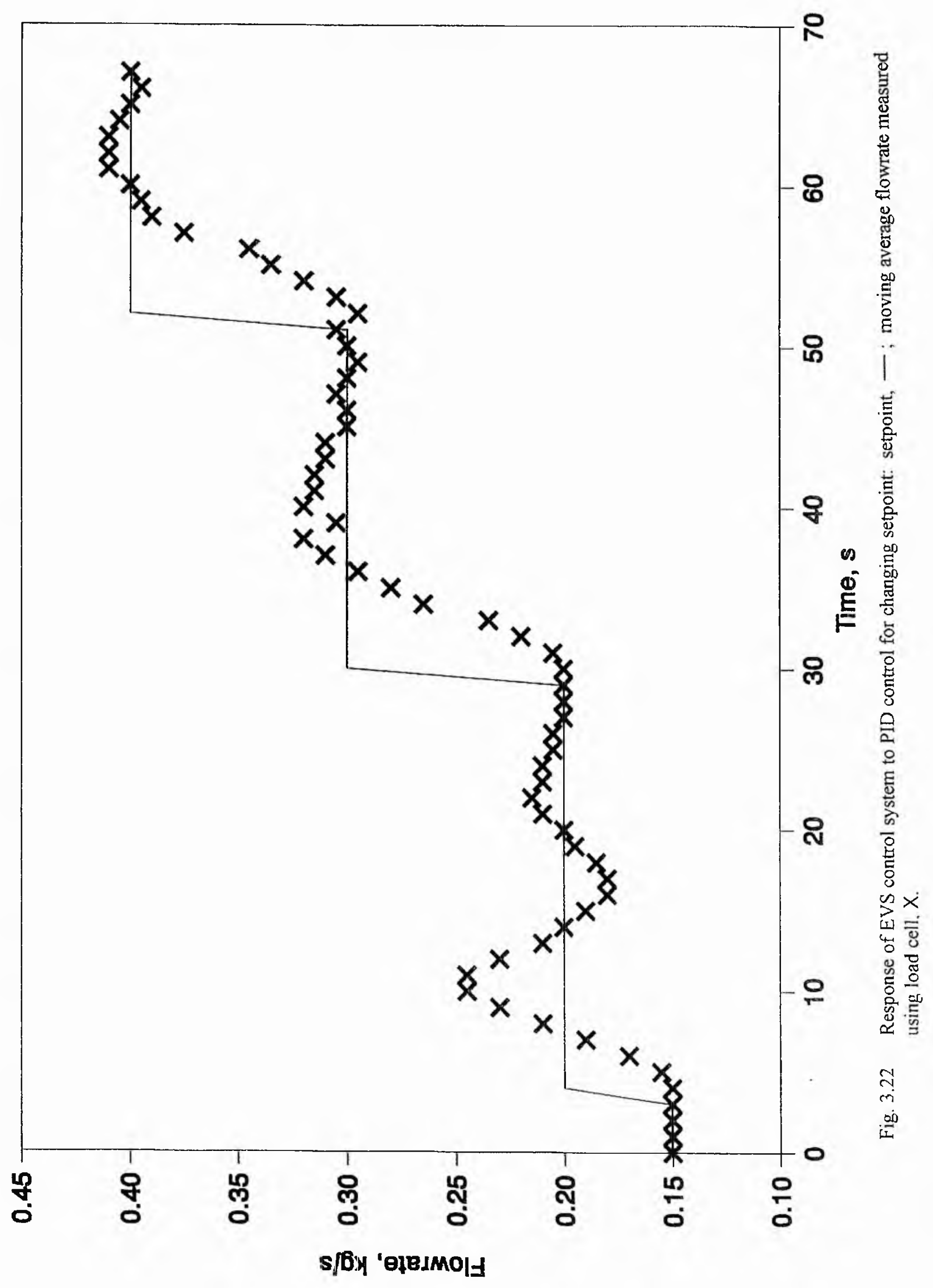




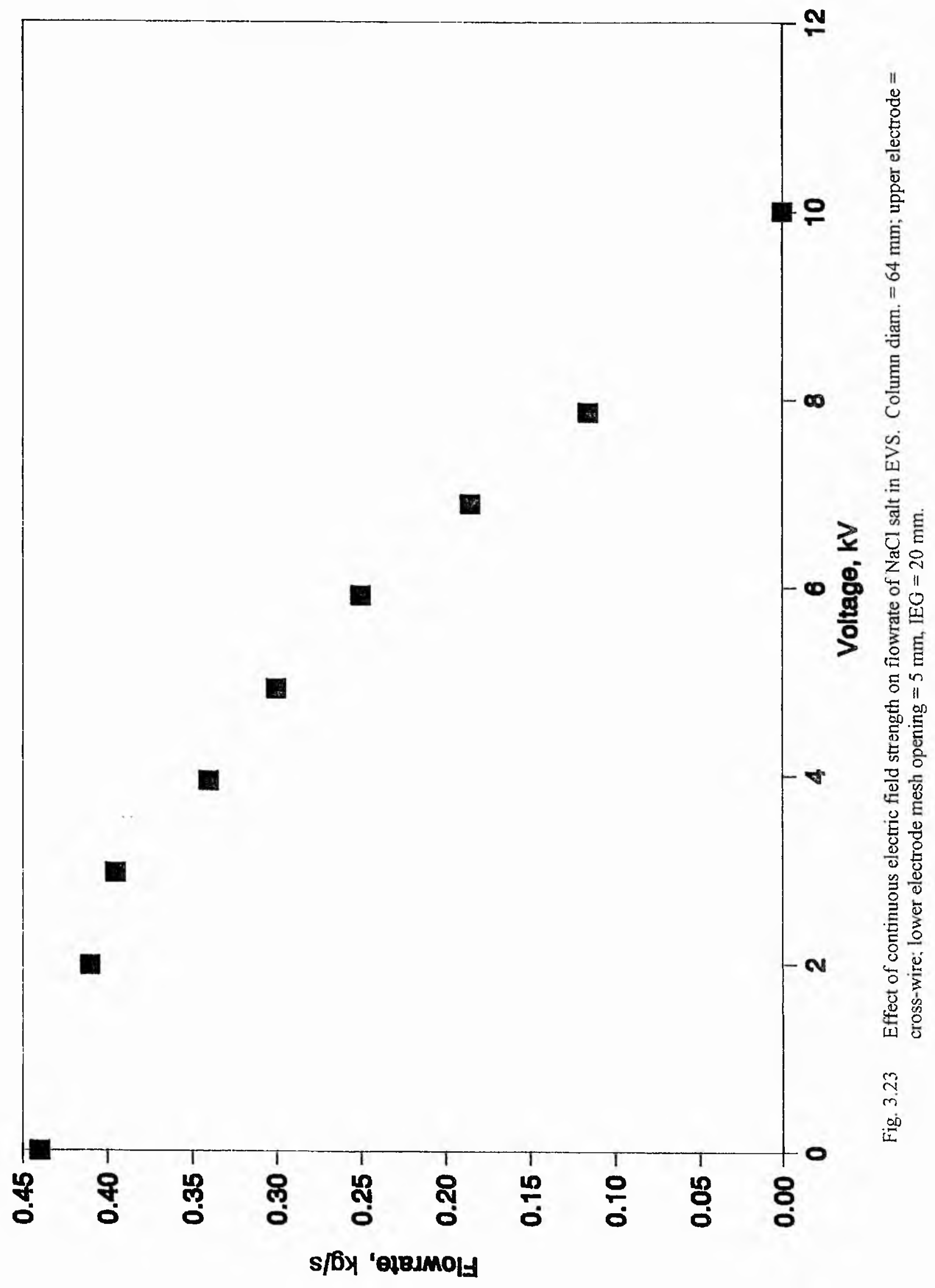



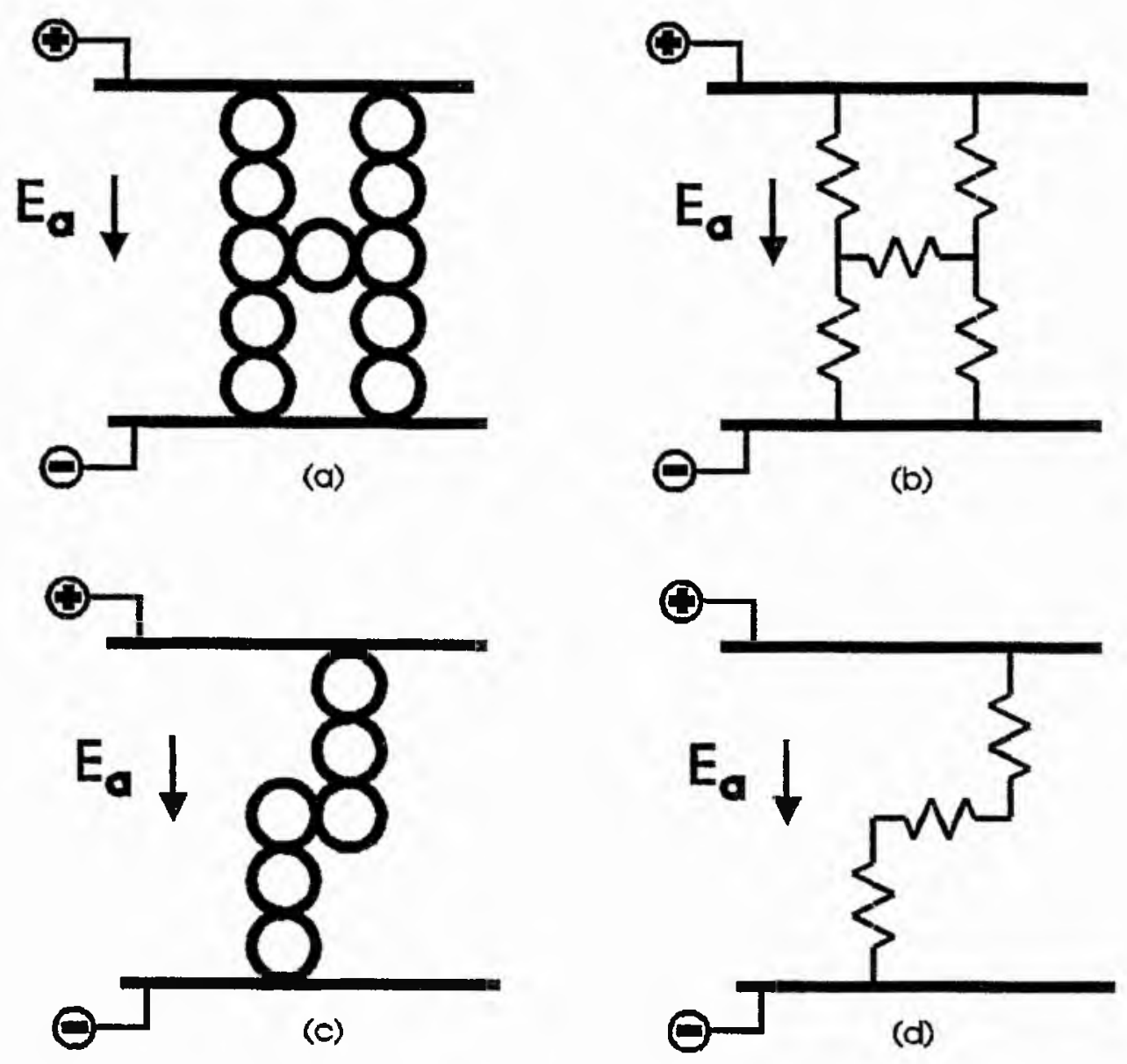

Fig. 4.1 Representation of current flow through particle assemblies. 


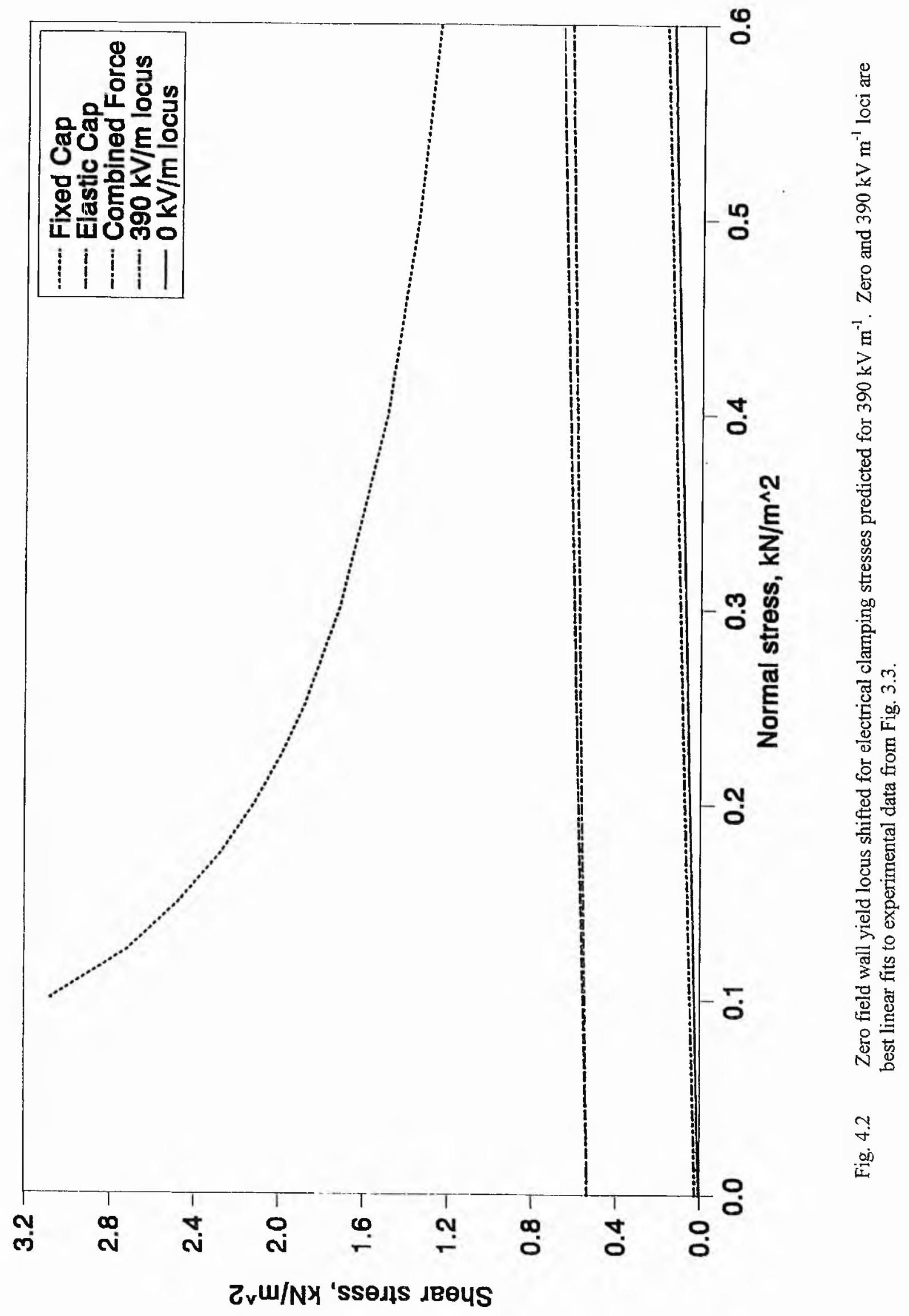


(a) Electrical stresses

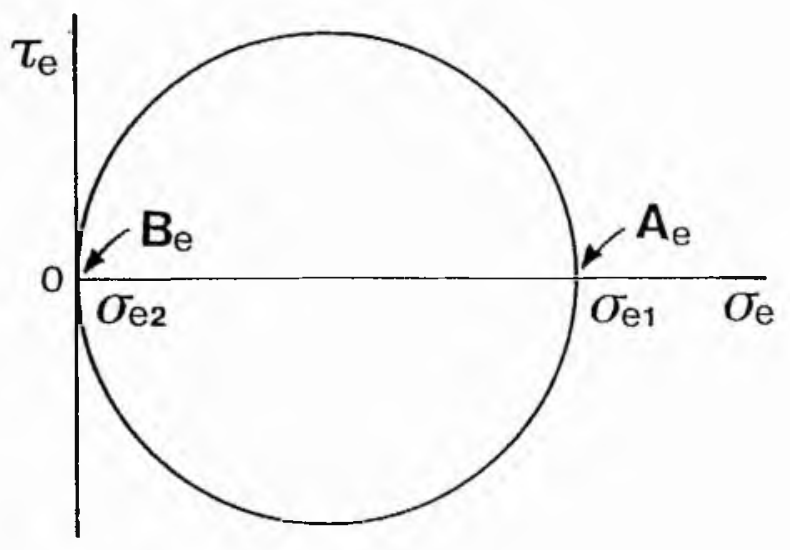

(b) Mechanical stresses

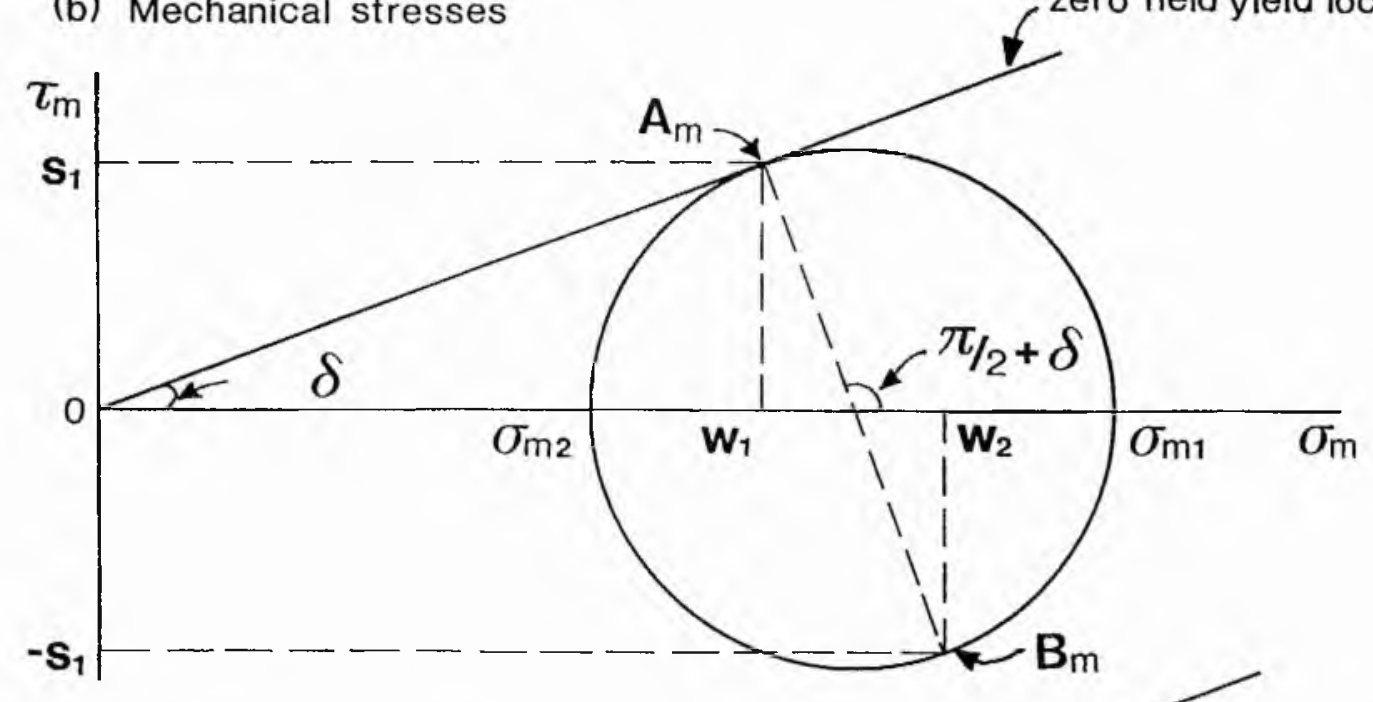

(c) Combined stresses

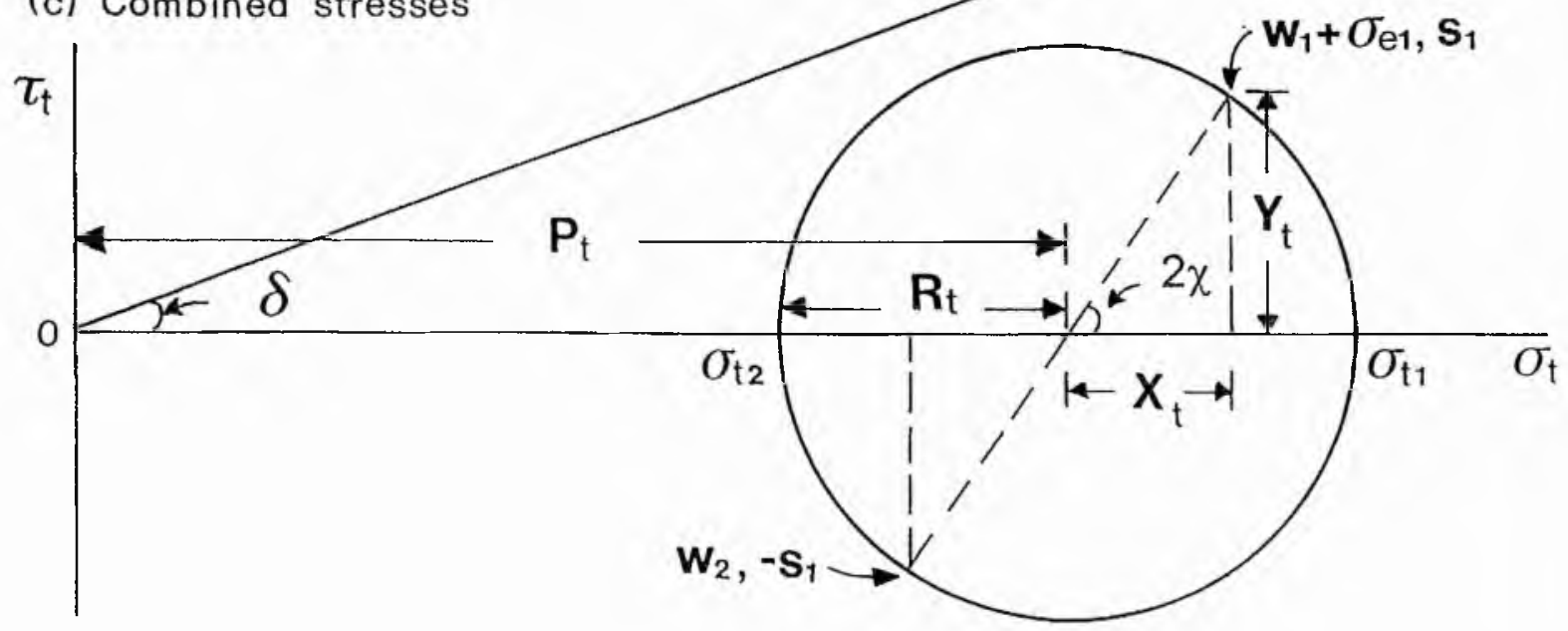

Fig. 4.3 Superimposition of electrical and mechanical stresses. 


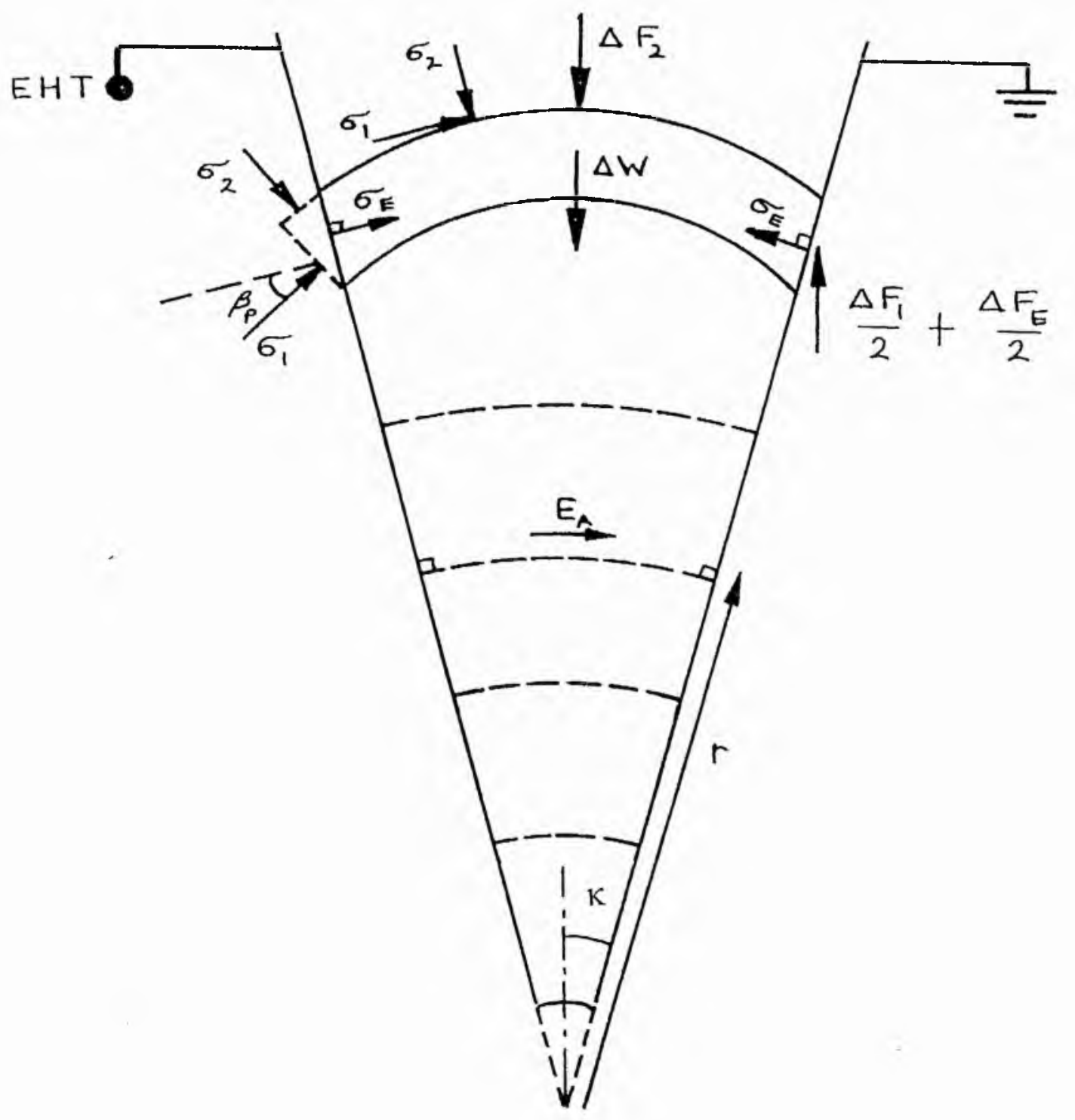

Fig. 4.4 Representation of mechanical and electrical stresses acting on arched element in wedge-shaped hopper. 


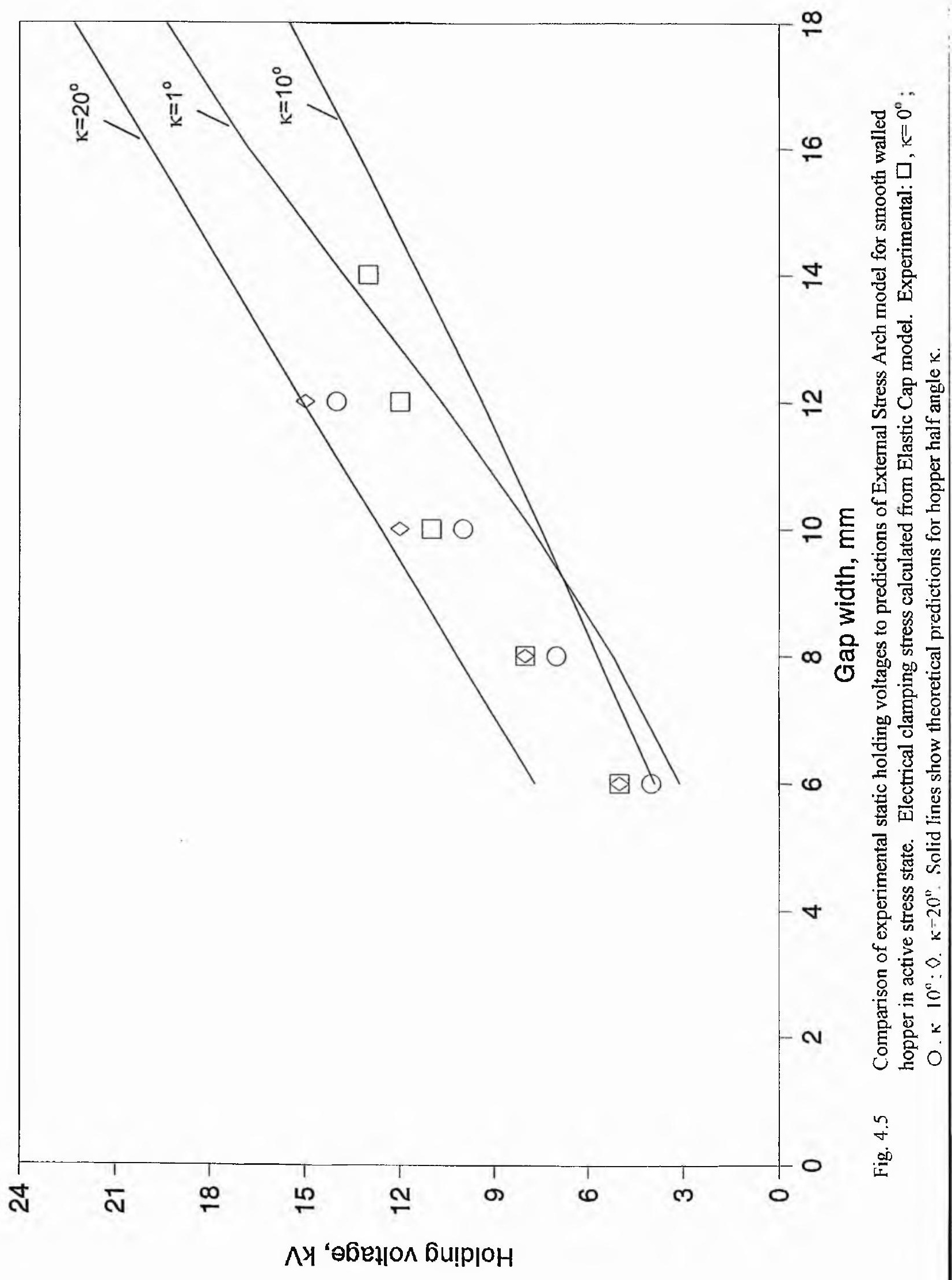




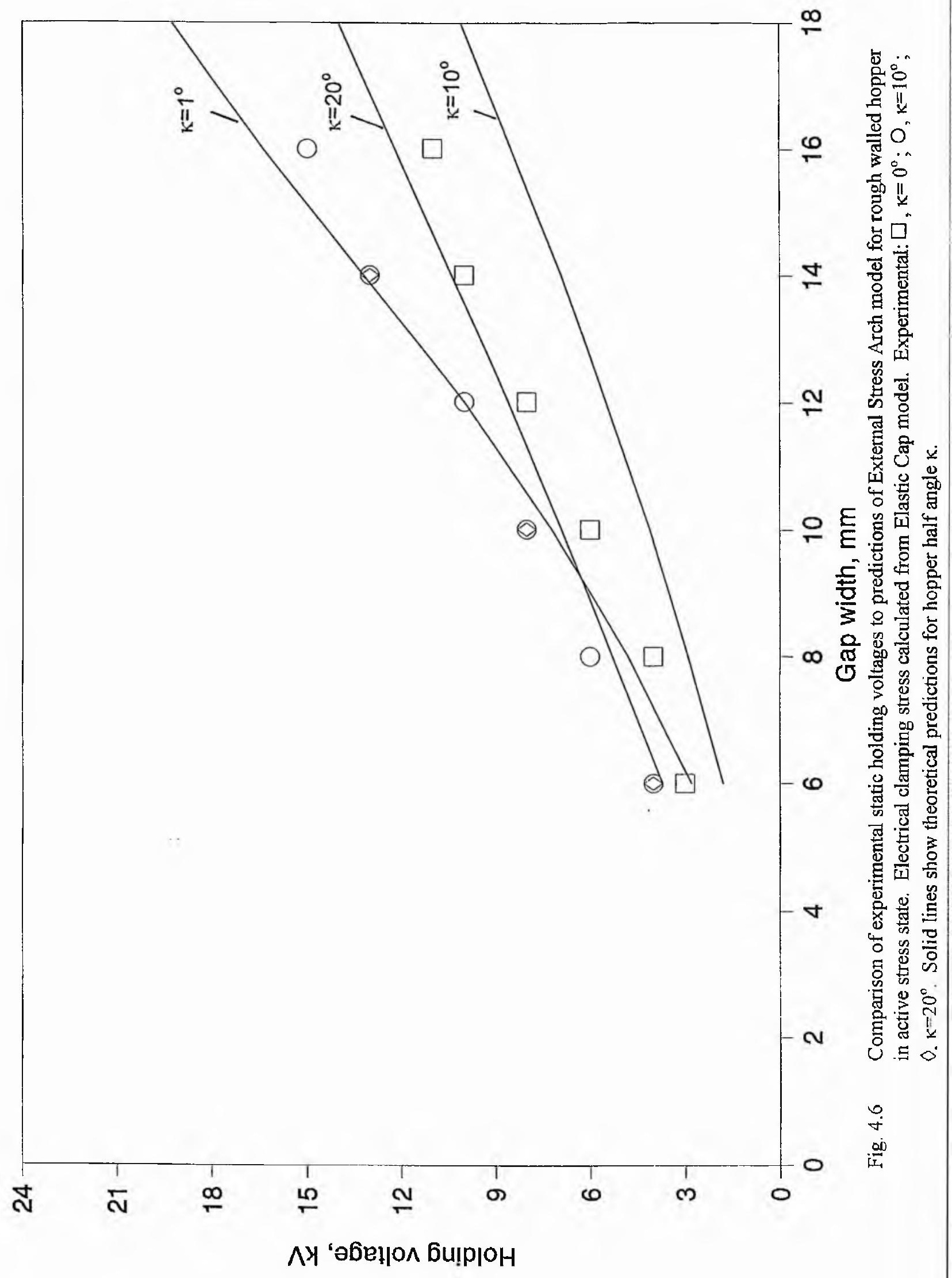




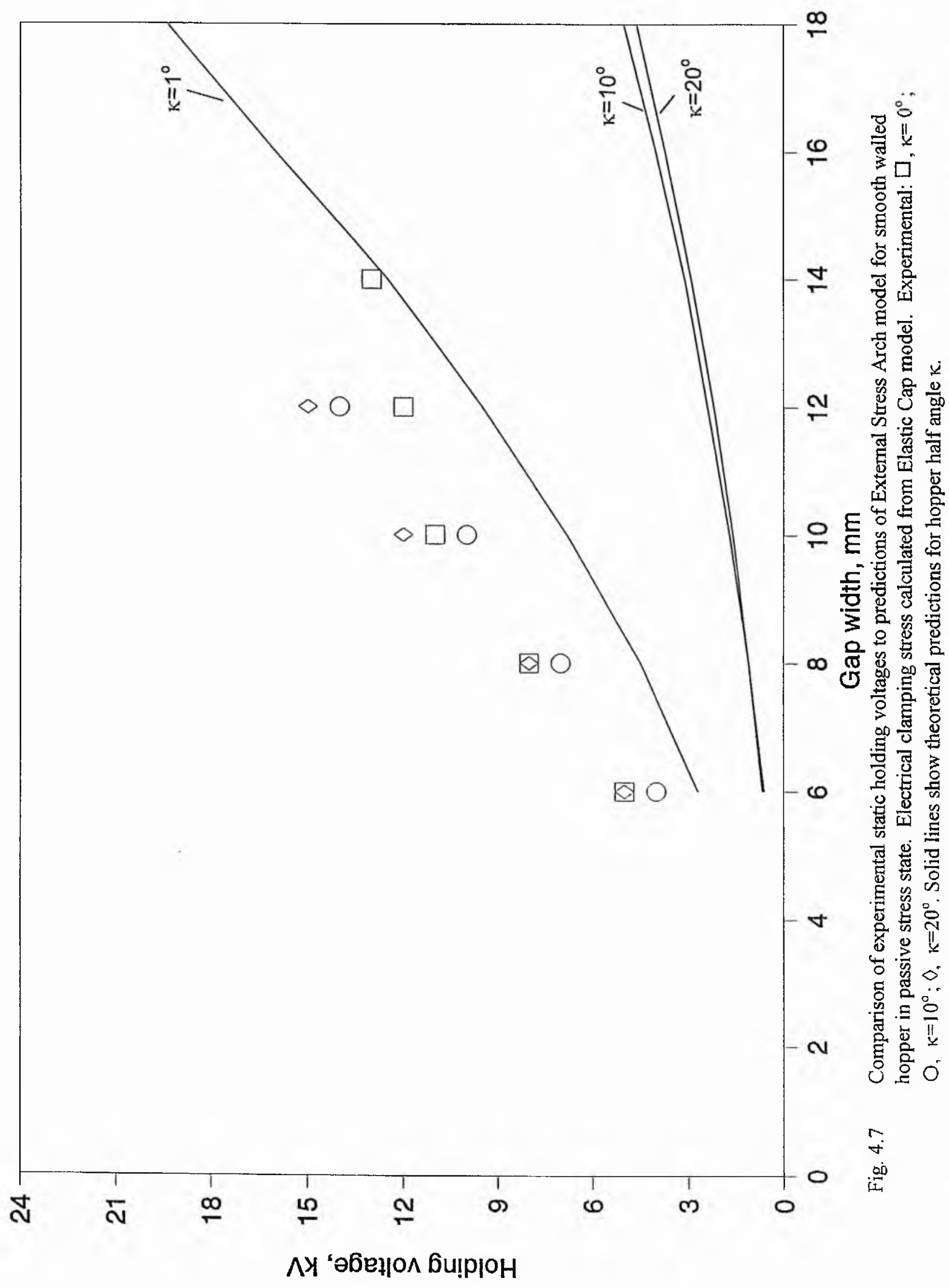




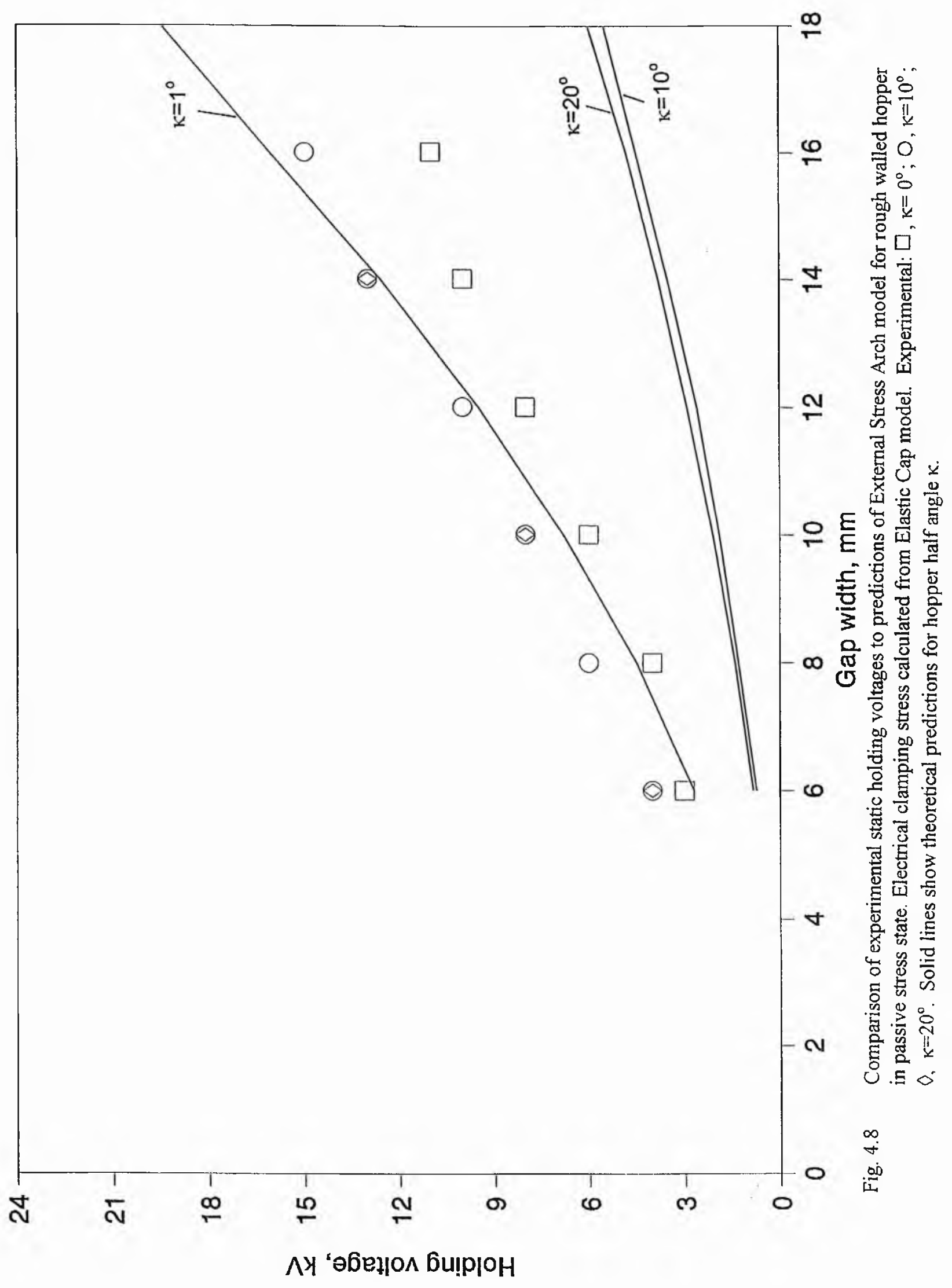




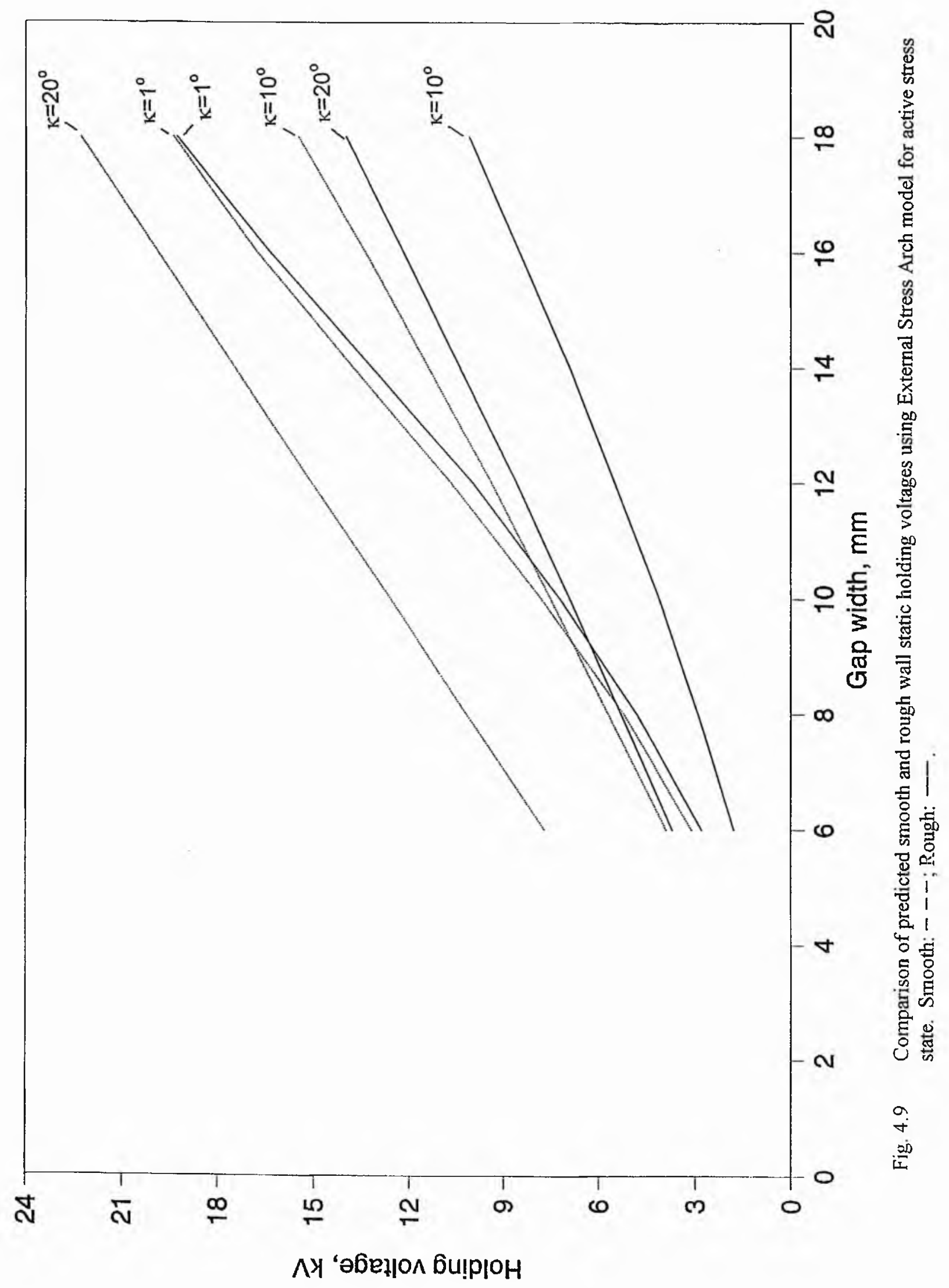




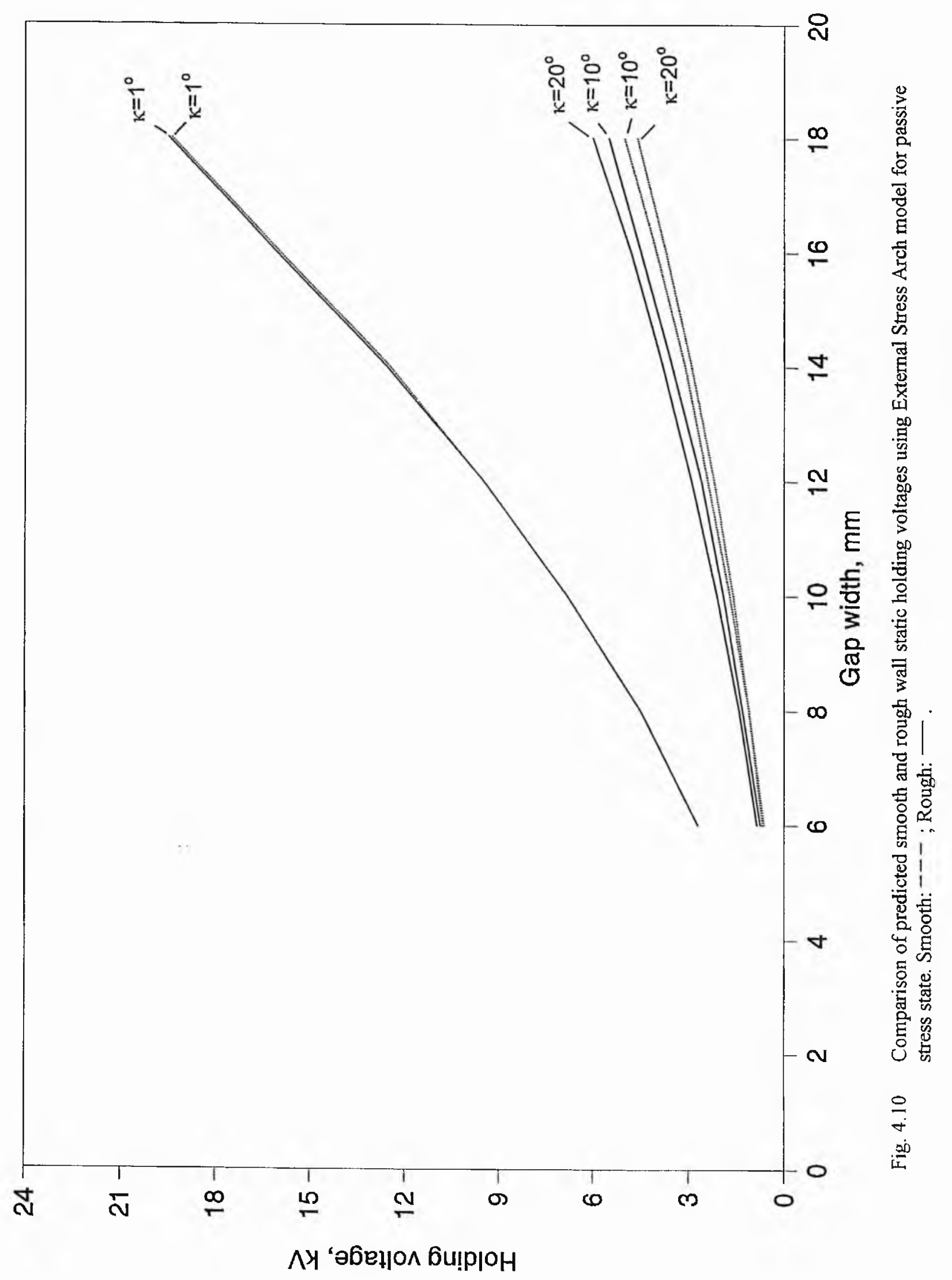




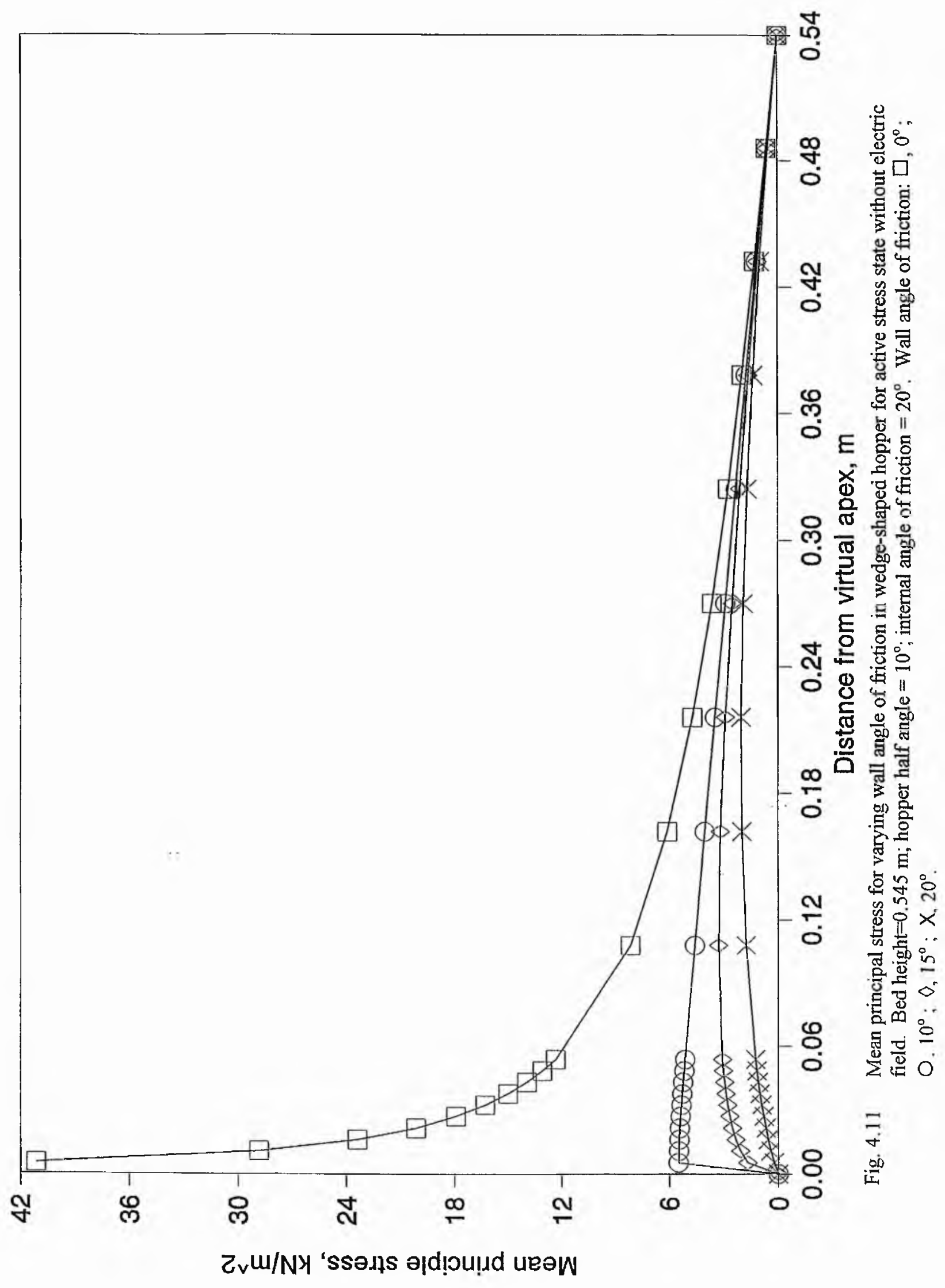




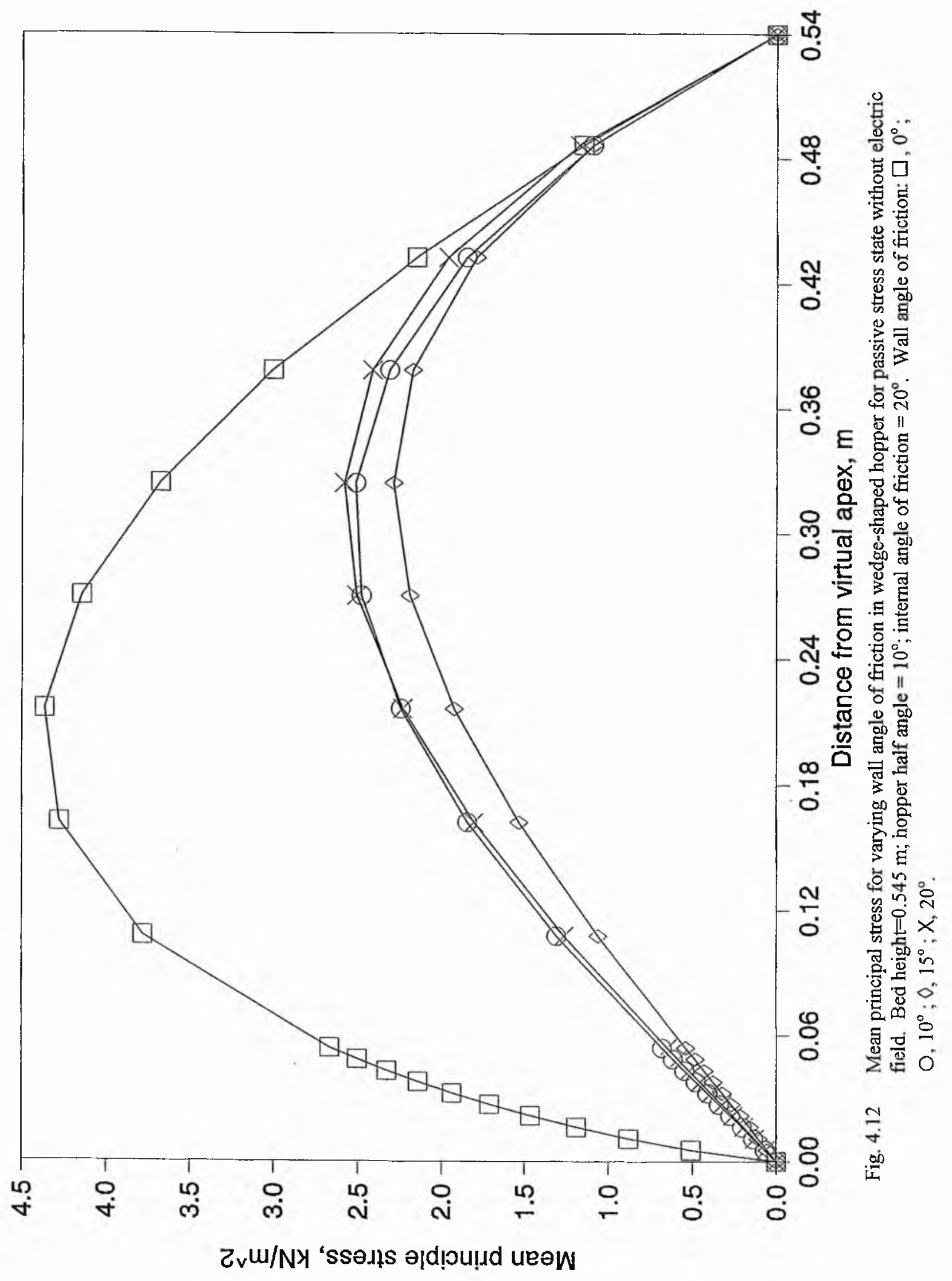



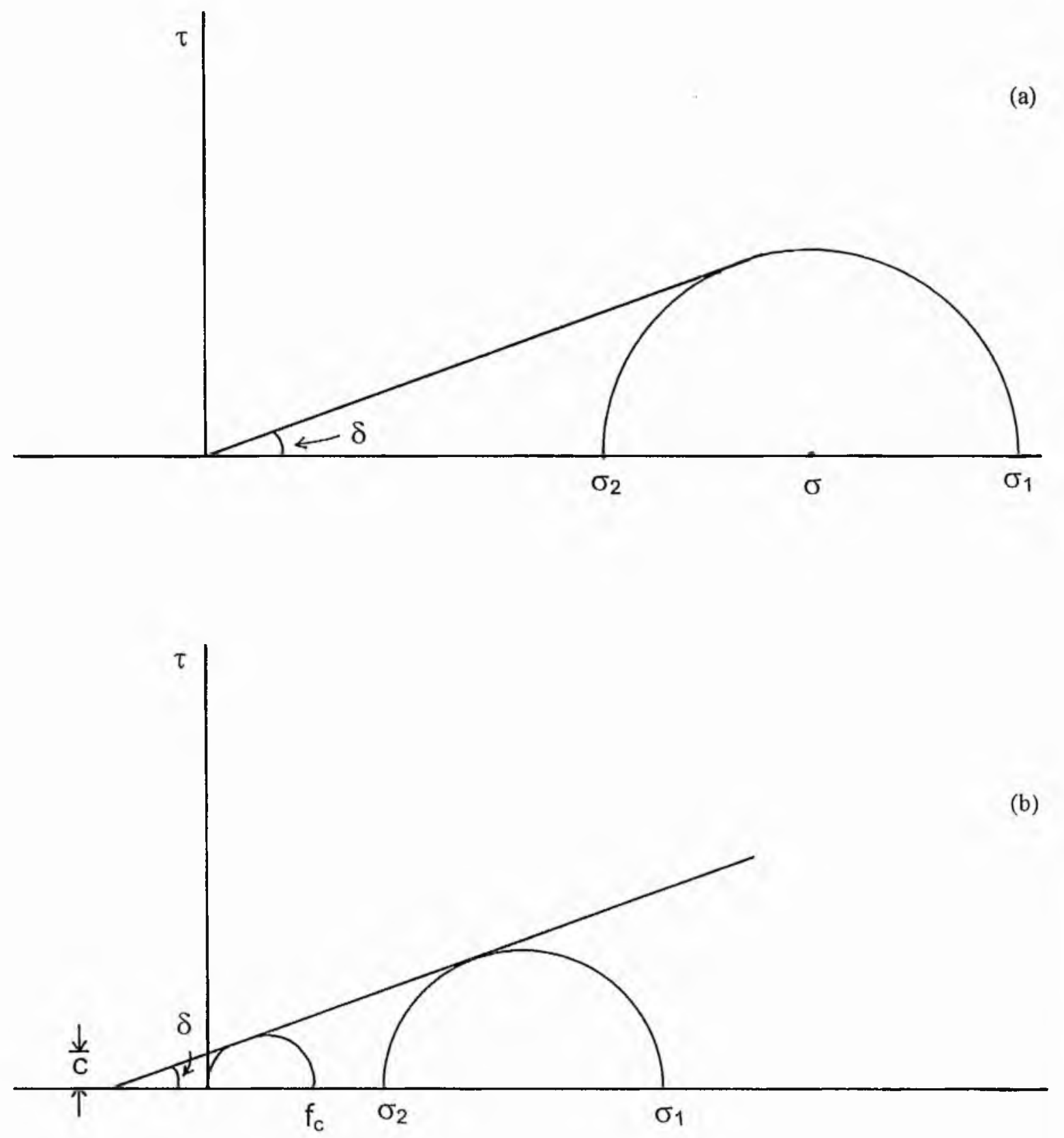

Fig. 4.13 Representation of yield loci and Mohr's stress circles for material at incipient yield. (a) Non-cohesive material in fully plastic stress state. (b) Cohesive material not in fully plastic stress state. 


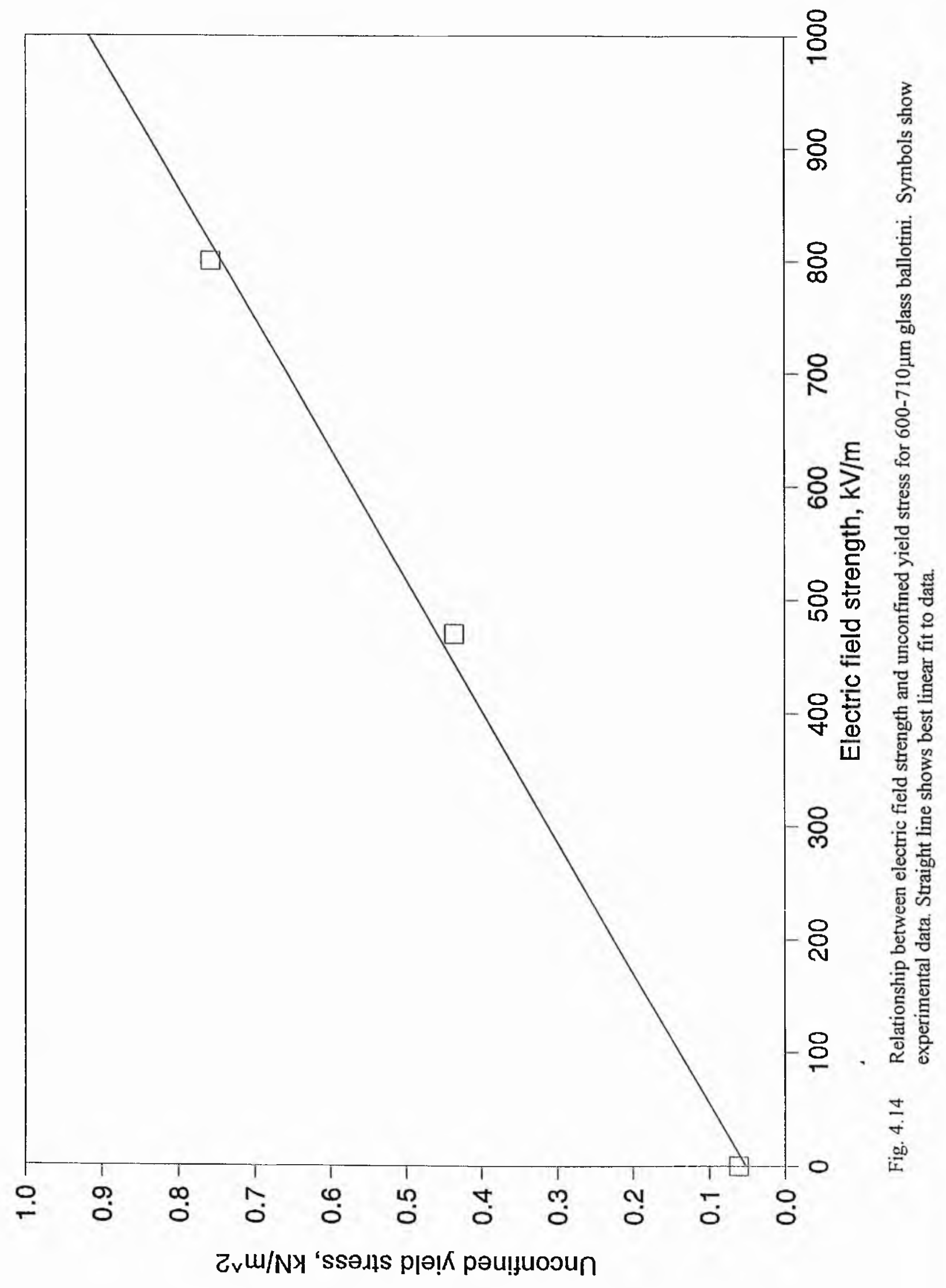




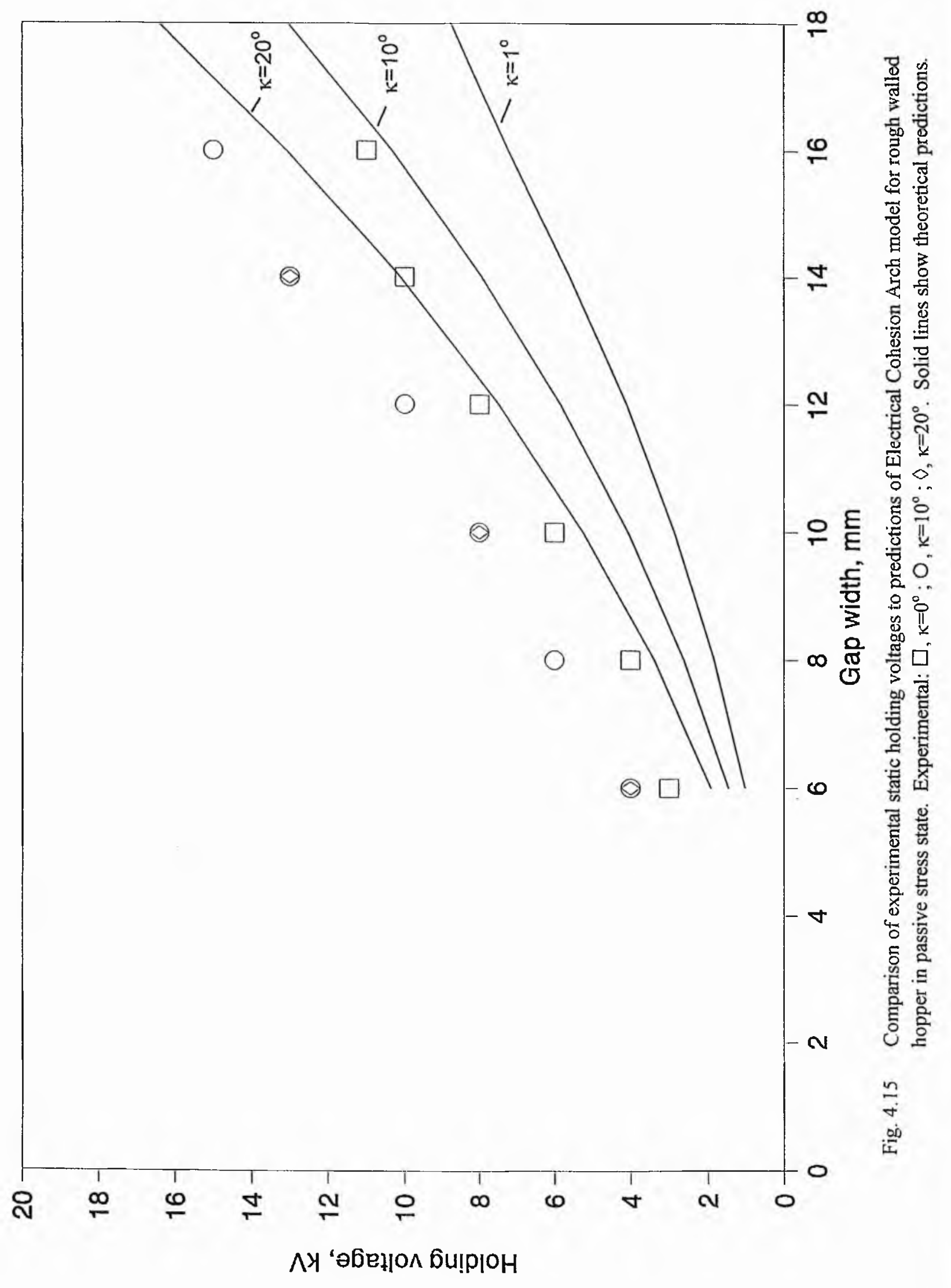




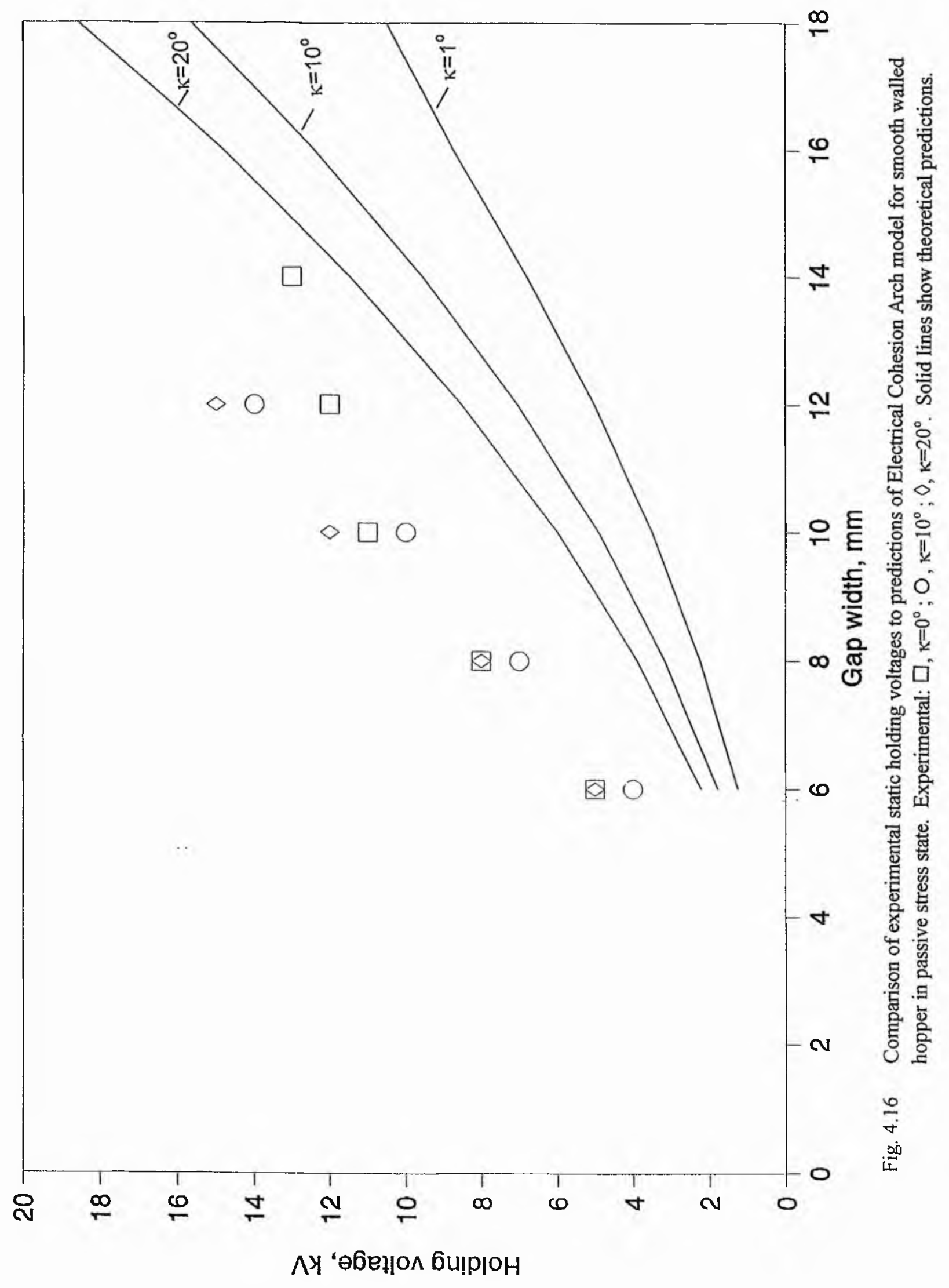




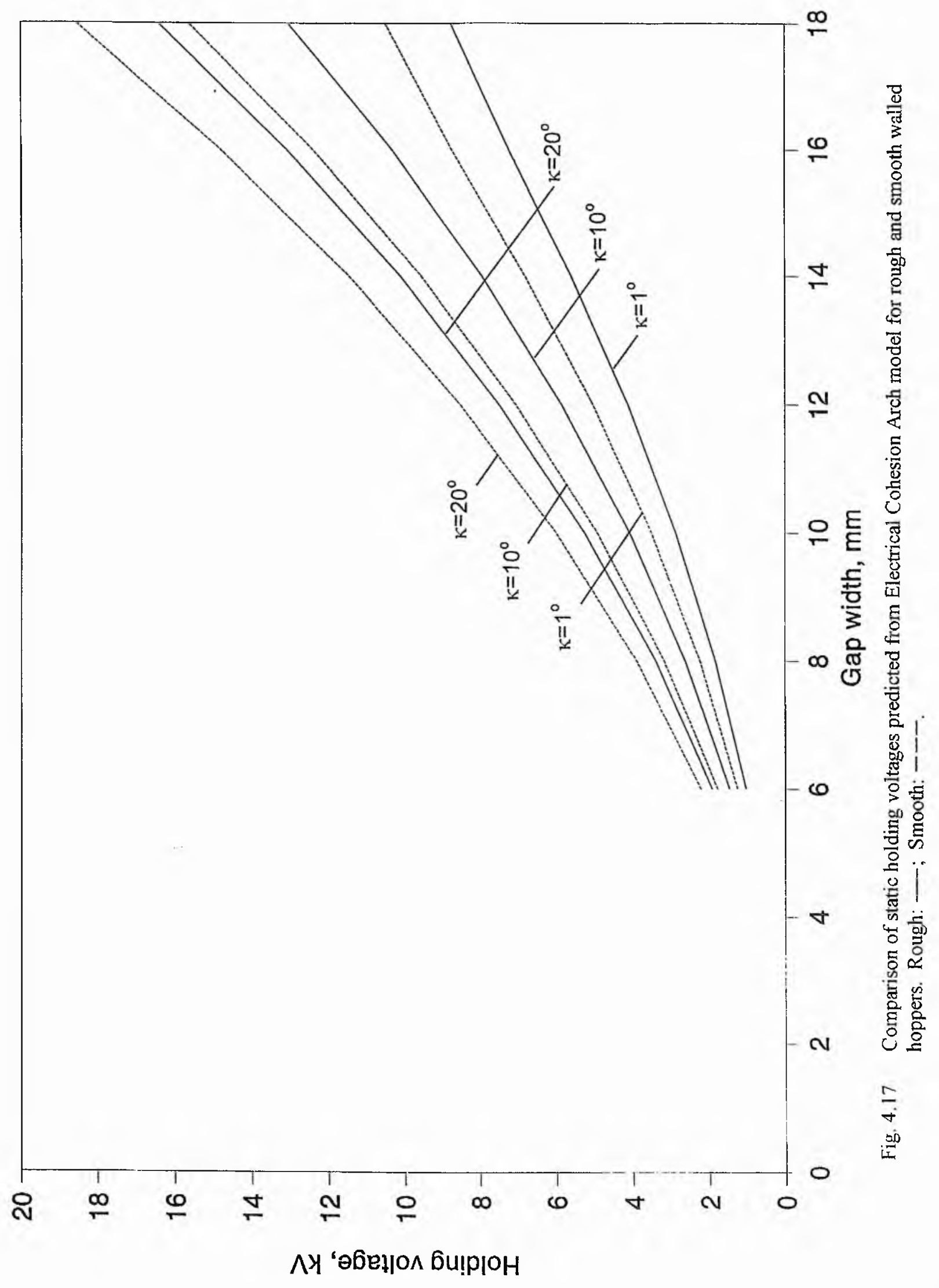




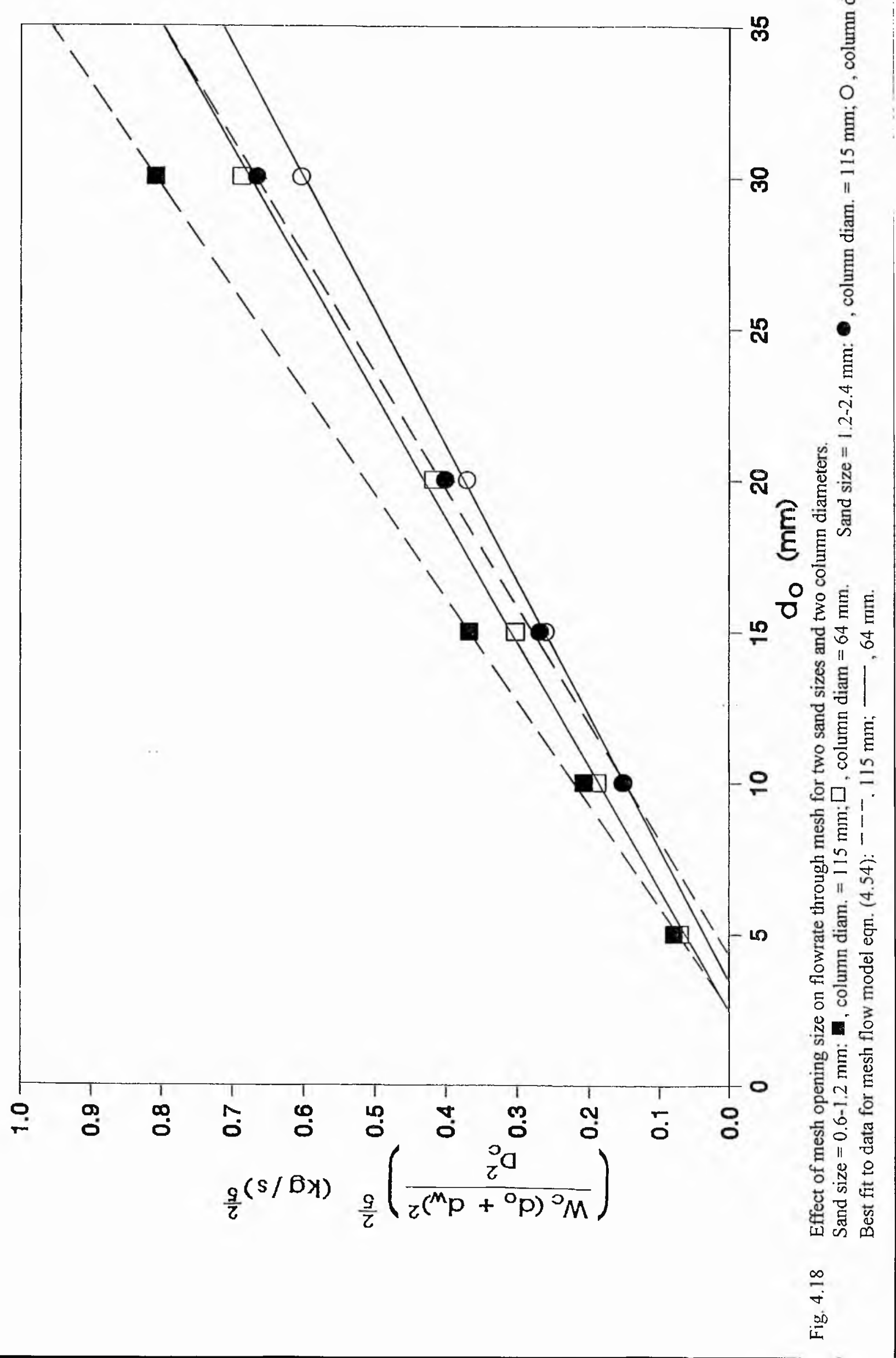




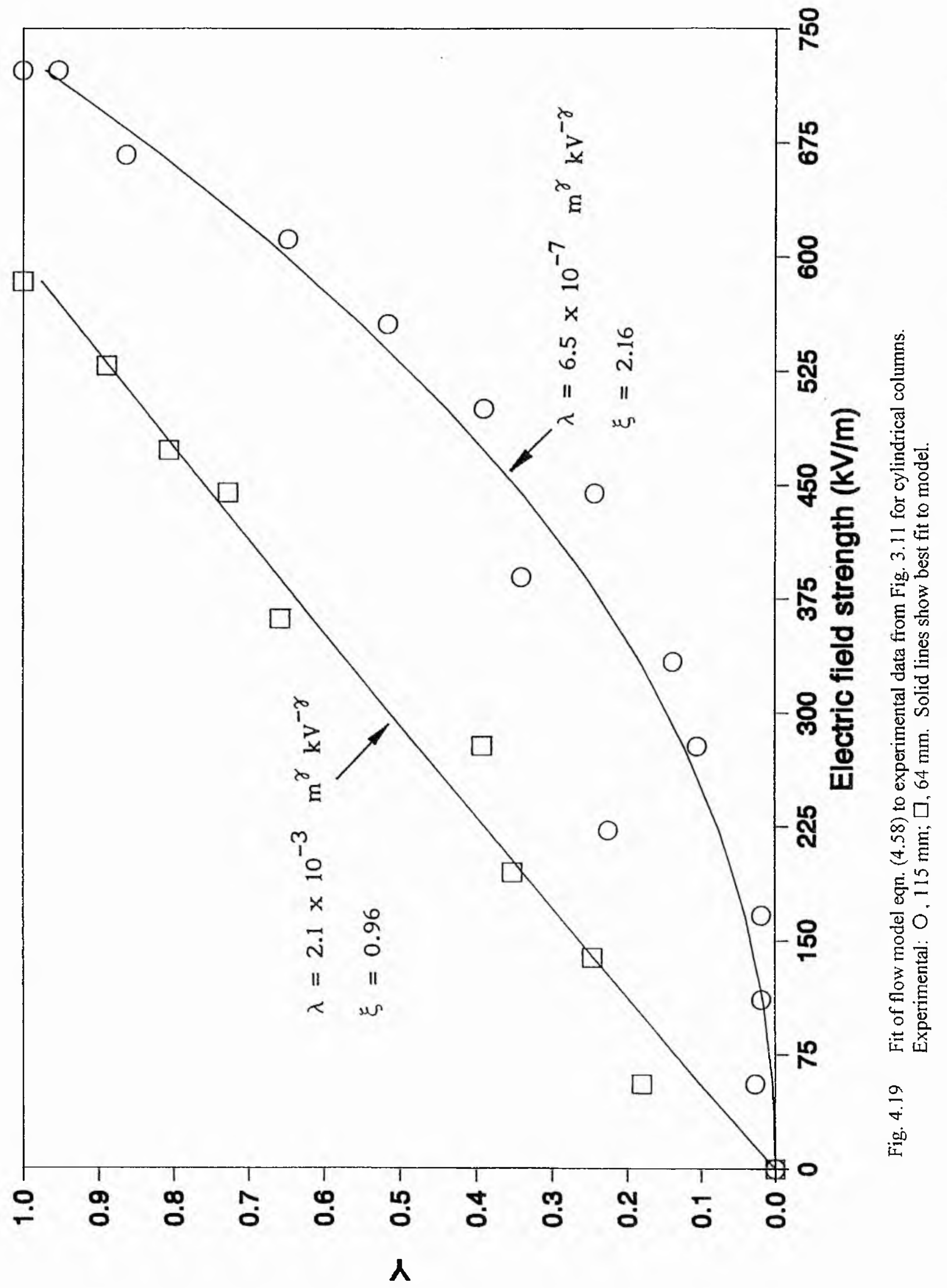




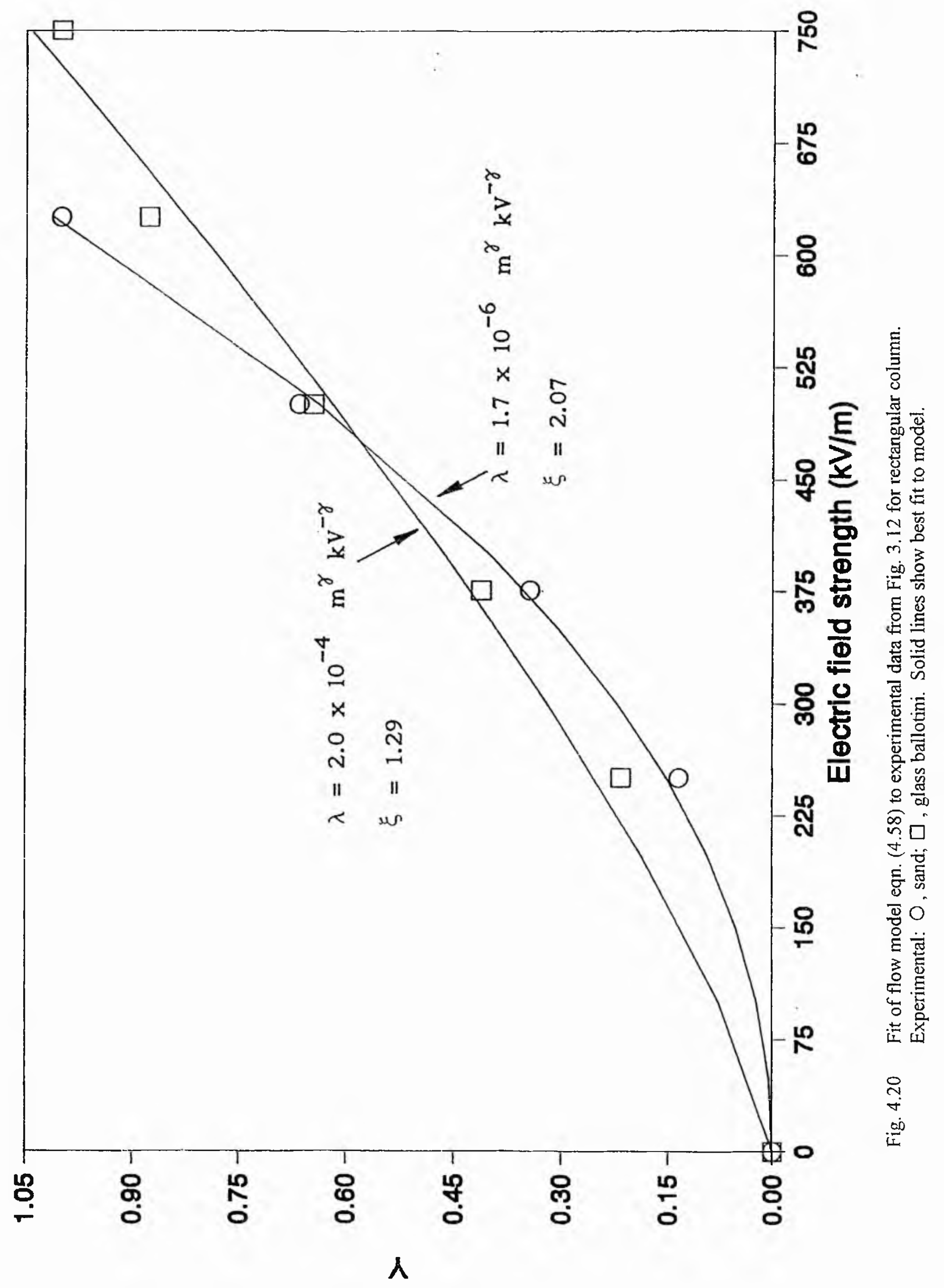




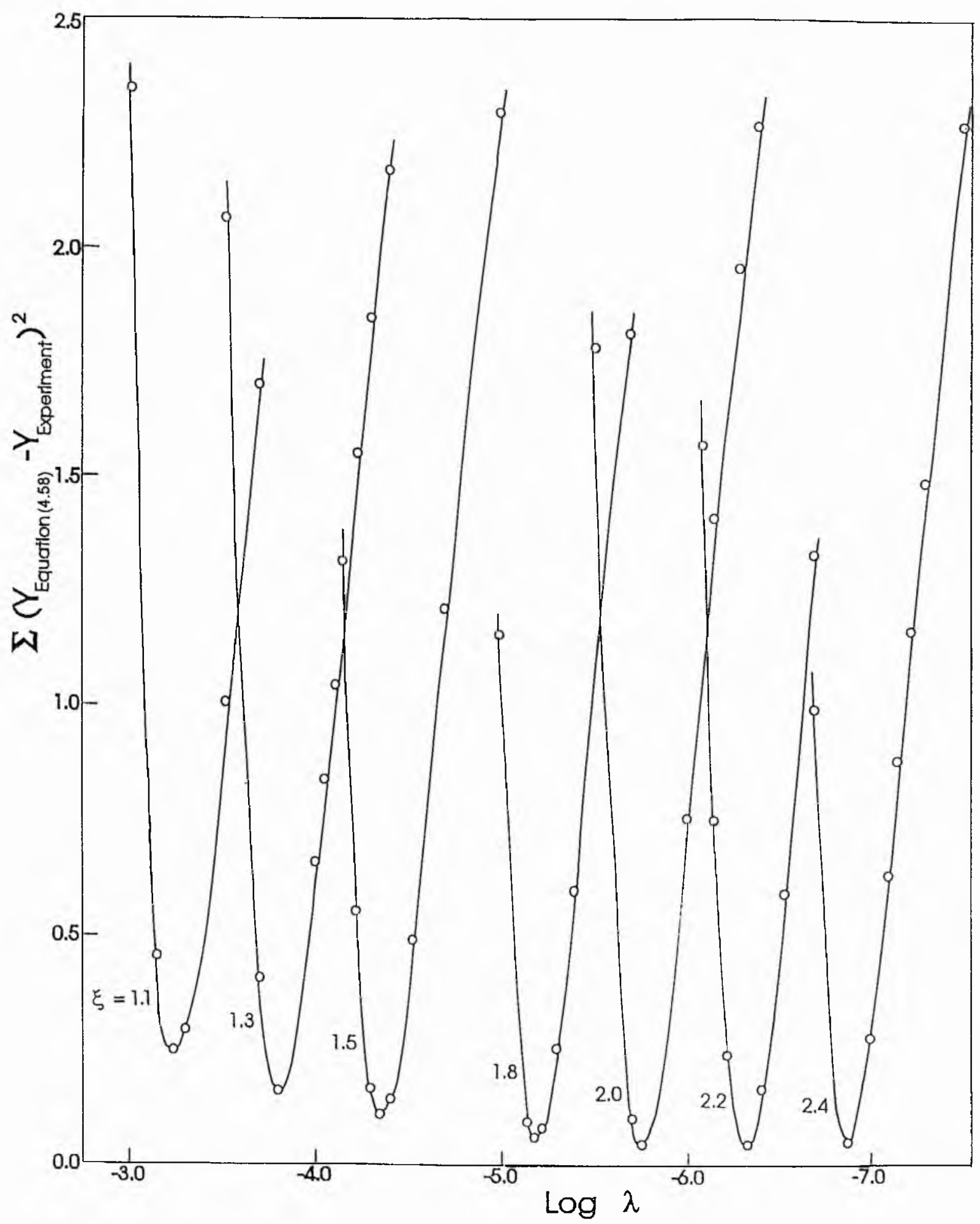

Fig. 4.21 Effect of varying $\lambda$ and $\xi$ on the sum of the squares of the error in $Y$ for flow model eqn. (4.58) for data from Fig. 3.11. 


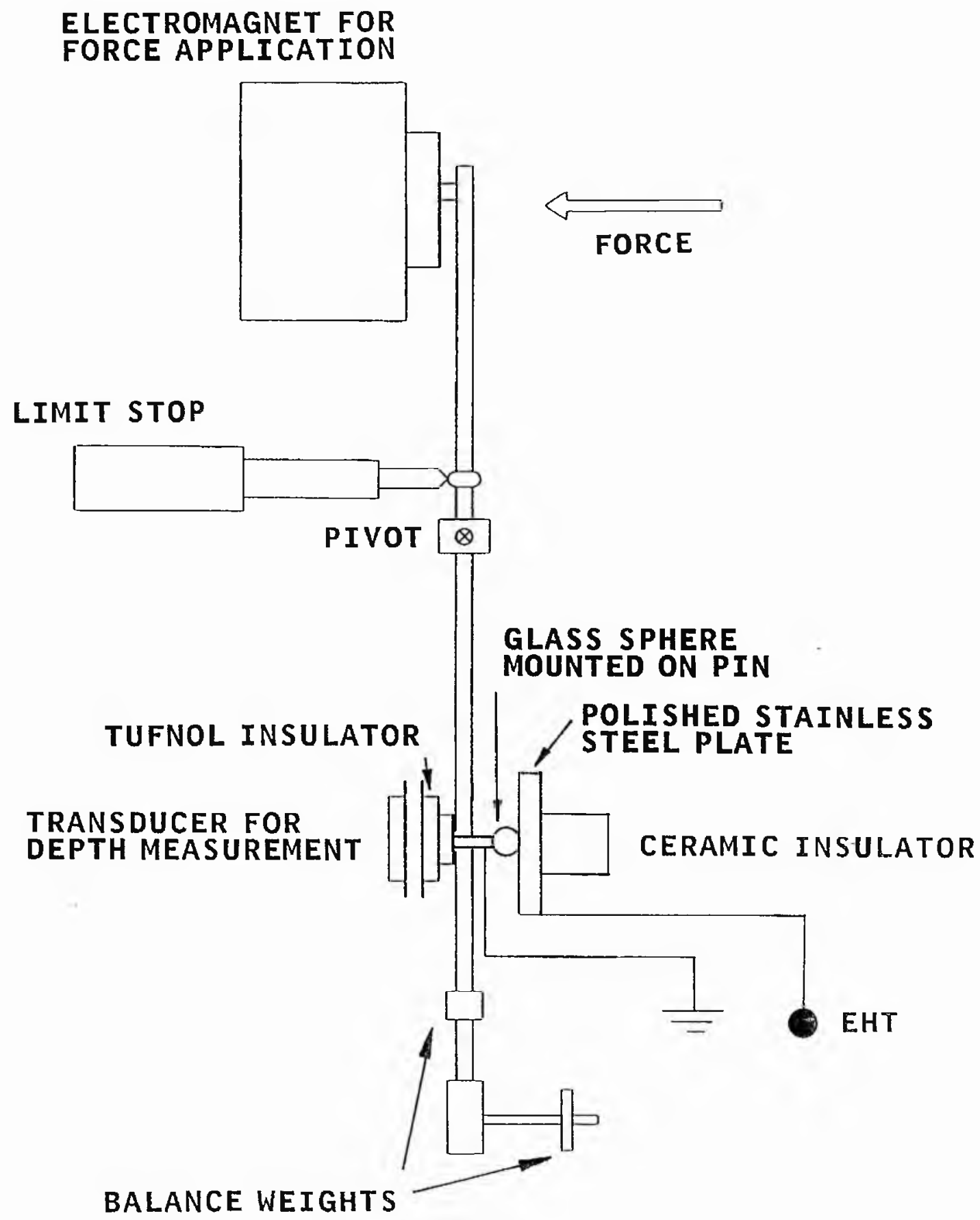

Fig. 5.1 Diagram of modified Nanotest device for measurement of electrical clamping force. 

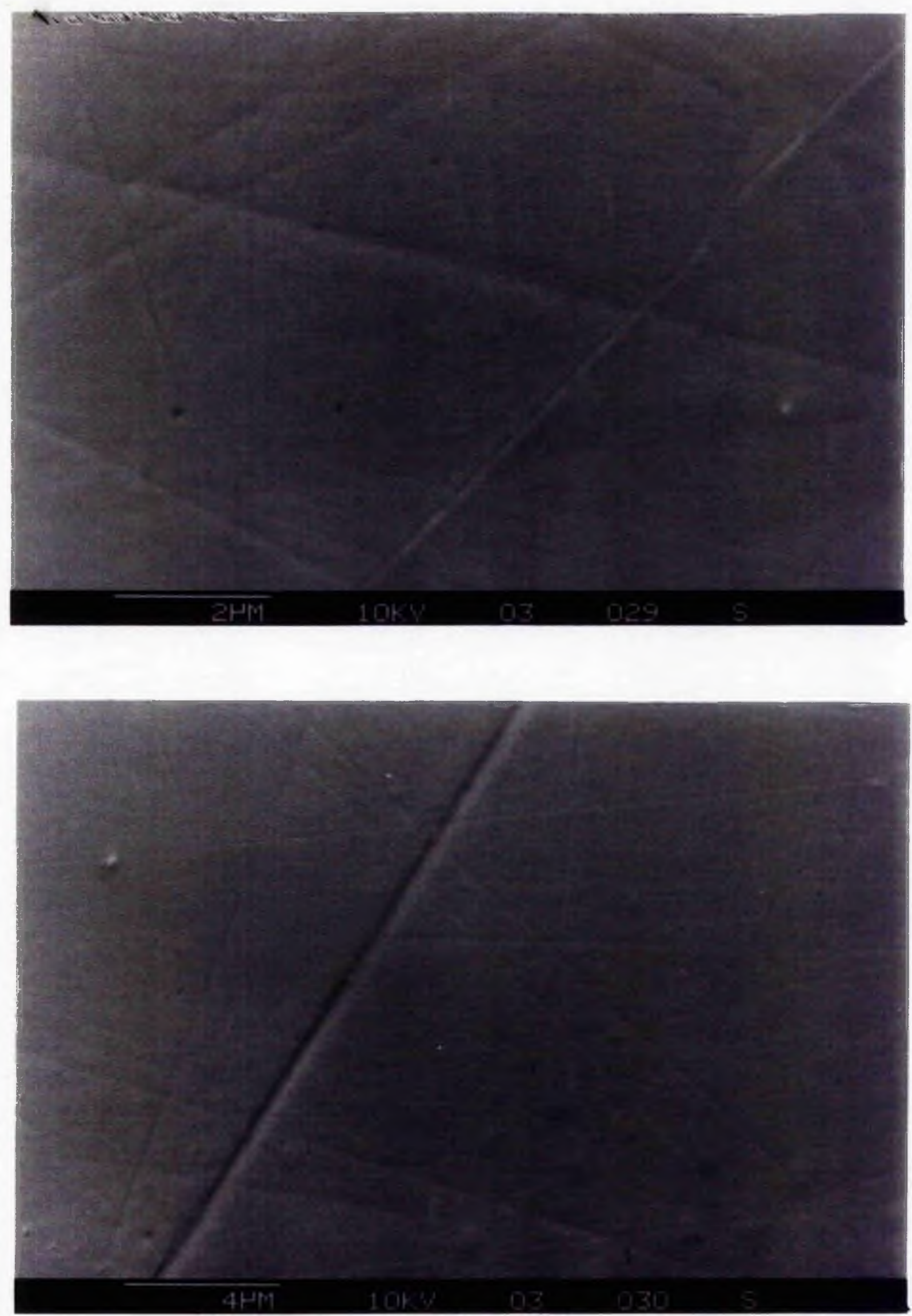

Fig. 5.2 Scanning electron micrographs of surface of polished stainless steel plate. 


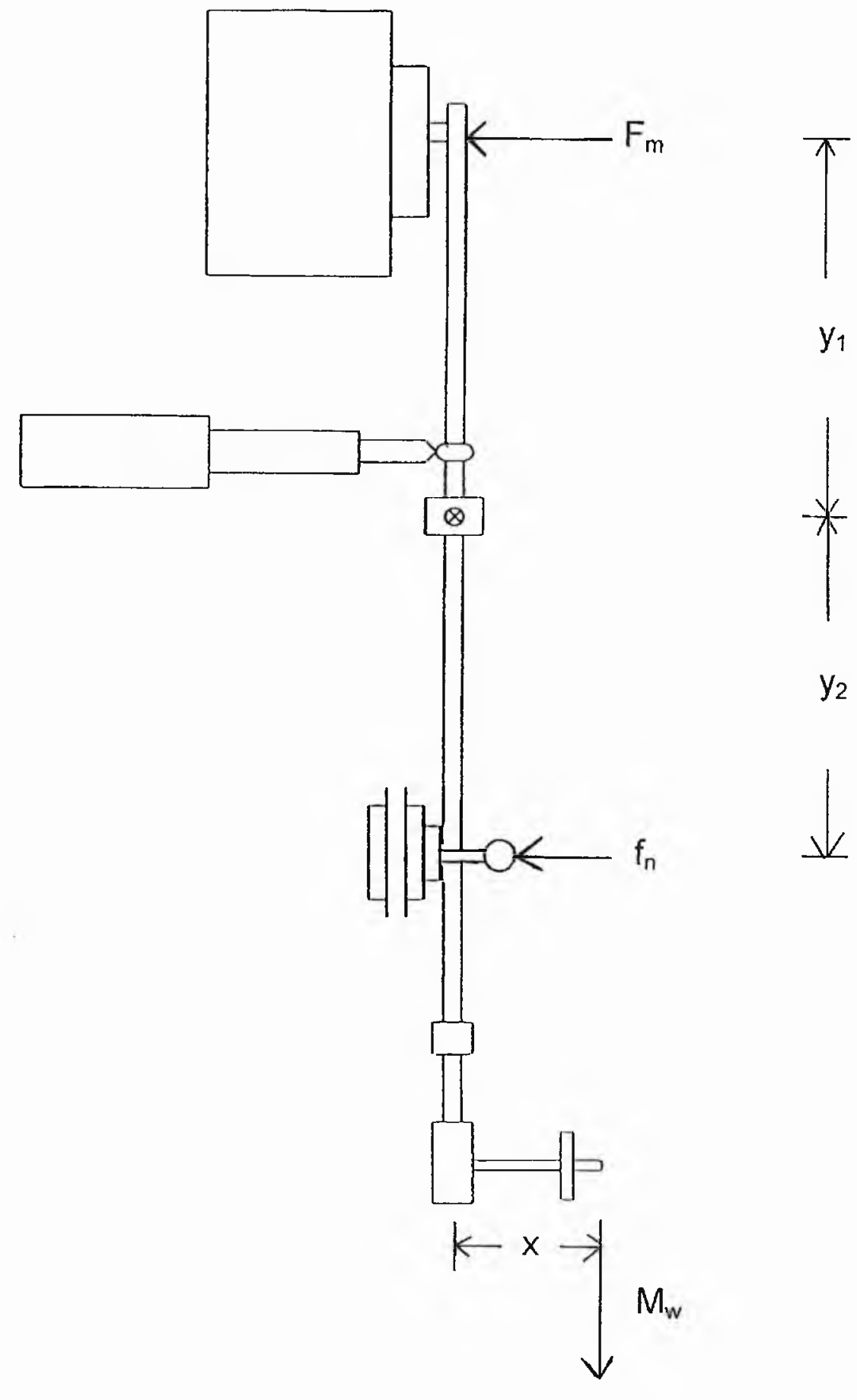

Fig. 5.3 Diagram of Nanotest pendulum showing dimensions used to calculate Load Calibration Factor in eqn. (5.4). 


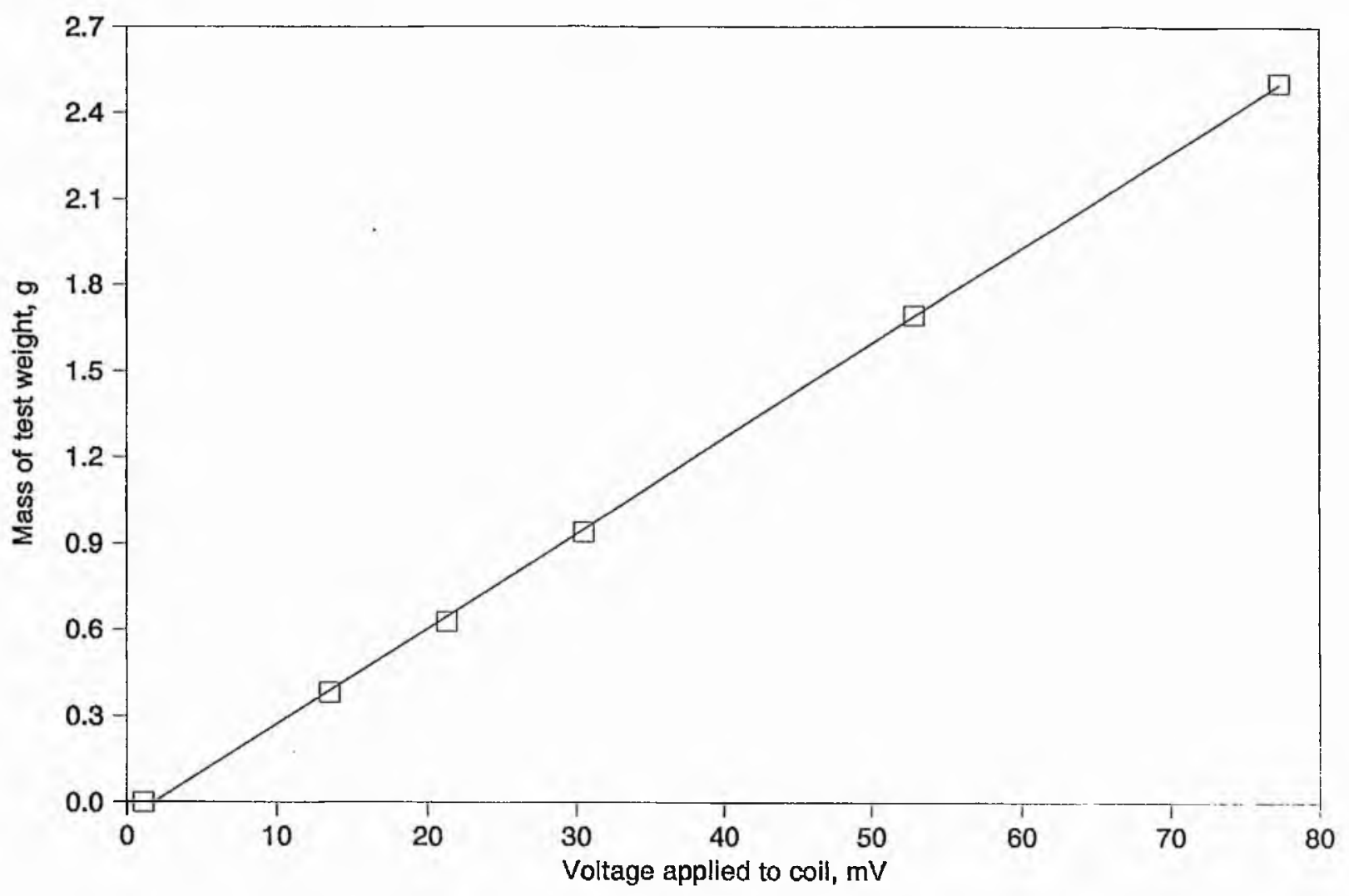

Fig. 5.4 Typical calibration curve for WV: Mass of dead weight versus voltage applied to coil to hold pendulum in equilibrium position. Symbols show experimental data. Line shows best linear fit to data. Slope of line $=3.31 \times 10^{-2} \mathrm{~g} \mathrm{mV}^{-1}$.

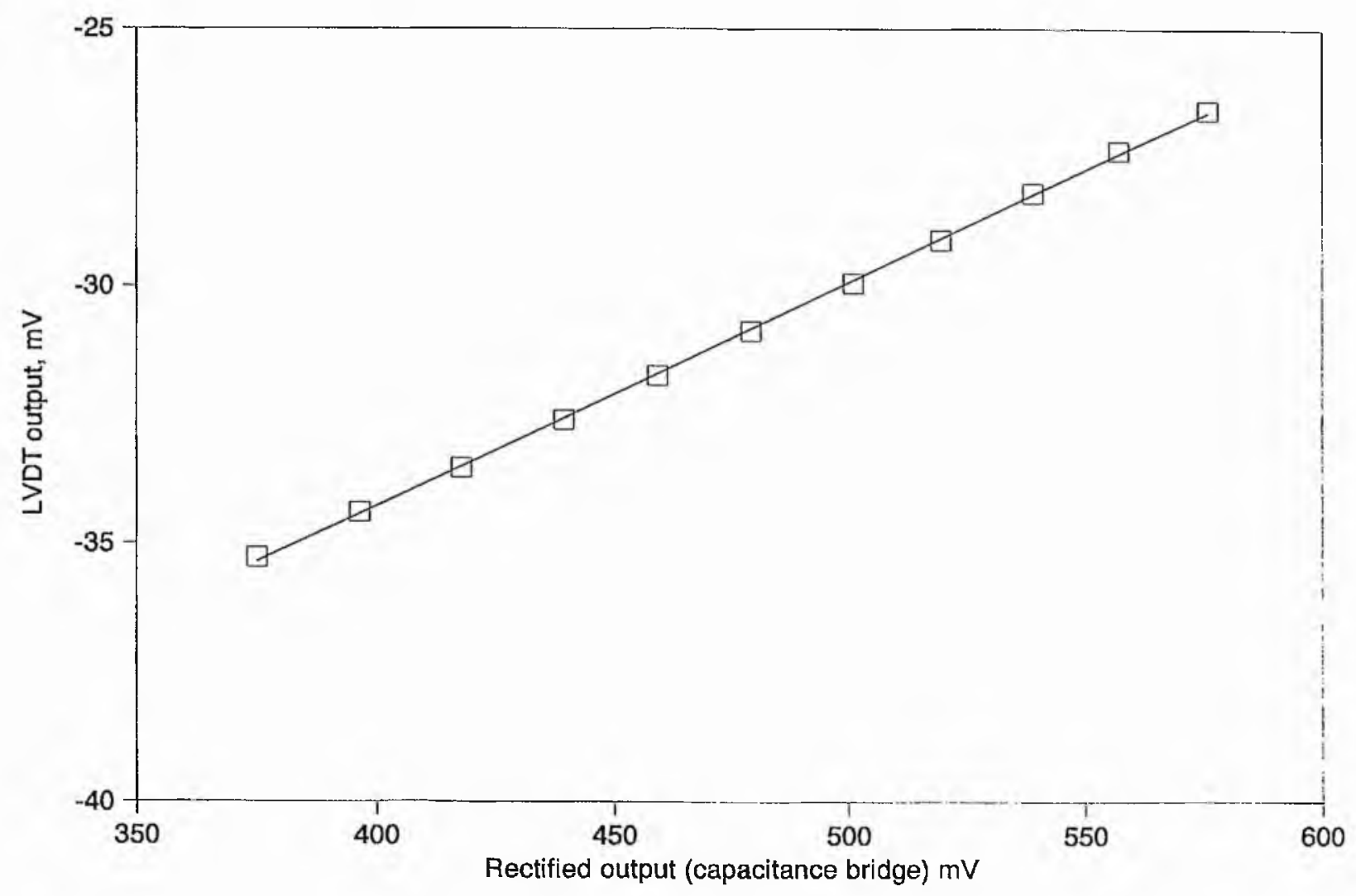

Fig. 5.5 Typical calibration curve for CR: LVDT output versus rectified output of capacitance bridge (negative LVDT output indicates compression). Symbols show experimental data. Line shows best linear fit to data. Slope of line $=4.34$ $x 10^{-2} \mathrm{mV} \mathrm{mV} \mathrm{m}^{-1}$. 


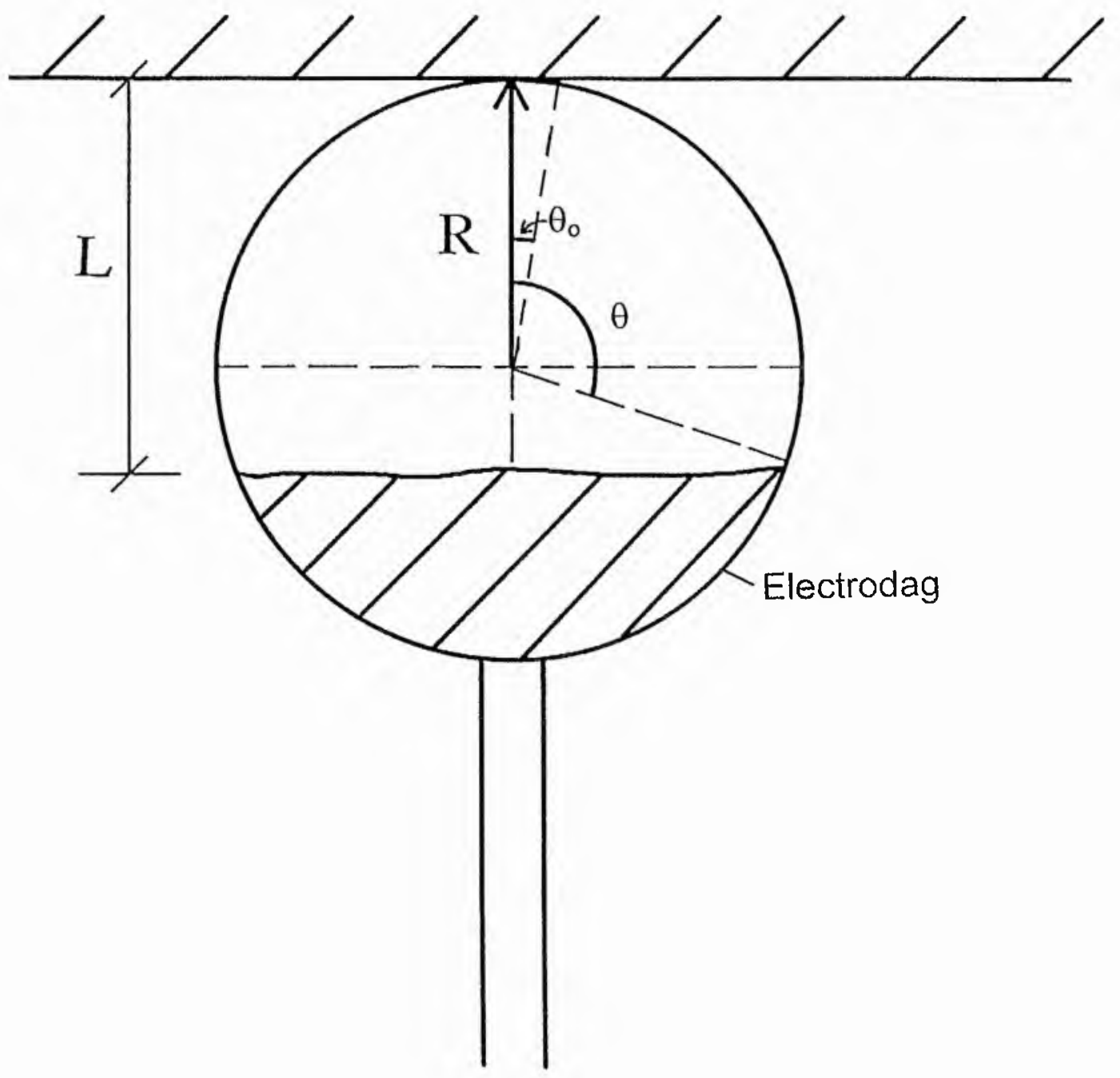

Fig. 5.6 Schematic diagram of mounted glass sphere. 

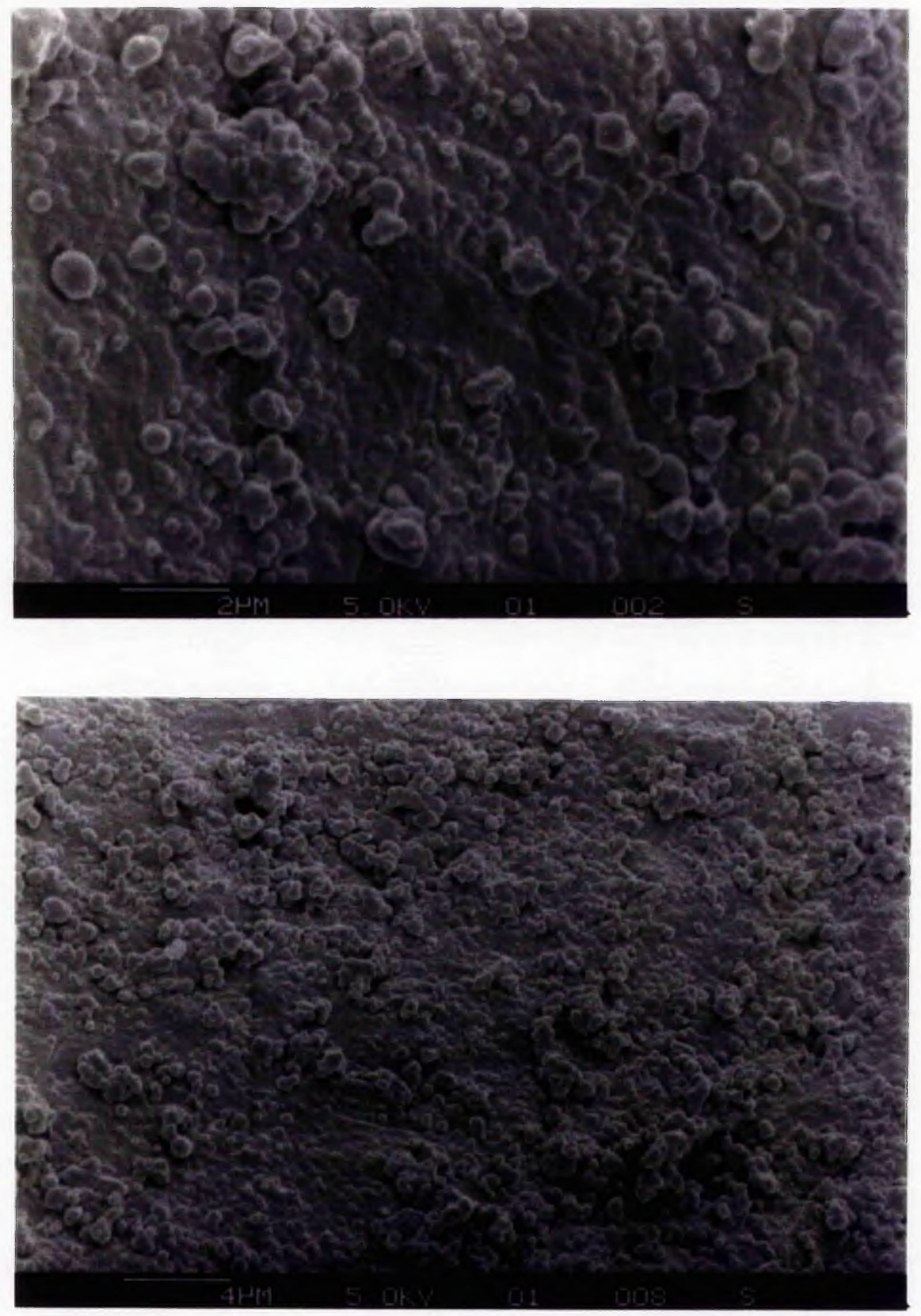

Fig. 5.7 Scanning electron micrographs of glass spheres coated with surfactant. 


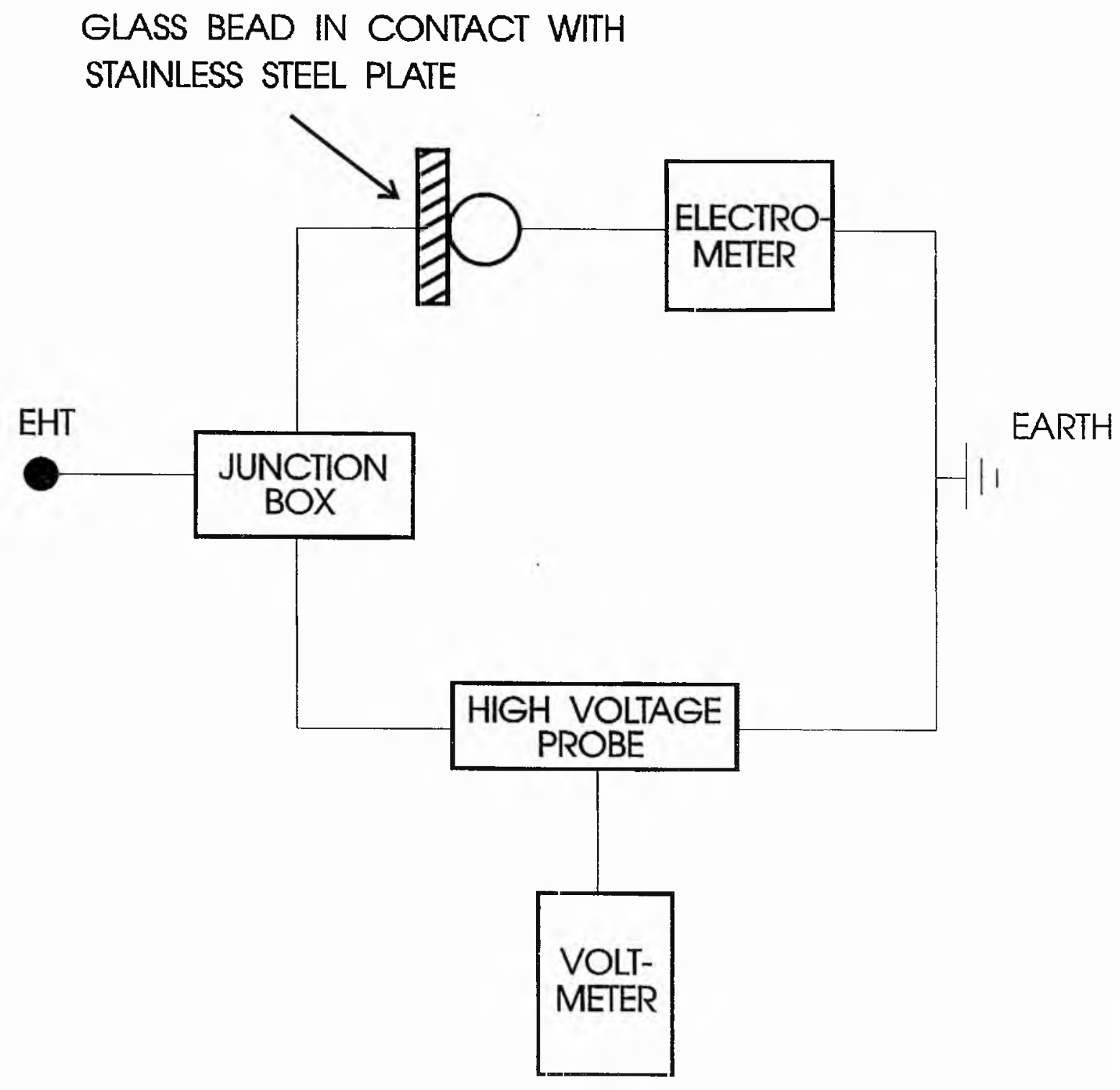

Fig. 5.8 Schematic diagram of experimental setup for electrical clamping force measurement. 
Sphere breaks contact with wall and pendulum swings away from wall

Tensile force ramp switched off after contact broken

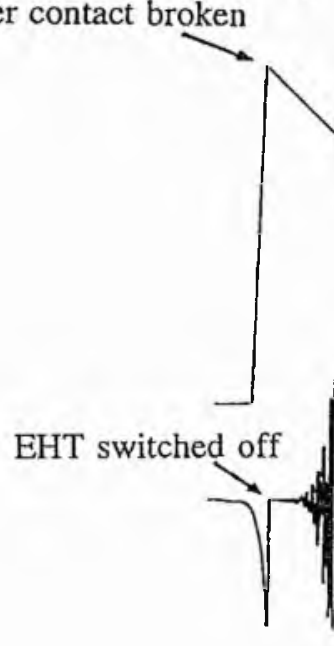

Increasing tensile force applied

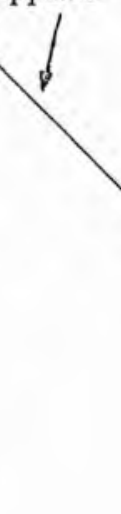

Compressive force offse reduced to zero

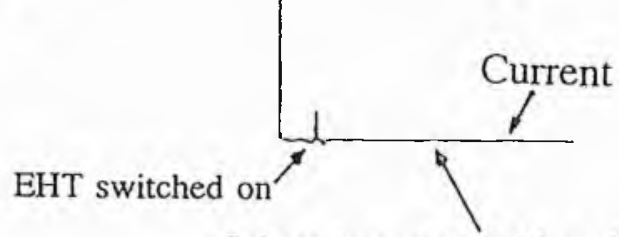

Sphere in contact with wall under small compressive force offset

Fig. 5.9 Chart recorder traces for electric current and force in detachment experiment. 


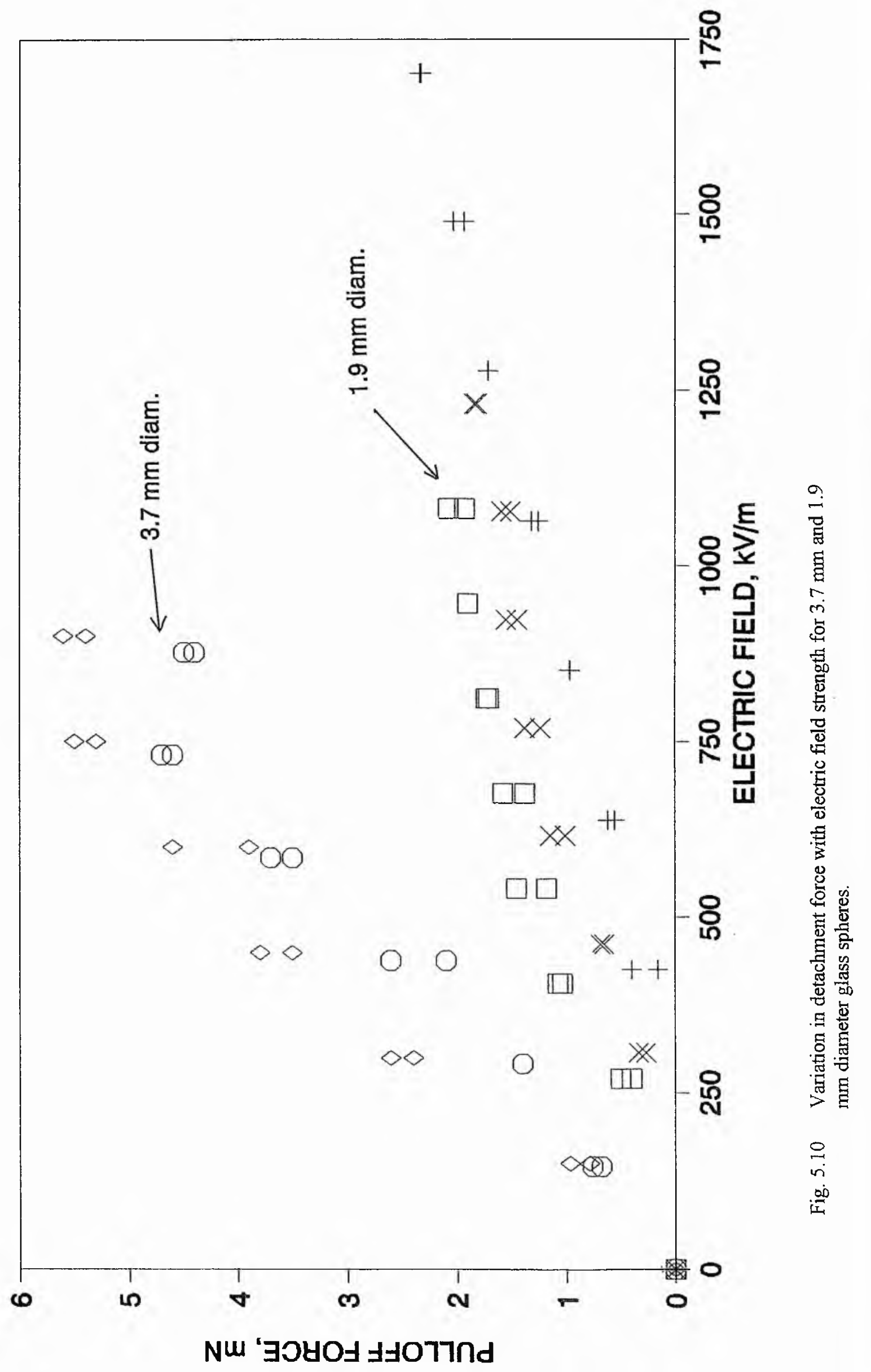




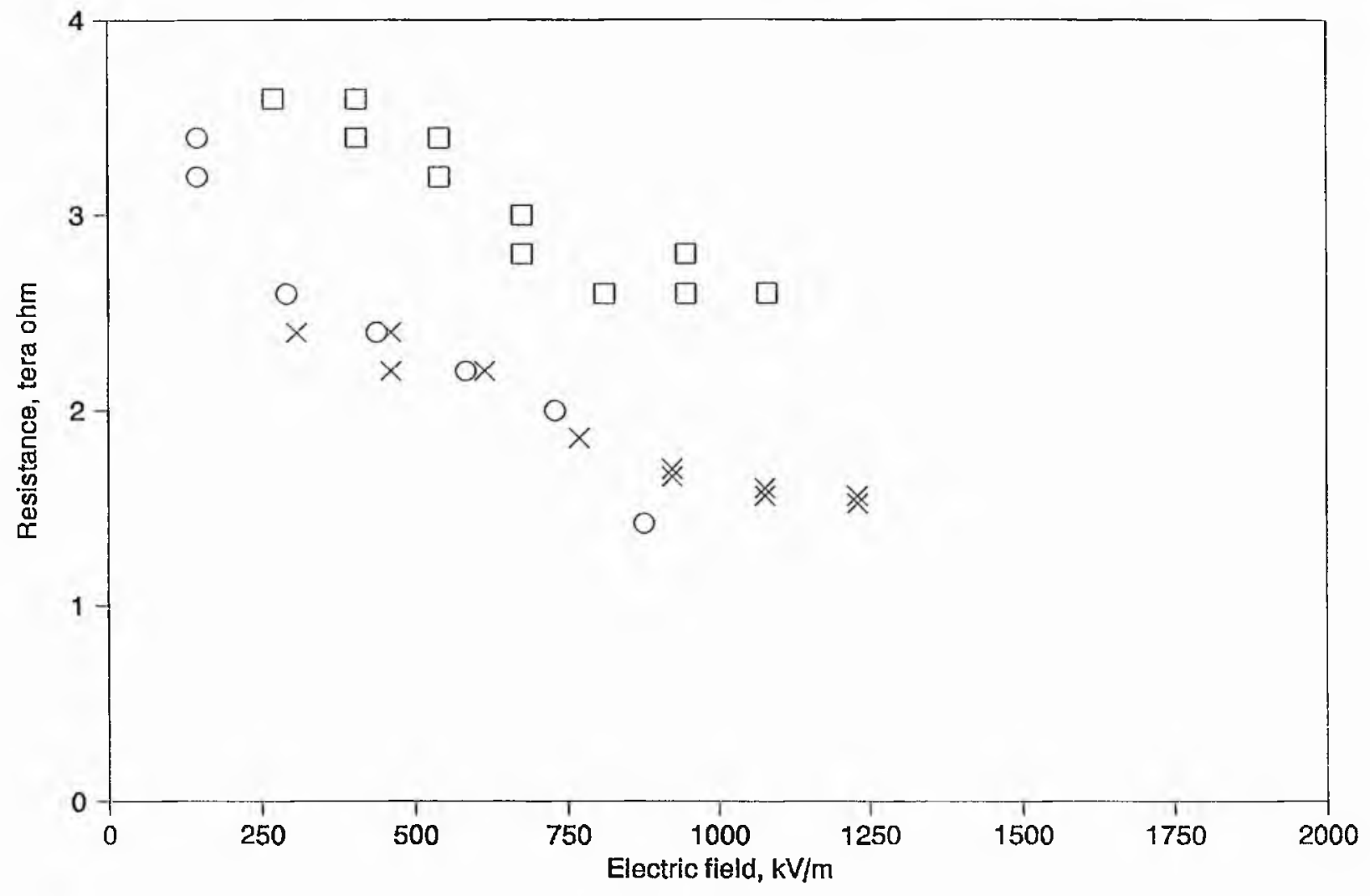

(a)

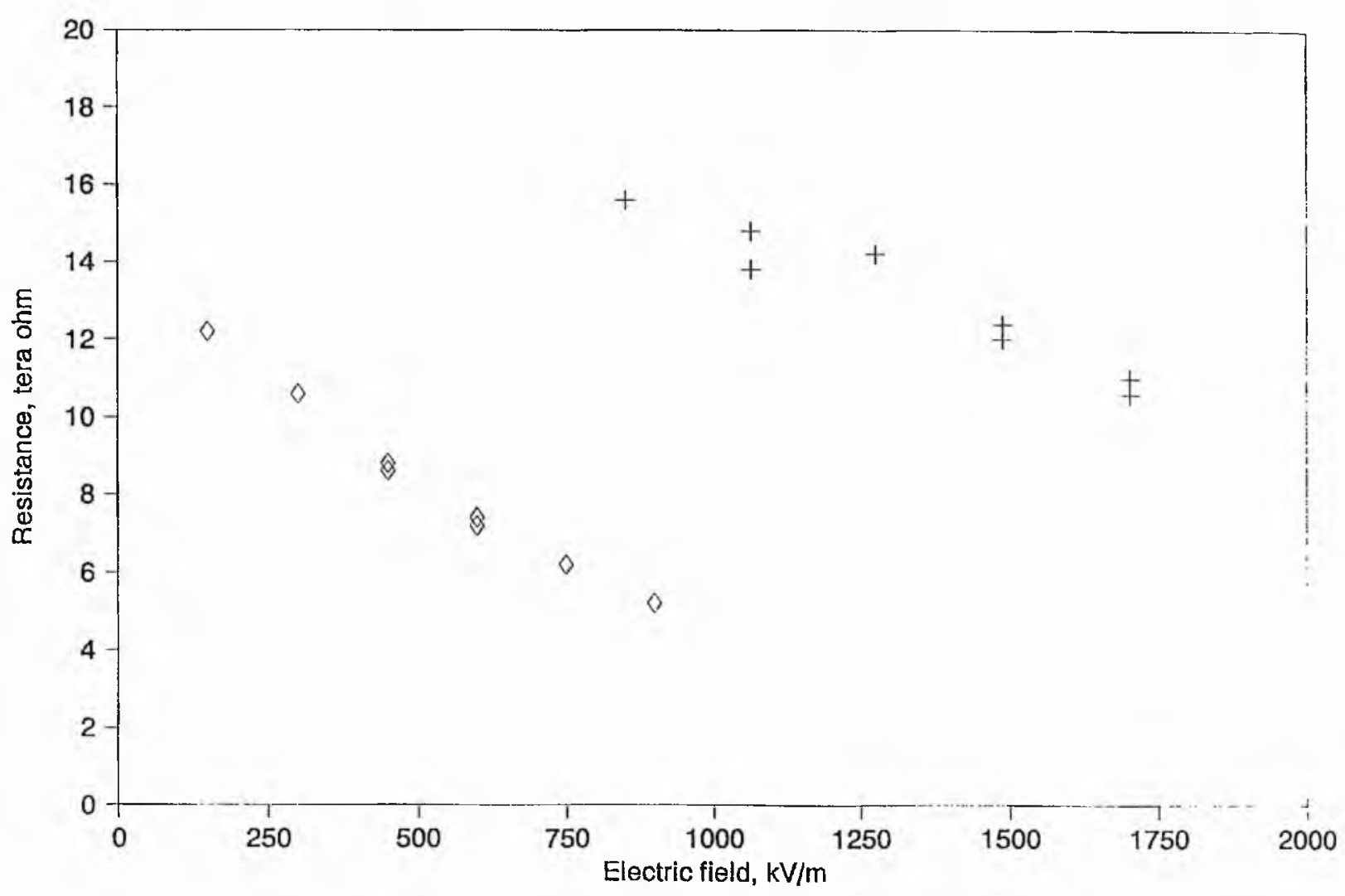

(b)

Fig. 5.11 Variation in resistance with electric field strength for glass spheres. Symbols correspond to same spheres in Fig. 5.10. (a) $1.9 \mathrm{~mm}$ dian. spheres: $X, \square$; $3.7 \mathrm{~mm}$ diam. sphere: $O$. (b) $1.9 \mathrm{~mm}$ diam. sphere: $+; 3.7 \mathrm{~mm}$ diam. sphere: 0 . 


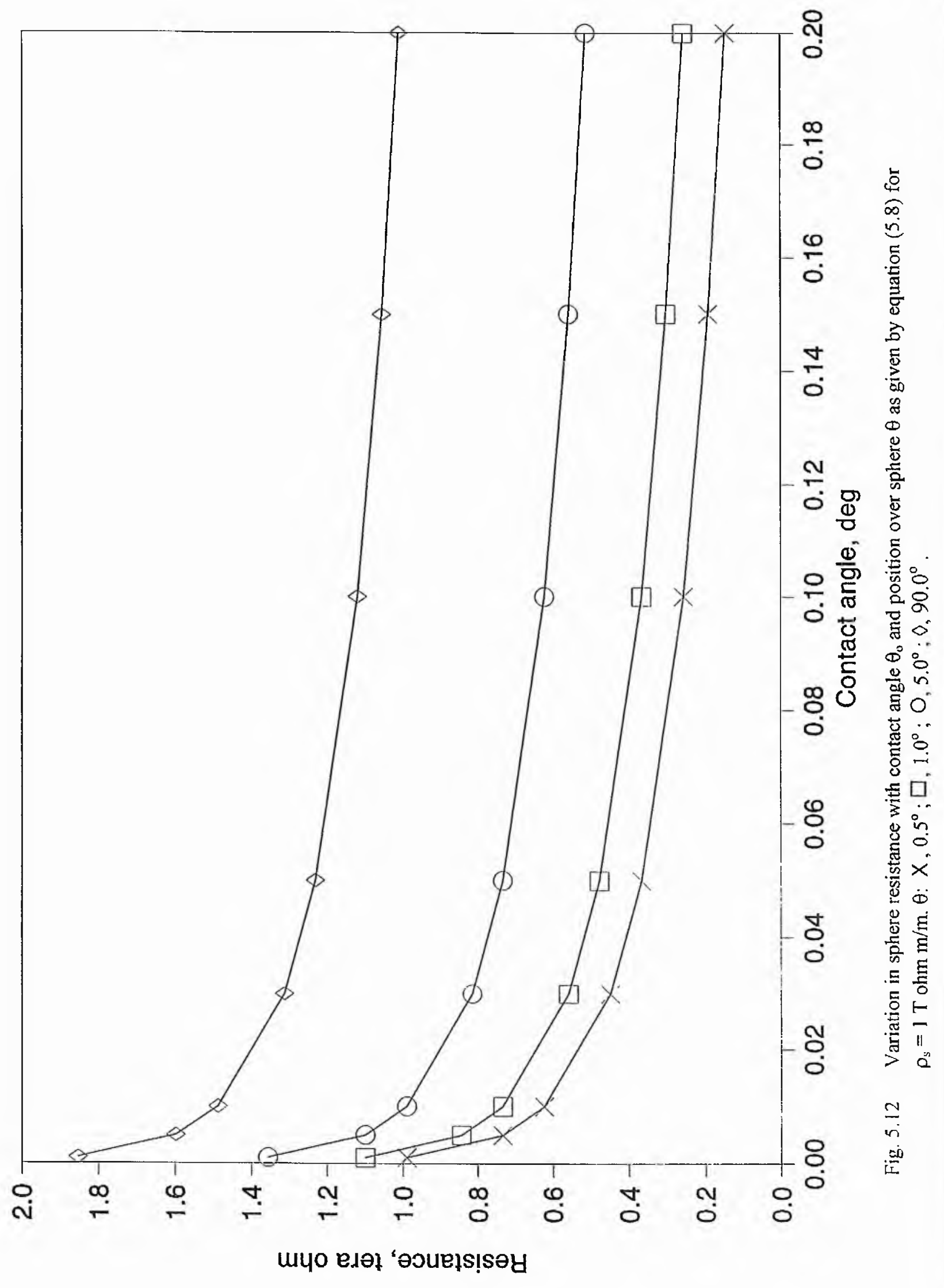




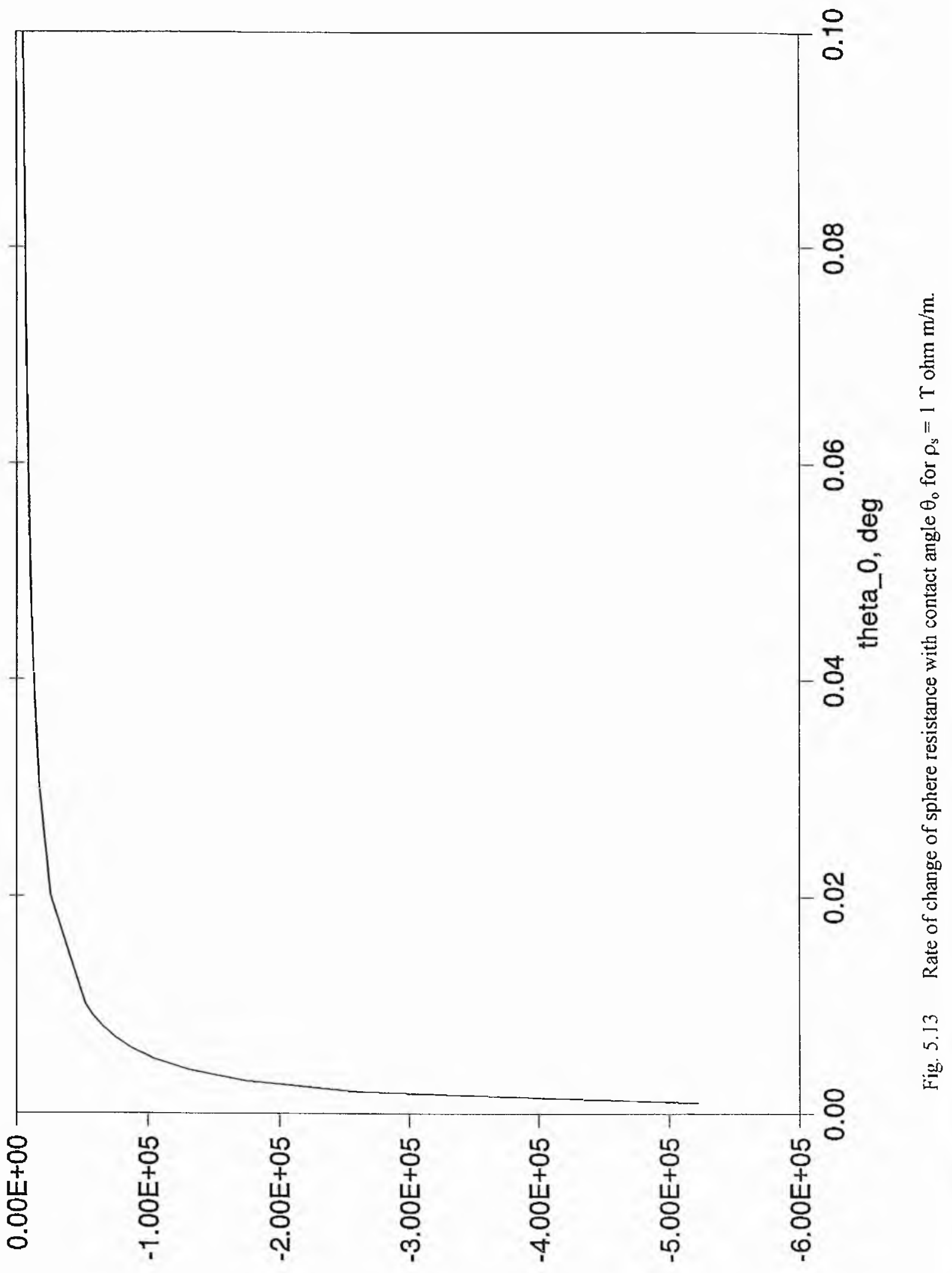

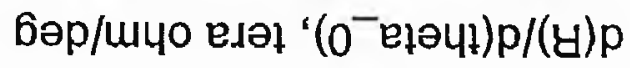




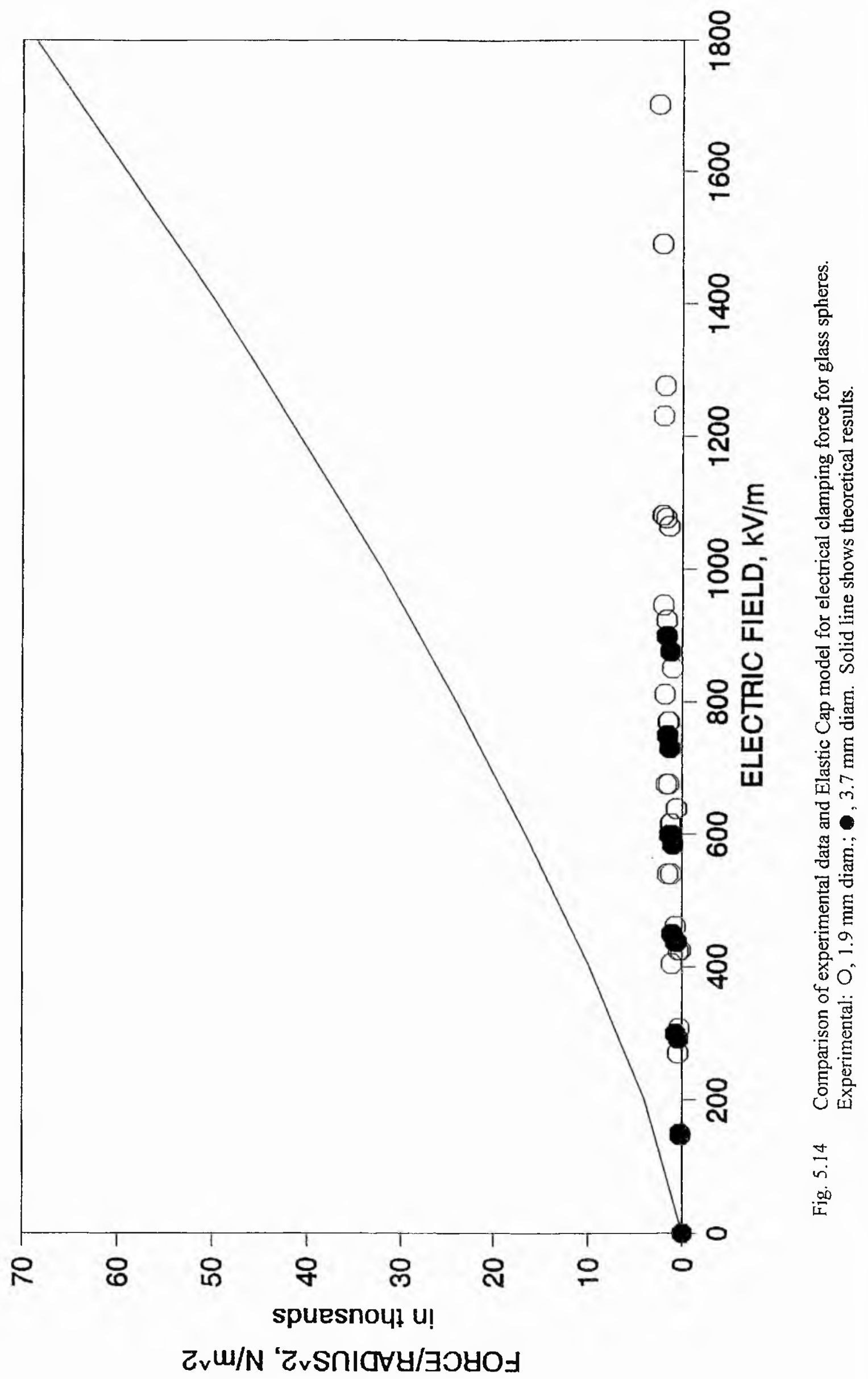




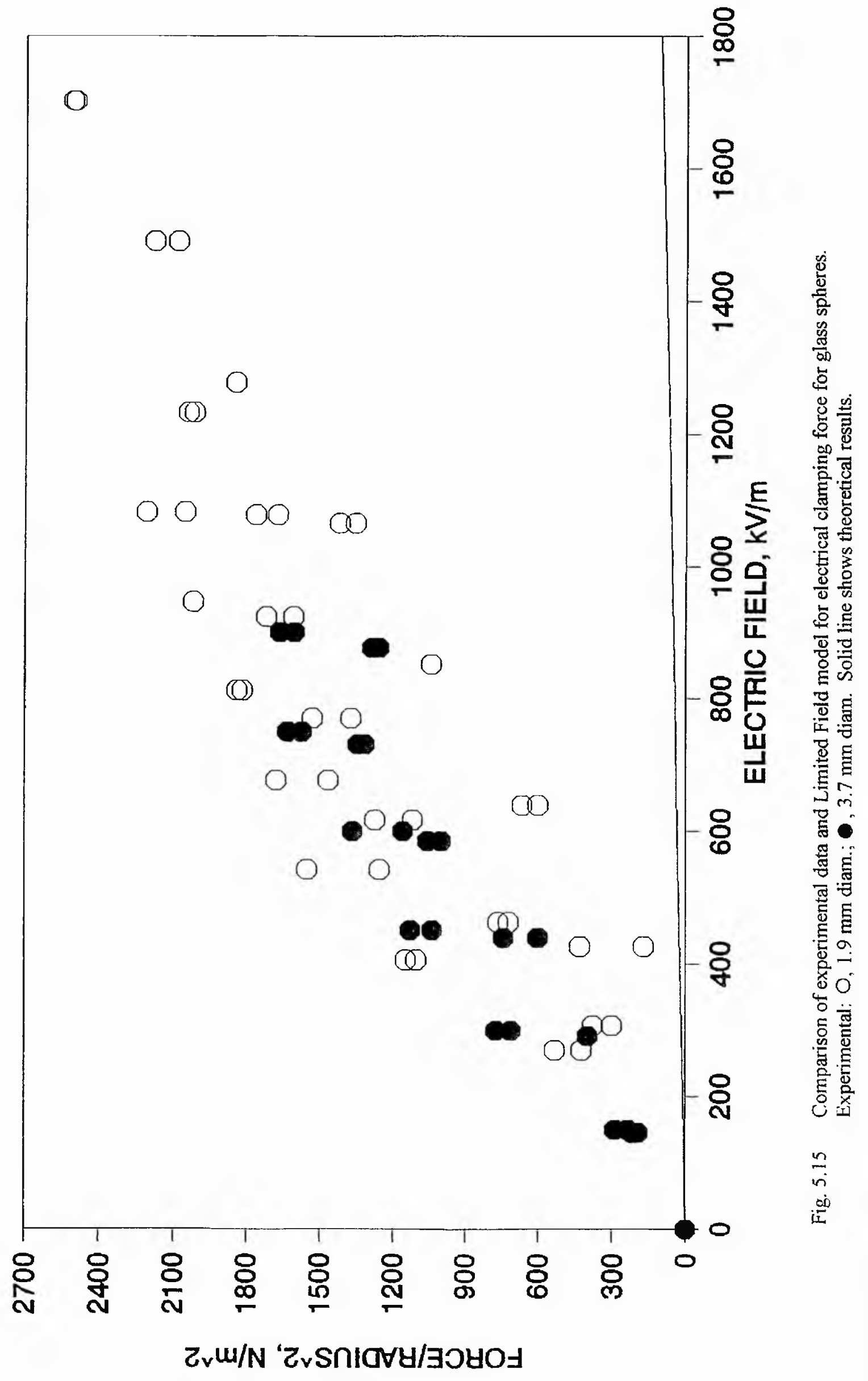




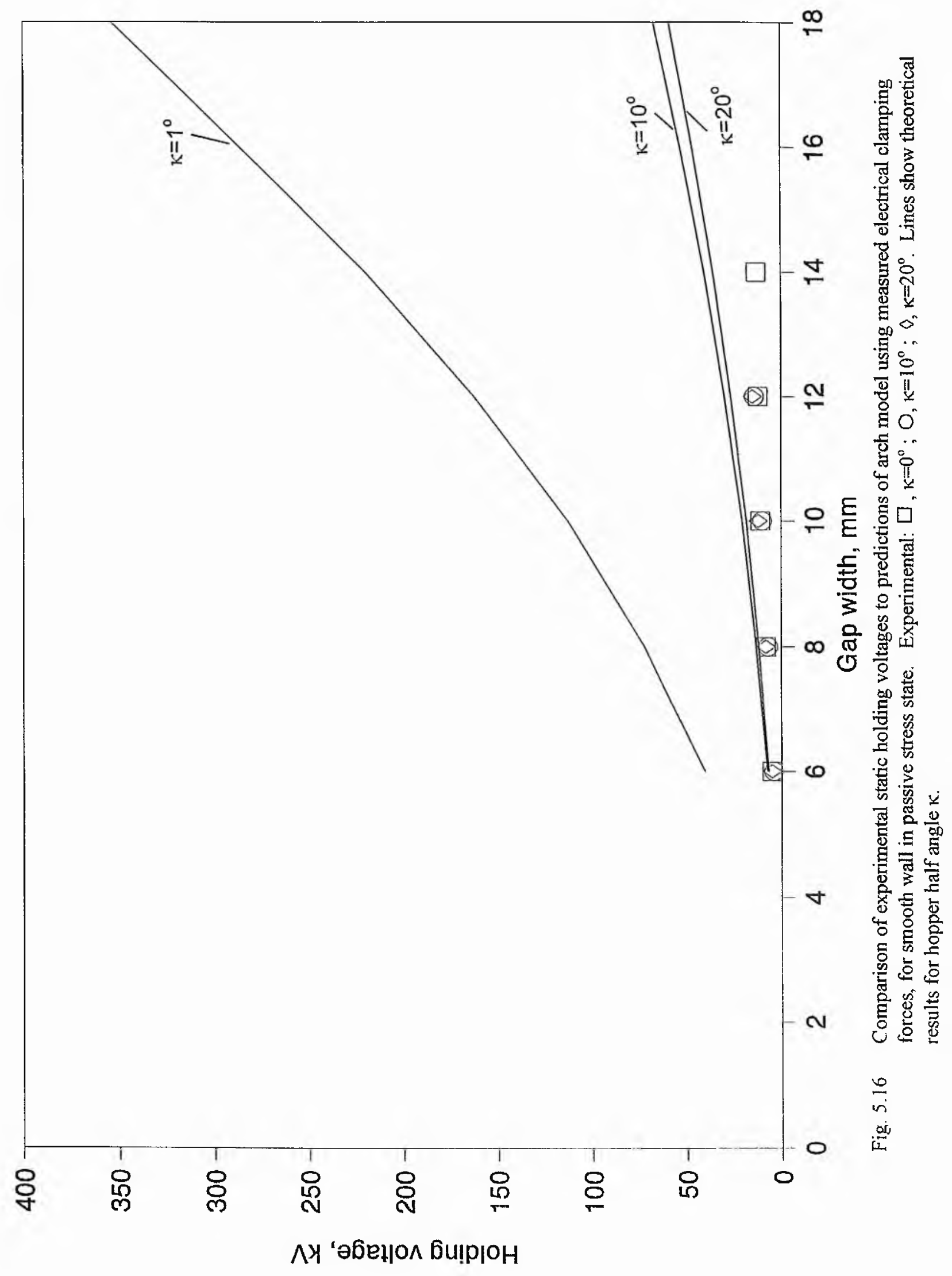




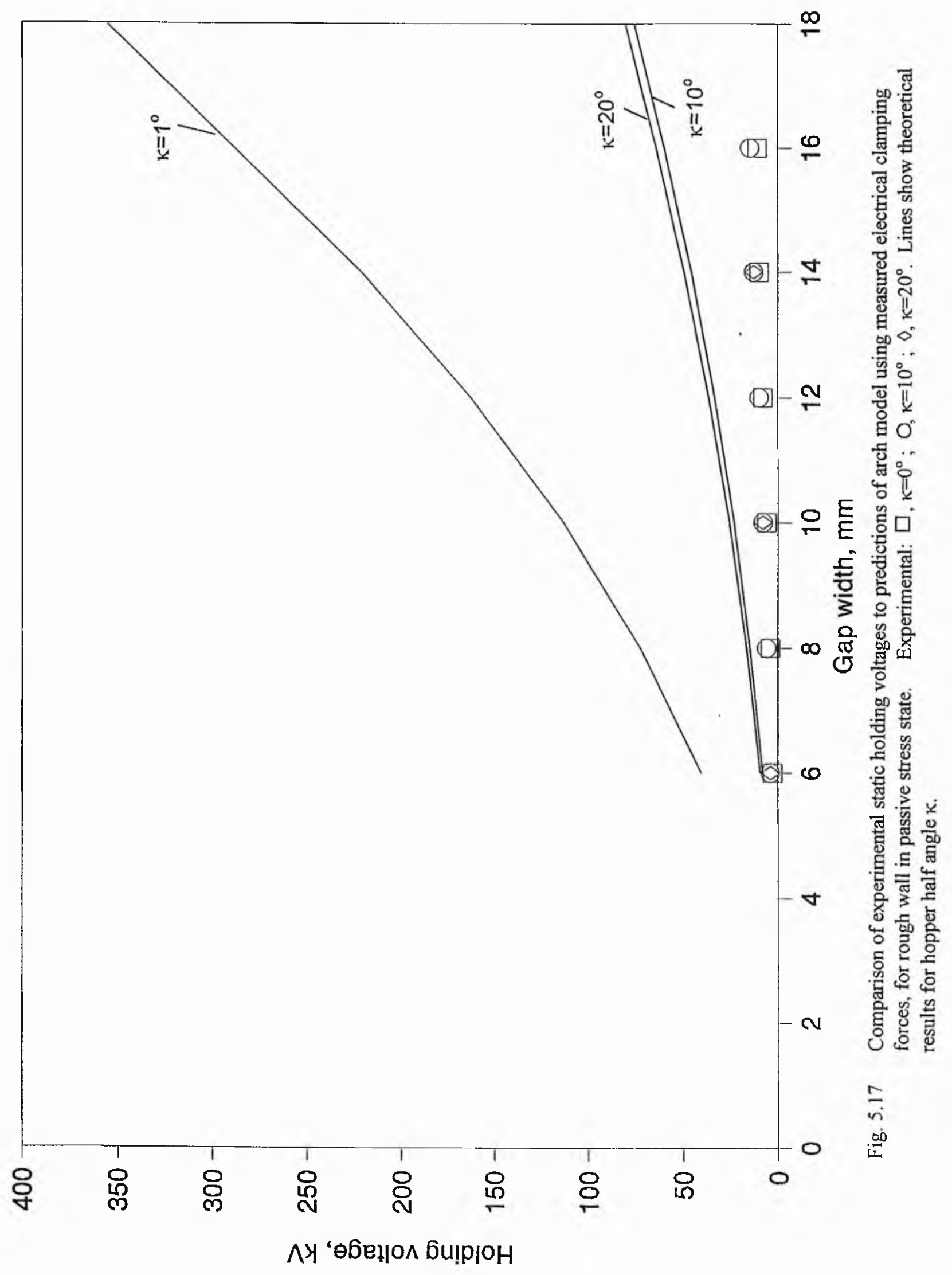




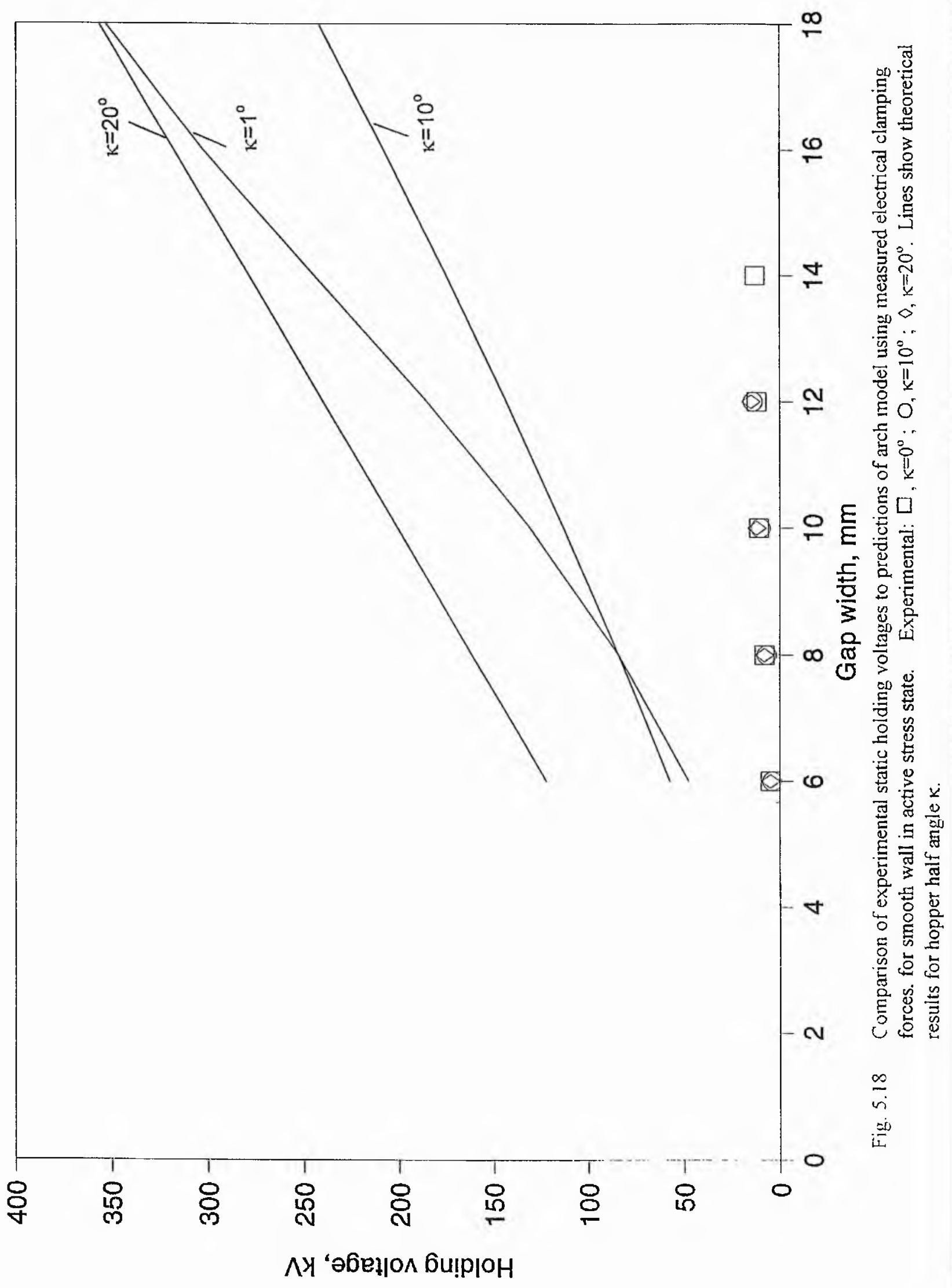




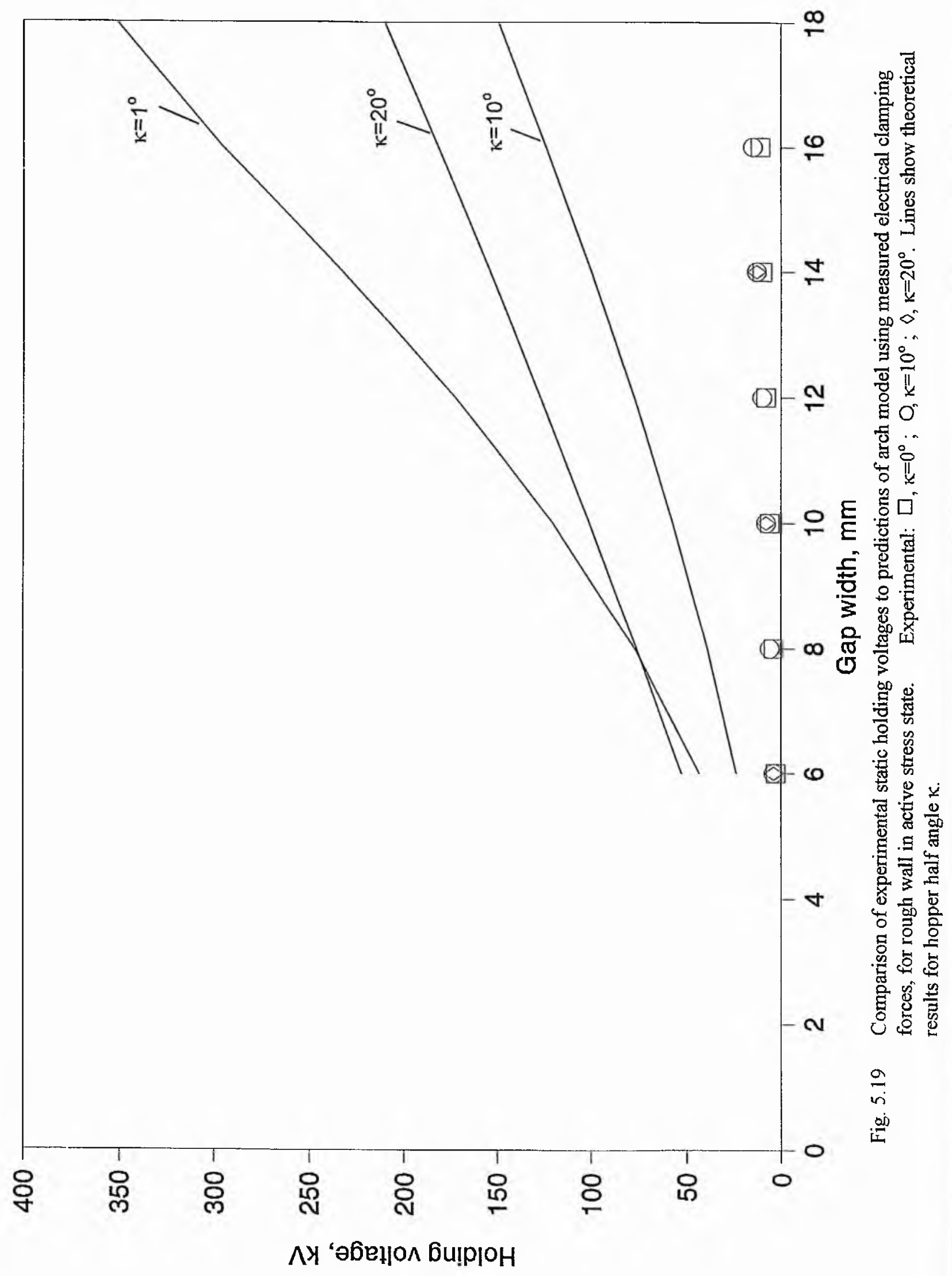




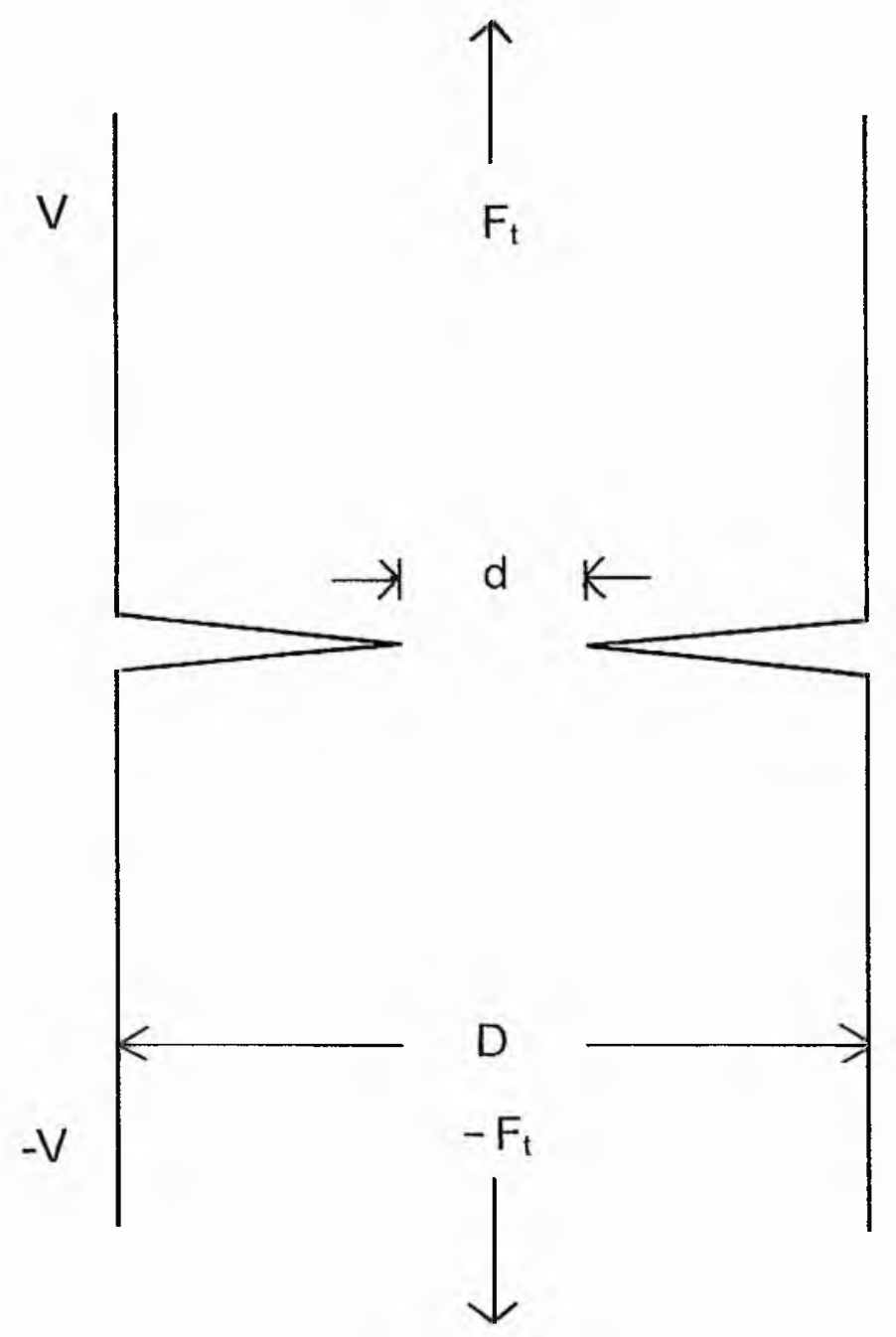

Fig. 5.20 Schematic diagram of deformation of axial contact between two cylinders when tensile force is applied in presence of electric field. 


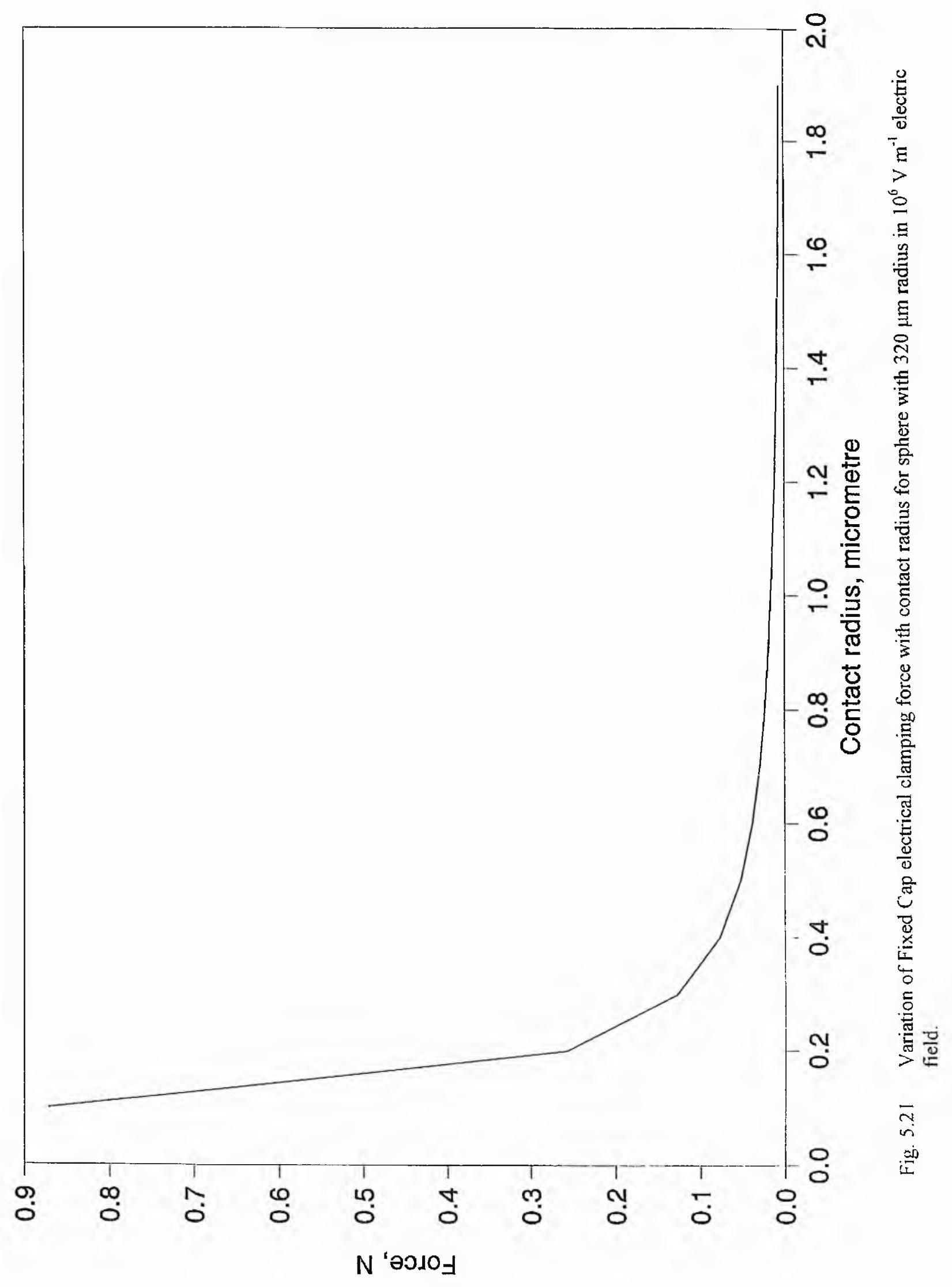




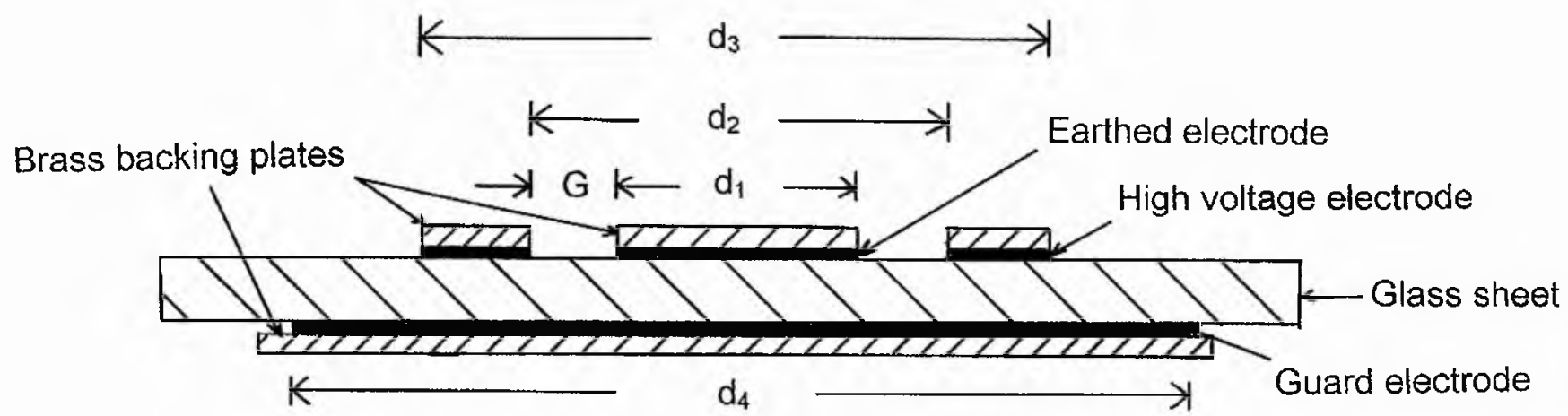

Fig. 5.22 Diagram of electrode setup for surface resistivity measurement. 


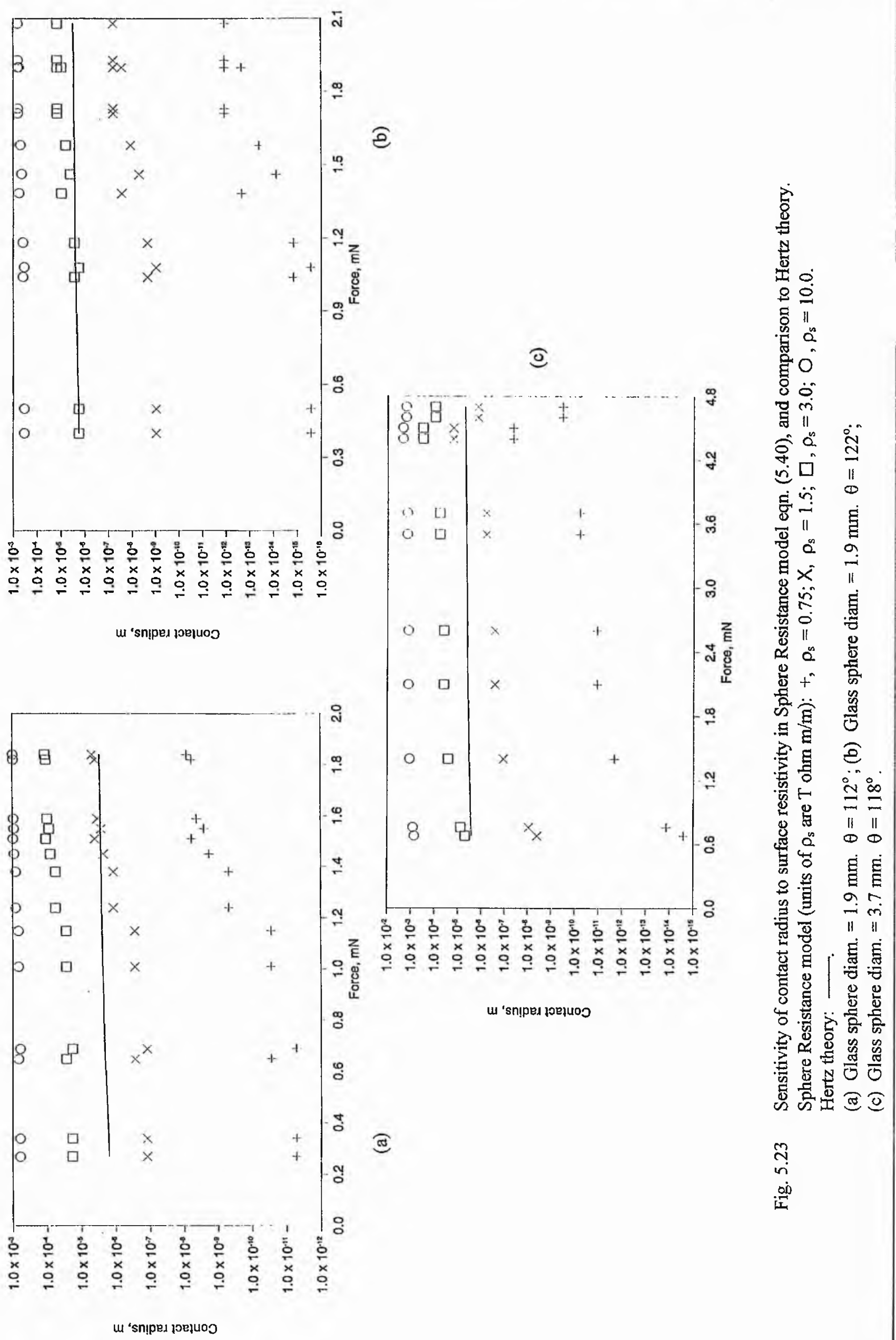




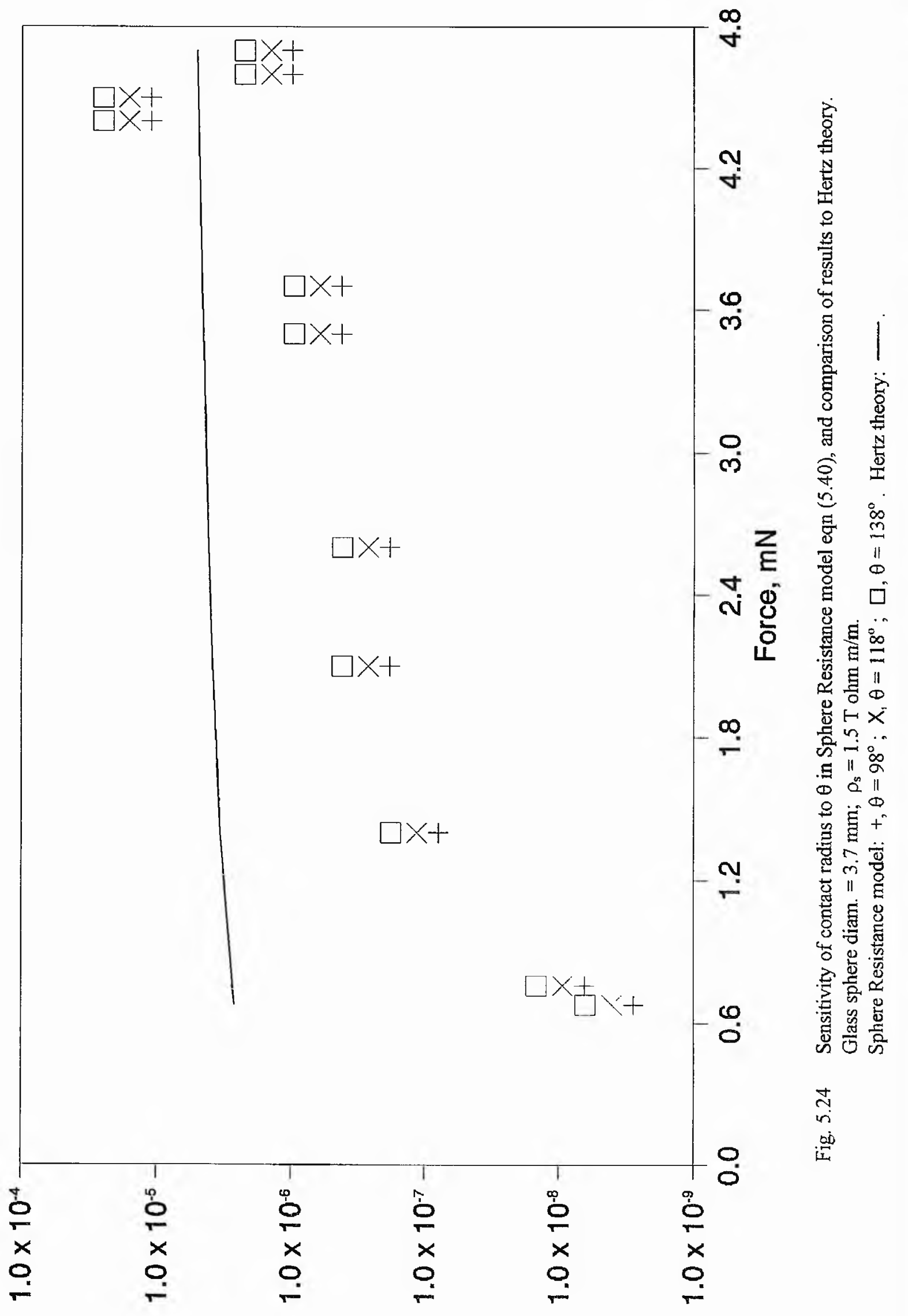

u ‘sn!ped łoełuoo 


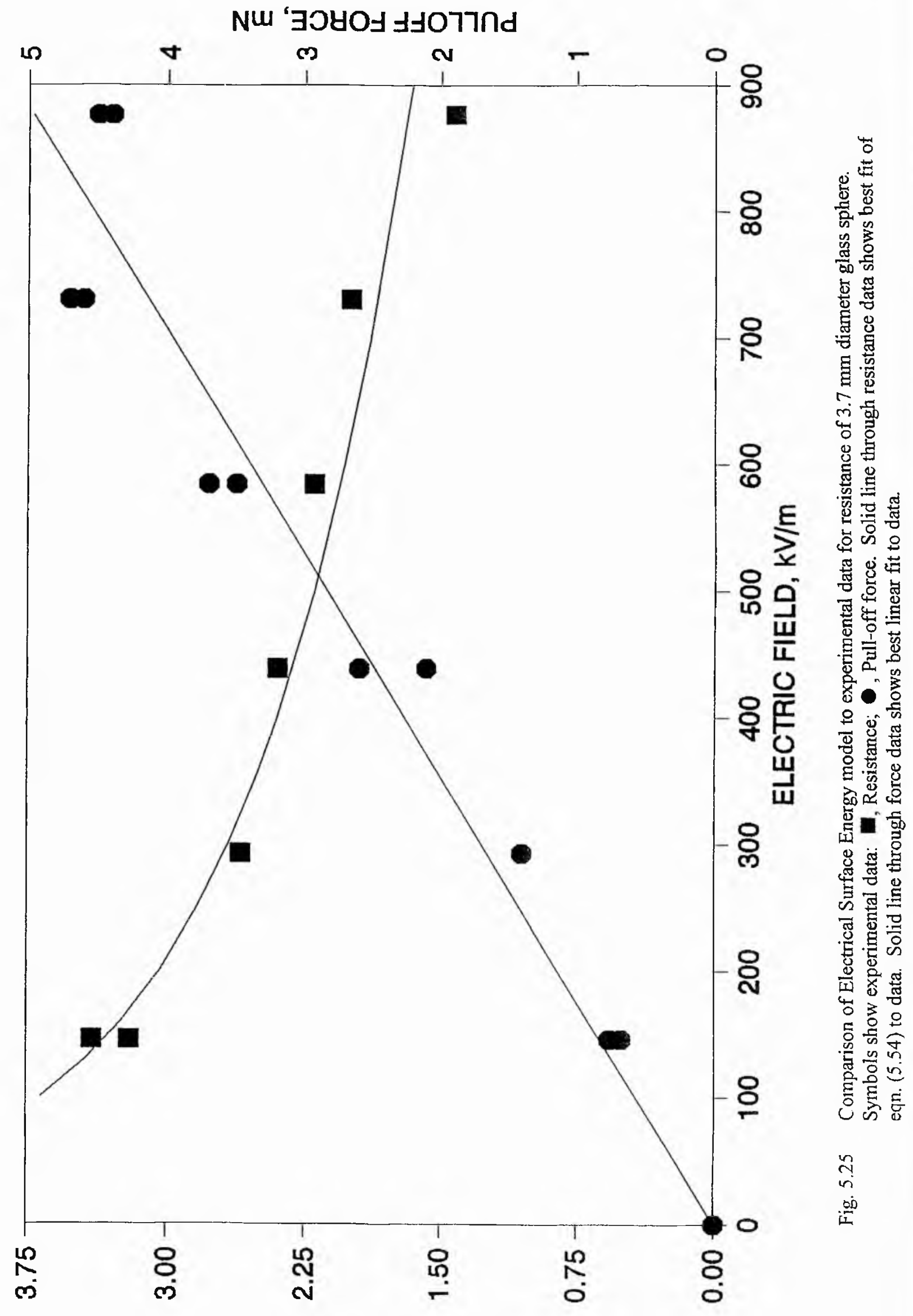

WHO $\forall Y \exists I$ ' $\exists O N \forall I S I S \exists H$.

HhuVh 